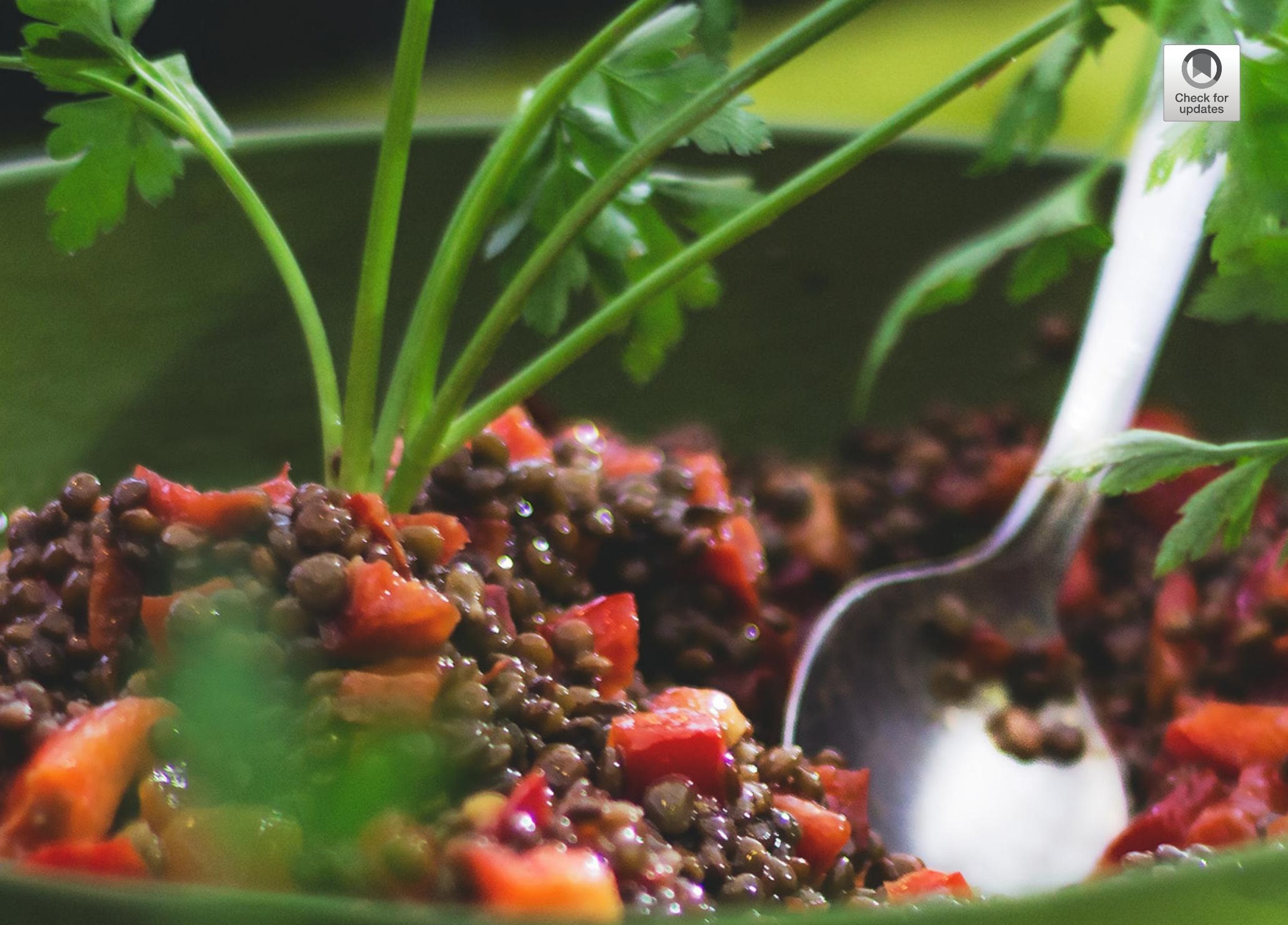

\title{
Phytoestrogens in foods on the Nordic market
}

A literature review on occurrence and levels

III) Nordic Council of Ministers 



\section{Phytoestrogens in foods on the Nordic market}

A literature review on occurrence and levels

Linus Carlsson Forslund and Hans Christer Andersson

TemaNord 2017:541 
Phytoestrogens in foods on the Nordic market

A literature review on occurrence and levels

Linus Carlsson Forslund and Hans Christer Andersson

ISBN 978-92-893-5046-4 (PRINT)

ISBN 978-92-893-5047-1 (PDF)

ISBN 978-92-893-5048-8 (EPUB)

http://dx.doi.org/10.6027/TN2017-541

TemaNord 2017:541

ISSN 0.908-6692

Standard: PDF/UA-1

ISO 14289-1

(C) Nordic Council of Ministers 2017

Cover photo: Unsplash.com

Print: Rosendahls

Printed in Denmark

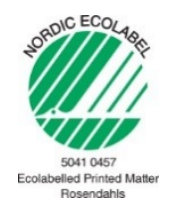

Although the Nordic Council of Ministers funded this publication, the contents do not necessarily reflect its views, policies or recommendations.

\section{Nordic co-operation}

Nordic co-operation is one of the world's most extensive forms of regional collaboration, involving Denmark, Finland, Iceland, Norway, Sweden, the Faroe Islands, Greenland, and Åland.

Nordic co-operation has firm traditions in politics, the economy, and culture. It plays an important role in European and international collaboration, and aims at creating a strong Nordic community in a strong Europe.

Nordic co-operation seeks to safeguard Nordic and regional interests and principles in the global community. Shared Nordic values help the region solidify its position as one of the world's most innovative and competitive. 


\section{Contents}

Preface

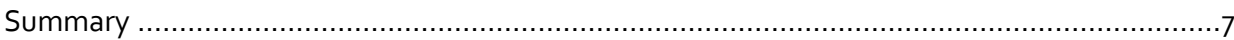

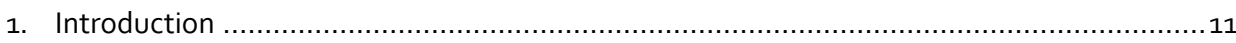

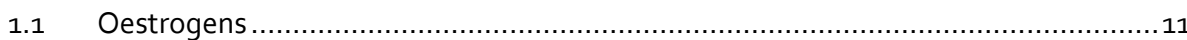

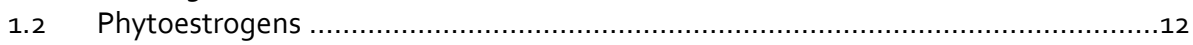

1.2.1 Identification of phytoestrogens ........................................................ 14

1.2.2 Relevant phytoestrogens in a food and feed context................................17

1.2.3 Food plants containing relevant phytoestrogens ................................19

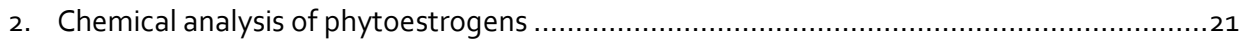

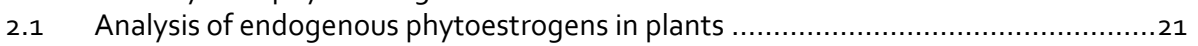

2.1.1 Extraction of isoflavones, lignans, and coumestrol .....................................21

2.1.2 Summary of analytical methods ................................................... 21

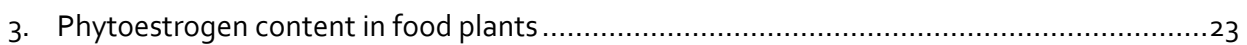

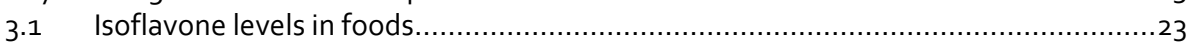

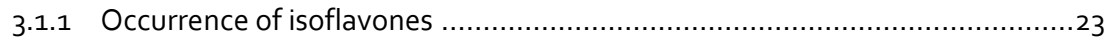

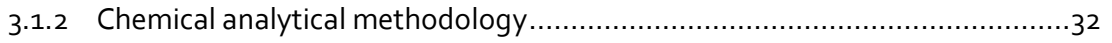

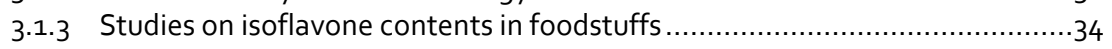

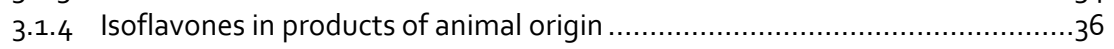

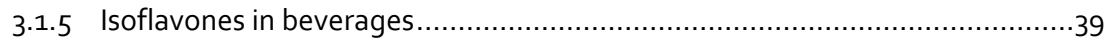

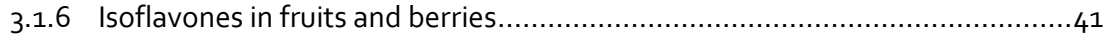

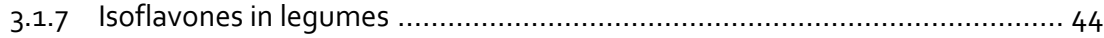

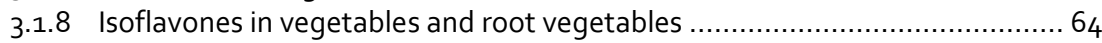

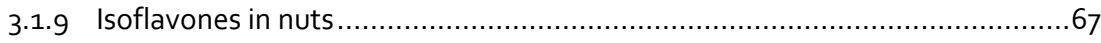

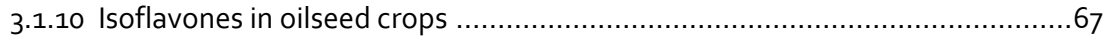

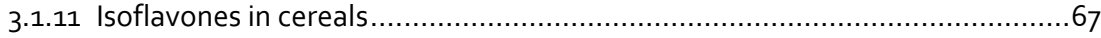

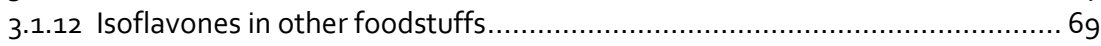

3.1.13 Isoflavones in dietary supplements ................................................... 73

3.1.14 Influence of storage and processing on isoflavone content........................74

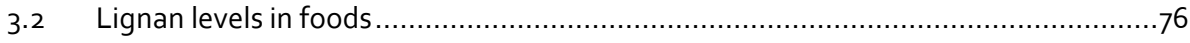

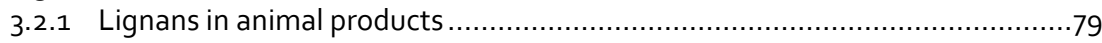

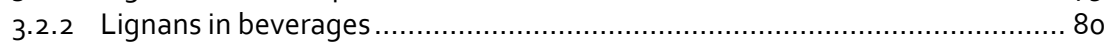

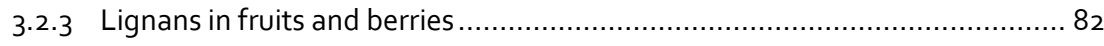

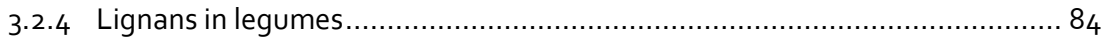

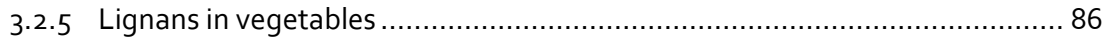

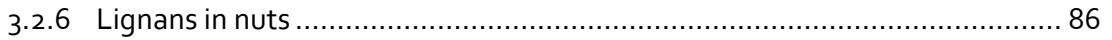

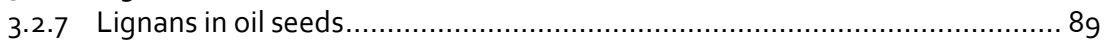

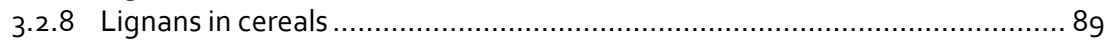

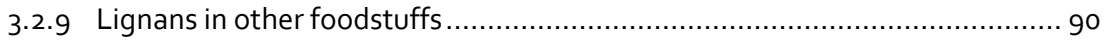

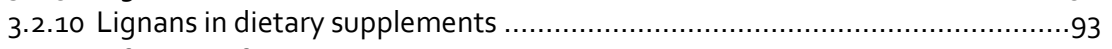

3.2.11 Influence of storage and processing on lignant content............................93 


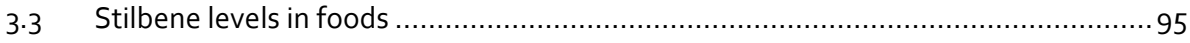

3.3.1 Stilbene production in plants........................................................... 95

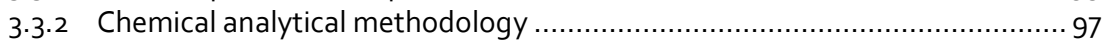

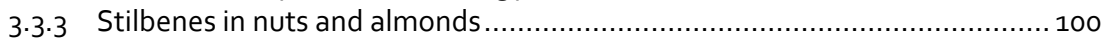

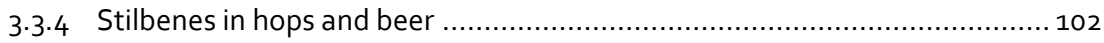

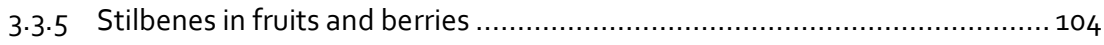

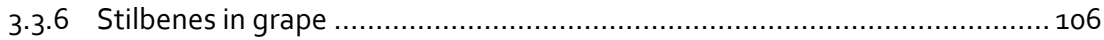

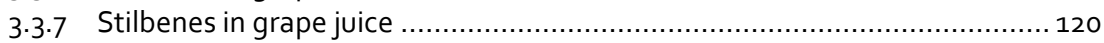

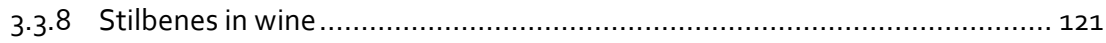

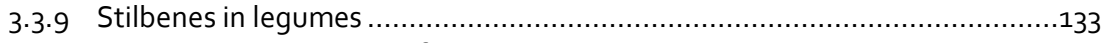

3.3.10 Stilbenes in solanaceous food plants ................................................133

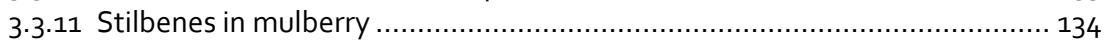

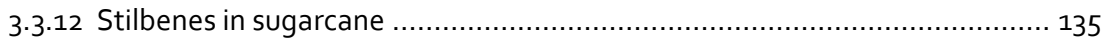

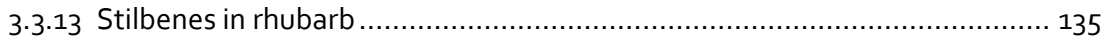

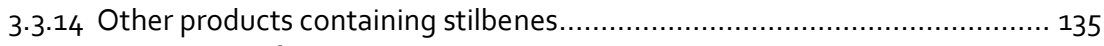

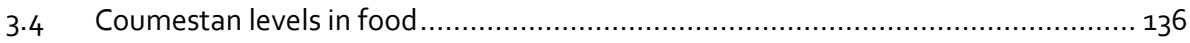

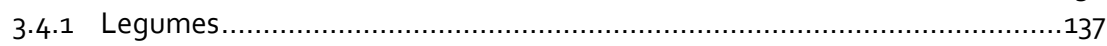

3.4.2 Occurrence in other foods than legumes .......................................... 139

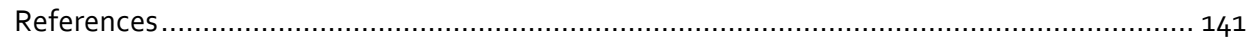

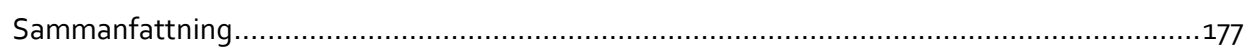




\section{Preface}

In many countries, there is a vivid discussion on compounds called endocrine disruptors. These compounds, assumed to interfere with the hormonal systems of humans and animals, are not well defined, partly because of the very large number of compounds that need to be considered. For example, they can be found among industrial chemicals and pharmaceuticals and are present in the environment, not least in our food.

The discussion on their occurrence in food has largely focused on environmental contaminants of the food, such as polycyclic aromatic hydrocarbons, bisphenol A, specific pesticides, dioxins, PCB and phthalates, but it should be considered that some oestrogenic compounds are common natural constituents of ordinary foods. Natural constituents of foods having a potential to mimic or interfere with the activity of oestrogens are usually called phytoestrogens. Microfungi-derived food contaminants with oestrogenic activity (mycoestrogens) may sometimes be found in foods but are usually not included among phytoestrogens.

As the name suggests, phytoestrogens derive from plants and have been demonstrated to have oestrogen activity in some systems. For example, they may bind to the human oestrogen receptor with affinities similar to or lower than that of $17 \beta$-estradiol. Much of our understanding of reproductive problems (oestrogenic effects, teratogenic effects and abortion) resulting from the occurrence of phytoestrogens in the diet comes from farm animals. In sheep, the problems have been linked to their consumption of clover, a plant containing different types of isoflavone (glycosides of formononetin, daidzein, genistein and biochanin A). The isoflavone conjugates are hydrolysed in the sheep rumen and the isoflavone aglycones released. The aglycones (formononetin and daidzein) are not intrinsically oestrogenic but are metabolised in the rumen to equol, which is the metabolite having oestrogenic activity. The compound is 0.00001 times as active as $17 \beta$-oestradiol.

Many phytoestrogens and endocrine disruptors, in general, have the potential to both mimic and mask effects of endogenous hormones. The masking could, for example, occur when the phytoestrogens are allowed to bind to the oestrogen receptor but are unable, possibly because of steric restrains, to promote activity. Therefore, it seems plausible that in some situations they may act as oestrogens; in other situations, they may block the activity of oestrogens. In this respect, the dose and the timing of the exposure are likely to be important. On top of this dual effect on the oestrogen receptors (ERs), many phytoestrogens also may have other types of biological effect. For example, they might inhibit particular enzymes or act as antioxidants, and antioxidants may under specific conditions be pro-oxidants!

Phytoestrogens have been suggested to ameliorate menopausal symptoms, which has stimulated their inclusion in health food products. However, a recent review 
gave no support for phytoestrogen pills reducing problems for post-menopausal women. It has also been claimed that long-term epidemiological studies suggest that women who consume a diet high in isoflavones may have a lower risk of endometrial and ovarian cancer. These partly controversial hypotheses have promoted the inclusion of phytoestrogen-containing products in foods.

To evaluate whether dietary exposure to phytooestrogens may result in negative effects or reduce the frequency of adverse effects, it is important to have good knowledge on the levels of the various phytooestrogens in normal foodstuffs. The present report aims at summarising the present knowledge on the levels of the following groups of phytoestrogens occurring in raw (unprocessed) foods: isoflavones, lignans, stilbenes and coumestans.

Factors that influence the level in the raw commodity have been critically assessed and the influence of food processing has been considered. There were no data available to allow a comparison to be done between levels in locally produced food (in the Nordic countries) and foods imported from other countries.

The work was performed by Linus Carlsson Forslund ${ }^{1}$ under the guidance of Hans Christer Andersson, National Food Agency, Sweden.

${ }^{1}$ Present address Thermo Fisher Scientific, Uppsala. 


\section{Summary}

For a long time, nutritional and toxicological aspects of the diet have focused intensely on primary metabolites. In the past three decades the discussion of potential beneficial and adverse effects of secondary metabolites in the diet has intensified. One class of compounds that has been under the spotlight in these discussions is the phytoestrogens, compounds that have the potential to act in a similar way to oestrogens in our bodies. The phytoestrogens may be active as such or after having been metabolised in our body. The active compound may bind to ER but with less affinity than the natural ligand $17 \beta$-estradiol. Food contaminants having oestrogenic activity and produced by microfungi are not called phytoestrogens but mycoestrogens. They have not been considered in the present report.

A reason for the blooming interest in phytoestrogens is the requirement to place the exposure of humans and animals to xenobiotic pollutants with estrogenic activity (such as plant protection product metabolites and bisphenol A) into perspective. Are the activities of the compounds introduced by man on the consumer qualitatively and quantitatively different from the activities of natural dietary constituents?

To evaluate whether dietary exposure to phytoestrogens may result in negative effects or possibly reduce the frequency of adverse effects, it is important to have accurate information on the levels of the various phytoestrogens in normal foodstuffs. The present report aims at summarising the present knowledge on the levels of phytoestrogens in raw (unprocessed) foods. Factors that influence the level in the raw plant commodity have been critically assessed and the influence of food processing has been considered.

It seems plausible that plants that have been domesticated and are used as food, like other plants, as an instrument of its own defence against feed searching animals and pests have developed ways to discourage them to be attacked. One such way could be to influence the signalling pathways in the attacker (e.g., its hormonal systems). To elucidate such a possibility, studies on the interaction between the compounds participating in the chemical defence and the attacking organisms are required, as are investigations into where the compounds can be found and what their levels are. The present report intents to contribute to the latter aspect and focuses on the occurrence of phytoestrogens in foodstuffs.

Information on the content of phytoestrogens in foodstuffs was collected from the literature identified in database searches in SciFinder and, when appropriate, in PubMed up to June 2014. The search terms used in these searches were phytoestrogen, the chemical group names (isoflavones, lignans, stilbenes and coumestan), the names of the individual compounds and search terms for different foodstuffs. Additional references were identified from the reference lists of the 
identified literature, except for coumestans for which there was no time to perform this task.

The identified literature was screened for data on phytoestrogens, and when available, was assessed for its quality. Data from poor studies were discarded. Because some investigators presented the data on a fresh weight ( $\mathrm{fw}$ ) basis and others on a dry weight ( $\mathrm{dw}$ ) basis and because the various investigators used different chemical methodologies to isolate and quantify the compounds, it became necessary in the presentation of the data to sometimes give data both on a fw basis and on a dw basis, as well as to separate data obtained with methods that could or was expected to hydrolyse glucosides from data obtained with methods intended to keep all compounds intact.

The present report summarises the present knowledge on the levels of the following groups of phytoestrogens occurring in raw, unprocessed foods: isoflavones, lignans, stilbenes and coumestans.

Isoflavones and coumestans have been analysed with very similar chemical analytical methods, but these methods have changed over time. The original studies focused on establishing the total content of isoflavones and coumestans and applied methods of extraction and clean-up that hydrolysed conjugated molecules and quantified the isoflavone aglycones daidzein, genistein, and glycitein, as well as the coumestan coumestrol. More recent methods attempting to identify the different compounds actually occurring in the plant and food have analysed twelve isoflavones (and coumestrol), the three aglycones mentioned above, their glucosides, malonylglucosides, and acetylglucosides. These studies have revealed that in the plant it is the malonylglucosides and glucosides that predominate. Legumes are particularly rich in isoflavones and coumestans, but the levels vary between species. High amounts are found in soybean, clover (red and white), and alfalfa. Other foods containing fair amounts of isoflavones and coumestans include products with soybean ingredients, some types of bread, energy bars, and some berries.

Lignans are compounds occurring in plant parts such as leaves, stems, flowers, and seed coats. They occur as aglycones or glycosides, but have been quantified as the free aglycones. Foods rich in lignans include cereals and seeds, legumes, and some vegetables and fruits. The lignans most commonly analysed for are secoisolariciresinol, lariciresinol, matairesinol, syringaresinol, pinoresinol, and medioresinol. When ingested, some of these are metabolised by intestinal bacteria to the mammalian lignans enterodiol and enterolactone that have phytoestrogenic activity.

Stilbenes are best known for their occurrence in grapes and grape products, but they are also found in, for example, beer (from the hops) and mulberry. The chemical analysis of processed foods such as wine has identified the aglycone resveratrol, its glucoside piceid, and dimers (e.g., vinefrins). The products contain both trans-forms and cis-forms. Studies of healthy grapes have demonstrated that stilbenes mainly occur in the trans-forms, indicating that the cis-forms frequently are formed by isomerisation upon stress and during processing. The particularly toxic vinefrines are likely to be produced in the orchestrated defence of the plant. 
Factors that influence the level of the phytoestrogens in the raw commodity have been critically assessed and discussed. It was recognised that most phytoestrogens are phytoalexins. Thus, their production in the plants is induced when the plant is exposed to various forms of stress, which could be abiotic, such as climatic conditions, UV light, and ozone, or biotic (such as attacks by insects, pests and microfungi). Against this background, it is understandable that many measurements (of healthy plants) have found low levels of phytoestrogens, whereas a few samples (presumably from stressed plants) contain high amounts of the phytoestrogen. It is concluded that it is unlikely that only a few analytical samples of a particular foodstuff will fully describe the level generally occurring in the food. Food processing is likely to remove or degrade some of the phytoestrogens, but a proportion of thermally stable compounds are likely to remain.

There was no data available to allow a comparison to be done between levels in locally produced food (in the Nordic countries) and foods imported from nonNordic countries. 



\section{Introduction}

\subsection{Oestrogens}

Oestrogens ${ }^{2}$ are primary female sex hormones, compounds important for the menstrual and oestrous reproductive cycles. Both natural and synthetic oestrogens are known. The natural oestrogens occur in mammals and some insects. Those occurring in mammals are steroidal hormones.

In common with other steroid hormones, oestrogens readily diffuse cell membranes. Having entered cells passively, oestrogens bind to and activate the oestrogen receptors (ER), a dimeric nuclear protein. The oestrogen-ER complex binds to specific DNA sequences called hormone response elements and thereby activate the transcription of a set of target genes. In experimental systems over 80 genes have been shown to be activated. Because oestrogen enters all cells, its actions are dependent on the presence of the ER in the cell. The specificity of the oestrogenregulated response is obtained by ER being expressed in specific tissues only, including the ovary, uterus, and breast.

The most important oestrogens in women are estrone (E1), estradiol (E2), and estriol (E3). Of these, estradiol is the predominant oestrogen during reproductive years (between menarche and menopause), both in terms of absolute serum levels and in estrogenic activity. Estrone is the predominant circulating oestrogen (as measured by serum levels) during menopause and estriol the predominant one during pregnancy. ${ }^{3}$ Although estriol occurs at the highest level of these oestrogens, it has the weakest activity. Estradiol has the strongest activity, approximately 80 times stronger than that of estriol.

While oestrogens are present in both men and women, they are usually present at significantly higher levels in women of reproductive age. In females, oestrogens are produced primarily by the granulosa cells of the ovarian follicles and corpora lutea in the ovaris, and during pregnancy also by the placenta. Both cell types in the ovaries are required to supply all enzymes necessary for their biosynthesis. Oestrogens promote the development of female secondary sexual characteristics, such as breasts, but are also involved in the thickening of the endometrium and other aspects that regulate the menstrual cycle. Highest levels occur near the end of the follicular phase just before ovulation. Smaller amounts of some oestrogens may also be produced by other tissues, including the liver, adrenal glands, and the breasts. These secondary sources of oestrogens are especially important in post-menopausal women. In males,

\footnotetext{
${ }^{2}$ After the Greek words oistros and gen, which mean inspiration (sexual passion or desire) and producer of, respectively.
} 3 There is also another type of oestrogen called estetrol that is only produced during pregnancy. 
oestrogen regulates certain functions of the reproductive system important to the maturation of sperm and may be necessary for a healthy libido.

In addition to the natural oestrogens mentioned above, a range of synthetic and other natural substances (not necessarily steroids) have been identified to possess estrogenic activity, although usually not of comparable activity to estradiol. The synthetic non-steroidal substances are known as xenooestrogens, whereas plant- and fungal-produced compounds with oestrogenic activity are known as phytoestrogens and mycoesterogens, respectively. Mycoestrogens can be found in food plants after a fungal infection. The most studied mycoestrogen, zearalenone, can be formed by several field fungi (e.g., Fusarium graminearum, F. semitectum, F. culmorum F. equiseti, F. ceralis), which can infect cereal crops (EFSA, 2004). The situation in which fungal infections produce oestrogenic mycotoxins in food plants will not be reviewed. This report reviews the occurrence of phytoestrogens in foods on the Nordic market.

\subsection{Phytoestrogens}

Phytoestrogens have been defined as plant constituents that can trigger biological responses in vertebrates and that can change or mimic the activities of endogenous oestrogens, in particular $17 \beta$-estradiol, predominantly by binding to ERs (FSA, 2003). They can elicit weak oestrogenic or antioestrogenic effects in mammals and are of two types: flavonoids and non-flavonoids (Kuiper et al., 1998; Cos et al., 2003; FSA, 2003; Steinshamn et al., 2008). The flavonoids can be further sub-divided into isoflavones, coumestans, and prenyl flavonoids. Non-flavonoids are of two types: lignans and stilbenes. The various types of phytoestrogen discussed in the present report are depicted in Figure 1 together with $17 \beta$-estradiol.

Isoflavones are restricted in their occurrence to the plant family Leguminosae to which soybean and chickpea belong (King \& Young, 1999). Examples of isoflavones are daidzein, genistein, glycitein, biochanin $A$, and formononetin. Formononetin and biochanin $A$ are precursors to daidzein and genistein, respectively, which are the most commonly found isoflavones (Cos et al., 2003). Equol is a metabolite of daidzein with phytoestrogenic activity, but only one third of the human population is able to transform daidzein into equol (Slavin et al., 1998; Rowland et al., 2000). 
Figure 1: $17 \beta$-Estradiol and the types of phytoestrogen discussed in the present report: $(A) 17 \beta$ estradiol, (B) isoflavones, (C) matairesinol (representative for the lignans), (D) coumestrol, and (E) trans-resveratrol (representative for the stilbenes)

(A)<smiles>C[C@]12CC[C@@H]3c4ccc(O)cc4CC[C@H]3[C@H]1CC[C@H]2O</smiles>

(C)<smiles>COc1cc(CC2COC(=O)C2Cc2ccc(O)c(OC)c2)ccc1O</smiles>

(B)<smiles>O=c1c(-c2ccccc2)coc2ccccc12</smiles>

(D)<smiles>O=C1Oc2cc(O)ccc2C2Oc3cc(O)ccc3C12</smiles>

(E)<smiles>Oc1ccc(/C=C/c2cc(O)cc(O)c2)cc1</smiles>

The coumestan, coumestrol, is an isoflavonoid-derived phytoestrogen found in clover and alfalfa (Cos et al., 2003). It is the most commonly found coumestan in food (FSA, 2003).

Prenyl flavonoids are present in almost all plants and have low or insignificant oestrogenic effects (Dixon, 2004). A few examples of prenyl flavonoids are naringenin, 8-prenylnaringenin (hopein), 6-prenylnaringenin, isoxanthohumol, and xanthohumol.

Lignans constitute a group of dimeric phenylpropanoids, contain a 2,3dibenzylbutane structure, and are widely distributed in the plant kingdom (e.g., in cereals, fruits and vegetables (Oomah 2002, Cos et al., 2003, Dixon, 2004). Some examples of lignans are secoisolariciresinol (SECO), matairesinol (MAT), pinoresinol, 
syringaresinol, and lariciresinol. Some lignans can be metabolised into enterodiol and enterolactone by intestinal bacterial flora (Heinonen et al., 2001).

Stilbenes are phenylpropanoids with a 1,2-diphenylethylene backbone (Chong et al., 2009). Plant families where stilbenes have been found include Vitaceae, Pinaceae, Poaceae, and Fabaceae. Resveratrol is the most studied stilbene and is found in, for example, wine (Chong et al., 2009).

Phytoestrogens are often conjugated with sugar molecules in plants and are then called glycosides (Dixon, 2004). The free form is called aglycones and is the form most efficiently absorbed in the intestines (Setchell et al., 2002; Manach et al., 2004; Peterson et al., 2010).

Phytoestrogens share several features in common with estradiol, including a pair of hydroxyl groups separated by a similar distance and the presence of a phenolic ring, which is a prerequisite for binding to the oestrogen receptor (Miksicek, 1995; Metzger et al., 1995).

\subsubsection{Identification of phytoestrogens}

Both in vivo and in vitro methods have been used in identifying potential oestrogenic dietary compounds.

\section{Observational studies}

A way to detect potential oestrogenic compounds is via observational studies in the field. Plants having phytoestrogenic activity were originally identified in observational studies on farm animals, initially observing decreased fertility in sheep (Bennets et al., 1946). Sheeps grazing on subterranean clover in Western Australia were found to manifest mainly three symptoms: infertility, maternal dystocia, and prolapse of the inverted uterus. Phytoestrogens have also been implicated in other studies regarding decreased fertility in California quail and cow (Leopold et al., 1976; Kallela et al., 1984).

The breeding success of California quail is irregular and depends on the amount of winter rainfall, with dry years leading to low reproduction (Leopold et al., 1976). The decreased fertility was hypothesised to be due to a change in diet during dry years to plants containing higher levels of phytoestrogens. Various plants in their diet were analysed for active constituents and the most commonly found phytoestrogens were the isoflavones: biochanin A, genistein, daidzein, and formononetin, but also the coumestan coumestrol. In a feeding trial, quail were fed either a diet composed only of turkey starter diet ( $26 \%$ crude protein), a low energy/low protein diet ( $15 \%$ crude protein), or a diet composed of turkey starter diet combined with an extract of subterranean clover that contained biochanin A, genistein, and formononetin. Quails fed the combined diet, including clover extract, laid fewer eggs than quail fed only turkey starter diet, indicating an inhibitory effect on reproduction by the isoflavones in the clover extract (Leopold et al., 1976).

Cows that were fed silage prepared from red clover after-growth, and later timothy-cocksfoot silage, showed reproductive disturbances (Kallela et al., 1984). After an investigation into the content of the silages, it was found that both contained 
the phytoestrogens daidzein, genistein, formononetin, and biochanin A, although very small amounts were detected in the timothy-cocksfoot silage. The reproductive difficulties gradually disappeared after the cessation of feeding with the silages. The red clover silage was implicated as the cause of the reproductive disturbances because of the results from an uterotrophic assay in rats. The red clover silage caused a significant increase in uterine weight, whereas the timothy-cocksfoot silage did not (Kallela et al., 1984).

\section{Uterotrophic bioassay}

The uterotrophic bioassay is an in vivo screening test for compounds having oestrogenic effects (OECD, 2007). The measured oestrogenic effect parameter is uterine weight or uterotrophic response in ovariectomised adult female or immature non-ovariectomised rodents. The compound is administered either by subcutaneous injection or oral gavage once a day for three days. The dosing period can be extended to seven days for the ovariectomised adult rodents to detect weak oestrogenic compounds $(O E C D, 2007)$. Phytoestrogens are expected to increase uterine weight.

\section{One- and two-generation tests}

The one- and two-generation tests are two comprehensive tests designed to investigate the influence of compounds on the performance of the male and female reproduction system in vivo (OECD, 1983; OECD, 2001). These tests are generally not used to identify oestrogenic compounds, but primarily to identify their specific oestrogenic effects.

\section{Ligand-ER binding assays}

The ligand-ER binding assay measures the test substance's ability to displace estradiol (E2) from ER binding sites in vitro and is thus evaluating the substance affinity towards ER- $\alpha$ or ER- $\beta$ in relation to E2 (Kuiper et al., 1997; Saarinen et al. 2006). The quantification of the displacement can be done by using radio-labelled E2 or fluorescein-labelled E2 (Bolger et al., 1998).

\section{ER-promoter binding assays}

The in vitro ER-promoter binding assay measures the binding of ERs, liganded with the test substance, to oestrogen response elements (EREs) (Nikov et al., 2000; Saarinen et al., 2006). Quantification of the binding is simplified by using radiolabelled or fluorescein-labelled ERE (Saarinen et al., 2006).

\section{ER-co-activator binding assays}

Co-activators can help activate receptors to bind to promoters and increase gene expression. Depending on whether the test substance hinders or stimulates coactivator binding, it can have anti-oestrogenic or oestrogenic effects, respectively. Examples of such methods are described below.

The two-hybrid assay uses yeast cells as indicator organism to detect ER-coactivator binding in living cells (Nishikawa et al., 1999; Saarinen et al., 2006). In this in vitro assay, yeast cells are transfected with two plasmids carrying genes for the ER and 
the co-activator, each linked to a portion of the domain for yeast transcription factor GAL4 (Saarinen et al., 2006). If the co-activator (with the other portion of the GAL4) and the receptor interact with each other, in the presence or absence of an ER-ligand, GAL4 comes together and can activate the expression of the $\beta$-galactosidase reporter gene (Nishikawa et al., 1999; Saarinen et al., 2006).

Another method of this type is the glutathione S-transferase (GST) pull-down assay. GST and the ER are fused together to form a GST-ER fusion protein (Nishikawa et al., 1999; Routledge et al., 2000; Saarinen et al., 2006). This fusion protein is incubated with an ER-ligand (the test substance) and a radio-labelled co-activator. The formed protein complex is then purified and separated and then autoradiography is used to identify and quantify the binding of the GST-ER to the co-activator. The effectiveness of the ligand in eliciting a binding between the ER and the co-activator is then determined (Nishikawa et al., 1999; Routledge et al., 2000; Saarinen et al., 2006). An alternative to determining binding between the ER and the co-activator by using a radio-labelled co-activator is to use fluorescent-labelled ER (or ER-ligand) and coactivators that can be identified by fluorescence resonance energy transfer (FRET) (Zhov et al., 1998).

Transactivation assays and gene expression analysis

The transactivation assay is an in vitro assay that evaluates a compound's potential to activate ER-dependent transcription (Saarinen et al., 2006; OECD, 2012a). In the assay, cells without endogenous ERs are transfected with plasmids containing an ER and a reporter gene. The ER-ligand is then added and the potential transcription of the reporter gene is measured (Saarinen et al., 2006).

Ligand-induced ER-mediated gene activation can also be determined in cells with endogenous ERs by measuring mRNA and protein expression (Saarinen et al., 2006; OECD, 2012a).

\section{Cell proliferation assay}

Cells dependent on the presence of oestrogens for proliferation can be used to screen for oestrogenic and antioestrogenic compounds. The E-screen assay is such an assay, which makes it possible to assess the oestrogenic activity of chemicals by measuring cell proliferation (Soto et al., 1995). Cells are incubated with the specific chemical at different concentrations or in the presence (positive control) or absence (negative control) of E2. The oestrogenic activity is evaluated based on the chemical's relative proliferative potency (RPP), i.e. the ratio between the minimal concentration of E2 needed for maximal cell yield and the minimal concentration of the test substance needed to attain a similar effect. It is also based on the chemical's relative proliferative effect (RPE), i.e. 100 times the ratio between the highest cell yield achieved with the compound and E2 (Soto et al., 1995). 


\section{Biochemical assay}

Chemical compounds have the potential to cause a wide variety of oestrogenic effects. Some effects can be investigated by studying the protein activities in vitro using biochemical assays (Saarinen et al., 2006). One example is to study the differentiation of osteoclasts by measuring the activity and secretion of tartrateresistant acid phosphatase (TRAP) in nuclear factor kappa B-ligand (RANKL) stimulated ER- $\alpha$-transfected RAW264.7 cells (Kanno et al., 2004). Oestrogenic compounds like estradiol inhibit TRAP activity and the differentiation of osteoclasts (Katao et al., 2011).

\subsubsection{Relevant phytoestrogens in a food and feed context}

In vitro studies, utilising the transactivation assay, have shown that, out of the phytoestrogens tested, coumestrol, genistein, and zearalenone had the highest biological activity, followed by daidzein and glycitein (Kuiper et al., 1998; Morito et al., 2001; Mueller et al., 2004). The results from the in vitro studies on the potency of these compounds confirm earlier data obtained by uterotrophic bioassay. In this latter study, immature female mice (unknown strain) were administered the phytoestrogens coumestrol, genistein, daidzein, formononetin, and biochanin A via the diet for about four to six days. The mice were then euthanised and the uterine weights examined. The potencies were determined by the relative concentration (ratio between concentration of diethylstilbestrol (DES) and concentration of the tested phytoestrogen) needed to increase the uterine weight to $25 \mathrm{mg}$. In this uterotrophic assay, coumestrol had the highest relative potency, followed by genistein, daidzein, biochanin A, and formononetin. The lower activity of biochanin A and formononetin might partly be explained by a non-optimal conversion of these compounds to genistein and daidzein, respectively. Estrone, a human oestrogen secreted by the ovary, was also tested, showing a potency of almost 2000 times higher than coumestrol (Bickoff et al., 1962).

Farmakalidis and co-workers (1985) performed the uterotrophic assay with isoflavones in $\mathrm{B} 6 \mathrm{D}_{2} \mathrm{~F}$ mice. Weanling $\mathrm{B} 6 \mathrm{D}_{2} \mathrm{~F}$ mice were treated with phytoestrogens in four daily doses of $0.1 \mathrm{ml}$ for four days and euthanised for evaluation on day five (Farmakalidis et al., 1985). In the study, genistein (8 mg), genistin (12 mg), and daidzin (12 mg) were tested by administration of the compound by gastric intubation. Daidzein was not tested in this study. Genistein and genistin significantly increased the uterine weight of the mice. In a similar study, weanling CD-1 mice were gavaged daily with genistein (6 or $8 \mathrm{mg}$ ), genistin (12 mg), and daidzin (12 $\mathrm{mg}$ ) in four daily doses of $0.1 \mathrm{ml}$ for four days (Farmakalidis \& Murphy, 1984). When the uterine weights were analysed after euthanisation on the fifth day, only daidzin significantly increased the uterine weight of the mice after the values had been corrected for initial body weight (Farmakalidis \& Murphy, 1984). Song et al. (1999) performed similar studies with glycitein, demonstrating that the compound had stronger oestrogenic activity in the uterotrophic assay than genistein. In studies on the competitive binding abilities with $17 \beta-\left[\mathrm{H}^{3}\right]$ estradiol to the oestrogen receptor proteins of mouse uterine cytosol, 
diethylstilbestrol had $95 \%$ of the activity of $17 \beta$-estradiol, genistein $0.49 \%$, daidzein, $0.027 \%$, and glycitein $0.028 \%$.

In a similar study, Tinwell et al. (2000) administered coumestrol, $60 \mathrm{mg} / \mathrm{kg}$ bodyweight (bw), via oral gavage for three days to mature and immature ovariectomised and immature and intact Alpk:AP rats. All groups had significantly increased uterine weights compared with control animals.

In the OECD programme to validate the rat uterotrophic bioassay, several laboratories performed single-dose studies on genistein (Kanno et al., 2003a). Immature and intact female rats were either administered $300 \mathrm{mg} / \mathrm{kg}$ bw/day by oral gavage or $35 \mathrm{mg} / \mathrm{kg}$ bw/day via subcutaneous injection for three days. Young adult and ovariectomised female rats were administered genistein (35 mg/kg bw/day) via subcutaneous injection for three or seven days. Genistein significantly increased uterine weight in all tests.

Genistein was also tested in dose-response studies by numerous laboratories (Kanno et al., 2003b). Immature and intact female rats were either administered 20-500 $\mathrm{mg} / \mathrm{kg}$ bw/day by oral gavage or $1-80 \mathrm{mg} / \mathrm{kg}$ bw/day via subcutaneous injection for three days. Young adult and ovariectomised female rats were administered genistein (1-80 $\mathrm{mg} / \mathrm{kg}$ bw/day) via subcutaneous injection for three or seven days. Genistein was shown to produce significant dose-related increases in uterine weight. Two laboratories reported a significant increase in uterine weight at $20 \mathrm{mg} / \mathrm{kg}$ bw and two showed a significant increase at $60 \mathrm{mg} / \mathrm{kg}$ bw after oral administration. Most laboratories reported a significant effect at $15 \mathrm{mg} / \mathrm{kg}$ bw when genistein was administered by subcutaneous injection, regardless of whether the rats were ovariectomised. Also, Santell et al. (1977) reported oestrogenic effects of genistein in the uterotrophic assay performed on ovariectomised female Sprague-Dawley rats. When a diet delivering 150, 375, or $750 \mu \mathrm{g}$ genistein/g food was supplied to rats for five days, a statistically significant increase in uterine weight was noted at the two highest doses.

Jefferson and Newbold (2000) studied the effects of dietary phytoestrogens in mice (unknown strain) using the uterotrophic assay. Immature female mice were subcutaneously injected with different doses of either coumestrol, genistein, DES or E2 for three days. The doses of coumestrol and genistein needed to produce an effect on uterine weight comparable to DES and E2 were 1,000 (coumestrol) and 50,000 (genistein) times higher than these oestrogens. The higher potency of coumestrol than genistein observed in vivo was subsequently confirmed in a transactivation assay (Jefferson and Newbold, 2000).

It has recently been shown that matairesinol possess antioestrogenic activity and secoisolariciresinol can exert both oestrogenic and antioestrogenic effects (Tominaga et al., 2009; Abarzua et al., 2012). 7-hydroxymatairesinol was shown to be oestrogenic in a cell proliferation assay using MCF-7 cells (Cosentino et al., 2007). Isolariciresinol and pinoresinol were also shown to be antioestrogenic in a transactivation assay (Aehle et al., 2011). The mammalian lignans, enterolactone and enterodiol, which are formed by intestinal bacterial flora, have been shown to be antioestrogenic and oestrogenic in transactivation and ER-promoter binding assays (Aehle et al., 2011; Pianjing et al., 2011). Precursors to enterolactone and enterodiol have been identified 
as lariciresinol, matairesinol, 7-hydroxymataresinol, secoisolariciresinol, pinoresinol, and syringaresinol (Heinonen et al. 2001). Medioresinol is structurally similar to pinoresinol and syringaresinol and is assumed to be metabolised into enterolignans.

The most commonly reported phytoestrogens in food are the isoflavones: daidzein, genistein, glycitein, and the lignan secoisolariciresinol (Milder et al., 2005; Thompson et al., 2006; Kuhnle et al., 2007, 2008a, 2008b, 2009a, 2009b).

Coumestrol and genistein are two phytoestrogens with high binding affinity for ER- $\beta$ (Kuiper et al., 1998; Mueller et al., 2004).

The phytoestrogens, sesamin and sesamolin, are found in high levels of sesame seeds and sesame oils. However, they are primarily only found in these food products and will therefore be excluded in this report.

Considering the plant constituents showing relevant effects in assays for oestrogenic activity, it was considered appropriate to identify food plants used in reasonable amounts and containing the phytoestrogens in order to subsequently extract available data on the level of these compounds in the respective food plant.

\subsubsection{Food plants containing relevant phytoestrogens}

Phytoestrogens have been detected in a wide range of vegetables, legumes, berries, fruits, cereals, and nuts, as well as in processed food products. Typically, the phytoestrogen content is fairly low $(\mu \mathrm{g} / 100 \mathrm{~g})$ but can sometimes reach high levels ( $\mathrm{mg} / 100 \mathrm{~g})$. There are several Tables in Chapter 3 that supply information on the levels of the various phytoestrogens in foodstuffs.

The relevance of phytoestrogens for the consumer is related to their biological effects, which include, but is not limited to, oestrogenic and anti-oestrogenic activity. The biological effects of phytoestrogens are outside the scope of this publication. For information on the influence of health parameters the readers are referred to other reviews (e.g., Cassidy et al., 2000; Frémont, 2000; Cornwell et al., 2004; Jeandet et al., 2010). 



\section{Chemical analysis of phytoestrogens}

\subsection{Analysis of endogenous phytoestrogens in plants}

\subsubsection{Extraction of isoflavones, lignans, and coumestrol}

The methods for extracting isoflavones, lignans, and coumestrol from food products have varied between studies. The most common method has been to mix the food sample with a suitable solvent, followed by hydrolysis of glucosides to aglycones using enzymes or acidic or basic conditions, or using a combination of these. Using such methods, typically only the total aglycone content is measured and the original proportion of glycosides and aglycones remain unknown. During later years, more studies have tried to identify and quantify the individual compounds in the plant, and to do so, milder extraction methods are required.

For example, if extraction of isoflavones takes place at $80^{\circ} \mathrm{C}$ instead of at room temperature, the malonylated (and to some extent the acetylated) isoflavones are converted to the aglycones/glycoside forms. This has been confirmed by Aussenac et al. (1998), who reported that malonyl daidzin and malonyl genistin are the major constituents of the extracts at $20^{\circ} \mathrm{C}$, whereas the major components in extracts at $80^{\circ} \mathrm{C}$ are daidzin and genistin. A partial conversion to the aglycone forms was already observed at $60^{\circ} \mathrm{C}$. Thus, it seems clear that artefacts may occur under nonappropriate extraction conditions.

\subsubsection{Summary of analytical methods}

The analytical methods used in most studies are gas chromatography-mass spectrometry (GC-MS) and high-performance liquid chromatography (HPLC).

Gas chromatography-mass spectrometry (GC-MS)

This method has been used in many studies to analyse the phytoestrogen content in food. GC-MS has several work up steps in which a loss of phytoestrogens could occur (Wang et al., 2002a). Internal standards are therefore added to account for such losses, 4 but steps prior to the addition, i.e. extraction and hydrolysis, could have losses that cannot be accounted for and therefore the levels may be underestimated (Wang et al., 2002a). Another issue with GC-MS is that it is difficult to analyse phytoestrogens without derivatisation, which is due to their hydroxylated structures. 
Therefore, the results are only presented as total phytoestrogen content and not aglycone and glycoside content (FSA, 2003).

Liquid chromatography with mass spectrometry detection (LC-MS)

Using LC-MS avoids the need for derivatisation and it has about the same specificity as GC-MS (FSA, 2003). With LC-MS, it is possible to measure both aglycone and glycoside content when using aglycone and glycoside standards (FSA, 2003).

High-performance liquid chromatography (HPLC)

As with LC-MS, HPLC does not require derivatisation of phytoestrogens, but has less specificity than GC-MS (Wang, 2002a). The broad range in methods for sample preparation allows for analysis of both glycosides and aglycones of phytoestrogens. Detection methods that can be coupled to HPLC are UV, fluorescence, mass spectrometry, or electrochemical detection. Of these, the last three can improve the sensitivity of the analysis (Wang, 2002a). Quantification is done by comparing calibration curves from reference standards. However, there is a risk of overestimation of the levels if substances in the sample, but are not in the reference standard, coelute with other compounds during chromatography (FSA, 2003).

Capillary electrophoresis (CE)

CE has been used in a few studies and has a higher specificity than GC-MS and HPLC (Wang, 2002a). It can be used to analyse both glycoside and aglycone content. The detection methods that can be used are the same as for HPLC: UV, fluorescence, mass spectrometry, or electrochemical detection.

Other methods

A simple solid phase extraction of isoflavones from soy samples followed by matrixassisted laser desorption/ionisation time-of-flight mass spectrometry (MALDI-TOF MS) analysis can provide an isoflavone profile in two minutes and may serve as a tool to identify and study processing changes of isoflavones in soy products (Wang \& Sporns, 2000). However, the method has not yet gained popularity. 


\section{Phytoestrogen content in food plants}

\subsection{Isoflavone levels in foods}

Flavonoids are a subclass of phenolics, characterised by a chalcone $C_{6} C_{3} C_{6}$ structure that can be divided into the main six subclasses: flavonols, flavones, flavanones, flavan-3-ols, anthocyanidins, and isoflavones. Isoflavones have the general structure shown in Figure $1 \mathrm{~B}$. Isoflavones are one of several groups of stress-induced secondary metabolites produced by legumes and some other organisms. They are formed in the phenylpropanoid pathway (Dixon \& Paiva, 1995). The first enzyme leading into this pathway is phenylalanine ammonia-lyase (PAL), which converts phenylalanine to cinnamic acid. Via a series of hydroxylation, methylation, and dehydration reactions, different secondary metabolites are formed. An important step is the chalcone synthase (CHS)-catalysed condensation of one p-coumaroyl-coenzyme A molecule with three molecules of malonyl-CoA to produce a $C_{15}$ flavonoid skeleton. In legumes, isoflavone synthase (IFS) rearranges the flavonoid carbon skeleton, leading to the accumulation of a wide range of simple isoflavones, coumestans, pterocarpans, and isoflavans. In soybean, two types of isoflavone synthases, IFS 1 and IFS2, have been identified (Jung et al., 2000). Apparently, the promoters of these ifs1 and ifs2 genes respond differently to nodulation (Nod) and defence signals, at least in transgenic soybean roots (Subramanian et al., 2004). The ifs1 and ifs2 genes have been transferred into other plant species by genetic engineering, and it has been established that transformed tobacco, lettuce, tomato, petunia, and Arabidopsis thaliana can produce genistin/genistein (Jung et al., 2000; Yu et al., 2000; Liv et al., 2007; Shih et al., 2008; Franzmayr et al., 2012). These findings pave the way for engineering in the future of various food plants to produce compounds claimed to have health-promoting effects.

Isoflavones have been given a role as regulators of plant-microbe interaction and antioxidants. It is customary to divide compounds that help plants fight microbial disease into two groups: phytoanticipins, which are preformed, and phytoalexins, which are inducible. Isoflavones may function both as a phytoalexin and as a phytoanticipin.

\subsubsection{Occurrence of isoflavones}

Isoflavones have a restricted distribution in the plant kingdom and are preferentially found in the subfamily Papilionoideae of the Leguminosae. Their structural variation is surprisingly large and involves not only the number and complexity of substituents on 
the 3-phenylchroman framework but also different oxidation levels of the heterocycle and the presence of additional heterocyclic rings. In the excellent review on isoflavones by Dewick (1988), the author lists 629 aglycone structures. Not all of these occur naturally and only a few of them have been tested for having phytoestrogen activity. However, the number of isoflavone glycosides identified, such as genistin (genistein 7-O- $\beta$-D-glucopyranoside), is small in comparison with the large number of known flavonoid glycosides. O-glycosides predominate but a considerable number of C-glycosides have also been documented. A small number of 5-hydroxyisoflavonoid phytoalexins derived from genistein have been reported in the Leguminosae, the prenylated isoflavonone kievitone from Phaseolus vulgaris possibly being the most studied example.

The isoflavones can be found in some species of the Fabaceae, Leguminosae, or Papilionaceae, commonly known as the legume, pea, or bean family. This is a large and economically important family of flowering plants as it contains many important agricultural and food plants, including soybean (Glycine max), beans (Phaseolus), peas (Pisum sativum), chickpeas (Cicer arietinum), alfalfa (Medicago sativa), peanut (Arachis hypogaea), sweet pea (Lathyrus odoratus), carob (Ceratonia siliqua), and liquorice (Glycyrrhiza glabra). Some are well known weedy pests in different parts of the world: for example, broom (Cytisus scoparius), kudzu (Pueraria lobata), and Lupinus species.

Although scattered information is available on some rare isoflavones occurring in these food plants, the majority of data are limited to the isoflavones occurring in soybeans, some other beans, and peas, as well as in red and white clover. Table 1 presents the isoflavone aglycones and isoflavone conjugates for which there are enough data to conclude on their levels in foods or food plants. The aglycones in question are displayed in Figure 2. The rather skewed distribution of analytical data to these isoflavones instead of to other isoflavones is likely because they have been demonstrated to have phytoestrogen activity.

From the biosynthetic point of view, genistein is the simplest isoflavone occurring in Leguminosae. However, it is a central intermediate in the biosynthesis of more complex isoflavones. The phytoestrogen activity of genistein is related to the structural resemblance of genistein (and other isoflavones) to the potent natural oestrogen $17 \beta$-oestradiol. The appropriate distance between the $4^{\prime}$ - and 7 -hydroxyl groups of the molecule and the important phenolic ring confer ability to bind ER and sex hormone binding proteins. Therefore, genistein can exert both estrogenic and anti-estrogenic activity, the latter by competing for receptor binding with oestradiol. The potent oestrogen equol (a major metabolite of dietary isoflavones formed by the gastrointestinal flora) and genistein can displace bound oestrogen and testosterone from human sex steroid-binding protein. Thus, genistein and other phytoestrogens could potentially affect clearance rates of androgens and estrogens and therefore the availability of the hormones to target cells. It should be noted that genistein binds differently to human $\alpha$ and $\beta$ ERs (Barnes et al., 2000). Genistein shows a greater affinity to oestrogen receptor $\beta$ than to the classical oestrogen receptor $\alpha$. This difference in affinity might be of importance as the two receptor sub-types differ in their tissue distribution and possibly in biological activity. In vitro, on the other hand, 
genistein has higher efficacy in inducing production of a reporter protein through oestrogen receptor $\alpha$ than it has through receptor $\beta$, despite its higher affinity to oestrogen receptor $\beta$ (Barkhem et al., 1998). This situation should be considered when extrapolating the results of phytoestrogen administration experiments in animals to hormone-related diseases in humans.

Table 1: Commonly found isoflavones in food plants

Isoflavones Synonym

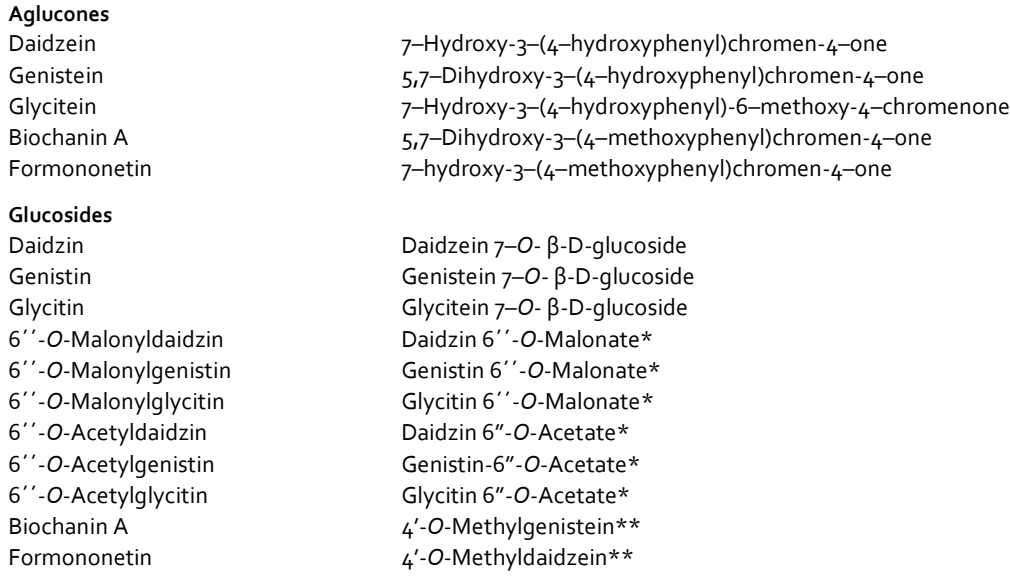

Note: *Isoflavone malonylated or acetylated at the 6"-position of the glucoside.

**Isoflavone methylated at the 4 '-position.

Figure 2: Isoflavone aglycons for which quantitative data in foods are available<smiles>[R]c1ccc(-c2coc3cc(O)c([R])c([R])c3c2=O)cc1</smiles>

$$
\begin{aligned}
& \text { Daidzein: } \mathrm{R}_{1}=\mathrm{H} ; \mathrm{R}_{2}=\mathrm{OH} ; \mathrm{R}_{3}=\mathrm{H} \\
& \text { Genistein: } \mathrm{R}_{1}=\mathrm{OH} ; \mathrm{R}_{2}=\mathrm{OH} ; \mathrm{R}_{3}=\mathrm{H} \\
& \text { Glycitein: } \mathrm{R}_{1}=\mathrm{H} ; \mathrm{R}_{2}=\mathrm{OH} ; \mathrm{R}_{3}=\mathrm{OCH}_{3} \\
& \text { Biochanin A: } \mathrm{R}_{1}=\mathrm{OH} ; \mathrm{R}_{2}=\mathrm{OCH}_{3} ; \mathrm{R}_{3}=\mathrm{H} \\
& \text { Formononetin: } \mathrm{R}_{1}=\mathrm{H} ; \mathrm{R}_{2}=\mathrm{OCH}_{3} ; \mathrm{R}_{3}=\mathrm{H}
\end{aligned}
$$

Isoflavones have been reported to perform several important physiological functions involved in the growth and development of legumes. For example, soybean isoflavones induce nod genes in Bradyrhizobium japonicum (Kosslak et al., 1987; Cho \& Harper, 1991a, 1991b, 1991c; Kape et al., 1991) and promote the formation of nitrogen-fixing nodules in the plant. Furthermore, they are associated with the response of soybeans to pests such as Phytophthora megasperma (Graham et al., 1990). Because of these physiologically important responses, it has been assumed that isoflavone content might be difficult to control by genetic means.

A few investigators have studied whether the level of isoflavone is the same in various compartments of the legume seed, or whether the level varies. When soybeans were cracked and separated into their anatomical parts (hull, hypocotyl, and cotyledon) by hand, the concentration of isoflavones was found to be highest in the 
hypocotyl $(14,000-17,500 \mu \mathrm{g} / \mathrm{g})$ and lowest in the hull of the seed $(100-200 \mu \mathrm{g} / \mathrm{g}$ ) (Eldridge \& Kwolek, 1983). Thus, about $1.5 \%$ of the hypocotyl are isoflavones. Cotyledons contained intermediate levels $(3,200-8,100 \mu \mathrm{g} / \mathrm{g})$, several times lower levels than in the hypocotyl. A similar distribution of total isoflavones in the seed compartments was reported by Kudou et al. (1991). De-hulling is expected to have negligible effects on total isoflavone content as the hull is very low in isoflavones (Eldridge \& Kwolek, 1983; Xu \& Chang, 2008b). The most common isoflavones in hypocotyl is daidzin and glycitin $7-\beta$-glucosides, whereas genistin is most common in the cotyledon (about 20 times as much as occur in the hypocotyl).

Romani and co-workers (2003) studied the isoflavone content in different parts of the soybean plant (cotyledons, stems, leaves, roots, and pods) at 21, 42, and 77 days after sowing. Total isoflavones in seed cotyledons were highest at the first sampling time (21 days). In the other tissues studied (stems, leaves, and roots), it increased with number of days after sowing. At the latest harvesting time (77 days), the highest amounts of isoflavones were found in the roots, with a great predominance of daidzein derivatives over genistein derivatives. The stem and leaves contained much lower quantities and only genistein derivatives. Pods were void of these compounds. In the dried beans, daidzein derivatives were more common than genistein derivatives, which were much more common than glycitein derivatives.

Ho et al. (2002) compared the flavonoid and isoflavonoid profile of soybean seeds and soy leaf, noting that the isoflavone profile of these two tissues were quite different. Soybean seeds were most abundant in malonyl-genistin followed by malonyl-daidzin, genistin, daidzin, genistein, and daidzein in decreasing order (in total, approximately $3500 \mathrm{mg} / \mathrm{kg}$ ). In contrast, soy leaves contained only trace amounts of malonyl-genistin and genistin (in total, $400 \mathrm{mg}$ isoflavones/ $\mathrm{kg}$ dry matter). No daidzein derivatives were found in leaves, at least in healthy plants. The structure of the malonylated and acetylated isoflavone glucosides are shown in Figure 3.

In a study performed in Illinois, USA, above-ground parts and flower heads of cultivated red clover were collected over one growing season and analysed for isoflavones (Booth et al., 2006). Daidzein and genistein contents peaked in June to July, whereas formononetin and biochanin A contents peaked in early September.

\section{Breeding for isoflavone content}

Several lines of evidence have indicated that it might not be straightforward for plant breeders to breed soybeans for high isoflavone content, in particular if the trait selection were combined with that of other traits (Meksem et al., 2001; Chiari et al., 2004; Gutierrez-Gonzalez et al., 2009).

Morrison et al. (2008) studied the influence of breeding and selection of soybeans over 70 years for yield on the isoflavone concentration of short-season cultivars. To do so, 14 historical cultivars released from 1934 to 1992 were grown at Ottawa for 12 years under identical cultural conditions. Seed samples, taken at harvest, were analysed for daidzein, genistein, and total isoflavones. Across the 58 years of breeding history, yield concentration increased by $0.43 \%$ and oil by $0.24 \%$ per year, whereas protein concentration decreased by $0.15 \%$ per year. Across the same period, daidzein, 
genistein, and total isoflavones increased by $1.04,1.47$, and $0.98 \%$ per year, respectively. It appeared as though recent cultivars with high isoflavone concentrations were more prone to environmental influence than older cultivars. Thus, selecting for higher yield in the short-season region could also imply indirectly selecting for higher isoflavone concentrations. The total isoflavone content in the studied cultivars varied between 1,630 and $2,984 \mathrm{mg} / \mathrm{kg}$.

Figure 3: General structure of malonylated and acetylated isoflavone glucosides

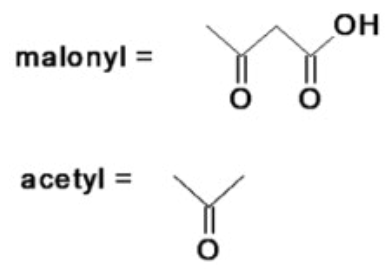<smiles>[R2]c1ccc(-c2coc3cc(OC4O[C@H](O)[C@H](O)[C@@H](O)[C@H]4O)c([R3])c([R1])c3c2=O)cc1</smiles>

Primomo et al. (2005) undertook a study to investigate whether breeding for increased isoflavone content would impact agronomic and other seed quality traits. It turned out that high and low isoflavone phenotypes were significantly different for maturity. In general, higher isoflavone content had a positive effect on agronomic traits. Isoflavone content had minimal effect on oil content, seed quality, and weight. There was a negative correlation to protein content but only in the population of low isoflavone content. However, also Chiari et al. (2004) reported a negative correlation between isoflavone and protein content in soybean seeds. Primomo and co-workers (2005) concluded that it is possible to develop soybeans with desirable isoflavone content in the seed and superior agronomic and seed quality traits.

\section{Factors influencing isoflavone content}

It was recognised early on that there is a large natural variation in the level of isoflavones in soybean as compared with the natural variation in other soybean constituents. A number of field trials have been performed in various soybean growing regions of the world to elucidate the factors responsible for the considerable natural variation in isoflavone content of soybeans. These studies have identified that both the genetic constitution of the soybean and the environment where the crop is grown have a major influence on the level of the different isoflavone constituents present in the harvested bean.

Isoflavones were extracted from the food matrix and analysed as described briefly in chapter 2. Some investigators hydrolysed the isoflavone conjugates during the extraction phase, using enzymes or acidic conditions, and were therefore only able to present data on total daidzein, total genistein, and total glycitein. Other investigators have extracted the isoflavone constituents under milder conditions and could identify and quantify the various conjugated and non-conjugated isoflavone forms. It should be noted that investigators that have not used validated methods of extraction might 
partly have hydrolysed the isoflavone conjugates in the preparation before the chromatographic step. As already pointed out in the previous chapter, an analysis of total isoflavones would be the case when constituents were identified and quantified by GC-MS.

In the following sub-sections, the various factors that have been claimed to influence the level of isoflavones in some foods are reviewed.

Genotype. There is an extensive database demonstrating that soybean varieties cultivated at the same place at the same time may contain very different levels of isoflavones. Apparently, the genetic constitution of the soybean is one of many factors that may influence the isoflavone content in the bean (Eldridge \& Kwolek, 1983; Wang \& Murphy, 1994a; Carrao-Panizzi \& Kitamura, 1995; Tsukamoto et al., 1995; Aussenac et al., 1998; Hoeck et al., 2000; Wang et al., 2000; Lee et al., 2003a; Mebrahtu et al., 2004; Seguin et al., 2004; Primomo et al., 2005; Riedl et al., 2007; Morrison et al., 2008; Tepavčevič et al., 2010; Berman et al., 2009, 2010, 2011; Zhang et al., 2012; Mo et al., 2013).

Kim et al. (2012) compared the isoflavone content of 204 soybean germplasms from America, China, and Korea, classified into three groups based on 100-seed weight: small seeded $(<13 \mathrm{~g})$, medium seeded $(13-24 \mathrm{~g})$, and large seeded $(>24 \mathrm{~g}$ ) varieties. Small soybean seeds are commonly used for soybean sprout production, and medium and large seeds are used for soybean curd, soy milk, cooking with rice or vegetables, and various soybean pastes. The total isoflavone content of the soybeans were from $682.4 \mathrm{mg} / \mathrm{kg} \mathrm{dw}$ to $4,778.1 \mathrm{mg} / \mathrm{kg} \mathrm{dw}$, a sevenfold difference. The small seeded varieties had the highest average total isoflavone concentration $(2,520 \mathrm{mg} / \mathrm{kg}$ $\mathrm{dw}$ ) of the three seed size groups. A decade later, based on studies on 66 soybean varieties, Kim et al. (2003) concluded that large soybeans contain slightly less total isoflavone (daidzein + genistein) than soybeans of medium or small size. The mean level of total isoflavones in the studied bean seeds was $1,209 \mathrm{mg} / \mathrm{kg} \mathrm{dw}$ (range 247 to 2,256 mg/kg dw), a ninefold difference.

Maturity class. Genetic adaptation to a particular photoperiod limits the geographic distribution of a variety to a narrow belt of latitude (about $200 \mathrm{~km}$ ), which have the characteristics to which the variety has been adapted (Scott \& Aldrich, 1970). Hence, for every soybean-growing area there is an optimum maturity class. No such limitations exist with longitude. Whereas an early study by Tsukamoto et al. (1995) concluded that seeds from soybeans of lower maturity classes contain less isoflavones than seeds of soybeans of higher maturity classes (closest to the equator), other investigators have reported the reverse (Mebrahtu et al., 2004) or found no correlation between maturity class and content of total isoflavones (Carrão-Panizzi \& Kitamura, 1995; Wang et al., 2000; Seguin et al., 2004; Tepavčevič et al., 2010). If there were a relationship between the maturity class and isoflavone content, it might not be with total isoflavones but with individual isoflavone conjugates (Wang et al., 2000).

Seed-coat colour. Beans are frequently classified according to the colour of the seed coat, a characteristic that is genetically determined. Malenčić et al. (2012) analysed eight coloured soybean varieties from central Europe for polyphenol and antioxidant content, including the isoflavones. There was no clear correlation 
between seed coat colour and isoflavone content, which is a conclusion shared by Kumar et al. (2010). However, some investigators claim that green-coated (Kim et al., 2003) or yellow-coated soybeans ( $X U \&$ Chang, 2008b) contain more isoflavones than other coloured forms. Apparently, the link between seed-coat colour and total isoflavone content is not very strong.

Site of cultivation. There is extensive data showing that the isoflavone content of soybeans depends on environmental factors, one of which is the location where the crop is grown. Thus, cultivating a particular soybean variety at different sites during the same period may result in significantly different isoflavone levels in the seeds harvested at the various sites (Eldridge \& Kwolek, 1983; Wang \& Murphy, 1994a; Tsukamoto et al., 1995; Hoeck et al., 2000; Lee et al., 2003b; Seguin et al., 2004; Primomo et al., 2005; Riedl et al., 2007; Berman et al., 2009, 2010, 2011).

Many investigators have noted a genotype $\times$ location interaction. For example, Riedl et al. (2007) reported a fivefold range in total isoflavone content $(1,573-7,710$ $\mathrm{nmol} / \mathrm{g}$ ) between seeds from the various location-cultivar combinations in their study. The variation was due both to genotype and to location, as well as the interaction between those factors (Riedl et al., 2007).

Year of cultivation. There is an extensive set of data demonstrating that the isoflavone content of soybeans depends on the season of soybean cultivation (Eldridge \& Kwolek, 1983; Wang \& Murphy, 1994a; Carrao-Panizzi \& Kitamura, 1995; Hoeck et al., 2000; Lee et al., 2003a; Mebrahtu et al., 2004; Seguin et al., 2004; Harrigan et al., 2010b). Season of cultivation is a proxy for environmental conditions, including climatic conditions.

In the study of Seguin et al. (2004), total isoflavone concentration was on average $40 \%$ higher in 2003 than in 2002, with the 2002 season being characterised by above average temperatures and severe drought. It is possible that the relatively low isoflavone content this year was attributable to climatic conditions with lower precipitation than usual under the reproductive phase and a bit warmer than usual.

Temperature and water availability. Temperature and water regimes are two of the most significant abiotic factors influencing isoflavone accumulation in soybeans. While the effect of temperature has been widely studied, much less is known about the effect of water scarcity. To identify factors affecting isoflavone accumulation in soybean seeds, Tsukamoto et al. (1995) used temperature-controlled growth cabinets to study the influence of the temperature during seed development on isoflavone content. They noted that the isoflavone content decreased in seeds after growth at a high temperature for all soybean varieties tested. A general decrease was observed for all isoflavones, rather than being restricted to a single or a few molecular species, an observation confirmed by Caldwell et al. (2005). Support for lower isoflavone concentrations at higher temperatures has been obtained based on controlled environments (Caldwell et al., 2005; Lozovaya et al., 2005; GutierrezGonzalez et al., 2009) and on field trial data (Carrão-Panizzi et al., 1999; Primomo et al., 2005). The influence of temperature can be rather strong. In the experiment of Caldwell et al. (2005), a five degree increase in cultivation temperature for soybeans 
from $18^{\circ} \mathrm{C}$ to $23^{\circ} \mathrm{C}$ during seed development decreased the total isoflavone level by $30-65 \%$, and a ten degree increase with as much at $90 \%$.

A couple of investigators have claimed that early sowing of soybeans results in lower isoflavone content in the harvested crop (Tsukamoto et al., 1995; Aaussenac et al., 1998). However, this finding could not be confirmed by others (Bennett et al., 2004). It was proposed by Tsukamoto et al. (1995) that the early sowing might have led to an earlier seed setting and harvest, resulting in higher temperatures during seed setting and lower isoflavone content in cotyledons. Instead, Aussenac et al. (1998) suggested that the reduced isoflavone content because of early sowing depended on the seed variety (maturity class).

Studies on the relationship between availability of water and isoflavone concentration in the studied plant are less easy to interpret. In carefully controlled greenhouse experiments with soybean, Lozovaya et al. (2005) noted that bean grown under high soil moisture conditions contained higher isoflavone concentrations than soybeans grown under less soil moisture. Gutierrez-Gonzalez et al. (2010) studied the influence of several intensities of water stress at various critical stages for soybean seed development in both field and experimental conditions (controlled environments). Only long-term progressive drought, spanning most of the seed developmental stages, significantly decreased isoflavone content in seeds in a stressintensity dependent manner. The stress-dependent reduction in isoflavone content seemed to be genotype-dependent, possibly correlated to less isoflavone synthase 2 being present.

Stress conditions. Growing plants are exposed to both abiotic and biotic stress. Not all interactions between a plant and other organisms result in stress. For example, for bacteria, there are both beneficial interactions (e.g., with bacteria participating in nitrogen fixation in legumes) and those that are considered neutral or even harmful. The symbiotic bacteria/plant relationship in nitrogen fixation is one of the beneficial interactions and is initiated when the participating microorganism sense complex cocktails of sugars, flavones, or isoflavones, which are perceived as Nod-gene inducers in Rhizobium bacteria. For soybean, the isoflavones genistein and daidzein released by plant roots induce the expression of Nod and host-specific genes in the bacteria (Kosslak et al., 1987). Thus, in studies on soybean roots transformed by Agrobacterium rhizogenes (producing "hairy-roots"), genistein has been demonstrated to significantly increase Nod and nitrogen fixation, as well as to improve growth of roots and shoots (Dolatabadian et al., 2013).

However, a plant perceives stress when attacked by fungi or bacteria by other routes; under such circumstances, isoflavone production might be promoted. For example, Morris et al. (1991) reported that isoflavone and isoflavone glucoside production is stimulated when soybeans are exposed to the potential pest Phytophthora megasperma f.sp. glucinea. Daidzin, malonyl-daidzin, glycitein-7-O- $\beta$ glucoside, genistin, and malonyl-genistin accumulated in the boarder zones surrounding necrotic lesions from the Phytophthora attack, whereas another phytoalexin, glyceollin, was found in the centre of the lesion. However, the role of the isoflavones in defence against Phythophthora megasperma is unclear, as in vitro 
studies demonstrated daidzein, formononetin, and genistein not to inhibit growth of the pest. Induction of isoflavones by biotic stress has subsequently been confirmed by several investigators (Graham \& Graham, 1996; Algar et al., 2013; Lee et al., 2013).

Studies on soybeans have demonstrated that germination of seeds enhance their isoflavone content (Huang et al., 2014). This is a reaction common for phytoalexins. Dutch investigators germinated soybean seeds and concomitantly challenged with Rhizopus microsporus var. oryzae to study the stress reaction in the plant. Exposure to the Rhizopus organism drastically changed the orchestrated chemical defence with the formation of phytoalexins belonging to the subclasses pterocarpans and coumestans and by prenylating various isoflavonoids (Simons et al., 2011). The total isoflavonoid content increased by a factor of 10-12 on dw basis after nine days, which was suggested to be ascribed to de novo synthesis. After the induction, the content of prenylated isoflavones reached $0.30 \mathrm{mg}$ daidzein equivalents $/ \mathrm{g}$. These defencerelated changes could be detected as a gradual increase in agonistic activity of plant extracts toward ERs $\alpha$ and $\beta$, with a more pronounced activity toward ER $\beta$.

Of abiotic stressors, such as wounding, abnormal light, soil, and climatic conditions (the latter includes water stress), several are known to influence the isoflavone content of legumes. Phommalth et al. (2008) observed that light stress under soybean seed germination increased the isoflavone concentrations 1.5 times compared with seeds grown under dark conditions.

Mineral availability in the soil might create a stress condition for cultivated plants. This potential problem is frequently tackled by fertilisation. Vyn et al. (2002) demonstrated that potassium fertilisation increased the daidzein, genistein, and total isoflavone concentration in soybeans cultivated on low potassium soils. Effects on isoflavone contents were marginal on medium- to high-testing potassium soils. Because climatic conditions are not constant but specific year-by-year and locality-bylocality, effects of long-term drought (also called water stress) (Gutierrez-Gonzalez et al., 2010) have already been mentioned in connection with the discussion on the effects of season of cultivation.

Graham and Graham (1996) attempted to separate and characterise the specific contributions of light, wounding, and a Phytophthora cell wall glucan elicitor to the regulation of phenylpropanoid defence responses in soybean cotyledon tissues. Whereas light specifically triggered the accumulation of malonylglucosyl conjugates of genistein, Phytophthora cell wall glucan selectively induced the accumulation of daidzein conjugates, the first committed precursor of the phytoalexin glyceollin.

Breeding of soybeans for other characteristics than those related to the stress response of the plant is not expected to have any major influence on the isoflavone content of the new variety. In agreement with this assumption, Whent et al. (2011) found no alteration in the isoflavone level in new cultivars developed for having low lipoxygenase-1 content. In addition, several research teams reported no alteration in the total daidzein, total genistein. and total glycitein in soybeans being genetically modified for herbicide tolerance, insect resistance, and modified fatty acid profile (Padgette et al., 1996; Taylor et al., 1999; Mc Cann et al., 2005; Lundry et al., 2008; Berman et al., 2009, 2010; Zhou et al., 2011a; Lepping et al., 2013; Fast et al., 2015). 
All the factors mentioned above as having a bearing on the isoflavone concentration in legumes and other plants will not act independently of each other but they interact and influence each other, which has been pointed out by several researchers, including Hoeck et al. (2000), Lee et al. (2003a), Seguin et al. (2004), and Mebrahtu et al. (2004). The issue can be summarised by Wang and Murphy (1994a) who measured the amount of twelve isoflavones in eight American and three Japanese soybean varieties, noting a variation in total isoflavone content from 1,175 to $3,303 \mathrm{mg} / \mathrm{kg}$ (almost $300 \%$ ) in one variety of soybean over a three-year period, and from 1,176 to $1,749 \mathrm{mg} / \mathrm{kg}$ among locations within the same year. In agreement with these observations, Hoeck et al. (2000) reported significant genotype, genotype $x$ year, genotype $x$ location, and genotype $x$ year $x$ location interactions for total isoflavone and pattern.

Mebrahtu et al. (2004) studied vegetable soybean genotypes (in total 31 genotypes, maturity classes III to $\mathrm{VI}$, cultivated in three replications during three years) and determined the largest contribution to the variation in genistein, daidzein, and total isoflavone content. From these findings, it was concluded that genotype and genotype $x$ year interaction gave the most significant contribution. Another finding was that clear differences were observed among the maturity groups for genistein, daidzein, and total isoflavones (but not for glycitein), with the content being lower in the higher maturity classes. Thus, selection for high genistein, daidzein, and total isoflavones should focus on early maturing genotypes. One of the leading experts in isoflavone analysis, Dr Murphy, has stated, "In my own experience with soy isoflavones, we observe that the levels in soybeans, even from the same field grown all in the same year, may vary in concentrations by a factor of five" (Homsey, 1999).

\subsubsection{Chemical analytical methodology}

An important step in the chemical analysis of isoflavones in foodstuffs is their extraction from the food matrix. There is a requirement to have an as complete extraction as possible, but also to have an extraction that does not modify the chemical structures of the native isoflavones. These two requirements are not always easy to fulfil in a single extraction and sample clean-up procedure. Therefore, many investigators have chosen to analyse only for total isoflavones, and thus neglected to identify and quantify the various types of isoflavone in the food. Limiting the analysis to only total isoflavones will make the extraction less critical but not totally unproblematic. It is then possible to combine an efficient extraction (in diluted alcohol or acetonitrile, or at alkaline conditions) of the various isoflavones with a step of hydrolysis (using enzymes or acid conditions) that cleaves isoflavone conjugates and allows the aglycons to be quantified. The official methods for determining isoflavone content in food uses both acetic acid and sodium hydroxide for the extraction of glycosides and aglycones (AOAC, 2005).

It was fairly early recognised that if the extraction of isoflavones from legumes (soybean) take place at too high temperatures, there is a risk that the native plant constituents may be broken down. Thus, Kudou et al. (1991) noted that when 
extraction took place at $80^{\circ} \mathrm{C}$ instead of at room temperature, the malonylated (and to some extent the acetylated) isoflavones were converted to the aglycones/glycoside forms. This observation was confirmed by Barnes et al. (1994), Coward et al. (1998), and Aussenac et al. (1998). Aussenac et al. (1998) reported that malonyl daidzin and malonyl genistin were the major constituents of the extracts at $20^{\circ} \mathrm{C}$, whereas the major components in extracts at $80^{\circ} \mathrm{C}$ were daidzin and genistin. A partial conversion to glycoside forms was already observed at $60^{\circ} \mathrm{C}$. Thus, it seems clear that artefacts may occur at non-appropriate extraction conditions.

Identifying individual native isoflavones is a more demanding challenge. It is not only required to perform the extraction at acceptable temperatures, but it is also necessary to use conditions that do not degrade isoflavone conjugates. There is a risk that the isoflavone content is underestimated if too diluted hydrochloric acids are used in the extraction process during too short a time because of an incomplete cleavage of glycoside (Schwartz et al., 2009). Moreover, there is a risk with using too strong acids or very long incubation times to enhance the yield of isoflavones, as glucoside isoflavones may be hydrolysed to aglycones and genistein may partially be degraded (Müllner \& Sontag, 1999, Schwartz et al., 2009).

Lin and Giusti (2005) pointed out that choosing a solvent system that can accurately quantify the amounts of individual isoflavones present in legumes is paramount. The investigators compared frequently used solvent systems (83\% acetonitrile, $80 \%$ methanol, and $58 \%$ acetonitrile) with and without acids. The authors then evaluated the effects of polarity and acidity on the recovery of isoflavones from soybeans. Solvents with a higher polarity ( $58 \%$ acetonitrile) extracted a significantly higher amount of total isoflavones. Acidification risked significantly reduced recovery of malonylglucosides and isoflavone transformation. Among the six solvent systems examined, a $58 \%$ acetonitrile aqueous solution without acid was the best for extraction of isoflavones from soybeans.

Quantification of isoflavones in some foods might be improved if the fat content are reduced. In such cases, it should be recognised that the method of removing lipids might be critical (Klump et al., 2001; Kao \& Chen, 2002; Oomah, 2002).

Chukwumah et al. (2007a) compared four extraction methods applied in the chemical analysis for isoflavones and trans-resveratrol. Microwave-assisted sonication and Soxtec methods extracted significantly higher amounts of the phytochemicals compared with stirring and sonication. At the same time, it was noted in studies on peanuts that the extraction was much more efficient on defatted peanuts than on non-defatted peanuts. It was speculated that the high levels of the isoflavones obtained with the first two of these methods might be attributed to heat-induced conversion of conjugated glycosides to aglygones. Thus, the microwave-assisted sonication and Soxtec methods may be used for quantification of total isoflavones, whereas sonication or stirring may be methods of choice for quantification of isoflavone composition (aglycones and glycoside conjugates) in peanuts.

Because there will be certain losses during a multistep extraction of isoflavones, internal standards should be used for precise isoflavone quantification. Song et al. (1998) suggested 2,4,4' -trihydroxy deoxybenzoin as standard. 
The total isoflavone content of a sample is not the simple sum of the masses of the twelve isomers (malonyldaidzin, malonylgenistin, malonylglycitin, acetyldaidzin, acetylgenistin, acetylglycitin, daidzin, genistin, glycitin, daidzein, genistein, and glycitein). The normalised concentration of each isoflavone should be summed up, i.e. the mass of each isoflavone form should be multiplied with the ratio of its aglycone molecular weight to the molecular weight of the individual form (which is $270 / 432$ to give free genistein from genistin) and then summed up.

\subsubsection{Studies on isoflavone contents in foodstuffs}

In earlier work, Mazur, Adlercreutz, and co-workers analysed an extensive set of foods for their content of phytoestrogens in order to use the data in epidemiological studies looking at the connection between the dietary pattern and diseases (e.g., cancer and coronary heart disease) (Mazur \& Adlercreutz, 1998; Mazur et al., 1998a, 1998b). Phytoestrogens looked for in these studies included isoflavonoids, coumestans, and lignans. Data collections on the content of phytoestrogens in foods have subsequently been established by others (Reinli \& Block, 1996; Horn-Ross et al., 2000; Thompson et al., 2006; Kuhnle et al., 2008a, 2008b, 2009a, 2009b, Clarke et al., 2013a), whereas in a review Schwartz et al. (2009) made an inventory of available phytoestrogen databases.

\section{Effects of phytoestrogens}

This report does not consider biological effects resulting from the exposure to isoflavones. However, it should be recognised that a general problem in studying the influence of hormonally active compounds on humans and experimental animals is that the diet needed to support health and well-being in the studied subjects may contain natural compounds or xenobiotics having activity mimicking/interfering with that of the compound studied (such as $17-\beta$-estradiol).

Epidemiological studies indicate that Japanese women consuming a traditional diet, thereby having a high dietary intake of isoflavones or flavonols, or both, have a low incidence of breast cancer, cardiovascular disease, osteoporosis, and climacteric symptoms. The mechanism for this reduced risk is not clear but it has been proposed that it could be the result of inhibition of low density lipoprotein oxidation by isoflavones. Genistein also appears to improve plasma lipids, resulting in lowered LDL cholesterol, the ratio of total cholesterol to HDL cholesterol, and the ratio of LDL to HDL cholesterol in pre-menopausal women.

Oestrogen deficiency in post-menopausal women can lead to unpleasant symptoms, such as hot flushes and vaginal dryness, with a long-term increased risk of bone loss in addition to cardiovascular disease. Soybean isoflavones have been shown to positively help maintenance of bone mass in rodent, daidzein being more efficient than genistein. Against this background, it has been claimed that an isoflavone-rich diet may help approximately two thirds of post-menopausal women to better cope with oestrogen-related effects such as hot flushes, in addition to potentially reducing the risk of cardiovascular disease, which is elevated post-menopause. Another 
ongoing discussion is whether a high soy diet can improve episodic and semantic memory, which was indicated in a study on young male and female volunteers (File et al., 2001).

However, a diet rich in phytoestrogens might not only have positive effects on human health. During critical stages of infant development, exposure to high concentrations of phytoestrogens may possibly result in adverse health effects. In this context, it should be reminded that in addition to having estrogenic activity, genistein, at even higher concentrations, inhibits DNA topoisomerase and tyrosine protein kinase (Akiyama et al., 1987). Moreover, it possesses antioxidant and cell cycle inhibiting activity. Kinase inhibition is generally regarded as being specific for tyrosine kinases (e.g., epidermal growth factor receptor), although at higher concentrations genistein also inhibits protein histidine kinase. Other isoflavones, such as daidzein, do not inhibit tyrosine kinase activity. In several cell systems in which genistein inhibits growth, it does not appear to induce phosphorylation of EGF receptors or other tyrosine kinase substrates. In such cases, it has been suggested that the isoflavone might inhibit cell growth by modulating transforming growth factor (TGF) $\beta_{1}$ signalling pathways. It seems likely that the timing of high exposures to phytoestrogens may be important.

Estimating the phytoestrogen exposure from information on the concentration of various phytoestrogens in different foodstuffs is not straightforward. The majority of phytoestrogens occur in foodstuffs as inactive compounds. Regarding isoflavones, the glycosylated forms in the legume products need to be hydrolysed by $\beta$-glucosidases to be converted to active aglyconic forms. This step is usually not rate-limiting. Therefore, the bioavailability of isoflavones in soy is not significantly different when the isoflavones are consumed as aglycones or glucosides. Following the ingestion of a controlled quantity of soy, there is a variable individual metabolic response, with up to a 1000-fold variation in subsequent isoflavone excretion (Murkies et al., 1998). It has been hypothesised that the composition of the intestinal flora, intestinal transit time, and variability in redox potential of the colon might all contribute to this variability in humans (Setchel et al., 1984). Because the estrogenic activity of the isoflavones depends on the type of aglycone, the isoflavone profile in the diet is important. Genistein is found to have higher affinity for ERs in comparison with daidzein and glycitein (Potter et al., 1998).

Although phytoestrogens compete effectively with estradiol for receptor binding at nanomolar (10-9M) concentrations, in higher concentrations (that is $10-{ }^{6} \mathrm{M}$ ) they inhibit several enzymes, including protein kinase and tyrosine kinase. This may contribute substantially to certain clinical effects, particularly their anti-proliferative actions (Whitten, 1998).

A study dating back forty years ago concluded that phytoestrogens have some 1000-fold weaker effects compared with those of $17 \beta$-estradiol (Lindner, 1976).

Isoflavones in food

The major dietary sources of isoflavonoids for humans are soy products. One gram of powdered soybean chips contains nearly $800 \mu \mathrm{g}$ of daidzein and over $500 \mu \mathrm{g}$ of 
genistein (primarily as glycosides). In contrast, one gram of soy protein has approximately $150 \mu \mathrm{g}$ of daidzein and $250 \mu \mathrm{g}$ of genistein. Highly processed soy products (such as miso and soy sauce) contain lower levels of genistein than does tofu, the major source of isoflavones in the Asian diet.

Eating a soy-rich diet, ingested isoflavone levels can be very high in humans, as determined by urinary excretion. The level of equol in urine can be approximately 100-fold higher in adults having a soybean-rich diet than in adults who consume little soy products in their diet. A high dietary consumption of genistein has been linked to several potential health benefits, including reduced incidence of breast cancer (Lee et al., 1991) and reduced mortality from prostate cancer. Similar observations have been reproduced in many, but not all, subsequent studies undertaken up to the present day. There are also animal studies pointing in the same direction. Despite the large number of studies supporting cancer chemoprevention by genistein, some studies have suggested a potential for opposite effects (e.g., increased number of carcinogeninduced aberrant crypt foci in the colon of rats fed genistein and induced chromosomal aberrations in human peripheral lymphocytes).

In the following sections, the available information on isoflavone contents in foodstuffs of various types is reported. The individual sections (3.1.4-3.1.13) present the isoflavone content of the various types of foodstuff. For many food groups, data are presented as published, i.e. some investigators have given the content as $\mathrm{mg} / \mathrm{kg}$

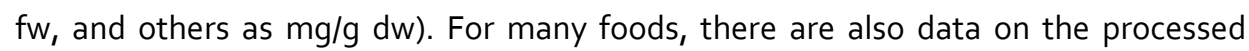
food. The influence of food processing on the isoflavone content in the various foodstuffs is discussed in section 3.1.14.

From what has been stated above about the role of many phytoestrogens in plants as having a role in the orchestrated defence of the plant, it is understandable that a considerable variation in the concentration of isoflavones have been reported between samples. This variation is illustrated by the studies of Kuhnle et al. (2009b) that analysed nine types of food from different sources and countries of origin for their isoflavone and lignan levels. The average detected range of phytoestrogen content between samples was threefold with an average coefficient of variation for isoflavones of $37 \%$ and lignans of $33 \%$.

\subsubsection{Isoflavones in products of animal origin}

Although products of animal origin are not expected to contain endogenously produced phytoestrogens, they may still contain such compounds because of carryover from the feed. The phytoestrogens in ruminant feedstuffs are mainly isoflavones, coumestans, and lignans. The isoflavones are predominantly found in legumes used as animal feedstuffs. The content varies between plant species, with highest levels occurring in red clover. Coumestans are mainly found in the feed Lucerne and in small amounts in white clover (Saloniemi et al., 1995). The primary plant lignans are found in feeds coming from cereals, legumes, and oilseed crops used in concentrates (Thompson et al., 1991). However, it is not only the plant species that decides on the phytoestrogen profile and content but also the plant tissue used and 
maturity of the plant might be crucial in this respect. In a study performed in Illinois, USA, above-ground parts and flower heads of cultivated red clover were collected over one growing season and analysed for isoflavones (Booth et al., 2006). Daidzein and genistein contents peaked around June to July, whereas formononetin and biochanin A contents peaked in early September. Apparently, time of harvest will influence the content of these phytoestrogens in the feed.

A couple of investigators have studied the carryover of phytoestrogens from the feed supplied to diary cows to the milk collected from the animals. Steinshamn et al. (2008) compared the level of phytoestrogens in milk from dairy cows fed silage of white and red clover. The intake of isoflavones (which in grassland legumes such as clover are mainly formononetin and biochanin A) was several times greater in red clover silage than in white clover silage, whereas intake of lignans was greater from white clover silage. The red clover diet yielded milk with a higher content of isoflavones, whereas milk from white clover silage diets had a higher content of the mammalian lignans enterodiol and enterolactone.

Andersen et al. (2008) studied the influence of feeding Lucerne and grass/clover silage to dairy cows on the concentration of phytoestrogens in the bovine milk. Milk and pooled feed samples were analysed for the concentration of isoflavones, coumestans, and lignans. The content of isoflavones was higher in the grass/clover silage than in the Lucerne silage. The situation was the reverse for coumestrol. The concentration of coumestrol and secoisolariciresinol increased with the proportion of the fed being Lucerne, whereas the concentration of isoflavones was similar across the diet. The concentration of formononetin, daidzein, and equol in the milk ${ }^{5}$ was significantly higher for cows fed the grass/clover diet than the Lucerne diet. In particular, the concentration of equol was 62-291 times higher for grass/clover silage. Thus, a high concentration of isoflavones, particularly equol, and a low concentration of coumestrol were measured in the milk from the grass/clover silage diet. In contrast, a low concentration of isoflavones and a high concentration of coumestrol were measured in the milk form the Lucerne silage diets. However, it should be stressed that the concentration of phytoestrogens in bovine milk is low compared with food sources rich in those phytoestrogens. The high concentration of equol could in some foods of animal origin possibly be of interest.

The isoflavonoids equol, formononetin, daidzein, genistein, biochanin $\mathrm{A}$, and $\mathrm{O}$ demethylangolensin were analysed in skimmed milk from commercial Finnish milk cartons (Hoikkala et al., 2007). In organic skimmed milk, the average amount of equol was $411 \mathrm{ng} / \mathrm{mL}$, whereas only traces of formononetin and daidzein were detected. In sharp contrast, conventionally produced skimmed milk contained on average $62 \mathrm{ng}$ equol/mL, and no formononetin or daidzein. Because the difference might have been entirely due to different feeds having been given to the cows, these data distinguish nothing about the potential influence of the different agricultural systems.

5 When consumed by livestock or humans, isoflavonoids are metabolised by the rumen and gut flora (formononetin to daidzein and further to equol), whereas biochanin $A$ is metabolised to genistein and further to p-ethyl phenol. 


\section{Seafood}

Only a handful of publications have reported on the level of the isoflavone aglycones daidzein, genistein, glycitein, biochanin A, and formononetin in seafood. No data were found on shellfish. The fishes analysed were cod (microwaved), salmon, and tuna. Whereas cod and salmon only contained traces of the studied isoflavone aglycones, if any at all (Kuhnle et al. 2008a), tuna contained $4.13 \mathrm{mg}$ daidzein and 7.3 mg genistein per $\mathrm{kg}$ fw. No biochanin A and formononetin were found (Horn-Ross et al. 2000). Canned tuna contained low levels of most of these isoflavone aglycones on a $\mathrm{fw}$ basis: $0.007 \mathrm{mg} / \mathrm{kg}$ daidzein, $0.016 \mathrm{mg} / \mathrm{kg}$ genistein, $0.001 \mathrm{mg} / \mathrm{kg}$ glycitein, and $0.022 \mathrm{mg} / \mathrm{kg}$ formononetin (Thompson et al. 2006), as did the brine from those cans (Kuhnle et al. 2008a). Clarke et al. (2013a) analysed an unspecified sushi dish for isoflavones but could only identify very low levels of genistein and formononetin.

\section{Dairy products}

With few exceptions, isoflavone levels are very low in dairy products and rarely reached $0.1 \mathrm{mg} / \mathrm{kg} \mathrm{fw}$ for any of the isoflavones analysed (Table 2). The exception was a poorly defined sample of ice cream that contained close to $0.5 \mathrm{mg} / \mathrm{kg}$ of total genistein and total daidzein. It is suggested that this sample possibly could have contained some soybean material.

Poultry and egg

Only limited information is available on the isoflavone content of poultry products (Table 2). Roasted chicken contained low levels of total genistein (0.01-0.02 mg/kg $\mathrm{fw}$ ) (Kuhnle et al., 2008a). Comparable levels have been found in chicken skin that also contains equally low levels of total daidzein, glycitein, and biochanin A. No data on the isoflavone content in fresh chicken meat were found. Eggs contain nondetectable to low levels ( $0.07 \mathrm{mg} / \mathrm{kg} \mathrm{fw}$ ) of all isoflavones analysed. However, individual samples have shown somewhat higher concentrations of total biochanin A (n.d.-0.21 mg/kg fw) and total daidzein (n.d.-0.28 mg/kg fw) (Horn-Ross et al., 2000; Kuhnle et al., 2008a).

\section{Meat products}

Isoflavone levels in meat products are low (Table 2). Kuhnle and co-workers (2008a) reported on the level of isoflavones in roasted and grilled meat from cow, pig, and lamb. Roasted beef contains traces of biochanin $A$, roasted lamb nondetectable to $0.03 \mathrm{mg}$ total glycitein and traces of biochanin $\mathrm{A}$, and grilled pork $0.03 \mathrm{mg}$ total daidzein $/ \mathrm{kg} \mathrm{fw}$. Pork crackling contained $0.02 \mathrm{mg}$ total genistein and biochanin A, and 0.04 total glycitein per $\mathrm{kg} \mathrm{fw}$. The meat product containing most isoflavones was corned beef but levels were still rather low: $0.11 \mathrm{mg}$ total genistein, $0.04 \mathrm{mg}$ total glycitein, and $0.01 \mathrm{mg}$ biochanin A per $\mathrm{kg}$ fw (Kuhnle et al., 2008a). Also, ham contained some of these isoflavones: $0.006 \mathrm{mg}$ total genistein, 0.008 total daidzein, $0.001 \mathrm{mg}$ total glycitein, and $0.003 \mathrm{mg}$ formononetin per $\mathrm{kg}$ fw (Thompson et al., 2006). 
Table 2: Isoflavone content $(\mathrm{mg} / \mathrm{kg}$ or $\mathrm{mg} / \mathrm{L} \mathrm{fw}$ ) in products of animal origin

\begin{tabular}{|c|c|c|c|c|c|}
\hline $\begin{array}{l}\text { Type of food and } \\
\text { processing }\end{array}$ & Genistein & Daidzein & Glycitein & Biochanin A & Formononetin \\
\hline \multicolumn{6}{|l|}{ Dairy products } \\
\hline $\operatorname{Milk}(2,6)$ & n.d.-0.04 & n.d.-0.02 & n.q. -0.03 & n.d.-0.01 & n.q. -0.005 \\
\hline Milk, skimmed $(2,6,7)$ & $0.01-0.06$ & n.q. -0.08 & n.q. & n.q. -0.02 & n.d. -0.006 \\
\hline Milk powder (2) & $0.02-0.05$ & n.q. -0.01 & n.q. -0.02 & n.d.-n.q. & n.q. \\
\hline Milkshake (1) & 0.006 & 0.002 & 0.001 & & 0.005 \\
\hline Butter (2) & $0.04-0.06$ & traces & traces & $0.02-0.06$ & traces \\
\hline Cream (2) & traces-0.02 & traces-0.01 & traces & traces-0.05 & traces \\
\hline Crème fraiche (2) & n.q. & n.q. & n.q. & n.q. & n.q. \\
\hline Cheese (2) & n.d.-o.o9 & traces & n.d. -0.03 & traces-0.06 & traces-0.03 \\
\hline Cheese, cottage type (2) & traces-0.02 & traces & n.d.-traces & n.d. -0.07 & traces \\
\hline Quark (2) & n.q. & n.q. & n.q. & n.d. & n.q. \\
\hline Ice cream, various types (2) & $0.01-0.13$ & n.q. -0.06 & n.q. -0.02 & n.d.-n.q. & n.d.-n.q. \\
\hline Ice cream (3) & 0.435 & 0.478 & & n.d. & n.d. \\
\hline Ice cream dessert (2) & n.q.-o.01 & n.q. & n.q. & n.d. & n.q. \\
\hline Goats milk (2) & n.d. & n.q. & n.q. & n.d. & n.q. \\
\hline \multicolumn{6}{|l|}{ Poultry and egg } \\
\hline $\operatorname{Egg}(2,3)$ & traces-0.07 & traces-0.28 & n.d.-0.02 & n.d. -0.21 & traces-0.02 \\
\hline Chicken, breast, roast (2) & $0.01-0.02$ & n.q. & n.q. & n.q. & n.q. \\
\hline Chicken, skin (2) & $0.01-0.02$ & n.q. -0.02 & n.q. -0.02 & $0.02-0.03$ & n.d.-n.q. \\
\hline \multicolumn{6}{|l|}{ Meat products } \\
\hline Beef, corned type (2) & 0.11 & n.q. & 0.04 & 0.1 & n.d. \\
\hline Roast beef ( 2 ) & n.d.-traces & traces & traces & traces-0.02 & n.d. -traces \\
\hline Marrow (4) & n.d. & n.d. & & & \\
\hline Marrow. cooked (4) & n.d. & n.d. & & & \\
\hline Lamb, fat and lean, roast ( 2 ) & n.d. & n.q. & n.q. -0.03 & n.q. -0.01 & n.d.-n.q. \\
\hline Lamb, liver, grilled (2) & n.q. & n.q. & n.q. & 0.1 & n.q. \\
\hline Pig liver, grilled (2) & n.q. & 0.03 & n.q. & n.d. & n.q. \\
\hline Pork, lean or fat (2) & n.d. & n.q. & n.q. & n.d.-0.02 & n.d. \\
\hline Pork crackling (2) & 0.02 & n.d. & 0.04 & 0.02 & n.d. \\
\hline $\operatorname{Ham}(1)$ & 0.006 & 0.008 & 0.001 & & 0.003 \\
\hline Sausage, hot dog (1) & 0.005 & 0.001 & 0.001 & & 0.006 \\
\hline Sausage, breakfast type (1) & 0.002 & n.q. & n.q. & & 0.002 \\
\hline
\end{tabular}

Note: $\quad$ n.d. = non-detectable; n.q. = non-quantifiable.

Source: (1) Thompson et al., 2006; (2) Kuhnle et al., 2008a; (3) Horn-Ross et al., 2000; (4) Liggins et al., 200ob; (5) Kuhnle et al., 2007; (6) Antignac et al., 2004; (7) Hoikkala et al., 2007.

\subsubsection{Isoflavones in beverages}

The isoflavone content in beverages is summarised in Table 3. The Table gives information on the total content of the five isoflavone aglycones genistein, daidzein, glycitein, biochanin A, and formononetin. Because no isoflavones were found in strong alcoholic beverages (e.g., gin and whiskey) and intermediate alcohol content drinks (sherry, red and white wine) and low alcohol content beverages (e.g., Horlicks and cider, which only contained $0.01 \mathrm{mg} / \mathrm{kg}$ few biochanin A) all contained low levels of isoflavones, it seems unlikely that the alcohol level influences the content of these compounds in the beverage. Red and white wine contained small amounts of total daidzein, genistein, glycitein, and formononetin, the latter compound being most common. Sherry contained only low levels of biochanin A and formononetin. 
Concerning coffee and tea, there are data both on dried material used to prepare the drinks and on the ready-to-drink beverage. Only the data on the prepared drinks are shown in Table 3. Ground coffee beans of various brands and of various intensities of roasting contained quite different amounts of total genistein $(0.15-5.12 \mathrm{mg} / \mathrm{kg} \mathrm{dw})$, total daidzein (7.48-31.2 mg/kg dw), and formononetin (0.72-89.1 mg/kg) (Mazur et al., 1998a; Alves et al., 2010). Instant coffee powder contained $5.94 \mathrm{mg}$ total genistein, $1.53 \mathrm{mg}$ total daidzein, $1.62 \mathrm{mg}$ total glycitein, and $0.04 \mathrm{mg}$ formononetin per $\mathrm{kg} \mathrm{dw}$ (Kuhnle et al., 2008b). Most high-quality coffee is produced from Coffea arabica beans, whereas quite a proportion of the coffee beans in instant coffee frequently are from Coffea robusta, and there are all qualities in between. Total isoflavone level is sixfold higher in robusta than in arabica coffees, mainly because of formononetin (Alves et al., 2010). The range in total isoflavones in espresso coffee $(30 \mathrm{~mL})$ was between about $40 \mu \mathrm{g}$ based on arabica beans coffee and about $285 \mu \mathrm{g}$ based on robusta beans. Expressos prepared from commercial blends on average contained 6,17 , and $78 \mu \mathrm{g}$ of genistein, daidzein, and formononetin, respectively. During roasting, the content of isoflavones decreases, whereas their extractability increases (especially that for formononetin). The data of Alves et al. (2010) partly disagree with the reports of other investigators on very low isoflavone content in brewed coffee. Perhaps the analytical methodology has not been optimal for formononetin?

Tea leaves contained less isoflavones, $0.15-0.29 \mathrm{mg}$ total genistein, $0.1-0.66 \mathrm{mg}$ total daidzein, and 0.05-0.19 mg biochanin A per kg dw (Mazur et al., 1998a; Clarke et al., 2013a) than ground coffee. However, the brewed beverages contain negligible amounts of isoflavones. The only "tea" reported to contain isoflavone levels worth mentioning is Lapacho tea. However, this herbal tea is not prepared from the leaves of Camellia sinensis, but from the inner bark of the Pau d'arco tree Handroanthus impetiginosus. Lapacho tea contains $0.29 \mathrm{mg}$ total genistein, $0.18 \mathrm{mg}$ total daidzein, $0.36 \mathrm{mg}$ biochanin $\mathrm{A}$, and $0.075 \mathrm{mg}$ formononetin per $\mathrm{kg} \mathrm{dw}$. It is possible that other brewed herbal teas contain comparable low amounts of isoflavones.

The only fruit juices that have been studied for their isoflavone content are orange juice and lemon juice. Both juices are low in isoflavones, lemon juice containing a little more than orange juice. A cocktail having cranberry as an ingredient was reported to contain total genistein $(0.003 \mathrm{mg} / \mathrm{kg} \mathrm{fw})$, daidzein $(0.002 \mathrm{mg} / \mathrm{kg} \mathrm{fw})$, and formononetin $(0.01 \mathrm{mg} / \mathrm{kg} \mathrm{fw})$, whereas no total glycitein was detected (Thompson et al., 2006). 
Table 3: Isoflavone con

$\begin{array}{llllll}\text { Type of food and processing } & \text { Genistein } & \text { Daidzein } & \text { Glycitein } & \text { Biochanin A } & \text { Formononetin } \\ \text { Beer, various types }(1,2) & \text { n.d.-0.02 } & \text { n.d.-0.013 } & \text { n.d.-0.04 } & \text { n.d.-0.06 } & \text { n.d.-0.02 } \\ \text { Cider }(2) & \text { n.d. } & \text { n.d. } & 0.04 & 0.01 & \text { n.d. } \\ \text { Coffee }(2,3,4) & \text { n.d.-0.001 } & \text { n.d.-0.503 } & \text { n.d.-0.002 } & \text { n.d. } & \text { n.d.-0.002 } \\ \text { Coffee, decaffeinated (1) } & \text { n.d. } & \text { n.d. } & \text { n.d. } & \text { n.d. } & \text { n.d. } \\ \text { Gin (2) } & \text { n.d. } & \text { n.d. } & \text { n.d. } & \text { n.d. } & \text { n.d. } \\ \text { Horlicks (3) } & \text { n.d. } & \text { n.d. } & \text { n.d. } & \text { n.d. } & \text { n.d. } \\ \text { Lemon juice (8) } & 0.04 & 0.01 & 0.02 & 0.04 & \text { n.d. } \\ \text { Orange juice }(2,4) & \text { n.d.-0.001 } & \text { n.d.-0.001 } & \text { n.d. } & \text { n.d. } & \text { n.d.-0.001 } \\ \text { Sherry, various types (1) } & \text { n.d. } & \text { n.d. } & \text { n.d. } & \text { n.d.-0.01 } & \text { n.d.-0.02 } \\ \text { Tea (1, 2, 3, 5) } & \text { n.d.-0.03 } & \text { n.d.-0.01 } & \text { n.d. } & \text { o.03 } & 0.001 \\ \text { Tea, licorice }(4) & 0.26 & \text { n.d. } & & \text { n.d. } & 0.999 \\ \text { Tea, green }(1,2,3) & \text { n.d.-0.013 } & \text { n.d.-0.004 } & \text { n.d.-0.04 } & \text { n.d. } & \text { n.d.-0.001 } \\ \text { Whiskey }(2) & \text { n.d. } & \text { n.d. } & & & \\ \text { Wine, red }(1,2) & \text { n.d.-0.025 } & \text { n.d.-0.015 } & 0.003-0.04 & \text { n.d. } & \text { n.d.-0.121 } \\ \text { Wine, white (1) } & 0.016 & 0.007 & 0.001 & & 0.22\end{array}$

tent $(\mathrm{mg} / \mathrm{kg} \mathrm{fw})$ in beverages

Note: $\quad$ n.d. $=$ non-detectable or non-quantifiable.

Source: (1) Thompson et al., 2006; (2) Kuhnle et al., 2008b; (3) Clarke et al., 2013a; (4) Horn-Ross et al., 2000; (5) Mazur et al., 1998a; (6) Kuhnle et al., 2007.

\subsubsection{Isoflavones in fruits and berries}

The content of isoflavones in fruit and berries is reported in Tables 4, 5, 6, and 7. Because published data have been reported both on a fresh weight ( $\mathrm{fw}$ ) and a dry weight ( $\mathrm{dw}$ ) basis and because enough information is not always available to recalculate the data into $d w$, data on the content of isoflavones in fruit given on a dw basis are reported in Table 4 and data given on a fw basis in Table 5 . The data on isoflavone content in berries are given on a dw basis in Table 6 and on a fw basis in Table 7 .

Fruits and berries are well known to contain high amounts of other types of flavonoid than isoflavones, but their contents of isoflavones are low. When measured on a fw basis (Table 4 and 6 ), about $40 \%$ of fruits and $17 \%$ of berries contained no isoflavones. The highest isoflavone content in fresh fruits was found in passion fruit, which contained $0.11 \mathrm{mg}$ total genistein and $0.07 \mathrm{mg}$ total daidzein per $\mathrm{kg}$ fw. Dried fruits such as dates and figs and in particular prunes and raisins contained higher amounts of isoflavones. Thus, raisins were reported to contain up to $1.25 \mathrm{mg}$ total genistein and $0.59 \mathrm{mg}$ total daidzein per $\mathrm{kg}$ fresh fruit, and cooked dried prunes 1.33 $\mathrm{mg}$ total genistein and $0.03 \mathrm{mg}$ total daidzein per $\mathrm{kg}$ fresh fruit.

Screening various other foods than those presented in the Tables above for the presence of daidzein and genistein after an initial step converting the diphenolic glycosides into their respective aglycones, Mazur (1998) reported traces of these isoflavones in bramble (Rubus fructicosus), strawberry (Fragaria $x$ ananassa), and red raspberry (Rubus ideaus) but found nothing in lingonberry (Vaccinium vitis-ideae), 
cranberry (Vaccinium macrocarpum), blackcurrant (Ribes nigrum), redcurrant (Ribes rubrum), otaheite gooseberry (Phyllanthus acidus), avocado (Persea americana), tomato (Lycopersicum esculentum), lychee (Litchi chinensis), papaya (Carica papaya), guava (Psidium guajava), cantaloupe (Cucumis melo cantalupensis), lemon (Citrus limon), orange (Citrus sinensis), and several types of wine.

Table 4: Isoflavone content in fruits, measured as $\mathrm{mg} / \mathrm{kg}$ fresh weight

\begin{tabular}{|c|c|c|c|c|c|}
\hline $\begin{array}{l}\text { Type of food and } \\
\text { processing }\end{array}$ & Genistein & Daidzein & Glycitein & Biochanin A & $\begin{array}{l}\text { Formono- } \\
\text { netin }\end{array}$ \\
\hline Apple (3) & n.d. & n.d. & & & \\
\hline Apple, cooked (3) & n.d. & n.d. & & & \\
\hline Apricot (3) & n.d. & n.d. & & & \\
\hline Cherry (3) & n.d. & n.d. & & & \\
\hline Clementine (3) & 0.029 & 0.0029 & & & \\
\hline Dates, dried $(3,4)$ & $0.034-0.052$ & $0.012-0.018$ & 0.002 & & 0.004 \\
\hline Figs (3) & 0.014 & 0.005 & & & \\
\hline Figs, dried (3) & 0.042 & 0.018 & & & \\
\hline Grapefruit $(4,5)$ & $0.001-0.271$ & $0.001-0.356$ & n.d. & n.d. & n.d.-0.002 \\
\hline Grapefruit, canned (3) & n.d. & n.d. & & & \\
\hline Grapes (3) & n.d. & n.d. & & & \\
\hline Kiwi (3) & n.d. & n.d. & & & \\
\hline Lychees (3) & n.d. & n.d. & & & \\
\hline Mango (3) & 0.032 & 0.038 & & & \\
\hline Mango, canned (3) & 0.013 & 0.009 & & & \\
\hline Melon, cantaloupe $(3,4)$ & n.d. -0.004 & n.d. & n.d.-0.001 & 0.03 & n.d. -0.002 \\
\hline Melon, Galia (3) & 0.008 & 0.002 & & & \\
\hline Melon yellow honeydew (3) & 0.011 & 0.015 & & & \\
\hline Nectarine (3) & n.d. & n.d. & & & \\
\hline Orange $(3,4)$ & n.d.-0.005 & n.d.--0.008 & n.d. & & 0.011 \\
\hline Passion fruit (3) & 0.108 & 0.066 & & & \\
\hline Peach $(3,4,5)$ & n.d.-0.01 & n.d.-0.011 & n.d. & n.d. & n.d. -0.005 \\
\hline Pear (3) & n.d.-0.007 & n.d.- -0.0004 & & & \\
\hline Pears, canned (3) & 0.0104 & 0.002 & & & \\
\hline Pineapple (3) & n.d. & n.d. & & & \\
\hline Plantain (3) & n.d. & n.d. & & & \\
\hline Plantain, cooked (3) & n.d. & n.d. & & & \\
\hline Plum (3) & n.d. -0.074 & n.d.-0.001 & & & \\
\hline Plum, greengage (3) & n.d. & n.d. & & & \\
\hline Pomegranate (3) & n.d. & n.d. & & & \\
\hline Prune (5) & n.d. & n.d. & & n.d. & n.d. \\
\hline Prune, dried (3) & 0.085 & 0.043 & & & \\
\hline Prune, dried (4) & 0.002 & 0.026 & 0.009 & & 0.005 \\
\hline Prune, dried, cooked (3) & 1.333 & 0.031 & & & \\
\hline Pumpkin $(3,4)$ & n.d.-0.002 & $0.001-0.012$ & n.d. & & n.d. \\
\hline Pumpkin pie (4) & 0.003 & 0.002 & 0.002 & & 0.003 \\
\hline Pumpkin seed $(1,2)$ & n.d.-0.05 & n.d. & n.d.-0.02 & n.d.-0.07 & n.d. -0.03 \\
\hline Pumpkin seed, roasted (2) & n.q & n.d. & 0.03 & n.d. & 0.01 \\
\hline Raisins $(4,5)$ & n.d. -0.052 & n.d.-0.015 & 0.01 & n.d. & n.d. -0.004 \\
\hline Raisins (3) & 1.2465 & 0.590 & & & \\
\hline Satsumas (3) & n.d. & 0.008 & & & \\
\hline Strawberry (3) & 0.01 & n.d. & n.d. & 0.05 & n.d. \\
\hline
\end{tabular}

Note: $\quad$ n.d. $=$ non-detectable or non-quantifiable.

Source: $\quad$ (1) Clarke et al., 2013a; (2) Kuhnle et al. 2008b; (3) Liggins et al., 2000a; (4) Thompson et al. 2006; (5) Horn-Ross et al. 2000. 
Table 5: Isoflavone content in fruits, measured as $\mathrm{mg} / \mathrm{kg}$ dry weight

\begin{tabular}{|c|c|c|c|c|c|}
\hline Type of food and processing & Genistein & Daidzein & Glycitein & Biochanin A & Formononetin \\
\hline Apple $(1,2,4)$ & n.d. -0.04 & $0.002-0.11$ & n.d. -0.03 & n.d. -0.32 & n.d.-0.07 \\
\hline Apricot $(1,3)$ & n.d. -0.04 & n.d. -0.03 & n.d. & n.d. & n.d. \\
\hline Apricot, dried $(3,4,5)$ & n.d. -0.198 & n.d.-0.064 & 0.011 & n.d. & n.d. -0.125 \\
\hline Apricot, canned (3) & n.d. & n.d. & & & \\
\hline Banana $(1,3,4,6)$ & n.d. -0.03 & n.d.-0.001 & $0.002-0.03$ & 0.05 & n.d. -0.003 \\
\hline Cherry (1) & 0.03 & 0.06 & 0.17 & 0.05 & n.d. \\
\hline Grapefruit (1) & 0.2 & 0.29 & n.d. & 0.99 & 0.06 \\
\hline Grapes $(1,3)$ & n.d. -0.21 & n.d. -0.06 & n.d. & n.d. -0.08 & n.d. \\
\hline Mango (1) & 0.01 & n.d. & n.d. & 0.05 & n.d. \\
\hline Nectarine (1) & 0.01 & 0.02 & 0.01 & 0.05 & n.d. \\
\hline Orange (1) & 0.09 & 0.25 & n.d. & 0.37 & 0.02 \\
\hline Peach (1) & n.d. & n.d. & n.d. & 0.01 & n.d. \\
\hline Peach, canned (3) & 0.01 & 0.005 & & & \\
\hline Pear $(2,4)$ & n.d.-o.o69 & n.d. -0005 & $0.02-0.201$ & n.d.-0.008 & $0.001-0.002$ \\
\hline Plum $(1,6)$ & n.d.-0.01 & n.d. -0.02 & 0.09 & 0.03 & n.d. \\
\hline Plum, greengage (1) & 0.03 & 0.02 & 0.05 & n.d. & n.d. \\
\hline Pumpkin (6) & n.d. & n.d. & & & \\
\hline Rhubarb $(1,3)$ & n.d. -0.02 & n.d.-0.12 & 0.02 & 0.04 & n.d. \\
\hline Rhubarb, cooked $(1,3)$ & n.d.-0.03 & n.d. -0.05 & n.d. & 0.04 & n.d. \\
\hline Sharon (6) & n.d. & n.d. & & & 0.12 \\
\hline Strawberry $(1,6)$ & n.d.-0.02 & n.d.-0.03 & n.d. & n.d. & n.d. \\
\hline Watermelon $(1,3,4)$ & n.d. & n.d. & & & \\
\hline
\end{tabular}

Note: $\quad$ n.d. $=$ non-detectable or non-quantifiable.

Source: (1) Kuhnle et al. 2007; (2) Kuhnle et al. 2009b; (3) Liggins et al., 2000a; (4) Thompson et al. 2006; (5) Horn-Ross et al. 2000; (6) Mazur \& Adlercrutz 1998.

Table 6: Isoflavone content in berries, measured as $\mathrm{mg} / \mathrm{kg}$ fresh weight

$\begin{array}{llllll}\text { Type of food and processing } & \text { Genistein } & \text { Daidzein } & \text { Glycitein } & \text { Biochanin A } & \text { Formononetin } \\ \text { Blackcurrant }(2,3) & \text { n.d.-0.012 } & \text { n.d.-0.009 } & \text { n.d.-0.001 } & 0.068 & \text { n.d.-0.004 } \\ \text { Blueberry (2) } & 0.007 & 0.004 & 0.002 & 0.009 \\ \text { Cranberry (1,2) } & 0.031-0.061 & 0.006-0.007 & 0.001 & 0.003 \\ \text { Currants (1) } & 1.783 & 0.461 & & 0.006 \\ \text { Currants, dried (2) } & 0.1 & 0.022 & 0.002 & \text { n.d.-0.011 } \\ \text { Gooseberry (1) } & \text { n.d. } & \text { n.d. } & & \text { n.d.-0.002 } & \\ \text { Raspberry (1,2) } & \text { n.d.-0.048 } & \text { n.d.-0.032 } & \text { n.d. } & & \\ \text { Raspberry, canned (1) } & \text { n.d. } & & & \end{array}$

Note: n.d. = non-detectable or non-quantifiable.

Source: (1) Liggins et al., 2000a; (2) Thompson et al. 2006; (3) Clarke et al., $2013 a$. 
Table 7: Isoflavone content in berries, measured as $\mathrm{mg} / \mathrm{kg}$ dry weight

\begin{tabular}{|c|c|c|c|c|c|}
\hline Type of food and processing & Genistein & Daidzein & Glycitein & Biochanin A & Formononetin \\
\hline Blackcurrant $(1,2,3)$ & n.d. & n.d.-0.01 & n.d. & 0.08 & n.d. \\
\hline Blueberry (2) & n.d. & n.d. & & & \\
\hline Cloudberry (2) & n.d. & n.d. & & & \\
\hline Cranberry (2) & n.d. & n.d. & & & \\
\hline Gooseberry (1) & 0.02 & 0.02 & 0.03 & 0.02 & n.d. \\
\hline Lingonberry (2) & n.d. & n.d. & & & \\
\hline Raspberry $(1,2)$ & n.d. & n.d. & n.d. & 0.1 & n.d. \\
\hline Red currant (2) & n.d. & n.d. & & & \\
\hline
\end{tabular}

Note: $\quad$ n.d. $=$ non-detectable or non-quantifiable

Source: (1) Kuhnle et al. 2007; (2) Mazur \& Adlercrutz 1998; (3) Clarke et al., 2013 a.

As shown in Table 5 and Table 7, the isoflavone content in berries is generally low, possibly slightly higher than in fruits. The highest amounts were found in currants: $1.78 \mathrm{mg}$ total genistein and $0.46 \mathrm{mg}$ total daidzein per $\mathrm{kg} \mathrm{fw}$.

\subsubsection{Isoflavones in legumes}

The best dietary sources of isoflavones are the legumes. For humans, soybeans and soybean products are clearly most important, whereas for livestock also clover and alfalfa give a considerable contribution. Because isoflavones behave as phytoalexins (see subsection Stress conditions under section 3.1.1 Occurrence of isoflavones), a substantial variation in their levels in soybeans and other legumes is expected. In most other foodstuffs, it is the totals of daidzein, genistein, and glycitein that have been analysed, possibly in combination with the glycosylated conjugates daidzin, genistein, and glycitin. However, in legumes, and particularly in soybeans, twelve compounds have been searched, i.e. three glycosyl-malonylated compounds and three glycosylacetylated compounds in addition to the six compounds mentioned above. Table 8 and 9 reports the isoflavone levels in legumes when measured on a fw basis while Table 10 and 11 presents the levels in legumes when reported on a dw basis.

In the following subsections observations made on conventional soybean, genetically modified soybean, soybean sprouts, and other legumes than soybean are presented.

\section{Conventional soybeans}

Soybean is grown as a commercial crop in over 35 countries. It is now the most widely grown oilseed crop in the world, with approximately 266 million metric tons of harvested seed produced in 2012-2013, which represented $57 \%$ of world oilseed seed production that year.

Soybean seed contains a number of natural anti-nutritional components, including trypsin (proteinase) inhibitors, lectins, phytic acid, stachyose, and raffinose that prohibit the use of non-processed seeds (OECD, 2012b). Some of these antinutrients relate to their impact on human nutrition, whereas others relate to animal nutrition in general, including livestock. Adequate heating inactivates the protein antinutrients and therefore properly handled soybeans and processed products have been 
safely consumed as food and feed for a long time. Soy foods can be roughly classified into four major categories (Liu, 2004):

- Traditional soy foods: primarily made from whole soybean. The non-fermented traditional soy foods include soymilk, tofu, and soybean sprouts, whereas the fermented soy foods include soybean paste (miso), soybean sauce, natto, and tempeh.

- Soybean oil constitutes approximately $27 \%$ of the global consumption of vegetable oil and is the second largest source of vegetable oil worldwide (http://soystats.com/international-world-vegetable-oilconsumption). Refined, bleached, and deodorised soybean oil can be further processed and used in the manufacture of cooking oils, shortening, margarine, mayonnaise, salad dressings, and a wide variety of products that are either based entirely on fats and oils or contain fat or oil as a principal ingredient.

- Soybean protein products are made from defatted soybean flakes and include soybean flour, soybean protein concentrate, and soybean protein isolate.

Soybean flour has a protein content of approximately $50 \%$ and is used mainly as an ingredient in the bakery industry. Soybean protein concentrate has a protein content of approximately $70 \%$ and is used widely in the meat industry as a key ingredient of meat alternative products such as soybean burgers and "meatballs". Soybean protein isolate has a protein content of 90\% and possesses many functional properties such as gelation and emulsification. As a result, it can be used in a wide range of food applications, including soups, sauce bases, energy bars, nutritional beverages, infant formula, and dairy replacements.

- Dietary supplements due to soybeans richness in certain phytochemicals used as dietary supplements, in particular isoflavones and tocopherols.

Soybean is also an important feed source. Soybean meal is the most valuable component obtained from processing of the soybean, accounting for approximately $50-75 \%$ of its overall value. ${ }^{6}$ Soybean meal is produced by solvent (typically hexane) extraction of de-hulled soybean flakes. The process involves heating the spent flakes to evaporate the hexane and using steam to carry away hexane vapours. This process also provides toasting of the meal to inactivate anti-nutritional proteins that may reduce the digestibility and nutritional value of the meal. The meal is subsequently dried to about $13-14 \%$ moisture and ground to produce a uniform particle size. The finished meal from de-hulled soybean contains less than $1.5 \%(\mathrm{w} / \mathrm{w})$ crude fat and approximately $48 \%(\mathrm{w} / \mathrm{w})$ protein and is referred to as high protein meal (http://www.soymeal.org/FactSheets/processing3.pdf). Soybean meal is a premier supplemental protein source in livestock and poultry rations because of its nutrient composition, availability, and price. The green parts of the soybean plant, the soybean forage, can be used as hay or to produce silage.

\footnotetext{
${ }^{6}$ http://www.tanocapital.com/news/2011/11/29/tano-capital-research-soybeans-supply-and-demand.html
} 
Table 8: Isoflavone content (mg/kg fresh weight) in legumes. Soybean data are also shown in Table 9

\begin{tabular}{|c|c|c|c|c|c|}
\hline Types of food and processing & Genistein & Daidzein & Glycitein & Biochanin A & Formononetin \\
\hline Alfalfa sprouts $(2,3,4,5)$ & n.d. -1.176 & n.d. -1.517 & 0.006 & n.d. -0.669 & $3.4-51.7$ \\
\hline Chickpea (Cicer arietinum L.) (2) & 0.672 & n.d. & & 13.941 & 0.52 \\
\hline Clover sprouts $(2,5)$ & $0.709-3.5$ & n.d. -0.713 & & $4 \cdot 4-7 \cdot 512$ & $22.8-40.199$ \\
\hline Common bean (Phaseolus vulgaris), various types $(3,9)$ & $0.002-0.329$ & $0.002-0.191$ & n.q.-0.001 & & $0.002-0.029$ \\
\hline Common bean (Phaseolus vulgaris), various types (10)* & $0.010-0.015$ & $0.003-0.009$ & $0.011-0.013$ & $0.011-0.013$ & $0.006-0.017$ \\
\hline Lentil (Lens culinaris) $(3,5,6,7,10) * *$ & n.d. -0.188 & n.d. -0.104 & n.q. -0.014 & n.d. -0.013 & n.d. -0.196 \\
\hline Liquorice (Glycyrrhiza glabra) (3) & 0.216 & 0.223 & 0.011 & & 3.988 \\
\hline Lima beans (Phaseolus lunatus) $(3,5,7,9)$ & n.d. -0.847 & n.d. -0.89 & 0.001 & n.d. -3.7 & $0.001-5.5$ \\
\hline Mung bean (Vigna radiata) $(3,9,18)$ & n.d. -0.106 & n.d. -0.05 & 0.002 & & 0.005 \\
\hline Mung bean, sprouts $(2,3,9,18)$ & $1.352-4.241$ & $0.914-3.872$ & 0.012 & n.q. & $0.02-0.261$ \\
\hline Pea (Pisum sativum) $(1,9)$ & n.q. -0.144 & n.q. -0.041 & 0.01 & n.q. & n.q. \\
\hline Split pea, green (9) & 0.347 & 0.13 & & & \\
\hline Pea soup (3) & 0.003 & 0.001 & n.q. & & 0.001 \\
\hline Peanut (Arachis hypogaea) $(3,64,30)$ & $0.049-0.48$ & n.q.-0.077 & $0.004-0.1$ & 0.08 & $0.003-0.04$ \\
\hline Runner bean (Phaseolus coccineus) (9) & $1.183-2.26$ & $1.036-1.672$ & & & \\
\hline Soybean (Glycine $\max )(2,3,5,9,33,41,50,52,53,56,63)$ & $27-888$ & $22-677$ & $5.7-28.1$ & n.d. -3 & n.d. -0.077 \\
\hline Soybean flour $(18,36)$ & $427-960$ & $336.6-1,367.7$ & $32-167.4$ & & \\
\hline Soy milk $(2,3,15,26)$ & $18.522-91.8$ & $9.213-130.3$ & $1.695-4.41$ & n.d.-n.q. & n.d. -0.012 \\
\hline Soy milk, skin (15) & 835.9 & 469.5 & & & n.d. \\
\hline Soy nuts (3) & 362.64 & 283.51 & 38.948 & & 0.616 \\
\hline Soy protein powder (3) & 59.726 & 25.294 & 3.056 & & 0.163 \\
\hline Soy sauce $(2,3,15)$ & $0.599-14.5$ & $0.232-23.5$ & 0.054 & n.d. & n.d. -0.058 \\
\hline Soya infant formula (26) & 140.6 & 40.83 & 10.61 & n.d. & 0.07 \\
\hline Soybean protein, textured (3) & & $200-1,873$ & & & \\
\hline Soybean. germinated $(31) * * *$ & $873-1,563$ & $1,755-2,280$ & $115-347$ & & \\
\hline Soybean, sprout $(2,3,60)$ & $2.29-19.749$ & $4.45-24.244$ & n.d. -0.045 & n.d. & n.q. -1.867 \\
\hline Soybean-based tablets or capsules (20) & $0.003-0.007$ & $0.002-0.006$ & $0.000-0.001$ & & \\
\hline
\end{tabular}

Note: $\quad$ n.d. = non-detectable; n.q. = non-quantifiable; *In addition, $0.011-0.019 \mathrm{mg}$ daidzin, $0.052-1.104 \mathrm{mg}$ genistin, and $0.012-$ $0.019 \mathrm{mg}$ glycitin per $\mathrm{kg}$ fresh weight; **In addition, $0.005-0.016 \mathrm{mg}$ daidzein, $0.021-0.033$, and $0.006-0.018 \mathrm{mg}$ glycitin per $\mathrm{kg}$ fresh weight; $* * *$ In addition, 418-848 mg daidzin, 33-68 mg genistin, and 51-299 mg glycitin per $\mathrm{kg}$ fresh weight.

For references, see footnote to Table 11.

Table 9: Isoflavone content ( $\mathrm{mg} / \mathrm{kg}$ fresh weight) in soybeans and soybean products

\begin{tabular}{|c|c|c|c|c|c|}
\hline Isoflavone compound & $\begin{array}{l}\text { Soybean (Glycine } \max ) \\
(14,27,31,39,40,57,58,59)\end{array}$ & $\begin{array}{l}\text { Soybean flour } \\
(14,24,25)\end{array}$ & Soy granule (14) & $\begin{array}{l}\text { Soybean protein, } \\
\text { textured (13) }\end{array}$ & Soybean, green (14) \\
\hline Daidzein & n.q. $-1,223$ & n.d. -210 & 12 & 13 & 10 \\
\hline Genistein & $2-1,489$ & n.d. -62 & 27 & 31 & 16 \\
\hline Glycitein & n.q. -204 & n.d. -120 & 22 & 26 & 18 \\
\hline Daidzin & $12.5^{-1,331}$ & $147-1,202$ & 727 & 660 & 451 \\
\hline Genistin & $28.4-2,340$ & $407-1,448$ & 870 & 524 & 430 \\
\hline Glycitin & $30-478$ & $41-243$ & 132 & 150 & 48 \\
\hline Malonyldaidzin & $198-2,200$ & 261 & 106 & 197 & 515 \\
\hline Malonylgenistin & $399-3,981$ & 1,023 & 193 & 95 & 851 \\
\hline Malonylglycitin & $39-650$ & 57 & 60 & 63 & 57 \\
\hline Acetyldaidzin & n.q. -370 & n.q. & 72 & 334 & n.q. \\
\hline Acetylgenistin & n.q. -31 & 1 & 135 & 193 & 2 \\
\hline Acetylglycitin & n.q.-9o & 32 & 48 & 93 & n.d. \\
\hline
\end{tabular}

Note: For references, see footnote to Table 11. 
Table 10: Isoflavone content (mg/kg dry weight) in legumes. Soybean data are also shown in Table 11

\begin{tabular}{|c|c|c|c|c|c|}
\hline Type of food and processing & Genistein & Daidzein & Glycitein & Biochanin A & Formononetin \\
\hline Adzuki bean seed (Vigna angularis) (1) & 21.2 & 4.6 & & & \\
\hline Alfalfa, sprouting ( 7 ) & 0.115 & 0.037 & & n.d. & n.d. \\
\hline Broad bean $(3,5,7,8,9)$ & n.d.-o.01 & n.d.-0.31 & n.q. -8 & n.d.-n.q. & n.d.-0.39 \\
\hline Broad bean (Vicia fava) (1) & 19.9 & 5 & & & \\
\hline Chickpea $(5,6,7,10,11)^{*}$ & n.d. $-0.766(2.14)$ & n.d. $-0.475(1.92)$ & $0.645-0.852$ & $0.706-30.8$ & n.d. -10 \\
\hline Clover, flower (1) & 110 & 73.6 & & & \\
\hline Clover, seed (6) & 3.23 & 1.78 & & 3.81 & 12.7 \\
\hline $\begin{array}{l}\text { Common bean (Phaseolus vulgaris), } \\
\text { various types }(1,3,4,5,6,7,12,17)\end{array}$ & n.d. -45.1 & n.d. -129.1 & & n.d. -6 & n.d. -8.2 \\
\hline Haricots verts (green beans) $(5,7,8)$ & n.d. -4.08 & n.d.-1.24 & 0.2 & n.q. -0.16 & n.d. -1.5 \\
\hline Cowpea (Vigna unguiculata) $(6,7)$ & $0.12-0.56$ & $0.21-0.3$ & & n.d. & n.d. \\
\hline $\begin{array}{l}\text { Black-eyed bean/pea (Vigna unguiculata } \\
\text { subs. ungiuculata) }(1,5,7)\end{array}$ & n.d. -23.3 & n.d. -0.3 & & $0.077-17.3$ & n.d. -0.055 \\
\hline Kudzu (Pueraria lobata), leaf ( 7 ) & 25.2 & 3.75 & & 12.4 & 0.87 \\
\hline Kudzu (Japanese arrow root), root (1.6) & $126-316.9$ & $949.8-1850$ & & 14 & 70.9 \\
\hline Kudzu (Japanese arrow root), stem (1) & $39 \cdot 3$ & 30.9 & & & \\
\hline Lima bean seed (Phaseolus lunatus) $(1,5)$ & n.d. -40.1 & n.d. -0.4 & & n.d. & n.q. \\
\hline $\begin{array}{l}\text { Lentil (Lens culinaris), various varieties } \\
(1,7,10)\end{array}$ & $0.01-0.071,(25)$ & $0.02-0.033(5.2)$ & & 0.071 & 0.075 \\
\hline Lupin (Lupinus mutabilis or L. albus) $(1,8)$ & $24.2-59.2$ & n.d. -46.6 & & n.d. & 0.231 \\
\hline Lupine, leaf (1) & $86.7-101$ & n.d. -38.9 & & & \\
\hline Mung bean (Vigna radiata) $(1,5,7)$ & n.d. -21.8 & n.d. -0.097 & & n.d. -0.141 & $0.075-6.1$ \\
\hline Mung bean, sprouts (5) & n.d. & n.d. & & n.d. & n.q. \\
\hline Black gram (Vigna mungo) $(6,7)$ & n.q. -0.603 & $0.07-0.36$ & & n.d.-0.811 & n.d. -0.02 \\
\hline Pea (Pisum sativum). $(5,6,7,8)$ & n.d.-0.23 & n.d. -0.113 & $0.03-8.6$ & n.d. -0.044 & n.d. -0.049 \\
\hline Green split pea $(4,5,7)$ & n.d. -0.02 & $0.02-72.6$ & n.q. & n.d. & n.d.-n.q. \\
\hline Peanut (Arachis hypogaea) $(6,7,16)$ & $0.13-0.826$ & $0.497-1.3$ & & $0.065-0.45$ & 0.068 \\
\hline Peanut, defatted (16) & $0.13-2.27$ & $1.25-10.63$ & & $0.37-1.37$ & \\
\hline Pigeon pea (Red gram) (Cajanus cajan) $(6,7)$ & $1.9-7.37$ & $0.12-0.272$ & & $0.104^{-2.19}$ & $0.051-0.261$ \\
\hline $\begin{array}{l}\text { Red clover (Trifolium pratense), greens } \\
(1,7,19)\end{array}$ & $26-680$ & $5-330$ & & $700-3,300$ & $223-5,900$ \\
\hline $\begin{array}{l}\text { Soybean }(4,5,6,7,15,18,22,38,45,46,47, \\
49,51,54,55,61)\end{array}$ & $267-1,950$ & $105-2,550$ & $37-1,300$ & n.d. -0.147 & n.d.-1.21 \\
\hline Soybean flour $(5,7,9,22)$ & $503-1,123$ & $655^{-1,037}$ & & n.d-0.744 & n.d. -0.302 \\
\hline Soybean, germ (47) & $993-1,618$ & $4,730-12,277$ & $2848-6850$ & & \\
\hline Soybean sprout (7) & 69.31 & 12.92 & 0.12 & n.q. & n.q. \\
\hline Soybean sprout $(4,15,22)$ & $517-1,458$ & $165-1,334$ & & & n.d. \\
\hline Tepary bean (Phaseolus acutifolius) (1) & 0.14 & n.q. & n.q. & 0.02 & n.d. \\
\hline
\end{tabular}

Note: $\quad$ n.d. = non-detectable; n.q. = non-quantifiable; *In addition, $0.011-0.018 \mathrm{mg}$ daidzin, $0.043-0.057 \mathrm{mg}$ genistin, and $0.193^{-0.270 ~ m g ~ g l y c i t i n ~ p e r ~ k g ~ d r y ~ w e i g h t ~}$

For references, see footnote to Table 11. 
Table 11: Isoflavone content ( $\mathrm{mg} / \mathrm{kg}$ dry weight) in soybeans and soybean products

\begin{tabular}{|c|c|c|c|c|c|}
\hline Isoflavone compound & $\begin{array}{l}\text { Soybean }(1,4,12,13,21,28,32, \\
34,35,37,42,43,44,48,51,62)\end{array}$ & $\begin{array}{l}\text { Soybean, } \\
\text { defatted (29) }\end{array}$ & $\begin{array}{l}\text { Soybean, } \\
\text { germinated (48) }\end{array}$ & $\begin{array}{l}\text { Soybean, } \\
\text { germ (13) }\end{array}$ & $\begin{array}{l}\text { Soy milk } \\
\text { (13) }\end{array}$ \\
\hline Daidzein & n.q. -199.2 & 76.9 & $10-29$ & 303 & 18 \\
\hline Genistein & n.q. -108.4 & 154 & $7-24$ & 114 & 19 \\
\hline Glycitein & n.d. -28.6 & 23 & $11-17$ & 684 & 10 \\
\hline Daidzin & $19-3,404$ & 147 & $67-106$ & 5,208 & 410 \\
\hline Genistin & n.d. $-1,981$ & 231 & $150-186$ & 1,664 & 710 \\
\hline Glycitin & $6.7-335.7$ & 60.2 & $82-102$ & 6,505 & 65 \\
\hline Malonyldaidzin & $87-3,630$ & 576 & $407-677$ & 3,466 & 690 \\
\hline Malonylgenistin & $26-2,490$ & 730 & $1,076-1,308$ & 857 & 871 \\
\hline Malonylglycitin & n.d. -617 & 226 & $158-189$ & 3,313 & 39 \\
\hline Acetyldaidzin & n.d. -468 & n.d. & $90-111$ & 10,830 & 22 \\
\hline Acetylgenistin & n.q. -148 & n.d. & $10-25$ & 2,616 & 829 \\
\hline Acetylglycitin & n.d. -134 & n.d. & $39-47$ & 3,952 & 29 \\
\hline
\end{tabular}

Note: $\quad$ n.d. $=$ non-detectable; $n$.q. $=$ non-quantifiable.

(1) Kaufman et al., 1997; (2) Horn-Ross et al., 2000; (3) Thompson et al., 2006; (4) Knuckles et al., 1976; (5) Franke et al., 1994; (6) Mazur \& Adlercreutz, 1998; (7) Mazur et al., 1998b; (8) Kuhnle et al., 2007; (9) Liggins et al., 200ob; (10) Konar et al., 2012; (11) Wu et al., 2012; (12) de Lima et al., 2014; (13) Song et al., 1998; (14) Wang \& Murhphy, 1994a; (15) Wang et al., 1990; (16) Chukwumah et al., 2007a; (17) Diaz-Batalla et al., 2006; (18) Müllner \& Sontag, 1999; (19) Booth et al., 2006; (20) Penalvo et al., 2004; (21) Romani et al., 2003; (22) Devi et al., 2009; (23) Eldridge, 1982; (24) Coward et al., 1993; (25) Klump et al., 2001; (26) Kuhnle et al., 2008a; (27) Eldrige \& Kwolek, 1983; (28) Tepavcevic et al., 2010; (29) Paucar-Menacho et al., 2010; (30) Liggins et al., 2000a; (31) Huang et al., 2014; (32) Mo et al., 2013; (33) Sakakibara et al., 2003; (34) Xu \& Chang, 2008c; (35) Mebrahtu et al., 2004; (36) Whent et al., 2011; (37) Ribeiro et al., 2007; (38) Morrison et al., 2008; (39) Lee et al., 2004; (40) Carrao-Panizzi \& Kitamura, 1995; (41) Primomo et al., 2005; (42) Sakthivelv et al., 2008; (43) Simonne et al., 2000; (44) Tsukamoto et al., 1995; (45) Harrigan et al., 2010b; (46) Malencic et al., 2012; (47) Hubert et al., 2005; (48) Zhu et al., 2005; (49) Bermann et al., 2010; (50) Bermann et al., 2011; (51) Fast et al., 2015; (52) Goda et al., 2002; (53) Harrigan et al., 2010a; (54) Lepping et al., 2013; (55) Lundry et al., 2008; (56) Zhou et al., 1998; (57) Cvejic et al., 2009; (58) Phommalth et al., 2008; (59) Riedl et al., 2007; (60) Lee et al., 2013; (61) Leuner et al., 2012; (62) Xu and Chang, 2008a; (63) Xu \& Chang, 2008b; (64) Kuhnle et al., 2008b.

Soybeans are a good source of protein, producing more protein per unit of land than most major plant or animal sources used today. Soybeans have a high content of essential amino acids, particularly lysine, leucine, and isoleucine (Fehily, 2003). The three major soybean commodity products are beans, oil, and meal (or cake). Whole processed soybeans are used to produce baked soybeans, roasted soybeans, full fat soy flour, and the traditional soy foods (miso, soy milk, soy sauce, and tofu). However, the primary use of soybean is as a heat-processed (toasted) defatted (TD) meal for protein supplementation of animal feeds. Soybean oil is the main food ingredient derived from soybean. Soybean oil is extensively used in products such as cooking and salad oils, salad dressings, shortening, and oleo margarine. Lecithin, a phosphatide removed from crude soybean oil, is used as a natural emulsifier, lubricant, and stabilising agent. Glycerol, fatty acids, and sterols are also derived from soybean oil (OECD, 2012b).

The food industry is increasingly using products derived from soybeans as ingredients, the reason being they can influence the physical structure, stability, and texture of foods. Defatted, de-hulled soybean flakes, minimally heat treated to retain 
solubility, are employed as a starting material for a wide variety of soybean food products (Snyder \& Wilson, 2003). Soybean protein products, manufactured from defatted soybean flakes, are added to many meats, dairy, bakery, and cereal products. Soybean protein isolate is the protein source for soybean-based infant formula. Soybean protein concentrates are incorporated in some meat products as an extender but also in a textured form to simulate meat. Soybean flour products, either relecithinated or refatted, manufactured from defatted soybean flakes, are added to many baked goods. Soybean hulls are a high natural source of dietary fibre that can be processed into fibre bran breads, cereals, and snacks (OECD, 2012b).

The data in Tables 8 and 11 demonstrate that soybean is the food crop containing the highest total level of isoflavones. As shown in these Tables, there are close to fifty studies that have measured the isoflavone profile in soybeans. Twenty of these have reported the data in $\mathrm{mg} / \mathrm{kg} \mathrm{fw}$ (Table 8 and 9) and twenty-eight in $\mathrm{mg} / \mathrm{kg} \mathrm{dw}$ (Table 10 and 11). In these Tables, the reports have been divided into those that used a harsh extraction/hydrolysis method and therefore were only able to identify the aglycones (and possibly the simple isoflavone glucosides) with precision (Table 8 and 10) and those that used methods that were somewhat successful in separating individual conjugates from the aglycones (Tables 9 and 11). Independent of which extraction method and chemical analytical methodology were used to identify and quantify the compounds and whether levels have been reported in $f w$ or in $d w$, there are huge variations in the levels reported by the various research groups. These variations are partly due not only to the genetic differences between the studied soybean materials and the different environments where they have been grown but also to the different methods that have been used for the chemical analysis. The variation in isoflavone content due to the biology was reviewed in subsection Factors influencing isoflavone content under section 3.1.1 Occurrence of isoflavones.

Kudou et al. (1991) studied the accumulation of isoflavone constituents in soybean seeds during development. Malonylgenistin and genistin content increased during the late development of the beans, whereas malonyldaidzin and daidzin accumulated throughout the whole period. Small quantities of malonylglycitin and glycitin were also detected. In mature seeds, malonylgenistin and malonyldaidzin comprised $66 \%$ of the total isoflavone content. The three acetylated forms only made up about $5 \%$ of total isoflavones.

Tepavčevič et al. (2010) analysed and compared the isoflavone content of 20 soybean varieties of maturity classes o-Il (short-season northern-type soybean genotypes) of different origin (five each from Serbia, USA, Russia, and China) to determine the influence of genotype on isoflavone composition in soybean. Twelve isoflavones were measured in every soybean variety. The amount of total isoflavones varied threefold (between 1,450 and 4,590 $\mu \mathrm{g} / \mathrm{g}$ of soybean), with an average value of $3,250 \mu \mathrm{g} / \mathrm{g}$. A similar magnitude of variation was reported in thirteen soybean varieties from the USA, Brazil, Argentina, Canada, and China (Zhang et al., 2012) and in thirteen varieties studied by Genovese et al. (2005). Other investigators have reported an even larger variation. Thus, Lee et al. (2002) reported a fivefold variation in 46 soybean varieties cultivated in Korea and Carrao-Panizzi and Kitamura (1995) a 3-8-fold variation in 22 conventional Brazilian soybean cultivars, with highest levels in an insect-resistant 
cultivar. When the isoflavone profile of the various soybean varieties in the study of Tepavčevič et al. (2010) was compared, the concentration of acetyl-genistein was lowest in all analysed samples (average $11.9 \mu \mathrm{g} / \mathrm{g}$ ), whereas the concentration of malonyldaidzin was highest (average $2062 \mu \mathrm{g} / \mathrm{g})$. In general, malonyl-genistin $(1840 \mu \mathrm{g} / \mathrm{g})$, genistin $(561 \mu \mathrm{g} / \mathrm{g})$, and daidzin $(461 \mu \mathrm{g} / \mathrm{g})$ followed malonyl-daidzin in a decreasing order. The most common isoflavone, expressed as aglycone, was daidzein ( $47.2 \%$ of all isoflavones), followed by genistein (40.2\%) and glycitein (12.6\%). The entire profile of isoflavones in soybeans was also studied by Wang and Murphy in 1994. The latter investigators measured the amount of twelve isoflavones in eight American and three Japanese soybean varieties cultivated from 1989 to 1991 . The total isoflavone content ranged from 1176 to $3309 \mathrm{mg} / \mathrm{kg} \mathrm{dw}$, with the major components in declining order being 6"-O-malonylgenistin, genistin, 6"-O-malonyldaidzin, and daidzin (Wang \& Murphy, 1994a).

$\mathrm{XU}$ and Chang (2008c) characterised the phenolic substances in thirty varieties of soybean grown in the North Dakota - Minnesota region in 2005 or 2006. This region is one of the highest latitude geographical zones in the USA for growing warm season legume soybeans. Twenty-seven of the varieties were normal lipoxygenase soybean samples and three were lipoxygenase-free soybeans. Ten of the twelve individual isoflavones (three 7-O-glucosides, three 6"'-O-malonyl- $\beta$-glucosides, three 6 "' $O$ acetyl- $\beta$-glucosides, and three aglycones) were found in all soybean varieties. Whereas acetylgenistin was detected in most soybean cultivars, glycitein was detected in only three cultivars. The highest proportion at more than $75 \%$ of the total was 6 " $-O$-malonyl$\beta$-glucosides (sum of the three), followed by the $7-O$-glucosides (sum of the three) at $20 \%$. 6"-O-acetyl- $\beta$-glucosides and aglycones existed in only very small proportions. Summing up the various isoflavones showed the subtotal of genistein to account for $69.2 \%$, daidzein for $24.6 \%$, and glycitein for $6.3 \%$. Quinhone Junior and Ida (2015) limited their studies to one Brazilian soybean variety (BRS 284) and observed that the seeds contained $26.0 \% \beta$-glucosides, $72.9 \%$ malonylglucosides, and $1.2 \%$ aglycones.

Apparently, both genetics and the environment can influence the proportion of the subtotal of the three primary isoflavones. In contrast to $\mathrm{XU}$ and Chang (2008c), Zhang et al. (2012) reported that total daidzein was usually the dominant primary isoflavone. However, according to Manach et al. (2004), the total of the three main isoflavones found in soybean seed (genistein, daidzein, and glycitein) is generally in the concentration ratio 1:1:0.2.

Similar proportions of total isoflavones, with malonyl-isoflavones being the major one, as reported by $\mathrm{Xu}$ and Chang (2008c) and Quinhone Junior and Ida (2015) (i.e. $75 \%$ and $72.9 \%$, respectively) have been found by Ribeiro et al. (2007). Based on studies of 18 soybean cultivars of different maturity groups grown in Brazil, these investigators concluded that malonyldaidzin and malonylgenistin are the predominant isoflavone forms in all cultivars, corresponding to $67 \%$ of total isoflavones. The $\beta$-glucoside form corresponded to $31 \%$ of the total isoflavones and aglycone to $2 \%$. The acetylated form was not detected by these investigators.

Sakthivelu et al. (2008) analysed the isoflavone profile and the levels of individual compounds in four soybean varieties grown in India and seven cultivars grown in 
Bulgaria. The range in total isoflavones was $558-1049 \mathrm{mg} / \mathrm{kg} \mathrm{dw}$ in the Indian cultivars and $628-1717 \mathrm{mg} / \mathrm{kg}$ in the Bulgarian cultivars. A good correlation with total isoflavone level was found for the malonylglucosides of each individual aglycone, as well as for total genistein and total daidzein. In contrast, acetylglucoside and aglycone levels did not significantly correlate with total isoflavone content.

From the studies analysing all twelve isoflavone constituents in soybean and referred to above, it is evident that the large majority of the compounds occur as conjugated forms in the soybean. Genovese et al. (2005) reported that 90-95\% of the total isoflavones were conjugated forms. They also reported that total genistein varied between 39 and $57 \%$, total daidzein between 34 and $47 \%$, and total glycitein between 8 and $17 \%$.

Flavonoids have been identified to contribute to an undesirable taste of soybean foods, sometimes described as dry mouth feel (Huang et al., 1981, 1982; Kudou et al., 1991; Okubo et al., 1992). Daidzin and genistin are hydrolysed by $\beta$-glucosidase to daidzein and genistein, respectively, which results in an increase of the objectionable flavour in soymilk (Matsuura et al., 1989; Okubo et al., 1992). Because the conversion of the isoflavone conjugates to the aglycons is increased at higher temperatures, it is favourable not to use high temperatures during soy milk manufacturing.

\section{Genetically modified soybeans}

At the time when this report was drafted (October-November 2015), 24 applications to market genetically modified soybeans as food and feed for import and processing within the European Union had been validated by the European Food Safety Authority. These applications are summarised in Table 12.

As a starting point in the risk assessment of new genetically modified (GM) plants, applicants compare the chemical composition of the newly developed GM plant with the composition of a control plant having very similar genetic background (preferably isogenic) to the GM plant. The Task Force for the Safety of Novel Foods and Feeds under the Organisation for Economic Co-operation and Development (OECD) has developed Consensus Documents suggesting which plant tissues and constituents should be analysed for new varieties. Such a document was developed for soybeans in 2001 and was revised in 2012 (OECD, 2012b). The isoflavones (total daidzein, total genistein, and total glycitein) are included in the list of compounds to be analysed in soybean seeds.

The compositional data on seeds of GM soybeans have been derived from materials harvested from a set of carefully controlled replicated field trials having a randomised complete block design. In these field trials, the GM soybean variety has been grown side-by-side to the comparator having a similar genetic background, and frequently to a set of commercial non-GM soybean reference varieties. Table 13 shows the results of the comparison of the level of isoflavones in harvested materials of the various GM soybeans and their conventional counterparts (control materials), noting whether the levels are comparable (NS = non-significant) or have been increased or reduced in the GM variety. In the latter case, the relative magnitude of the difference is given within brackets. 
Table 12: Summary of applications to market genetically modified (GM) soybean within the European Community and information on whether compositional data on isoflavones of the GM soybean has been published

\begin{tabular}{|c|c|c|c|}
\hline Variety & Applicant & Traits & $\begin{array}{l}\text { Reference(s) to } \\
\text { published isoflavone } \\
\text { data }\end{array}$ \\
\hline $40-3-2$ & Monsanto & Glyphosate tolerance & $\begin{array}{l}\text { Padgette et al., } 1996 \\
\text { Taylor et al., } 1999 \\
\text { McCann et al., } 2005 \\
\text { Harrigan et al., } 2007 \\
\text { Zhou et al., 2011a }\end{array}$ \\
\hline MON 89788 & Monsanto & Glyphosate tolerance & Lundry et al., 2008 \\
\hline MON 87701 & Monsanto & Lepidopteran resistance & Berman et al., 2010 \\
\hline MON $87701 \times$ MON89788 & Monsanto & Glyphosate tolerance and lepidopteran resistance & Berman et al., 2011 \\
\hline MON 87705 & Monsanto & Altered fatty acid profile and glyphosate tolerance & Data not published \\
\hline MON $87705 \times$ MON 89788 & Monsanto & Altered fatty acid profile and glyphosate tolerance & Data not published \\
\hline MON 87708 & Monsanto & Dicamba tolerance & Data not published \\
\hline MON $87708 \times$ MON 89788 & Monsanto & Dicamba and glyphosate tolerance & Data not published \\
\hline MON 87769 & Monsanto & Stearidonic acid rich fatty acid profile & Data not published \\
\hline MON $87769 \times$ MON 89788 & Monsanto & Steariconic acid rich fatty acid profile and glyphosate tolerance & Data not published \\
\hline MON 87751 & Monsanto & Resistance to lepidopteran insects & Data not published \\
\hline A $2704-12$ & Bayer & Glufosinate tolerance & Data not published \\
\hline A $5547-127$ & Bayer & Glufosinate tolerance & Data not published \\
\hline $\mathrm{FG}_{72}$ & Bayer & Isoxaflutole and glyphosate tolerance & Data not published \\
\hline $\mathrm{A}_{5547-127 \times \mathrm{FG}_{72}}$ & Bayer & Isoxaflutole, glyphosate, and glufosinate tolerance & Data not published \\
\hline 356043 & Pioneer & Tolerance to glyphosate, glyphosate ammonium, and ALS-inhibiting herbicides & Data not published \\
\hline 305423 & Pioneer & Altered fatty acid profile and tolerance to ALS-inhibiting herbicides & Data not published \\
\hline $305423 \times 40-3-2$ & Pioneer & Altered fatty acid profile and tolerance to glyphosate and ALS-inhibiting herbicides & Data not published \\
\hline DAS-68416-4 & Dow & $2,4^{-D}$ and glufosinate ammonium tolerance & Herman et al., 2011 \\
\hline DAS-44406-6 & Dow & Tolerance to 2,4-D, glyphosate, and glufosinate & Lepping et al., 2013 \\
\hline DAS-81419-2 & Dow & Resistant to several lepidopteran pests* & Fast et al., 2015 \\
\hline DAS-68416-4 × MON 89788 & Dow & Tolerance to $2,4-D$, glufosinate ammonium, and glyphosate & Data not published \\
\hline BPS-CV127-9 & BASF & Imidazolinone herbicide tolerance & Data not published \\
\hline SYHToH2 & Syngenta & Mesotrione and glufosinate tolerance & Data not published \\
\hline
\end{tabular}

Note: *Glufosinate tolerance was a selectable marker gene. 
As seen in Table 12, several of the GM soybeans developed have been made tolerant to one or more herbicides (40-3-2, MON 89788, MON 87705, MON 87708, A2704-12, A5547-127, FG72, 356043, 305423, DAS-68416-4, DAS-44406-6, BPS-CV127-9, $\mathrm{SYHToH} 2$, MON $87701 \times \mathrm{MON} 89788$, MON $87705 \times \mathrm{MON} 89788$, MON $87708 \times \mathrm{MON}$ $89788, A_{5547-127} \times \mathrm{FG}_{72}, 305423 \times 40-3-2$, DAS-68516-4 $\times$ MON 89788) by introducing into the soybean by genetic modification specific genes responsible for the trait. When the trait in the GM soybean aims at giving the crop tolerance to a specific herbicide, the field trials usually contain both plots of the GM soybean not sprayed with the target herbicide and plots sprayed with the target herbicide. The target herbicide spraying regimen is frequently given on top of the field management required at the particular field trial site. The management includes soil preparation, fertilization, and required pesticide treatment applied equally to all soybean plots. For GM events of this type, Table 13 presents data on the comparison target herbicidetreated GM to control and on the untreated GM to control, when available. In none of the cases, has the control been treated with the target herbicide in the same way as the GM soybean, as that would kill the plant. 


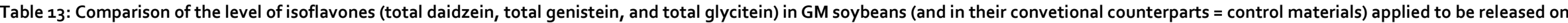

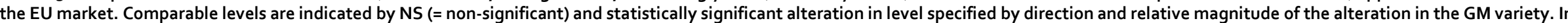

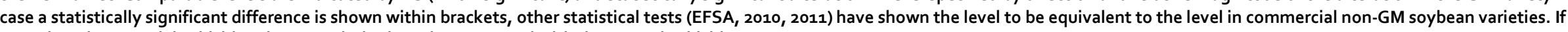
not otherwise stated, herbicide tolerant varieties have been sprayed with the target herbicide

\begin{tabular}{|c|c|c|c|c|c|c|c|}
\hline GM event & Control (maturity class) & Location & Year & Total Daidzein & Total Genistein & Total Glycitein & Reference \\
\hline $40-3-2^{*}$ & $A_{5403}(V)$ & 9 sites in the USA & 1992 & NS & NS & - & Padgette et al., 1996 \\
\hline $40-3-2$ & $\mathrm{~A}_{5403}(\mathrm{~V})$ & 4 sites in the USA & 1993 & NS & NS & - & Taylor et al., 1999 \\
\hline $40-3-2$ & Dekabig $(l+)$ & 5 sites in Romania & 2005 & NS & $\downarrow_{4 \%} \%$ & NS & Harrigan et al., 2007 \\
\hline \multirow{3}{*}{ 40-3-2 (25 genetic backgrounds) } & \multirow{3}{*}{$\begin{array}{l}\text { Various ( } 25 \text { non-matched } \\
\text { genetic backgrounds) }\end{array}$} & \multirow[t]{3}{*}{ Various } & 2000 & NS & NS & $\uparrow_{4 \%}$ & \multirow[t]{3}{*}{ McCann et al., 2005} \\
\hline & & & 2001 & NS & NS & NS & \\
\hline & & & 2002 & NS & NS & NS & \\
\hline $40-3-2$ (11-25 genetic backgrounds) & $\begin{array}{l}\text { Various (11-25 non-matched } \\
\text { genetic backgrounds) }\end{array}$ & Various & $2000-2009$ & NS & NS & NS & Zhou et al., $2011 a$ \\
\hline \multirow[t]{2}{*}{ MON 89788} & \multirow[t]{2}{*}{ A3244 (III) } & 5 sites in the USA & 2005 & $\downarrow_{7 \%}$ & NS & $\downarrow_{11 \%}$ & \multirow[t]{2}{*}{ Lundry et al., 2008} \\
\hline & & 5 sites in Argentina & $2004 / 2005$ & NS & NS & NS & \\
\hline MON 89788 & Monsoy 8329 (VIII) & 4 sites in northern Brazil & $2007 / 2008$ & NS & NS & NS & Berman et al., 2010 \\
\hline MON 89788 & A5547 (V) & 2 sites in southern Brazil & $2007 / 2008$ & $\uparrow_{15 \%}$ & NS & NS & Berman et al., 2010 \\
\hline \multirow[t]{2}{*}{ MON 87701} & \multirow[t]{2}{*}{ A5547 (V) } & 5 sites in the USA & 2007 & $\uparrow_{10 \%}$ & NS & NS & \multirow[t]{2}{*}{ Berman et al., 2009} \\
\hline & & 5 sites in Argentina & $2007 / 2008$ & NS & NS & NS & \\
\hline MON 87701 & $A_{5547}(\mathrm{~V})$ & 2 sites in southern Brazil & $2007 / 2008$ & NS & NS & NS & Berman et al., 2010 \\
\hline MON 87701 & Monsoy 8329 (VIII) & 4 sites in northern Brazil & $2007 / 2008$ & NS & NS & NS & Berman et al., 2010 \\
\hline \multirow[t]{4}{*}{ MON 87701 × MON 89788* } & \multirow[t]{4}{*}{$A_{5547}(V)$} & 5 sites in the USA & 2007 & $\uparrow 26 \%$ & $\uparrow_{20 \%}$ & NS & \multirow[t]{4}{*}{ Berman et al., 2011} \\
\hline & & 5 sites in Argentina & $2007 / 2008$ & $\uparrow_{13 \%} \%$ & $\uparrow_{10 \%}$ & NS & \\
\hline & & 2 sites in southern Brazil & $2007 / 2008$ & $\uparrow 21 \%$ & $\uparrow_{14 \%} \%$ & NS & \\
\hline & & 2 sites in southern Brazil & $2008 / 2009$ & $\uparrow_{18 \%}$ & $\uparrow_{10 \%}$ & NS & \\
\hline \multirow[t]{2}{*}{ MON 87701 × MON 89788* } & \multirow[t]{2}{*}{ Monsoy (VIII) } & 4 sites in northern Brazil & $2007 / 2008$ & NS & NS & NS & \multirow[t]{2}{*}{ Berman et al., 2011} \\
\hline & & 4 sites in northern Brazil & $2008 / 2009$ & NS & NS & NS & \\
\hline \multirow[t]{2}{*}{ MON 87705} & \multirow[t]{2}{*}{ A3525 (III) } & 5 sites in Chile & $2007 / 2008$ & NS & NS & NS & \multirow[t]{2}{*}{ EFSA-GMO-NL-2010-78 } \\
\hline & & 4 sites in the USA & 2008 & NS & NS & NS & \\
\hline MON $87705 \times$ MON 89788 (untreated) & A3525 (III) & 8 sites in the USA & 2009 & NS & $(\uparrow 5 \%)$ & NS & EFSA-GMO-NL-2011-100 \\
\hline
\end{tabular}




\begin{tabular}{|c|c|c|c|c|c|c|c|}
\hline GM event & Control (maturity class) & Location & Year & Total Daidzein & Total Genistein & Total Glycitein & Reference \\
\hline MON $87705 \times$ MON 89788 (glyphosate-treated) & A 3525 (III) & 8 sites in the USA & 2009 & NS & $(\uparrow 5 \%)$ & NS & EFSA-GMO-NL-2011-100 \\
\hline MON 87708 (untreated) & A3525 (III) & 8 sites in the USA & 2009 & NS & NS & NS & EFSA-GMO-NL-2011-93 \\
\hline MON 87708 (dicamba-treated) & A3525 (III) & 8 sites in the USA & 2009 & NS & $(\uparrow 6 \%)$ & $(\uparrow 7 \%)$ & EFSA-GMO-NL-2011-93 \\
\hline MON $87708 \times$ MON 89788 (untreated) & A3525 (III) & 8 sites in the USA & 2009 & $\uparrow_{4 \%} \%$ & $(\uparrow 13 \%)$ & NS & EFSA-GMO-NL-2012-108 \\
\hline $\begin{array}{l}\text { MON } 87708 \times \text { MON } 89788 \text { (dicamba and } \\
\text { glyphosate treated) }\end{array}$ & A 3525 (III) & 8 sites in the USA & 2009 & NS & $\left(\uparrow_{10 \%)}\right.$ & NS & EFSA-GMO-NL-2012-108 \\
\hline MON 87769 & A3525 (III) & $\begin{array}{l}5 \text { sites in the USA } \\
5 \text { sites in the USA }\end{array}$ & $\begin{array}{l}2006 \\
2007\end{array}$ & $\begin{array}{l}\downarrow_{34 \%} \% \\
\downarrow_{30 \%}\end{array}$ & $\begin{array}{l}\downarrow_{35 \%} \\
\downarrow_{31 \%}\end{array}$ & $\begin{array}{l}\downarrow_{19 \%} \\
\text { NS }\end{array}$ & EFSA-GMO-UK-2009-76 \\
\hline MON 87769 × MON 89788* & A3525 (III) & 5 sites in the USA & 2007 & $\downarrow 29 \%$ & $\downarrow 29 \%$ & NS & EFSA-GMO-NL-2010-85 \\
\hline MON 87751 & A3555 (III) & 8 sites in the USA & 2012 & NS & NS & NS & EFSA-GMO-NL-2014-121 \\
\hline A2704-12 (untreated) & A2704 (II) & $\begin{array}{l}4 \text { sites in the USA + } 5 \text { sites in the } \\
\text { USA }\end{array}$ & $1999+2000$ & NS & $\downarrow_{13 \%}$ & NS & EFSA-GMO-NL-2005-18 \\
\hline A2704-12 (glufosinate treated) & $\mathrm{A}_{2704}$ (II) & $\begin{array}{l}4 \text { sites in the USA + } 5 \text { sites in the } \\
\text { USA }\end{array}$ & $1999+2000$ & $\downarrow_{13} \%$ & $\downarrow_{13 \%}$ & NS & EFSA-GMO-NL-2005-18 \\
\hline A5547-127 (untreated) & A5547 (V) & $\begin{array}{l}4 \text { sites in the USA + } 5 \text { sites in the } \\
\text { USA + } 7 \text { sites in the USA }\end{array}$ & $1999+2000+2006$ & $\begin{array}{l}5 \text { of } 16 \text { trials } \\
\text { were significant }\end{array}$ & $\begin{array}{l}2 \text { of } 16 \text { trials were } \\
\text { significant }\end{array}$ & $\begin{array}{l}5 \text { of } 16 \text { trials } \\
\text { were significant }\end{array}$ & EFSA-GMO-NL-2008-52 \\
\hline A5547-127 (glufosinate-treated) & A5547 (V) & $\begin{array}{l}4 \text { sites in the USA + } 5 \text { sites in the } \\
\text { USA + } 7 \text { sites in the USA }\end{array}$ & $1999+2000+2006$ & $\begin{array}{l}5 \text { of } 16 \text { trials } \\
\text { were significant }\end{array}$ & $\begin{array}{l}5 \text { of } 16 \text { trials were } \\
\text { significant }\end{array}$ & $\begin{array}{l}4 \text { of } 16 \text { trials } \\
\text { were significant }\end{array}$ & EFSA-GMO-NL-2008-52 \\
\hline $\mathrm{FG}_{72}$ (untreated) & Jack (II) & $\begin{array}{l}10 \text { sites in North America } \\
6 \text { sites in North America }\end{array}$ & $\begin{array}{l}2008 \\
2009\end{array}$ & $\begin{array}{l}\text { NS } \\
\text { NS }\end{array}$ & $\begin{array}{l}\downarrow_{7 \%} \% \\
\downarrow_{17 \%} \%\end{array}$ & $\begin{array}{l}\uparrow_{13 \%} \% \\
\uparrow_{11} \%\end{array}$ & EFSA-GMO-BE-2011-98 \\
\hline $\mathrm{FG}_{72}$ (isoxaflutole and glyphosate treated) & Jack (II) & $\begin{array}{l}10 \text { sites in North America } \\
6 \text { sites in North America }\end{array}$ & $\begin{array}{l}2008 \\
2009\end{array}$ & $\begin{array}{l}\text { NS } \\
\text { NS }\end{array}$ & $\begin{array}{l}\downarrow_{10 \%} \\
\downarrow_{20 \%}\end{array}$ & $\begin{array}{l}\uparrow_{11 \%} \% \\
\uparrow_{14} \%\end{array}$ & EFSA-GMO-BE-2011-98 \\
\hline A5547-127 X FG72 (untreated) & $\begin{array}{l}\mathrm{MST}_{24} \text { (7 sites) and } \mathrm{MST}_{29} \\
\text { (2 sites) }\end{array}$ & 9 sites in the USA & 2012 & $(\uparrow 13 \%)$ & NS & NS & EFSA-GMO-NL-2013-120 \\
\hline $\begin{array}{l}\text { A5547-127 x FG72 (isoxaflutole+glyphosate+ } \\
\text { glufosinate-treated) }\end{array}$ & $\begin{array}{l}\mathrm{MST}_{24} \text { ( } 7 \text { sites) and } \mathrm{MST}_{29} \\
(2 \text { sites) }\end{array}$ & 9 sites in the USA & 2012 & $\uparrow_{15 \%}$ & NS & NS & EFSA-GMO-NL-2013-120 \\
\hline 305423 (untreated) & Jack (II) & 10 sites in the USA & 2011 & $(\uparrow 38 \%)$ & $\left(\uparrow_{15 \%)}\right.$ & $\uparrow_{36 \%}$ & EFSA-GMO-NL-2007-45 \\
\hline GM event & Control (maturity class) & Location & Year & Total Daidzein & Total Genistein & Total Glycitein & Reference \\
\hline
\end{tabular}




\begin{tabular}{|c|c|c|c|c|c|c|c|}
\hline 305423 (treated with ALS-inhibiting herbicides) & Jack (II) & $\begin{array}{l}9 \text { sites in the USA and } 1 \text { site in } \\
\text { Canada }\end{array}$ & 2011 & $(\uparrow 36 \%)$ & $\left(\uparrow_{11 \%)}\right.$ & $\uparrow 35 \%$ & EFSA-GMO-NL-2007-45 \\
\hline $305423 \times 40-3-2$ (untreated) & Jack (II) & $\begin{array}{l}9 \text { sites in the USA and } 1 \text { site in } \\
\text { Canada }\end{array}$ & 2011 & $(\uparrow 9 \%)$ & NS & $\downarrow 8 \%$ & EFSA-GMO-NL-2007-47 \\
\hline $\begin{array}{l}305423 \times 40-3-2 \text { (chlori-muron + thifensulfuron + } \\
\text { glyphosate)-treated }\end{array}$ & Jack (II) & $\begin{array}{l}9 \text { sites in the USA and } 1 \text { site in } \\
\text { Canada }\end{array}$ & 2011 & $(\uparrow 8 \%)$ & NS & $\downarrow 6 \%$ & EFSA-GMO-NL-2007-47 \\
\hline 356043 (untreated) & Jack (II) & $\begin{array}{l}4 \text { sites in the USA and } 2 \text { in Canada } \\
4 \text { sites in Chile and } 2 \text { in Argentina }\end{array}$ & $\begin{array}{l}2005 \\
2005 / 2006\end{array}$ & $\begin{array}{l}\uparrow_{12 \%} \% \\
\text { NS }\end{array}$ & $\begin{array}{l}\text { NS } \\
\text { NS }\end{array}$ & $\begin{array}{l}\text { NS } \\
\text { NS }\end{array}$ & EFSA-GMO-NL-2007-43 \\
\hline $\begin{array}{l}356043 \text { (treated with glyphosate + ALS-inhibiting } \\
\text { herbicides) US (treated with glyphosate) (treated } \\
\text { with chlorimuron and/or thifensulfuron) (treated } \\
\text { with glyphosate + chlorimuron and/or } \\
\text { thifensulfuron) = ALS-inhibiting herbicides }\end{array}$ & Jack (II) & $\begin{array}{l}4 \text { sites in the USA and } 2 \text { in Canada } \\
4 \text { sites in Chile and } 2 \text { in Argentina }\end{array}$ & $\begin{array}{l}2005 \\
2005 / 2006\end{array}$ & $\begin{array}{l}\text { NS } \\
\uparrow_{13 \%} \\
\text { NS } \\
\uparrow_{15 \%}\end{array}$ & $\begin{array}{l}\text { NS } \\
\text { NS } \\
\text { NS } \\
\text { NS }\end{array}$ & $\begin{array}{l}\uparrow 29 \% \\
\uparrow 21 \% \\
\uparrow 23 \% \\
\text { NS }\end{array}$ & EFSA-GMO-NL-2007-43 \\
\hline DAS-44406-6** & Maverick (III) & 10 sites in the USA & 2010 & NS** & NS** & NS** & Lepping et al., 2013 \\
\hline DAS-81419-2 ${ }^{b}$ & Maverick (III) & 10 sites in the USA & 2011 & NS & NS & $(\downarrow 9 \%)$ & EFSA-GMO-NL-2013-116 \\
\hline DAS-68416-4 & Maverick (III) & 8 sites in the USA & 2009 & NS & NS & $(\uparrow 2-3 \%)^{\mathrm{a}}$ & Herman et al., 2011 \\
\hline DAS-68416-4 × MON-89788-1** (untreated) & Maverick x $\mathrm{A}_{3244}$ & 10 sites in the USA & 2011 & NS & NS & $\left(\uparrow_{11 \%)}\right.$ & EFSA-GMO-NL-2013-115 \\
\hline $\begin{array}{l}\text { DAS- } 68416-4 \times \mathrm{MON}-89788-1^{* *} \text { (treated with } \\
2,4-\mathrm{D} \text {, glufosinate, or glyphosate, or a } \\
\text { combination of these) }\end{array}$ & Maverick x A3244 & 10 sites in the USA & 2011 & NS & NS & $(\uparrow 9-12 \%)$ & EFSA-GMO-NL-2013-115 \\
\hline BPS-CV127-9 (untreated) & Conquista & 7 sites in Brazil + 1 site in the USA & $2009 / 2010+2011$ & $(\downarrow 28 \%)$ & $\left(\downarrow_{17 \%)}\right.$ & NS & EFSA-GMO-NL-2009-64 \\
\hline BPS-CV127-9 (imidazolinone-treated) & Conquista & 7 sites in Brazil + 1 site in the USA & $2009 / 2010+2011$ & $(\downarrow 30 \%)$ & $\left(\downarrow_{19 \%)}\right.$ & NS & EFSA-GMO-NL-2009-64 \\
\hline SYHToH2 (untreated) & Jack (II) & 8 sites in the USA & 2010 & NS & NS & NS & EFSA-GMO-DE-2012-111 \\
\hline SYHToH2 (mesotrione- and glufosinate-treated) & Jack (II) & 8 sites in the USA & 2010 & NS & NS & NS & EFSA-GMO-DE-2012-111 \\
\hline
\end{tabular}

Note: $\quad$ *Not treated with target herbicide (glyphosate).

**Five herbicide treatment regimens. There was no difference between DAS-44406-6 and Maverick for any of the five comparisons.

a NS in untreated and 2,4-D-treated soybean DAS-68416-6 but significant in soybean DAS 68416-6 treated with glufosinate or glufosinate in combination with 2,4-D.

b The pat gene was considered a selectable marker.

$c<$ The maintenance treatment of the field trial included the conventional herbicides quizalofop p-ethyl and fomesafen for all plots except those given the intended herbicide. 
Understanding the maturation cycle for a crop is important when selecting appropriate varieties to cultivate in a specific region. As described by one of the plant breeding companies (https://www.pioneer.com/home), soybeans flower in response to day length. When days begin to shorten in the northern hemisphere after midsummer, day-length sensitive soybean varieties are triggered into flowering. Soybeans are therefore referred to as short-day plants. Summer days are longer in the north, and northern varieties are adapted to these longer days. Therefore, if a northern variety is moved south, it will flower earlier than it would in its area of adaptation. Accordingly, they will usually yield less than adapted varieties that use the entire growing season. If moved too far south, it may flower extremely early before reaching adequate height. A southern variety moved north will flower later in the season when the day length shortens to that of its southern area of adaptation and hence be at risk of a killing frost. Soybeans have been bred for adaptation to relatively narrow maturity ranges. Varieties in each maturity group will usually perform as fullseason varieties within a band that is no wider than 150 to $250 \mathrm{~km}$ from north to south. There are in total 13 maturity groups for soybean, from the most northern varieties in group ooo to most southern varieties in group $X$. Because there is no standard on how to establish the maturity class of each new soybean variety, each plant breeder has established benchmark products to help evaluate the relative maturity of its entire product line. From what is said above, it is obvious that a comparative risk assessment of a GM plant needs to be done with a non-GM plant variety of a comparable genetic background, including equivalent maturing characteristics. The comparator and maturity class for each specific GM soybean is given in the second column of Table 13 (the maturity class appears within brackets).

Because most compositional information on GM soybeans is available for the Monsanto event 40-3-2, some historical information on this event is given before the isoflavone data in GM soybeans are discussed more generally. Monsanto has been involved in the development of glyphosate-tolerant crops since the early 1980 s (Padgette et al., 1996). The research and development unit of the company developed a strategy to obtain tolerance to glyphosate known as the target-site approach in which an herbicide-insensitive target protein is identified. The gene coding for it is isolated and introduced into crop plants through genetic engineering. Glyphosate specifically binds to and blocks the activity of the endogenous enzyme 5enolpyruvylshikimate-3-phosphate synthase (EPSPS), an enzyme of the aromatic amino-acid biosynthetic pathway available in the chloroplast or plastid (Della-Cioppa et al., 1986). EPSPS enzymes are available in all plants, bacteria, and fungi but not in animals. Animals cannot make their own aromatic amino-acids but obtain them from their diet; these amino acids are therefore called essential. The binding of glyphosate to EPSPS inhibits the enzyme and prevents the plant from synthesising the aromatic amino acids phenylalanine, tyrosine, and tryptophan and leads to death. Plants have been transformed with a gene coding for a glyphosate-insensitive enzyme isolated from the soil bacterium Agrobacterium sp. strain $\mathrm{CP}_{4}$ (CP4 EPSPS) fused to a chloroplast transit peptide. The first such plant introduced on the market was a glyphosate-tolerant soybean called 40-3-2. Although glyphosate inhibits the activity 
of the endogenous EPSPS enzyme, the insensitive $\mathrm{CP}_{4}$ EPSPS enzyme expressed allows the transformed soybean to produce aromatic amino acids and survive after treatment with glyphosate-containing herbicides (Delannay et al., 1995; Padgette et al., 1995, 1996). Because surrounding weeds do not have glyphosate insensitive EPSPS enzymes, they are killed by glyphosate.

The lack of alteration of aromatic amino acid levels in soybean 40-3-2 as compared with soybean $\mathrm{A}_{5403}$, a conventional non-GM soybean with comparable genetic background, is in accordance with the expectation that 5enolpyruvylshikimate-3-phosphate synthase is not the rate-limiting step in aromatic amino acid biosynthesis in bacteria and plants (Herrmann, 1983).

In the first compositional studies of soybean 40-3-2 (not sprayed with glyphosate), the investigators not only studied the composition of the raw soybean seeds but also the composition of processed products such as toasted soybean meal (Padgette et al., 1996). Although the investigators noted an extremely large range of values for the isoflavones genistein and daidzein, the toasted meal contained comparable levels of isoflavones and other phytoestrogens (total genistein, total daizdein, total coumestrol, and biochanin A) to that of toasted meal of the control soybean $\mathrm{A}_{5403}$ and mirrored those levels in the unprocessed soybeans. The investigators concluded that the composition of glyphosate tolerant soybean seeds and the processed fractions (toasted meal, defatted meal, protein isolate, protein concentrate, refined bleached and deodorised oil, and lecithin) is comparable to those of the control soybean seeds and fractions (Padgette et al., 1996). Later, Taylor et al. (1999) showed that the conclusions also holds for soybean 40-3-2 sprayed with its target herbicide glyphosate. It had previously been reported that the level of isoflavones in conventional glyphosate-sensitive plants may be increased when treated with this herbicide (Sanderman \& Wellerman, 1988). The study of Taylor et al. (1999) indicated that this increase in isoflavone levels could be the result of a stressinduced response to the application of glyphosate to the non-glyphosate-tolerant soybeans. The application of glyphosate to the glyphosate-tolerant soybean 40-3-2 does not cause such a stress response as demonstrated by the yield (Delannay et al., 1995) and compositional data (Padgette et al., 1996; Taylor et al., 1999).

Monsanto has maintained annual compositional analysis programmes for soybean $40-3-2$ in North America for at least 15 years. These programs have involved measurement of key components such as isoflavones from different 40-3-2 varieties (the event crossed into various commercial genetic backgrounds of different maturity classes) grown across diverse geographical regions. McCann et al. (2005) and Zhou et al. (2011a) published compositional data from 112 unique soybean varieties grown over at least nine growing seasons (2000 to 2009) in geographical areas encompassing the USA, Canada, South America, and Europe. Values for 2000 and 2001 were based on $25 \mathrm{GM}$ and control varieties, values for 2002 on $16 \mathrm{GM}$ and control varieties, values for 2003-2008 on 12 GM and control varieties, and values for 2009 on 11 GM and control varieties. Because it is typical for nutrients and anti-nutrients in crops to vary depending on variety (genetic background), the environmental conditions and cultivation method used (Setchell \& Cole, 2003; De Mejia et al., 2003; Zhou et al., 
2011b), and the glyphosate-tolerant and control soybeans in these studies were not matched for genetic background and were grown under different environmental conditions (not grown at the same field trial site during the same season), the studies were not appropriately designed to compare whether the introduction of the glyphosate tolerant trait in the soybean had resulted in altered levels of isoflavones. Nevertheless, the study indicates that the isoflavone composition of commercialised glyphosate-tolerant soybeans over several years of breeding into multiple genetic backgrounds remains substantially equivalent to that of conventional soybeans. The levels of the various isoflavones in conventional soybeans have already been summarised in subsection Conventional soybeans in section 3.1.7 Isoflavones in legumes. The average mean daidzein content for 40-3-2 and control soybeans grown from 2000 to 2009 was 736.71 and $740.60 \mu \mathrm{g} / \mathrm{g} \mathrm{dw}$, respectively. For genistein it was 830.74 and $830.27 \mu \mathrm{g} / \mathrm{g} \mathrm{dw}$, respectively, and for glycitein 152.86 and $147.57 \mu \mathrm{g} / \mathrm{g} \mathrm{dw}$, respectively. There were also similar levels of these isoflavones in 40-3-2 and the conventional soybeans each year of the study.

The rapid adoption of Roundup Ready soybean (event 40-3-2) by growers around the world is attributed to the simplicity of use, cost efficiencies for weed control, and compatibility with conservation tillage. Because Roundup agricultural herbicides are highly effective against most annual and perennial grasses and broadleaf weeds, growers planting Roundup Ready soybeans can reduce the number of herbicides used to control weeds that grow in their field and thereby realise savings in weed control costs (Carpenter, 2001). A second-generation glyphosate-tolerant soybean product, MON 89788, expressing the same CP4 EPSPS protein expressed in soybean 40-3-2, was produced in the Asgrow A3244 genetic background. Soybean MON 89788 in the A3244 genetic background has for over four years been shown to have yield advantage over soybean 40-3-2 in the same elite genetic background (Lundry et al., 2008). Soybean MON 89788 is therefore expected to take most, or at least some of the extensive market shares now held by soybean 40-3-2.

Lundry et al. (2008) conducted compositional analyses on seed and forage of soybean MON 89788 and its comparator collected at five field locations in the USA and Argentina over two growing seasons (2005 in the USA, 2004/2005 in Argentina). All 46 constituents analysed in seeds from the Argentinean field trials and 45 of the 49 constituents analysed in seeds from the field trial in the USA showed no statistically significant difference between soybean MON 89788 and its control material (A3244). The four constituents for which the level differed (forage moisture, daidzein, glycetein, and vitamin E) in the field trials in the USA were evaluated in relation to the known variability of these constituents in commercial soybean varieties. It was noted that the magnitude of the differences for daidzein and glycitein was very small (7.5$10.6 \%$ relative to the control), that the level of genistein did not differ, and that all values were within the range normally observed in conventional soybeans (subsection Conventional soybeans in section 3.1.7 Isoflavones in legumes). An extensive variation in isoflavone levels was demonstrated in a study performed in two regions of Brazil over two growing seasons (Zhou et al., 2011b). This study evaluated the relative contribution of growing season, growing region, phenotype (glyphosate tolerance due 
to the MON 89788 event vs. sensitivity), and genotype (genetic background of cultivar) on variability. The range in the level of daidzein (across years, genotypes) in the MON 89788 soybean was $181-1,350 \mathrm{mg} / \mathrm{kg} \mathrm{dw}$ and in the conventional soybeans $172-2,100 \mathrm{mg} / \mathrm{kg} \mathrm{dw}$. The range in level of genistein in the MON 89788 was $132-1,324$ $\mathrm{mg} / \mathrm{kg} \mathrm{dw}$ and in the control $250-1,649 \mathrm{mg} / \mathrm{kg} \mathrm{dw}$. The level of glycitein in the GM was $33-338 \mathrm{mg} / \mathrm{kg} \mathrm{dw}$ and in the control $51-275 \mathrm{mg} / \mathrm{kg} \mathrm{dw}$. The study highlighted extensive differences in isoflavone levels of soybean seeds harvested from the different regions. Levels of isoflavones were higher in the southern soybean growing region of Brazil $(267-2,098 \mu \mathrm{g} / \mathrm{g} \mathrm{dw})$ than in the northern soybean growing region $(172-447 \mu \mathrm{g} / \mathrm{g} \mathrm{dw})$, which is likely due to soybeans of different maturity classes being grown at these regions. The statistical analysis of the data confirmed a strong interaction between several of the parameters studied. Against the background of several hundred percent differences in isoflavone levels in the study of Zhou et al. (2011b), the very small 10\% difference in the study of Lundry et al. (2008) seems trivial. It was concluded that the isoflavone level in soybean MON 89788 is comparable with that of the conventional counterpart and commercial soybean varieties.

A very similar picture with respect to isoflavone content to that occurring in glyphosate-tolerant soybeans (events $40-3-2$ and MON 89788) emerges when the other types of genetically modified soybean mentioned in Table 12 are scrutinised. With a single exception, the content of total daidzein, total genistein, and total glycitein in the GM soybean events was found to be comparable with that in the corresponding comparator having a similar genetic background (except for the newly introduced traits) to the GM event. Because the statistical analysis of compositional studies on 60-100 plant constituents is expected to identify statistically significant differences for a low proportion of the analysed parameters simply by chance, the EFSA GMO Panel has developed a methodology to put any differences identified into a biological context. By including a set of commercial non-GM reference soybean varieties in the field trials with the GM soybean and its comparator, it is possible to establish whether differences occur between the GM plant and its comparator and to conclude on whether levels in the GM plant are equivalent to the levels in the commercial non-GM soybean varieties included in the study (EFSA, 2010, 2011). In Table 13 , the statistically significant increases or decreases in total daidzein, total genistein, or total glycitein identified in the difference testing are placed within brackets when the equivalence test identified the level in the GM plant and its comparator to be within the equivalence limits identified by the commercial non-GM reference soybeans included in the study. These statistically significant differences are in most cases not considered to be of biological relevance. This statistical methodology has been applied in all applications developed after 2011 as well as in some of the earlier ones. It is obvious from Table 13 that nearly all identified differences between a GMO and its comparator have been of small magnitude and occurred at isoflavone levels that were equivalent to those occurring in commercial non-GM soybeans on the market. The only exception identified until now is soybean MON 87769, which consistently showed 30-35\% less total daidzein and total genistein than in its comparator. Nonetheless, the reduced isoflavone levels were still within 
this $99 \%$ tolerance interval of the reference soybean varieties included in the studies. Thus, the extended risk assessment concluded that the reduced isoflavone content of the newly developed soybean constitutes no risk for humans, animals, and the environment.

Statisticians from the biotechnology industry have used alternative statistical methodology (Bayesian statistics) to assess potential differences in composition between transgenic soybeans and their conventional counterparts. Harrison et al. (2013) focused on studying isoflavones and anti-nutrients. These investigators used compositional data from soybean seeds harvested from eight replicated field sites in the major soybean growing region of the USA in 2009. The studied materials were a new GM soybean variety, its conventional counterpart $A 3525$, and sixteen conventional reference varieties. The Bayesian statistical analysis indicated that the estimated mean percentage difference in daidzein between the GM soybean and its near-isogenic control was $3.4 \%$, with a $95 \%$ posterior credible interval of $-0.4 \%$ to $7.2 \%$. It was more than $95 \%$ probable that the difference between means is less than $10 \%$. Similar results and conclusions were drawn for genistein and glycitein. Using the same statistical methodology and compositional data on soybean 40-3-2 harvested in 2000 to 2009, Harrigan and Harrison (2012) concluded that the means and ranges of values for genistein (one of a few compounds studied as markers) in the transgenic soybean were consistently similar to those in the conventional comparator (differences across data were small).

Recently, a few investigators have explored the possibility to use untargeted metabolomics to assess whether the genetic modification has resulted in a distortion of the plants metabolome additional to the pathway(s) intended to be influenced. To account for environmental effects and introgression of GM traits into diverse genetic backgrounds, it has been suggested that the assessment for GM crop metabolic composition be understood within the context of the natural variation for the crop (Clarke et al., 2013b). These investigators profiled 169 metabolites and established their dynamic ranges from the seeds of 49 conventional soybean lines representing the current commercial genetic diversity. It was then possible to demonstrate that the metabolome of a genetically modified soybean had no significant deviation from natural variation within the soybean metabolome, except for changes in the targeted engineered pathway.

In a similar study, Lin et al. (2014) identified 169 metabolites in 29 common soybean varieties. Of the studied metabolites, 104 were found to be significantly variable in their levels across tested cultivars. Some metabolite-metabolite correlations were identified, which could be useful when assessing potential differences between different varieties.

Taking all data available on the isoflavone content in GM soybeans and their nonmodified comparators into consideration, it can be concluded that it is unlikely that the genetic modifications studied so far (with exception of soybean MON 87769) influence the isoflavone concentration in the GM plant. Thus, the isoflavone concentration will be comparable with that found in commercial non-GM soybean varieties. 


\section{Soybean sprouts}

Soybean sprouts and sprouts from other legumes (e.g., alfalfa and chickpeas) are important foods in many East Asian countries and the habit of consuming bean sprouts is becoming popular elsewhere as well. Several investigators have studied what happens with the isoflavones in the germinating seed and the developing sprout. Total isoflavone content (sum of daidzein, genistein, daidzin, and genistin) generally increased in soybean seeds during the initial germination and decreased thereafter. Kim et al. (2004) reported that the maximum was 20-50\% above background levels (the increase was mainly in aglycones) and levels were back at background levels three days after initiation of germination. Zhu et al. (2005), in contrast, concluded that the maximum was reached when the hypocotyl length of the germinated seed was tiny (0.5-3.0 mm, dependent on soybean variety). The latter authors also reported that the most prominent increase was seen for malonylgenistin and malonyldaidzin (2-3 times). Genistein and daidzein contents were highest just after soaking, whereas the amount of glycitein and its $\beta$-glucoside conjugates remained almost the same during germination. However, the magnitude of the increase in isoflavone content was recently reported to be dependent on temperature during germination (Quinhone Junior \& Ida, 2015). A deviating report claims that isoflavone levels decrease in seeds when soybeans are germinated (Jeon et al., 2012).

A strong increase in the isoflavone concentration was observed in germinated seeds of Chickpea (Cicer arietinum L.). In this legume, the increases in isoflavone content were over 100-fold (Wu et al., 2012). The alteration was mainly due to an increase in the level of formononetin and biochanin $A$.

Several investigators have compared the isoflavone levels in the seed with the levels occurring in the developing sprouts. Kim et al. (2003) analysed 66 soybean varieties of sprout beans and the bean sprouts produced from thirty of these varieties for total isoflavone content (daidzein + genistein). The mean level of total isoflavones in bean seeds was $1,209 \mathrm{mg} / \mathrm{kg} \mathrm{dw}$ (range: $247-2,256 \mathrm{mg} / \mathrm{kg} \mathrm{dw}$ ), whereas the sprouts on average contained 1,898 mg isoflavones/kg dw (range: $768-3,343 \mathrm{mg} / \mathrm{kg} \mathrm{dw}$ ), with fairly equal amounts of total daidzein and genistein. Total isoflavone contents of bean sprouts increased gradually during the sprouting period, reaching a maximum level on the 5th day of cultivation, and were higher than in roots (had a ratio that strongly favoured daidzein), cotyledon, and hypocotyl. Lee et al. (2007) made similar observations. A difference in total isoflavone level has also been reported for soybean sprouts grown under light and under darkness (Graham \& Graham, 1996; Kim et al., 2006; Phommalth et al., 2008), but this could not be confirmed by others (Lee et al., 2007).

Lee et al. (2013) determined the levels of six isoflavones and coumestrol in soybean (Glycine max) sprouts at various times after infection by anthracnose (Colletotrichum gloeosporioides). The quantities of daidzin, genistin and malonylgenistin increased to peak concentrations about one week after inoculation, and then decreased. Glycitein, which was not detected in the non-inoculated soybean seed, became detectable at about day six after inoculation. The concentration of daidzein, genistein, and coumestrol increased very slightly, if at all, until day nine after inoculation. By contrast, Jeon et al. (2012) observed that exposure of soybean seeds to 
Aspergillus oryzae significantly decreased the total isoflavone content, but induced coumestrol and glyceollin production. It is not known whether these deviating observations are due to the different organisms stressing the soybean.

\section{Other legumes than soybeans}

Although soybean is the most important legume in the human diet, other legumes, such as peas, the common bean, and peanuts, are very important. The main products of the common bean and peas are seeds, which are harvested either before or after physiological maturity as green pods such as snap beans and haricot verts (also known as "green beans") or as dry beans. Both forms have given rise to an important canning industry (recently, freeze dried food products have appeared on world markets). Most dry bean varieties are consumed after water cooking. There are many different types of the common bean, known, for example, as pinto beans, navy beans, black beans, white beans, or kidney beans. Dried stems and pods have been used as hay for animal feeding (Westphal, 1974). Peanuts, also known as groundnut (Arachis hypogaea), is a crop of global importance. Peanuts are similar in taste and nutritional profile to tree nuts (e.g., hazelnuts and almonds) and are often served in similar ways in Western cuisines. The botanical definition of a "nut" is a fruit whose ovary wall becomes very hard at maturity. Using this criterion, the peanut is not a nut but rather a legume. This is the reason why data on peanuts are presented in this section. For culinary purposes, and in common English language usage, peanuts are usually referred to as nuts.

Besides soybean meal and forage, alfalfa and clover are important rations in animal feed.

As shown in Tables 8 and 10, legumes with a relatively low content of isoflavones include the adzuki bean (Vigna angularis), black-eyed bean (Vigna unguiculata subs. unguiculata), black gram (Vigna mungo), broad bean (Vicia faba), common bean (Phaseoulus vulgaris), cowpea (Vigna unguiculata), lentils (Lens culinaris), lima beans (Phaseolus lunatus), liquorice (Glycyrrhiza glabra), mung bean (Vigna radiate), peas (Pisum sativum), peanuts (Arachis hypogaea), pigeon pea (Cajanus cajan), and tepary bean (Phaseolus acutifolius). Although low in isoflavones, liquorice was relatively rich in formononetin and lima beans in biochanin A. Slightly higher to intermediate levels were found in chick peas (Cicer arietinum), kudzu (Pueraria lobata), lupins (Lupinus mutabilis and L. albus), and runner beans (Phaseolus coccineus). High isoflavone contents were found in soybean (Glycine max) and in red clover (Trifolium trapense) and white clover (Trifolium repens).

Konar et al. (2012), in comparing the level of conjugated and free isoflavones in six legumes (chickpea, red kidney bean, haricot bean, yellow lentil, red lentil, and green lentil), found chickpeas to be the best source of isoflavones among these six legumes, the total content being $3.078 \mathrm{mg} / \mathrm{kg}$. It contained significant amounts of biochanin A and its conjugated form sissotrin. Kidney beans ( $a$ form of the common bean) had the second highest concentration of isoflavones $(1.08 \mathrm{mg} / \mathrm{kg})$ and were particularly rich in genistin $(0.95 \mathrm{mg} / \mathrm{kg})$. The total isoflavone content in the four other legumes was below $0.2 \mathrm{mg} / \mathrm{kg}$. 
There are data on some other legume sprouts than soybean sprouts. Intermediate levels of isoflavones are found in alfalfa and clover sprouts, which are comparatively rich in formononetin (alfalfa sprouts: $3.4-51.7 \mathrm{mg} / \mathrm{kg} \mathrm{fw}$, clover sprouts $22.8-40.2 \mathrm{mg} / \mathrm{kg} \mathrm{fw}$ ).

Leuner and co-workers (2012) undertook a study to analyse isoflavones and coumestrol in 41 neglected tropical and subtropical legumes used as human food or animal feed. The compounds studied were isoflavones biochanin A, daidzein, daidzin, formononetin, genistein, genistin, sissotrin, and ononin, in addition to the coumestan coumestrol. At least one of the isoflavones (or coumestrol) of interest was identified in 27 of the 41 legumes. Genistein and biochanin A were the most abundant compounds. The highest contents of genistein were found in the green parts of Andira macrothyrsa (598 mg/kg dw), the seeds of Pachyrhizus tuberosus ( $250 \mathrm{mg} / \mathrm{kg} \mathrm{dw}$ ), and the green parts of Calopogonium mucunoides ( $184 \mathrm{mg} / \mathrm{kg} \mathrm{dw}$ ). The highest contents of biochanin A were found in the green parts of Cratylia argentea $(76 \mathrm{mg} / \mathrm{kg} \mathrm{dw})$, Calopogonium mucunoides ( $53 \mathrm{mg} / \mathrm{kg} \mathrm{dw}$ ), and the flowers of Andira macrothyrsa ( $40 \mathrm{mg} / \mathrm{kg} \mathrm{dw}$ ). Soybeans were used as reference material and contained a sum of $4,466 \mathrm{mg} / \mathrm{kg}$ total isoflavones. None of the studied species contained as much total isoflavones. However, several species may serve as a promising source of individual compounds. The richest source of daidzin was the green parts of Calopogonium mucunoides, containing $1,457 \mathrm{mg} / \mathrm{kg}$. This plant was also the tropical legume with the highest total isoflavone content $(1,740 \mathrm{mg} / \mathrm{kg})$, but that was substantially lower than in soybean. Ononin was detected in two plants, namely Zornia diphylla $(65.9 \mathrm{mg} / \mathrm{kg} \mathrm{dw})$ and Cratylia argentea $(11.6 \mathrm{mg} / \mathrm{kg} \mathrm{dw})$. Sissotrin was found in four legume species: Calopogonium mucunoides (24.4 mg/kg dw), Leucaena leucocephala (24.4 mg/kg dw), Cratylia argentea (15.0 mg/kg dw), and Macroptilium lathyroides (15.0 mg/kg dw).

\subsubsection{Isoflavones in vegetables and root vegetables}

Like in fruits and berries, isoflavone levels are comparatively low in vegetables and root vegetables. Table 14 shows the levels of genistein, daidzein, glycitein, biochanin $A$, and formononetin in vegetables and root vegetables when the data were given as $\mathrm{mg} / \mathrm{kg} \mathrm{fw}$ by the investigators. Data presented as $\mathrm{mg} / \mathrm{kg} \mathrm{dw}$ is summarised in Table 15 .

Not a single vegetable or root vegetable contains above $1 \mathrm{mg}$ total isoflavones per $\mathrm{kg} \mathrm{fw}$. On a dw basis, spring onion contains $1.63 \mathrm{mg}$ and leek $0.21 \mathrm{mg}$ biochanin $\mathrm{A} / \mathrm{kg}$. Screening various other foods than those presented in the Tables above for the presence of daidzein and genistein, after an initial step converting the diphenolic glycosides into their respective aglycones, Mazur (1998) found nothing in avocado

(Persea americana), tomato (Lycopersicum esculentum), chives (Allium schoenoprasum), celery (Apium graveolens), cucumber (Magnolia acuminatus), eggplant (Solanum melongena), and radish (Raphanus sativus radicula). 
Table 14: Isoflavone content $(\mathrm{mg} / \mathrm{kg} \mathrm{fw})$ in vegetables

\begin{tabular}{|c|c|c|c|c|c|}
\hline Types of food and processing & Genistein & Daidzein & Glycitein & Biochanin A & Formononetin \\
\hline Asparagus (1) & n.q.-0.006 & $0.003-0.578$ & & n.d. & n.d. \\
\hline Asparagus, cooked (2) & n.d. & n.d. & & & \\
\hline Aubergine (2) & 0.007 & 0.001 & & & \\
\hline Aubergine, cooked (2) & 0.006 & 0.003 & & & \\
\hline Avocado (2) & n.d. & n.d. & & & \\
\hline Beetroot (2) & n.d.-n.q. & n.d.-n.q. & n.q. & n.d. & n.q. \\
\hline Broccoli sprout $(1,2)$ & n.d. & n.d. -0.437 & 0.9 & n.d. & n.d. \\
\hline Broccoli sprout, cooked (2) & n.d. -0.002 & n.d.- -0.001 & n.q. & & 0.001 \\
\hline Cabbage, Green $(2,5)$ & n.q. -0.006 & n.q. -0.002 & 0.001 & & 0.001 \\
\hline Cabbage, Green, cooked (2) & n.q. & n.q. & & & \\
\hline Cabbage, Red (2) & n.q. & n.d. & & & \\
\hline Cabbage, Red, cooked (2) & 0.024 & n.d. & & & \\
\hline Cabbage, Savoy (2) & 0.029 & n.d. & & & \\
\hline Cabbage, Savoy, cooked (2) & n.d. & n.d. & & & \\
\hline Cabbage, White (2) & 0.003 & n.d. & & & \\
\hline Cabbage, White, cooked (2) & 0.006 & n.d. & & & \\
\hline Carrot $(1,2,5)$ & n.d. -0.001 & n.d.-n.q. & 0.001 & n.d. & n.q. -0.001 \\
\hline Carrot, cooked $(2,5)$ & n.d.-n.q. & n.d.-n.q. & n.d. & & 0.001 \\
\hline Cauliflower, brussel sprouts (1) & n.d. & n.q. & & n.d. & n.d. \\
\hline Cauliflower, cooked (2) & n.d. & n.d. & & & \\
\hline Celeriac (2) & 0.045 & n.d. & & & \\
\hline Celeriac, cooked (2) & 0.034 & n.d. & & & \\
\hline Celery (2) & n.d. & n.d. & & & \\
\hline Celery, cooked (2) & n.d. & n.d. & & & \\
\hline Chicory (2) & n.d. & 0.001 & & & \\
\hline Collards (6) & 0.015 & 0.004 & n.q. & & n.q. \\
\hline Cucumber (2) & 0.003 & n.d. & & & \\
\hline Fennel (2) & n.d. & n.d. & & & \\
\hline Fennel, cooked (2) & n.d. & n.d. & & & \\
\hline Garlic $(1,5)$ & n.q. -0.143 & n.d.-0.05 & 0.002 & n.q. & n.d.- -0.008 \\
\hline Leek (2) & n.d. & n.d. & & & \\
\hline Leek, cooked (2) & n.d. & n.d. & & & \\
\hline Lettuce (5) & 0.005 & 0.001 & 0.001 & & 0.001 \\
\hline Lettuce, Iceberg (2) & n.d. & n.d. & & & \\
\hline Lettuce, Round (2) & n.d. & n.d. & & & \\
\hline Okra (2) & 0.021 & 0.048 & & & \\
\hline Onion $(2,5)$ & n.d. & n.d.-n.q. & 0.001 & & n.q. \\
\hline Onion, cooked (2) & n.d. & n.d. & & & \\
\hline Parsley (4) & 0.3 & n.d. & n.d. & n.d. & n.d. \\
\hline Parsnip, cooked (2) & $0.01 \pm 0$ & n.d. & n.d. & n.d. & n.d. \\
\hline Pepper, Green (2) & n.d. & n.d. & & & \\
\hline Radish (2) & 0.045 & n.d. & & & \\
\hline Salad cress (2) & n.d. & n.d. & & & \\
\hline Spinach $(2,5)$ & n.d.-0.002 & n.d. -0.003 & 0.001 & & 0.001 \\
\hline Spinach, cooked $(2,5)$ & n.d. -0.001 & n.d.-n.q. & 0.002 & & 0.001 \\
\hline Sweet potatoes $(1,2,5)$ & n.d.-0.001 & n.d.-0.001 & n.d. & & 0.001 \\
\hline Sweet potatoes, cooked (2) & n.d. & n.d. & & & \\
\hline Tapioca (3) & n.d. & n.d. & n.d. & 0.09 & n.d. \\
\hline Tapioca starch (2) & 0.253 & 0.132 & 0.001 & & 0.004 \\
\hline Turnip (2) & n.d. & n.d. & n.d. & n.d. & n.d. \\
\hline Winter squash (5) & 0.02 & 0.01 & 0.0 & & 0.01 \\
\hline Zucchini (5) & n.d. & n.d. & n.d. & & n.d. \\
\hline
\end{tabular}

Note: $\quad$ n.d. = non-detectable; n.q. = non-quantifiable.

Source: (1) Horn-Ross et al., 2000; (2) Liggins et al., 2000b; (3) Kuhnle et al., 2009a; (4) Clarke et al., 2013a; (5) Thompson et al., 2006. 
Table 15: Isoflavone content $(\mathrm{mg} / \mathrm{kg} \mathrm{dw})$ in vegetables

Types of food and processing Genistein Daidzein

Glycitein Biochanin A Formononetin

\begin{tabular}{|c|c|c|c|c|c|}
\hline Asparagus (1) & n.q. -0.006 & $0.003-0.578$ & & n.d. & n.d. \\
\hline Asparagus, cooked (2) & n.d. & n.d. & & & \\
\hline Aubergine (2) & 0.007 & 0.001 & & & \\
\hline Aubergine, cooked (2) & 0.006 & 0.003 & & & \\
\hline Avocado (2) & n.d. & n.d. & & & \\
\hline Beetroot (2) & n.d.-n.q. & n.d.-n.q. & n.q. & n.d. & n.q. \\
\hline Broccoli sprout $(1,2)$ & n.d. & n.d. -0.437 & 0.9 & n.d. & n.d. \\
\hline Broccoli sprout, cooked (2) & n.d. -0.002 & n.d. -0.001 & n.q. & & 0.001 \\
\hline Cabbage, Green $(2,5)$ & n.q. -0.006 & n.q. -0.002 & 0.001 & & 0.001 \\
\hline Cabbage, Green, cooked (2) & n.q. & n.q. & & & \\
\hline Cabbage, Red (2) & n.q. & n.d. & & & \\
\hline Cabbage, Red, cooked (2) & 0.024 & n.d. & & & \\
\hline Cabbage, Savoy (2) & 0.029 & n.d. & & & \\
\hline Cabbage, Savoy, cooked (2) & n.d. & n.d. & & & \\
\hline Cabbage, White (2) & 0.003 & n.d. & & & \\
\hline Cabbage, White, cooked (2) & 0.006 & n.d. & & & \\
\hline Carrot $(1,2,5)$ & n.d. -0.001 & n.d.-n.q. & 0.001 & n.d. & n.q. -0.001 \\
\hline Carrot, cooked $(2,5)$ & n.d.-n.q. & n.d.-n.q. & n.d. & & 0.001 \\
\hline Cauliflower, brussel sprouts (1) & n.d. & n.q. & & n.d. & n.d. \\
\hline Cauliflower, cooked (2) & n.d. & n.d. & & & \\
\hline Celeriac (2) & 0.045 & n.d. & & & \\
\hline Celeriac, cooked (2) & 0.034 & n.d. & & & \\
\hline Celery (2) & n.d. & n.d. & & & \\
\hline Celery, cooked (2) & n.d. & n.d. & & & \\
\hline Chicory (2) & n.d. & 0.001 & & & \\
\hline Collards (6) & 0.015 & 0.004 & n.q. & & n.q. \\
\hline Cucumber (2) & 0.003 & n.d. & & & \\
\hline Fennel (2) & n.d. & n.d. & & & \\
\hline Fennel, cooked (2) & n.d. & n.d. & & & \\
\hline Garlic $(1,5)$ & n.q. -0.143 & n.d.- -0.05 & 0.002 & n.q. & n.d.-0.008 \\
\hline Leek (2) & n.d. & n.d. & & & \\
\hline Leek, cooked (2) & n.d. & n.d. & & & \\
\hline Lettuce (5) & 0.005 & 0.001 & 0.001 & & 0.001 \\
\hline Lettuce, Iceberg (2) & n.d. & n.d. & & & \\
\hline Lettuce, Round (2) & n.d. & n.d. & & & \\
\hline Okra (2) & 0.021 & 0.048 & & & \\
\hline Onion $(2,5)$ & n.d. & n.d.-n.q. & 0.001 & & n.q. \\
\hline Onion, cooked (2) & n.d. & n.d. & & & \\
\hline Parsley (4) & 0.3 & n.d. & n.d. & n.d. & n.d. \\
\hline Parsnip, cooked (2) & 0.01 & n.d. & n.d. & n.d. & n.d. \\
\hline Pepper, Green (2) & n.d. & n.d. & & & \\
\hline Radish (2) & 0.045 & n.d. & & & \\
\hline Salad cress (2) & n.d. & n.d. & & & \\
\hline Spinach $(2,5)$ & n.d. -0.002 & n.d. -0.003 & 0.001 & & 0.001 \\
\hline Spinach, cooked $(2,5)$ & n.d. -0.001 & n.d.-n.q. & 0.002 & & 0.001 \\
\hline Sweet potatoes $(1,2,5)$ & n.d.--0.001 & n.d.-0.001 & n.d. & & 0.001 \\
\hline Sweet potatoes, cooked (2) & n.d. & n.d. & & & \\
\hline Tapioca (3) & n.d. & n.d. & n.d. & 0.09 & n.d. \\
\hline Tapioca starch (2) & 0.253 & 0.132 & 0.001 & & 0.004 \\
\hline Turnip (2) & n.d. & n.d. & n.d. & n.d. & n.d. \\
\hline Winter squash (5) & 0.02 & 0.01 & 0.0 & & 0.01 \\
\hline Zucchini (5) & n.d. & n.d. & n.d. & & n.d. \\
\hline
\end{tabular}

Note: $\quad$ n.d. = non-detectable; n.q. = non-quantifiable.

Source: (1) Kuhnle et al., 2009b; (2) Liggins et al., 200ob; (3) Kuhnle et al., 2007; Mazur \& Adlercreutz, 1998; (5) Franke et al., 1994. 


\subsubsection{Isoflavones in nuts}

Close to a dozen nut species have been analysed for their isoflavone content. The data obtained are summarised in Table 16. All investigations on nuts have used analytical methods intended to measure the total content of the various isoflavone aglycones, genistein, daidzein, glycitein, biochanin A, and formononetin. All data summarised in Table 16 have reported the isoflavone concentration on a $\mathrm{fw}$ basis. The US Department of Agriculture (2008) have compiled the occurrence of bioactive constituents in tree nuts, specifically almonds, Brazil nut, cashew nut, hazelnut, macadamia nut, pecan nut, pine nut, pistachio nut, and walnut (Bolling et al., 2011). Except for pistachio nuts and walnuts the total isoflavone levels (daidzein + genistein + glycitein) were low in tree nuts (n.d.-0.3 mg/kg dw). However, because their data are only based on one or two samples per nut, they need further confirmation. As shown in Table 16, Thompson et al. (2006) only found $1.77 \mathrm{mg}$ total isoflavones (daidzein + genistein + glycitein + biochanin A + formononetin) in pistachio nuts.

In addition to the data in Table 16, Mazur and Adlercreutz (1998) used an analytical method that only allowed the reporting of total genistein, daidzein, glycitein, biochanin A, and formononetin on a dw basis. They found none of these compounds in cashew nuts, hazelnuts, and pistachio nuts.

\subsubsection{Isoflavones in oilseed crops}

As indicated by splitting up Table 17 in two sections, some of the investigators that have analysed isoflavones in oilseed crops have reported the data on a fw basis and others on a dw basis. Oil seeds are usually not very rich in isoflavones. The exception is sunflower seeds that contain approximately $11 \mathrm{mg}$ total genistein, $4.6 \mathrm{mg}$ total daidzein, $0.8 \mathrm{mg}$ glycitein, and small amounts of formononetin per $\mathrm{kg} \mathrm{fw}$. All other oil seed crops contain less than $3 \mathrm{mg}$ total isoflavones.

\subsubsection{Isoflavones in cereals}

Such cereals as barley, maize, millet, oat, rye, wheat, and triticale are all very low in the content of isoflavones. Available data on these products when presented as $\mathrm{mg} / \mathrm{kg}$ $\mathrm{fw}$ are shown in Table 18. Table 19 highest isoflavone levels were found in walnuts. shows data presented by the investigators in $\mathrm{mg} / \mathrm{kg} \mathrm{dw}$. Processed products of these cereals might contain some isoflavones but the levels are still low. Data on cereal-based food products such as bread, cookies, and cakes are presented in section 3.1.12, which deals with isoflavone content in other foodstuffs. 
Table 16: Isoflavone content $(\mathrm{mg} / \mathrm{kg} \mathrm{fw})$ in nuts

\begin{tabular}{|c|c|c|c|c|c|}
\hline Type of food. and processing & Genistein & Daidzein & Glycitein & Biochanin A & Formononetin \\
\hline Almonds $(1,2,3,4)$ & n.d.-0.144 & n.d. -0.04 & n.d.-0.006 & 0.25 & n.d.-0.008 \\
\hline Almond, marzipan (5) & n.d. & n.d. & n.d. & n.d. & n.d. \\
\hline Brazil nuts $(2,4)$ & n.d. -0.85 & $0.012-0.06$ & n.d. & 0.13 & n.d. \\
\hline Cashew nuts $(3,4)$ & $0.02-0.103$ & n.d. -0.014 & $0.004-0.01$ & 0.07 & $0.02-0.1$ \\
\hline Chestnuts $(2,3)$ & $0.029-0.164$ & $0.028-0.039$ & 0.003 & & 0.017 \\
\hline Chestnut, cooked (2) & 0.0041 & n.d. & & & \\
\hline Coconut $(2,4)$ & n.d. -0.02 & n.d. -0.076 & n.d. -0.04 & n.d. -0.06 & n.d.-n.d. \\
\hline Hazelnut $(2,3,4)$ & $0.09-0.248$ & n.d. -0.055 & n.d.-0.005 & 0.12 & n.d. -0.012 \\
\hline Pecans $(3,4)$ & $0.009-0.02$ & n.d. -0.016 & n.d.-0.003 & 0.3 & $0.007-0.02$ \\
\hline Pine nuts $(4,5)$ & n.d.-o.04 & n.d. & n.d. & n.d. -0.27 & n.d. \\
\hline Pistachios (3) & 1.033 & 0.731 & 0.004 & & 0.002 \\
\hline Pistachios, roasted and salted (4) & 0.02 & n.d. & 0.02 & 0.27 & 0.02 \\
\hline Walnuts $(1,2,3,4)$ & n.d. -35 & n.d. -27 & n.d. -14 & 3 & n.d. -0.05 \\
\hline
\end{tabular}

Note: $\quad$ n.d. = non-detectable; n.q. = non-quantifiable.

Source: (1) Mazur \& Adlercrutz 1998; (2) Liggins et al., 2000a; (3) Thompson et al., 2006; (4) Kuhnle et al., 2008b; (5) Clarke et al. $2013 a$.

Table 17: Isoflavone content (section A: $\mathrm{mg} / \mathrm{kg} \mathrm{fw}$; section B: $\mathrm{mg} / \mathrm{kg} \mathrm{dw}$ ) in oilseed crops

$\begin{array}{llllll}\text { Types of food and processing } & \text { Genistein } & \text { Daidzein } & \text { Glycitein } & \text { Biochanin A } & \text { Formononetin } \\ \text { Section A }-\mathbf{m g} / \mathbf{k g} \text { fresh weight (fw) } & & & & & \\ \text { Flaxseed (5,6) } & \text { n.d.-1.732 } & \text { n.d.-0.582 } & 0.02-0.56 & 0.06 & 0.01-0.341 \\ \text { Rapeseed (6) } & 0.02 & \text { n.d. } & 0.03 & 0.04 & 0.03 \\ \text { Sesame seed (4,5,8) } & \text { n.d.-0.022 } & \text { n.d.-0.037 } & 0.02 & \text { n.d. } & \text { n.d.-0.038 } \\ \text { Sesame oil (7) } & \text { n.d. } & \text { n.d. } & \text { n.d. } & \text { n.d. } & \text { n.d. } \\ \text { Sunflower seed (2) } & 11.115 & 4.615 & 0.812 & & 0.019 \\ \text { Section B - mg/kg dry weight (dw) } & & & & & \text { n.d. } \\ \text { Flaxseed (1,2) } & \text { n.d. } & \text { n.d. } & & & \\ \text { Flaxseed, crushed (2) } & \text { n.d. } & \text { n.d. } & & & \\ \text { Sesame seed (2) } & 0.14 & 1.4 & & & \\ \text { Sunflower seed (3,4,5) } & 42.9 & 62.7 & & & \end{array}$

Note: n.d. = non-detectable; n.q. = non-quantifiable.

Source: (1) Mazur et al. 1996; (2) Mazur \& Adlercrutz 1998; (3) Horn-Ross et al. 2000; (4) Liggins et al., 2000a; (5) Thompson et al., 2006; (6) Kuhnle et al., 2008b; (7) Clarke et al. 2013a; (8) Franke et al., 1994. 
Table 18: Isoflavone content $(\mathrm{mg} / \mathrm{kg} \mathrm{fw})$ in cereal products

\begin{tabular}{llllll} 
Types of food and processing & Genistein & Daidzein & Glycitein & Biochanin A & Formononetin \\
\hline Pearl barley (5) & n.q & n.d. & n.d. & 0.14 & n.d. \\
Maize (4) & 0.034 & 0.015 & 0.001 & & 0.001 \\
Sweet maize (3) & n.d.-0.02 & n.d.-0.024 & n.d.-0.005 & n.d. & n.d. \\
Sweet maize, cooked (6) & n.d.-0.001 & n.d.-0.001 & n.d. & n.d.-0.01 & n.d.-0.001 \\
Maize flour (5) & 0.06 & n.d. & n.d. & n.d. & n.d. \\
Maize meal (5) & n.q & n.d. & n.d. & 0.08 & n.d. \\
Millet (2) & n.d. & n.d. & n.d. & n.d. & n.d. \\
Oat meal (5) & n.q & n.d. & n.d. & 0.06 & n.d. \\
Oat, bran/germ (5) & n.d. & n.d. & n.d. & 0.07 & n.d. \\
Rice (4,5) & n.d.-0.02 & n.d.-0.007 & n.d.-0.01 & n.d.-0.07 & n.d.-0.02 \\
Wheat grain (1) & o.032 & n.d. & & & \\
Wheat bran (1,5) & n.d.-0.011 & o.007-0.2 & 0.3 & n.d. & n.d. \\
Wheat flakes (6) & n.q. & 0.033 & & & \\
Wheat flour (5) & n.d. & n.d. & & & \\
Wheat meal (1) & o.017-0.018 & o.011-0.023 & & & \\
Wheat germ (2,5) & n.d.-0.02 & n.d. & n.d. & 0.02 & 0.01 \\
\hline
\end{tabular}

Note: $\quad$ n.d. $=$ non-detectable; n.q. $=$ non-quantifiable.

Source: (1) Mazur and Adlercreutz. 1998; (2) Clarke et al.. 2013a; (3) Kuhnle et al.. 2007; (4) Thompson et al.. 2006; (5) Kuhnle et al.. 2009a; (6) Liggins et al.. 2002.

Table 19: Isoflavone content $(\mathrm{mg} / \mathrm{kg} \mathrm{dw})$ in cereal products

\begin{tabular}{llllll} 
Types of food and processing & Genistein & Daidzein & Glycitein & Biochanin A & Formononetin \\
\hline Barley, grain (1) & 0.08 & 0.14 & & & \\
Barley, bran (1) & 0.16 & 0.06 & & & \\
Pearl barley (6) & 0.086 & n.d. & & & \\
Sweet maize (6) & 0.02 & n.d. & n.d. & 0.01 & n.d. \\
Sweet maize, cooked (4) & n.d.-0.14 & n.d.-0.08 & & n.d. & 0.26 \\
Maize meal (6) & 0.142 & 0.095 & & & \\
Maize flour (6) & 0.051 & 0.058 & & & \\
Oat meal (1,6) & n.d. & n.d. & & & \\
Oat meal, boiled (6) & n.d. & n.d. & & & \\
Oat, bran (1) & n.d. & n.d. & & & \\
Rice (6) & n.d. & n.d. & & & \\
Rice, boiled (6) & 0.079 & o.119 & & & \\
Rice, brown. grain (2,6) & $0.713-0.76$ & 0.55-0.613 & 0.07 & 0.05 & n.d. \\
Rice, brown. boiled (2,6) & n.d.-0.141 & n.d.-0.125 & n.d. & n.d. & n.d. \\
Rye meal, grain (1) & n.d. & n.d. & & & \\
Rye bran (1) & n.d. & n.d. & & & \\
Triticale meal (1) & n.d. & n.d. & 0.01 & 0.06 & n.d. \\
Triticale, grain (1) & n.d. & n.d. & 0.01 & 0.07 & n.d. \\
Wheat bran (6) & o.02 & n.d. & n.d. & n.d. & n.d. \\
Wheat flour (5,6) & n.d.-0.02 & n.d.-0.02 & n.d.-0.01 & $0.03-0.09$ & n.d. \\
Wheat germ (3) & n.d.-0.02 & n.d.-0.03 & n.d. & n.d. & n.d. \\
\hline
\end{tabular}

Note: $\quad$ n.d. $=$ non-detectable; n.q. $=$ non-quantifiable.

Source: (1) Mazur \& Adlercreutz. 1998; (2) Kaufman et al.. 1997; (3) Knuckles et al. . 1976; (4) Kuhnle et al. 2007; (5) Kuhnle et al.. 2009a; (6) Liggins et al.. 2002.

\subsubsection{Isoflavones in other foodstuffs}

This category includes various processed products and foodstuffs not easily placed in the food groups discussed above, but which might give a non-neglectable contribution to the exposure of consumers via food (or feed) to one or more 
isoflavones. The data on this group of foodstuffs are shown in Table 20. It should be noted that in many cases it is not possible to identify which ingredient contributes to the isoflavone levels in these foodstuffs. However, in cases in which one of the ingredients in the food is a soybean product, it seems reasonable to assume that this ingredient gives a considerable contribution to the total isoflavone content. This assumption is confirmed by the data in Table 20. A variety of soy foods are consumed in Asia, including tofu, miso, soymilk, soy sauce, soy flour, green or dried soybeans, soybean sprouts, fermented soy food called natto, soy meat patties, soy cheese, soy ice cream, and soy yogurt (Holt, 1997).

Setchell and Cole (2003) measured the total isoflavone content of two types of soy protein isolate over a three-year period, as well as of 85 samples of forty brands of soy milk. Total isoflavone concentrations differed markedly between the soy protein isolates, varying by $200-300 \%$ over three years, whereas the protein content varied by only $3 \%$. Total isoflavone content varied by up to fivefold among different commercial soy milks and was not consistent between repeat purchases. Whole soybean milks had significantly higher isoflavone levels $(63.3 \mathrm{mg} / \mathrm{L})$ than those made from soy protein isolates $(30.2 \mathrm{mg} / \mathrm{L})$, although some isolated soy protein-based milks were similar in content to "whole bean" varieties. The ratio of genistein to daidzein isoflavone forms was higher in isolated soy protein-based milks (2.72) versus "whole bean" soy milks (1.62). The greatest variability in isoflavone content was observed among brands of whole bean soy milks.

Isoflavone content in soymilk prepared from germinated soybean (at least 12 hours) was higher than when prepared directly from soybean seeds (Lee et al., 2005), $5,552 \mathrm{mg} / \mathrm{kg} \mathrm{dw}$ versus $1,612 \mathrm{mg} / \mathrm{kg} \mathrm{dw}$. A soy milk analysed by Song et al. (1998) contained 2,140 $\mathrm{mg}$ total isoflavones per $\mathrm{kg}$.

Song et al. (1998) analysed soybeans and a set of foodstuffs containing soybean products as ingredients for twelve isoflavones, i.e. daidzein, genistein, and glycitein, as well as their glucosides, malonylglucosides, and acetylglucosides. In general, the malonylisoflavones were most common, followed by the glucosylated isoflavones, the aglycones, and the acetylisoflavones. Of the malonylglucosides, those of geinstin and daidzin were much more common than those of glycitin. Seeds of the analysed soybean contained 1,830 mg/kg total isoflavones. Textured soy protein contained 1,435 mg/kg fw total isoflavones and soy germ as much as $23,201 \mathrm{mg} / \mathrm{kg} \mathrm{fw}$. Of traditional soyfoods, tofu contained 1,924 mg/kg fw, tempeh 1,910 $\mathrm{mg} / \mathrm{kg} \mathrm{fw}$, and miso 1,534 mg/kg fw.

Not unexpectedly, soy-based hamburger bread (259 mg/kg fw), mince $(286 \mathrm{mg} / \mathrm{kg}$ $\mathrm{fw})$, miso paste (111 mg/kg fw), soft tofu (225-280 mg/kg fw), tempeh (182 mg/kg fw), ice cream ( $135 \mathrm{mg} / \mathrm{kg} \mathrm{fw})$, and yogurt ( $102 \mathrm{mg} / \mathrm{kg} \mathrm{fw}$ ) were among the foodstuffs with more than $100 \mathrm{mg}$ total isoflavones/kg fw (Franke et al., 1994; Horn-Ross et al., 2000; Liggins et al., 2000b; Thompson et al., 2006; Kuhnle et al., 2008a; Clarke et al., 2013a). Other foodstuffs in this category were black bean sauce ( $53-152 \mathrm{mg} / \mathrm{kg} \mathrm{fw})$, soy paste (57-147 mg/kg dw) and defatted soy meal $(1,369 \mathrm{mg} / \mathrm{kg} \mathrm{dw})$, the latter being used as animal feed (Wang et al., 1990; Thompson et al., 2006; Clarke et al., 2013a). The high level of isoflavones in miso and tofu was confirmed in studies giving the content in $\mathrm{mg} / \mathrm{kg} \mathrm{dw}$ (Wang et al., 1990; Song et al., 1998; Wu et al., 2012). 
Lee et al. (2002) analysed various soybean products for their total isoflavone contents. It was demonstrated that chongkukjang, soybean paste, and miso contained $1,045,781$, and 1,067 mg/kg dw, respectively. In hard, semi-soft, soft and fried tofu the total isoflavone contents were $650,895,685$, and $616 \mathrm{mg} / \mathrm{kg} \mathrm{dw}$, respectively.

Fukutake et al. (1996) measured the genistein and genistin content in soybeans, soy nuts, and soy powder to be in the range $4.6-18.2$ and $200.6-968.1 \mathrm{mg} / \mathrm{kg} \mathrm{dw}$, respectively, for these isoflavones. Soy milk and tofu (bean curd) contained less, 1.913.9 and $94.8-137.7 \mathrm{mg} / \mathrm{kg} \mathrm{dw}$, respectively. Levels in fermented soybeans products such as miso (bean paste) and natto (fermented soybeans) were $38.5-229.1 \mathrm{mg} / \mathrm{kg} \mathrm{dw}$ for genistein and $71.7-492.8 \mathrm{mg} / \mathrm{kg} \mathrm{dw}$ for genistin. It was concluded that the level of genistein in the fermented soybean products was higher than in soybeans and soybean products such as soy milk and tofu. The investigators suggested that the $\beta$ glycosyl bond of genistin is cleaved to produce genistein by microbes during fermentation to yield miso and natto.

Besides foods with legume ingredients, most other foodstuffs in this category contain low levels of isoflavones. For example, flour, bread, and cookies usually contain low amounts of isoflavones, as does cakes and biscuits (Table 20 and 21).

There is limited information on the isoflavone content of mushrooms or mushroom products. The cultivated mushroom, Agaricus bisporus, contains low levels of genistein ( $0.016 \mathrm{mg} / \mathrm{kg} \mathrm{fw}$ ) and daidzein (0.001 mg/kg fw) (Liggins et al., 2000b). No data have been found on other mushrooms. No isoflavones were found in a protein preparation called quorn and obtained from microfungi (Kuhnle et al., 2008a; Clarke et al., 2013a).

Six spices have been analysed for isoflavones. Only canned chilli contained appreciable amounts of these constituents: $7.14 \mathrm{mg}$ total genistein, $5.43 \mathrm{mg}$ total daidzein, and $0.35 \mathrm{mg}$ total biochanin A (Horn-Ross et al., 2000).

Table 20: Isoflavone content $(\mathrm{mg} / \mathrm{kg} \mathrm{fw})$ in other types of food

\begin{tabular}{|c|c|c|c|c|c|}
\hline Types of food and processing & Genistein & Daidzein & Glycitein & Biochanin A & Formononetin \\
\hline Arrow root (10) & 0.02 & n.d. & n.d. & n.d. & n.d. \\
\hline Black rice mochi (1) & n.d. & n.d. & n.d. & n.d. & n.d. \\
\hline Bread $(2,5,8,10,11)$ & n.d. -68.07 & n.d. -30.65 & n.d.-7.09 & n.d. -0.20 & n.d.- -0.08 \\
\hline Burgen bread, soya-based (1) & 149 & 84 & 26 & n.d. & n.d. \\
\hline Cereal $(8,10,11)$ & n.d. -0.760 & n.d. -0.372 & n.d.- -0.22 & & $0.005-0.029$ \\
\hline Cereal, cooked (8) & 0.013 & 0.007 & 0.001 & & 0.002 \\
\hline Chilli, canned (2) & 7.14 & 5.43 & & 0.35 & n.d. \\
\hline Chutney, Victorian (1) & n.d. & n.d. & 0.9 & n.d. & n.d. \\
\hline Chinese black bean sauce (2) & 55.77 & 96.17 & & n.d. & n.d. \\
\hline Cold cereal (2) & n.d. & 0.54 & & n.d. & n.d. \\
\hline Cookies, biscuits $(2,4,8,10)$ & n.d. -0.288 & n.d. -0.29 & n.d. -0.004 & n.d. & n.d. -0.006 \\
\hline Couscous $(1,8)$ & n.d. -0.008 & n.d. -0.05 & n.d. -0.002 & n.d. & n.d. -0.003 \\
\hline Crumpet (1) & n.d. & n.d. & n.d. & n.d. & n.d. \\
\hline Cultivated mushroom (4) & 0.016 & 0.001 & & & \\
\hline Cultivated mushroom, cooked (4) & 0.015 & n.d. & & & \\
\hline Baby formula (3) & 0.11 & 0.03 & 0.03 & n.d. & 0.02 \\
\hline Brown flour (11) & $0.083-0.255$ & $0.082-0.279$ & & & \\
\hline Cake (8) & 0.004 & 0.001 & 0.001 & & 0.002 \\
\hline Chocolate mousse (3) & n.d. & n.d. & n.d. & n.d. & n.d. \\
\hline Chicken noodle soup (8) & 0.001 & n.d. & n.d. & & 0.001 \\
\hline Coffee-mate powder (3) & 0.06 & 0.01 & 0.02 & n.d. & 0.01 \\
\hline Dessert topping, canned (3) & 0.03 & 0.01 & n.d. & n.d. & n.d. \\
\hline Diet shakes/nutritional supplements $(2,3)$ & 1.494 & 0.887 & & n.q. & n.d. \\
\hline
\end{tabular}




\begin{tabular}{|c|c|c|c|c|c|}
\hline Types of food and processing & Genistein & Daidzein & Glycitein & Biochanin A & Formononetin \\
\hline Doughnuts $(2,8)$ & $17.81-32.15$ & $10.55-19.73$ & 0.363 & n.d. & n.d. -0.007 \\
\hline Energy bars (2) & 32.69 & 18.04 & & n.d. & n.d. \\
\hline English muffin (2) & 2.296 & 1.771 & & n.d. & n.d. \\
\hline Flour, granary (12) & 0.031 & 0.082 & & & \\
\hline Flour, wholemeal (11) & 0.146 & 0.024 & & & \\
\hline Fruit cake (1) & n.d. & n.d. & n.d. & n.d. & n.d. \\
\hline Potato, French fries (8) & 0.002 & 0.001 & 0.001 & & 0.003 \\
\hline Ginger cake (1) & n.d. & n.d. & n.d. & n.d. & n.d. \\
\hline Ginger root (1) & 0.3 & 0.1 & 0.1 & n.d. & n.d. \\
\hline Granola bar (8) & 0.039 & 0.017 & 0.001 & & 0.004 \\
\hline Ice cream roll (3) & 5.12 & 2.21 & 0.45 & n.d. & n.d. \\
\hline Marmite (1) & n.d. & n.d. & n.d. & n.d. & n.d. \\
\hline Mince, soy based (3) & 190.05 & 82.28 & 13.76 & n.d. & 0,08 \\
\hline Muesli (10) & $0.02-0.03$ & n.d. & n.d. & n.d. & 0.01 \\
\hline Muesli. extra fruit (10) & 30.65 & 16.93 & 0.13 & n.d. & 0.04 \\
\hline Black bean sauce ( 8 ) & 24.87 & 23.04 & 5.27 & & 0.017 \\
\hline Hamburger, vegetarian (3) & $33.09-40.11$ & $3.36-9.85$ & $0.65-1.14$ & n.d.-0.02 & n.d. -0.02 \\
\hline Hamburger, vegetarian $(2,8)$ & $11.11-20.2$ & $4.62-30.5$ & 0.812 & n.d. & 0.019 \\
\hline Honey (7) & n.d. & & & & \\
\hline Hummus (8) & 0.093 & 0.008 & 0.001 & & 0.034 \\
\hline Ice cream, soy based (3) & 80.43 & 36.07 & $17 \cdot 31$ & 0.5 & 0.56 \\
\hline Kala chana seed (9) & 6.4 & n.d. & & 12.6 & n.d. \\
\hline Lasagne (8) & 0.002 & 0.001 & 0.001 & & 0.003 \\
\hline Licorice, black (2) & 5.99 & 2.93 & & n.d. & 14.93 \\
\hline Miso paste $(4,8)$ & $59.02-672$ & $44.24-593$ & 8.00 & & 0.053 \\
\hline Miso soup $(2,8)$ & $4.37-10.10$ & $4.30-11.27$ & 0.276 & n.d. & $0.001-0.328$ \\
\hline Pancakes, waffles (2) & $13 \cdot 5^{8}$ & 13.00 & & n.d. & n.d. \\
\hline Pancakes (8) & 0.009 & 0.002 & 0.001 & & 0.008 \\
\hline Pasta (11) & n.d. -0.06 & n.d.-0.04 & n.d.-0.02 & n.d. -0.10 & n.d.-0.03 \\
\hline Peanut butter $(6,8)$ & n.d. -0.382 & n.d. -0.36 & $0.002-0.21$ & 0.01 & $0.007-0.03$ \\
\hline Pizza $(2,8)$ & $0.077-2.42$ & $0.065-2.31$ & 0.001 & n.d. & n.d. -0.002 \\
\hline Protein bar (8) & 19.13 & 7.89 & 0.007 & & 0.008 \\
\hline Quorn, mycoprotein, raw/fried (1) & n.d. & n.d. & n.d. & n.d. & n.d. \\
\hline Quorn, microprotein chicken style (3) & n.d. & n.d. & n.d. & 0.05 & n.d. \\
\hline Sago (11) & n.d. & 0.036 & & & \\
\hline Sausage, soy-based (3) & 25.96 & 11.02 & 2.38 & 0.05 & 0.05 \\
\hline Sausage, vegetarian $(1,3)$ & $0.05-27$ & Traces-22 & Traces-11 & n.d. -3 & n.q.--0.04 \\
\hline Semolina $(10,11)$ & n.q. -0.181 & n.d. -0.055 & n.d. & 0.07 & n.d. \\
\hline Sesame mochi (1) & n.d. & n.d. & 0.3 & n.d. & n.d. \\
\hline Soreen cake (1) & n.d. & n.d. & n.d. & n.d. & n.d. \\
\hline Soup, vegetable (8) & n.d. & n.d. & n.d. & & n.d. \\
\hline Soy bacon bits (8) & 48.97 & 9.98 & 1.00 & & 0.041 \\
\hline Tempeh (8) & 107 & 69.7 & 5.71 & & 0.019 \\
\hline Tofu $(2,8,9)$ & $122.8-171$ & $93 \cdot 4-113 \cdot 4$ & $7 \cdot 30$ & n.d. & n.d.-0.009 \\
\hline Trifle (3) & n.d. & n.d. & 0.01 & n.d. & n.d. \\
\hline Yogurt, soy-based $(3,8)$ & $65.65-69.31$ & $12.92-33.64$ & $0.12-2.97$ & n.d. & n.d. -0.015 \\
\hline
\end{tabular}

Note: $\quad$ n.d. = non-detectable; n.q. = non-quantifiable.

Source: (1) Clarke et al., 2013a; (2) Horn-Ross et al. 2000; (3) Kuhnle et al., 2008a; (4) Liggins et al., 200ob; (5) Mazur et al., 1996; (6) Kuhnle et al., 2008b; (7) Sergiel et al., 2014; (8) Thompson et al., 2006; (9) Frank et al., 1994 (10) Kuhnle et al., 2009a; (11) Liggins et al., 2002. 
Table 21: Isoflavone content ( $\mathrm{mg} / \mathrm{kg} \mathrm{dw}$ ) in other types of food

\begin{tabular}{|c|c|c|c|c|c|}
\hline Types of food and processing & Genistein & Daidzein & Glycitein & Biochanin A & Formononetin \\
\hline Bread, various types (2) & n.q. -0.105 & n.q. -0.131 & & n.d.-n.q. & n.q. -0.029 \\
\hline Caraway seed (5) & 0.08 & n.d. & & & \\
\hline Granola candy bar (2) & 0.783 & 0.519 & & 0.022 & 0.041 \\
\hline "Japanese pagoda tree" (Sophora japonica) (3) & 2.65 & 3.19 & & 8.3 & 3.22 \\
\hline Poppy seed (5) & 0.07 & 0.18 & & & \\
\hline Soy meal, defatted (4) & 744 & 625 & & & n.d. \\
\hline Soy paste, sweet (4) & $5 \cdot 3$ & 51.3 & & & \\
\hline Soy paste, hot (4) & 40.5 & 106.9 & & & n.d. \\
\hline Tofu, various products $(4,6)$ & $128.1-1321.3$ & $115 \cdot 9-1228.8$ & $66.3-118.7$ & & n.d. \\
\hline Trifle (1) & n.d. & n.d. & 0.01 & n.d. & n.d. \\
\hline
\end{tabular}

Note: $\quad$ n.d. = non-detectable; n.q. = non-quantifiable.

Source: (1) Kuhnle et al., 2008a; (2) Mazur et al., 1996; (3) Mazur et al., 1998b; (4) Wang et al., 1990; (5) Mazur \& Adlercreutz, 1998; (6) Song et al., 1998.

Liggins et al. (200ob) compared the isoflavone profile and constituent levels in butterbeans, common beans, French beans, haricot beans (fresh common bean), mung bean, peas and runner beans with the profile and constituent levels in the corresponding cooked beans. Although cooking influenced the different types of bean to a different extent, the general trend was a reduction in the level of the various isoflavones by $10-60 \%$.

\subsubsection{Isoflavones in dietary supplements}

Two research teams have determined the phytoestrogen content of dietary supplements: Clarke et al. (2008) used LC-MS/MS and Penalvo et al. (2004) GC-MS. Penalvo et al. (2004) studied seven isoflavone concentrates in the form of tablets or capsules from Finland, Israel, and the USA, as well as seven soy protein-based products in the same form from Finland, France, the Netherlands, Norway, or the USA. The total isoflavone content per piece (tablet or capsule) of the isoflavone concentrates was from 4.3 to $28.4 \mathrm{mg}$, which was $-45 \%$ to $9 \%$ of the quantity stated on the product label. In soy protein-based products, the total isoflavone content per piece was 0.1 to $30.5 \mathrm{mg}$, which was between $-76 \%$ and $-0.7 \%$ of the quantity stated on the product. Across all products, the level of daidzein was between 0.03 and $21.5 \mathrm{~g} / \mathrm{kg}$, the level of genistein between 0.03 and $54.1 \mathrm{~g} / \mathrm{kg}$, and the level of glycitein between 0.1 and $7.0 \mathrm{~g} / \mathrm{kg}$ when the corresponding amounts in soybean seeds were on average 0.39 (range: $0.19-0.59$ ), 0.49 (range: $0.28-0.70$ ), and $0.04(0.02-0.08) \mathrm{g} / \mathrm{kg}$ for daidzein, genistein, and glycitein, respectively. It was also noted that in the preparation of the isoflavone-rich products, lignans were found present at considerable quantities. Therefore, when making exposure assessment of phytoestrogens from isoflavone food supplements, the contribution from lignans needs to be considered. 
Clarke et al. (2008) undertook to quantify the concentrations of ten common isoflavones in thirty-five dietary supplement samples on sale in the UK, Canada, and Italy for management of menopause symptoms. Soya isoflavones were present in all products. Moreover, the majority also contained red clover isoflavones (biochanin A and formononetin) and some of the Kudzu isoflavones puerarin (and daidzein). The content of total isoflavones per dose ranged from $<1$ to $53 \mathrm{mg}$. Trace amounts of coumestrol was found in six products. Only fourteen of the thirty-five supplements were found to deliver more than or equal to $40 \mathrm{mg}$ of aglycone isoflavones/day, a consensus dose value recognised as delivering therapeutic benefit. Both studies point to the difficulty of consumers to know that they receive the amount of total isoflavones from the supplement they are targeting.

\subsubsection{Influence of storage and processing on isoflavone content}

Lee et al. (2003b) performed an extensive study to evaluate how the isoflavone profile and content of soybeans were influenced during storage up to three years. The studies showed (1) there was a slight reduction in total isoflavone content in seeds stored for one, two, or three years; (2) the concentration of the malonylglucoside isoflavones decreased during storage (at least to half within three years, with the magnitude of the decrease similar for the daidzin and genistin forms); and (3) the concentration of glucosides and aglycons increased during storage (2-4 times during two years), glycosides always occurring at much higher levels than aglycones. Because high temperatures have been shown to degrade malonylglucoside forms in solution and because the temperature during storage was not perfectly controlled, it is possible that the degradation observed could have been induced by climatic conditions.

Up to the early 1990s, isoflavones had been extracted into hot aqueous organic solvents and found to be the unconjugated aglycones (Eldridge \& Kwolek, 1983). However, in 1991, Kudou et al. (1991) noted that a different set of compounds was extracted if the extraction took place at lower temperatures when a large portion of the extracted compounds were 6 "-O-malonyl- $\beta$-glucoside conjugates of the aglycones. Shortly thereafter, it became clear that most foods containing isoflavones have a mixture of $\beta$-glucosides 6"-O-malonyl- $\beta$-glucoside conjugates and 6"-O-acetyl$\beta$-glucoside conjugates (Barnes et al., 1994; Wang \& Murphy, 1994a, 1994b). Against this historical background, it seems obvious to ask the question: How will various types of processing of the harvested crop and food processing influence the isoflavone profile of the food?

As shown in Tables 8 and 9, defattening of oil-rich crops (such as soybeans and peanuts) increases the total isoflavone content.

Coward et al. (1998) set out to determine to what extent cooking of soy foods alters the isoflavone glucoside conjugate composition and whether the various isoflavone moieties are stable under common cooking conditions. Defatted soy flour that had not been heat treated during isoflavone extraction consisted mostly of 6"-Omalonyl- $\beta$-glucoside conjugates. In contrast, toasted soy flour contained large

amounts of $6^{\prime \prime}$-O-acetyl- $\beta$-glucoside conjugates, formed by heat-induced 
decarboxylation of the malonate group to acetate. Soymilk and tofu consisted almost entirely of $\beta$-glucoside conjugates; low fat versions of these products were markedly depleted in isoflavones. Alcohol-washed soy-protein concentrates contained few isoflavones. Isolated soy protein and textured vegetable protein consisted of a mixture of all 3 types of isoflavone conjugate. Baking or frying of textured vegetable protein at $190^{\circ} \mathrm{C}$ and baking of soy flour-based cookies did not alter total isoflavone content, but there was a steady increase in $\beta$-glucoside conjugates at the expense of $6^{\prime \prime}$-O-malonyl- $\beta$-glucoside conjugates.

Grün et al. (2001) heated tofu in water at $80-100{ }^{\circ} \mathrm{C}$ for $0-40$ minutes and quantified the various isoflavones in the processed product. There was a decrease in total isoflavone content, most likely due to leaching of isoflavones into the water. Daidzein conjugates were much more affected than genistein conjugates. Changes in the profile of the daidzein series suggest little decarboxylation of the malonylglycoside to the acetylglycoside, but considerable de-esterification of the malonyl- and acetylglycoside to the $\beta$-glucoside. A strong temperature-dependent decrease in daidzin suggests possible thermal degradation of daidzein in addition to losses due to leaching (Grün et al., 2001). Xu et al. (2002) reported the order of thermal stabilities of the purified isoflavone glucosides, from lowest to highest, as glycitin, genistin, and daidzin.

In a similar study, Eisen et al. (2003) examined the stability of genistein and daidzein derivatives in soy milk. Storage at elevated temperatures reduced genistin content. In early stages at elevated temperatures the 6"-O-acetyldaidzin concentration increased, followed by a slow decrease.

Isoflavones occur primarily as glycosides (namely malonyl-, acetyl-, and nonconjugated $\beta$-glycosides) and a rather small percentage as the bioactive aglycons. The different chemical structures of isoflavones can dictate their stability during processing. To investigate this possibility, Mathias et al. (2006) determined the effects of $\mathrm{pH}$ and thermal treatments on conjugated isoflavones for interconversions and loss. When heated at 25,80 , and $100{ }^{\circ} \mathrm{C}$ under neutral, acidic, and basic conditions, conjugated daidzin and genistin interconverted and some loss in total isoflavone derivatives was noted for each isoflavone, especially under elevated $\mathrm{pH}$ and temperature. The same observation was made by Unger et al. (2003), whereas Stintzing noted the largest decay at lower pH (3.1). Malonylglycosides were more stable than acetylglycosides, especially under acidic conditions. Overall loss in isoflavone derivatives was higher for daidzin than for genistin glycosidic forms (Mathias et al., 2006; Stintzing et al., 2006).

Because heat processing of soybeans has the potential to influence the content and distribution profile of isoflavones, Huang and Chou (2008) steamed black soybeans and black soybean koji after heating at $80{ }^{\circ} \mathrm{C}$ or higher for thirty minutes. In another study, Xu and Chang (2008a) boiled, pressure boiled, steamed, and pressure steamed yellow and black soybeans. All these processing methods, except pressure steaming, reduced the total isoflavone content. The soybean products showed reduced contents of malonylglucoside, acetylglucoside, and aglycone isoflavone and an increased content of $\beta$-glucosids. After a temperature of $100{ }^{\circ} \mathrm{C}$ in the study of 
Huang and Chou (2008), steamed black soybean retained about $90 \%$ of its original malonylglucoside isoflavone and $84 \%$ of its original acetylglucoside isoflavone compared with lower residuals of about $81 \%$ (malonylglucoside isoflavone) and $79 \%$ (acetylglucoside isoflavone) for black soybean koji. In contrast, the heated black soybeans showed an aglycone residual of $68 \%$, which is less than the $80 \%$ noted with the heated black soybean koji.

In agreement with these observations, Barnes et al. (1994) reported that, whereas soybeans and defatted soy flour (which had been minimally heated during preparation) contained mostly isoflavone 6"-malonyl-glucoside conjugates, with lesser quantities of the $\beta$-glucosides and only trace amounts of $6^{\prime \prime}$-O-acetylglucoside conjugates, soy milk, tofu, and soy molasses, each of which involves heating to $100^{\circ} \mathrm{C}$ during their manufacture, contained mostly isoflavone $\beta$-glucosides. Toasted soy flour and isolated soy protein contained moderate amounts of each of the isoflavone conjugates.

Pods of immature soybeans are steamed or boiled, seasoned with salt or spices, and served as hors d'oeuvre (edamame). Simonnne et al. (2000) compared the retention and changes of soy isoflavones during processing of immature and mature soybeans. In most varieties, the total isoflavone content (mainly in the form of malonyl isoflavones but also some glucoside isoflavones) was higher in immature soybeans than in mature soybeans but there were exceptions. When retention of isoflavones during processing was studied in one soybean variety, it was demonstrated that the mean total isoflavone retention in immature soybeans was $46 \%$ (boiling), 53\% (freezing), and 40\% (freeze-drying). Boiling resulted in both loss of total isoflavones (in part due to leaching) and in a substantial increase in daidzin, genistin and glycitin.

Wang and Murphy (1994a, 1994b) reported non-fermented soybean foods to have most isoflavones as isoflavone glucosides, whereas fermented foods contained greater levels of aglycones. In peanuts, Chukwumah et al. (2007b) noted boiling to have a larger influence on total isoflavones than roasting, i.e. boiled peanuts had the highest total flavonoid content. In particular, the biochanin A and genistein content of boiled peanut extracts were two- and fourfold higher, respectively, than in the raw peanut.

\subsection{Lignan levels in foods}

Lignans are non-nutritious secondary metabolites found in a myriad of plants. They can occur as free aglycones or as glycosides stored in vacuoles. Lignans are present in various plant parts, including leaves, stems, flowers, and seed coats (DellaGreca et al., 2013). In cereals and grains, lignans are mostly found in the outer layer of the hull. In berries, they are commonly located in the seeds (Dixon, 2004; Peterson et al., 2010). Their primary function is to serve as the plant's defence against different diseases and pests (DellaGreca et al., 2013).

The most commonly reported lignans are secoisolariciresinol and matairesinol. However, lariciresinol, syringaresinol, pinoresinol, and medioresinol are reported in some foodstuffs. The molecular structure of lignans commonly occurring in foods is 
shown in Figure 4. The estimated lignan intake in five European countries (Denmark, Finland, Italy, Sweden, and the United Kingdom) was calculated using the Dutch lignan database based on four lignans: secoisolariciresinol, matairesinol, lariciresinol, and pinoresinol (Tetens et al., 2013). Food consumption data were extracted from cohort studies performed in Sweden and national dietary surveys in the other countries. The total estimated lignan intake was from 1.08 to $1.77 \mathrm{~g} /$ day, with the lowest estimated intake being in Finland and the highest in Sweden. Similar intakes were observed in men and women in most countries. However, Swedish men had a higher daily estimated intake of around $0.4 \mathrm{mg}$ compared with women. Although secoisolariciresinol and matairesinol are the most commonly reported lignans, together they only accounted for about $13.8-24.3 \%$ of the total estimated lignans in the diet. Lariciresinol and pinoresinol accounted for the bulk of the estimated lignan intake.

As mentioned in a previous chapter, a solvent such as methanol is commonly used to extract the lignans from the food sample. To establish the true lignan content of foods it is necessary to free the lignans bound to sugar molecules by hydrolysis. This can be achieved by means of acids, bases, or enzymes. Several extraction methods have been applied to maximise the lignan yield. However, some lignans are acid and base labile. Therefore, the use of a concentrated acid or base can cause lignans to be destroyed or transformed into other lignans, skewing the content analysis (Smeds et al., 2007; Schwartz et al., 2009). One such transformation that occurs during acidic conditions is the transformation of secoisolariciresinol to anhydrosecoisolariciresinol. If the sample were not analysed for anhydrosecoisolariciresinol, the original content of secoisolariciresinol in the sample will be underestimated (Smeds et al., 2007). 
Figure 4: The molecular structure of lignans (A) matairesinol, (B) secoisolariciresinol, (C) lariciresinol, (D) pinoresinol, $(E)$ medioresinol, and $(F)$ syringaresinol

(A)<smiles>COc1cc(CC2COC(=O)C2Cc2ccc(O)c(OC)c2)ccc1O</smiles>

(C)<smiles>COc1cc(CC2COC(c3ccc(O)c(OC)c3)C2CO)ccc1O</smiles>

(E)<smiles>COc1cc(C2OCC3(c4cc(OC)c(O)c(OC)c4)COCC23)ccc1O</smiles>

(B)<smiles>COc1cc(CC(CO)C(CO)Cc2ccc(O)c(OC)c2)ccc1O</smiles>

(D)<smiles>COc1cc(C2OCC3(c4ccc(O)c(OC)c4)COCC23)ccc1O</smiles>

(F)<smiles>COc1cc(C2OCC3(c4cc(OC)c(O)c(OC)c4)COCC23)cc(OC)c1O</smiles> 
The different extraction methodologies can lead to varying extraction yields of lignans, which must be considered when comparing the data from various studies. This can only partly explain the wide ranges in the content of the various lignans in foodstuffs that have been reported between varieties of the same species. Differences between genotypes is likely to be as big a contributor to the different lignan yields as the environmental conditions during cultivation and extraction methods applied.

\subsubsection{Lignans in animal products}

\section{Seafood}

Only a handful of publications have reported on the level of the lignans matairesinol and secoisolariciresinol in seafood. Low levels of matairesinol were found in mussels (0.02 mg/kg fw) and prawns ( $0.03 \mathrm{mg} / \mathrm{kg} \mathrm{fw}$ ) (Kuhnle et al., 2008a). No secoisolariciresinol was found in mussels and non-quantifiable levels were detected in prawns. Among fishes, salmon and cod were investigated for the same compounds but only trace levels of matairesinol were found. In a study on canned tuna, on the other hand, very low quantities of four lignans were detected: matairesinol ( 0.001 $\mathrm{mg} / \mathrm{kg} \mathrm{fw}$ ), pinoresinol (0.002 mg/kg fw), lariciresinol (0.005 mg/kg fw) and secoisolariciresinol (0.002 mg/kg fw) (Thompson et al., 2006). Kuhnle et al. (2008a) investigated the levels of lignans in the brine of canned tuna, but only nonquantifiable levels of secoisolariciresinol were found. Clarke et al. (2013a) reported 1 $\mathrm{mg}$ secoisolariciresinol/kg fw of sushi but did not state which fish was used. In short, seafood represents a negligible contribution to the total lignin intake in consumers.

\section{Dairy products}

To analyse lignans, Kuhnle et al. (2008a) dissolved dairy products in methanol/sodium acetate, supplied the $\beta$-glucuronidase, cellulase, and $\beta$-glucosidase and extracted the compounds using solid phase methodology. Extracts were injected into a HPLC that separated the lignans and quantified them by MS/MS. The levels of matairesinol and secoisolariciresinol in dairy products from cow's milk are shown in Table 22. Most of the analysed dairy products contained low levels of these constituents. The occurrence of lignans in dairy products is likely due to cows eating feedstuffs containing this class of compounds. The occurrence of two other lignans, pinoresinol and lariciresinol, was investigated using a similar chemical analytical method, which demonstrated that also these occurred at low levels (pinoresinol, $0.002 \mathrm{mg} / \mathrm{kg}$ fw and lariciresinol $0.003 \mathrm{mg} / \mathrm{kg} \mathrm{fw}$ ) (Thompson et al. 2006).

A sample of milk powder was shown to contain $0.04-0.11 \mathrm{mg}$ matairesinol $/ \mathrm{kg} \mathrm{fw}$ and $0.03-0.04 \mathrm{mg}$ secoisolariciresinol $/ \mathrm{kg} \mathrm{fw}$, which is about double the amount of matairesinol in milk. A non-dairy ice cream was shown to contain similar levels of matairesinol and secoisolariciresinol as ice cream produced from cream (0.03 mg matairesinol $/ \mathrm{kg} \mathrm{fw}$ and trace levels of secoisolariciresinol) (Kuhnle et al., 2008a).

Only trace levels of matairesinol and secoisolariciresinol were identified in a single sample of goat's milk (Kuhnle et al., 2008a). 
Table 22: Lignan content ( $\mathrm{mg} / \mathrm{kg}$ fresh weight) in dairy products from bovine milk

\begin{tabular}{lll} 
Product & Secoisolariciresinol & Matairesinol \\
Milk & n.q. -0.05 & n.q. -0.05 \\
Cream & n.q.-0.01 & 0.02 \\
Yoghurt, natural and flavoured & n.q. -0.44 & n.q. -0.02 \\
Butter & n.d.-0.01 & $0.01-0.05$ \\
Crème fraiche & 0.02 & 0.03 \\
Cheese & n.d.-0.25 & n.d.-0.15 \\
Cottage cheese & n.d.-n.q. & n.q.-0.04 \\
Soft cheese, Philadelphia type & $0.01-0.12$ & n.q. \\
Ice cream & n.d.-0.02 & n.q.-0.06 \\
Ice cream dessert & n.q. 0.03 & n.q.-0.05 \\
\hline
\end{tabular}

Note: $\quad$ n.d. = non-detectable; n.q. = non-quantifiable.

Source: Thompson et al., 2006; Kuhnle et al., 2008a.

Poultry and egg

Only limited information is available on the lignan content of poultry products. Roasted chicken contained low levels of matairesinol (0.01-0.02 mg/kg fw) and trace levels of secoisolariciresinol (Kuhnle et al., 2008a). Comparable levels have been found in chicken skin. No data on the lignan content in fresh chicken meat were found. Eggs contain non-detectable to low levels $(0.02 \mathrm{mg} / \mathrm{kg} \mathrm{fw})$ of both matairesinol and secoisolariciresinol (Horn-Ross et al., 2000; Kuhnle et al., 2008a).

\section{Meat products}

Kuhnle and co-workers (2008a) reported on the level of lignans in roasted and grilled meat from cow, pig, and lamb. Roasted beef contains $0.05-0.15 \mathrm{mg}$ matairesinol $/ \mathrm{kg}$ $\mathrm{fw}$, roasted lamb $0.03 \mathrm{mg}$ matairesinol $/ \mathrm{kg} \mathrm{fw}$, and roasted pork $0.02-0.03 \mathrm{mg}$ matairesinol $/ \mathrm{kg} \mathrm{fw}$ and up to $0.02 \mathrm{mg}$ secoisolariciresinol $/ \mathrm{kg} \mathrm{fw}$. Roasted beef and lamb contained trace levels of secoisolariciresinol. Higher levels of these two lignans have been reported in grilled liver of lamb and pig (Kuhnle et al., 2008a). In contrast to roasted beef, corned beef was reported to contain only trace levels of matairesinol but $0.04 \mathrm{mg}$ secoisolariciresinol/kg fw (Kuhnle et al., 2008a).

\subsubsection{Lignans in beverages}

The lignan content in beverages not containing dairy ingredients are summarised in Table 23. In addition to the four reported lignans (secoisolariciresinol, lariciresinol, matairesinol, and pinoresinol), red and white wine also contain the lignans isolariciresinol and syringaresinol. The levels in red wine were $0.54-0.92 \mathrm{mg} / \mathrm{kg} \mathrm{fw}$ of isolariciresinol and $0.015-0.11 \mathrm{mg} / \mathrm{kg} \mathrm{fw}$ of syringaresinol. White wine contained $0.26-0.46 \mathrm{mg}$ isolariciresinol $/ \mathrm{kg}$ fw and $0.01-0.02 \mathrm{mg}$ syringaresinol $/ \mathrm{kg} \mathrm{fw}$. Nurmi et al. (2003) reported only small variations in lignan content between wines. White wines were reported to contain slightly lower levels of secoisolariciresinol and total lignan levels, possibly because of differences in the winemaking process, as well as in storage conditions and times. If wine were stored in wood barrels, there may be a release of a number of lignans from the wood, with the amount released depending on the time in storage. 
Table 23: Lignan content ( $\mathrm{mg} / \mathrm{kg}_{\text {fresh weight)1 }}$ in different beverages

\begin{tabular}{|c|c|c|c|c|}
\hline Product & Secoisola-riciresinol & Lariciresinol & Matai-resinol & Pino-resinol \\
\hline Soya milk & 0.011 & 0.066 & n.d. & 0.300 \\
\hline Coffee & n.d. -8.26 & $0.009-0.131$ & n.d. -0.58 & $0.001-0.015$ \\
\hline Tea, green & $0.104-0.45$ & $0.001-0.187$ & $0.001-0.05$ & $0.014-0.057$ \\
\hline Tea, black & $0.038-0.09$ & $0.002-0.308$ & $0.001-0.02$ & $0.04-0.406$ \\
\hline Chocolate milk & n.d. & 0.009 & n.d. & 0.013 \\
\hline Cola & n.d. & n.d. & n.d. & n.d. \\
\hline Tomato juice & $0.016-0.054$ & $0.021-0.097$ & n.d. -0.003 & $0.01-0.099$ \\
\hline Orange juice & $0.027-0.08$ & $0.002-0.074$ & n.d. & $0.001-0.075$ \\
\hline Grapefruit juice & $0.06-0.067$ & $0.05-0.051$ & n.d. & $0.033-0.048$ \\
\hline Grape juice & $0.025-0.108$ & $0.033-0.065$ & n.d. & $0.007-0.037$ \\
\hline Cider & 0.48 & & n.d. & \\
\hline Beer & n.d. -0.63 & $0.006-0.092$ & n.d.-0.011 & $0.003-0.22$ \\
\hline Red wine & $0.178-0.613$ & $0.024-0.169$ & n.d. -0.2 & $0.004-0.119$ \\
\hline White wine & $0.038-0.122$ & $0.016-0.119$ & $0.004-0.05$ & $0.001-0.03$ \\
\hline Sherry & $0.16-0.38$ & & n.d. -0.15 & \\
\hline Whiskey & 0.04 & & n.d. & \\
\hline Gin & n.d. & & n.d. & \\
\hline
\end{tabular}

Note: $\quad{ }^{1} 1 \mathrm{mg} / \mathrm{l}$ was assumed to correspond to $1 \mathrm{mg} / \mathrm{kg}$. n.d. = non-detectable.

Source: Horn-Ross et al., 2000; Nurmi et al., 2003; Milder et al., 20051; Thompson et al., 2006; Kuhnle et al., 2008b; Clarke et al., $2013 a$.

Lignan levels in beverages are quite low, except for secoisolariciresinol and to some extent matairesinol in coffee. The highest level of secoisolariciresinol reported in coffee was almost 20 times higher than that reported in cider, but it should be noted that different investigators have reported quite different levels in coffee. Matairesinol was found in very few products in comparison with secoisolariciresinol, which was detected in almost all products.

Powdered coffee of five brands were soaked in water at room temperature overnight and the solution hydrolysed and extracted in two steps before derivatisation and analysis by GC-MS. Of the analysed lignans, only secoisolariciresinol was detected at levels between 3.93 and $7.14 \mathrm{mg} / \mathrm{kg} \mathrm{dw}$ (Mazur et al., 1998a). One sample of instant coffee contained $7.16 \mathrm{mg}$ secoisolariciresinol $/ \mathrm{kg} \mathrm{dw}$.

Brewed teas (boiling water poured over tea leaves and kept in a water bath at 90$95{ }^{\circ} \mathrm{C}$ for 10 minutes) prepared from green or fermented tea from China, India, Indonesia, Japan, Russia, Sri Lanka, and Taiwan contain 6.09-28.87 mg secoisolariciresinol $/ \mathrm{kg} \mathrm{dw}$ and $0.56-3.05 \mathrm{mg}$ matairesinol $/ \mathrm{kg} \mathrm{dw}$ (Mazur et al., 1998a) Very similar data were obtained when tea leaves were soaked in water at room temperature overnight before being extracted for chemical analysis. Teas prepared from green tea leaves were in the upper part of the reported ranges.

A cocktail having cranberry as an ingredient was reported to contain secoisolariciresinol ( $0.036 \mathrm{mg} / \mathrm{kg} \mathrm{fw}$ ), lariciresinol (0.021 mg/kg fw), and pinoresinol (0.002 $\mathrm{mg} / \mathrm{kg} \mathrm{fw})$, but no matairesinol was detected (Thompson et al., 2006). 


\subsubsection{Lignans in fruits and berries}

The content of lignans in fruits and berries are reported in Tables $24-26$. Because levels have been reported both in $\mathrm{fw}$ and in $\mathrm{dw}$ and because enough information is not always available to recalculate the data into $d w$, data on the content of lignans in fruits given on a fw basis is reported in Table 24 and data given on a dw basis in Table 25. The data on lignan content in berries is given on a dw basis in Table 26.

As shown in Tables 24 and Table 25, the lignan content in fruits is generally low. Highest levels are found in bitter orange, orange, kiwi, and pear. As shown in Table 25, fruits generally contain low levels of matairesinol but 10- to 200-fold higher amounts of secoisolariciresinol, the exception being grapes, which had comparable levels of these lignans. Apricot, nectarine, and plum were also analysed for the presence of lariciresinol and pinoresinol (Thompson et al., 2006). The levels of lariciresinol were $7.5,3.75$, and $0.29 \mathrm{mg} / \mathrm{kg} \mathrm{dw}$ in apricot, nectarine, and plum, respectively, and the levels of pinoresinol $22.4,11.9$ and $5.3 \mathrm{mg} / \mathrm{kg} \mathrm{dw}$, respectively. Apparently, the latter two lignans are the most common lignans in fruits.

Table 24: Lignan content ( $\mathrm{mg} / \mathrm{kg}$ fresh weight) in fruit

\begin{tabular}{|c|c|c|c|c|c|c|}
\hline Fruit & Secoisolariciresinol & Lariciresinol & Matairesinol & Syringaresinol & Pinoresinol & Medioresinol \\
\hline Apple & n.d.-0.003 & $0.01-0.55$ & n.d.-0.03 & n.d. & n.d.-0.008 & n.d. \\
\hline Banana & n.d. -0.006 & n.d.-0.17 & n.d. -0.001 & 0.19 & n.d.- -0.002 & n.d. \\
\hline Cherries & 0.06 & 0.41 & n.d. & & 1 & \\
\hline Grapefruit & n.d.-o.og & $0.029-0.95$ & n.d.-0.02 & & $0.004-0.45$ & \\
\hline Grape & $0.016-0.32$ & $0.062-0.52$ & n.d. -0.05 & 0.21 & n.d.- -0.28 & 0.08 \\
\hline Kiwi & $1.06-1.16$ & $0.1-0.2$ & n.d. & n.d.-0.08 & n.d. -0.13 & $0.04-0.05$ \\
\hline Lemon & 0.04 & 0.25 & n.d. & 0.57 & 1.85 & 0.64 \\
\hline Mandarin & 0.03 & 0.57 & 0.01 & & 0.21 & \\
\hline Melon, cantaloupe & 0.047 & 0.018 & n.q. & & 0.003 & \\
\hline Melon, Galia & 0.05 & 0.44 & n.d. & & 0.22 & \\
\hline Olives & $0.07-0.306$ & $0.006-0.36$ & n.d.-0.001 & & $0.019-0.37$ & \\
\hline Orange & $0.025-0.56$ & $0.092-1.93$ & n.d.-0.02 & $0.48-2.02$ & $0.031-0.51$ & $0.06-0.19$ \\
\hline Orange, bitter & 0.26 & 1.92 & n.d. & 2.93 & 6.54 & 1.25 \\
\hline Peach & $0.11-0.279$ & $0.095-0.8$ & n.d.-0.016 & n.d.-0.03 & $0.371-1.86$ & $0.02-0.05$ \\
\hline Pear & $0.02-0.04$ & $0.34-1.55$ & n.d. & 0.14 & $0.02-0.34$ & 0.05 \\
\hline Japanese pear & 0.07 & 0.21 & n.d. & 0.01 & n.d. & 0.01 \\
\hline Pineapple & 0.07 & $0.24-0.67$ & $0.1-0.18$ & $0.81-0.92$ & $0.03-0.04$ & $0.01-0.03$ \\
\hline Japanese plum & 0.16 & 0.2 & n.d. & n.d. & 0.42 & 0.01 \\
\hline Prune & $0.75^{8}$ & & n.d. & & & \\
\hline Watermelon & $0.014-0.23$ & $0.011-0.69$ & n.d.-n.q. & $0.01-0.42$ & $0.003-0.12$ & n.d.-0.02 \\
\hline
\end{tabular}

Note: n.d. = non-detectable; n.q. = non-quantifiable.

Source: Horn-Ross et al., 2000; Milder et al., 2005; Thompson et al., 2006; Penalvo et al., 2005, 2008; Clarke et al., 2013a. 
Table 25: Lignan content ( $\mathrm{mg} / \mathrm{kg}$ dry weight) in fruit

\begin{tabular}{lll} 
Fruit & Secoisolariciresinol & Matairesinol \\
Apple & n.q.-0.21 & n.d.-0.0375 \\
Apricot ${ }^{1}$ & $2.2-4.8$ & n.d.-0.02 \\
Banana & $0.01-0.1$ & n.d.-0.02 \\
Cantaloupe melon & 1.99 & 0.02 \\
Grapes & $0.33-0.43$ & $0.34-0.50$ \\
Grapefruit & 2.48 & 0.03 \\
Mango & 0.85 & 0.08 \\
Nectarine ${ }^{2}$ & $1.64-2.54$ & n.d. \\
Orange & 1.51 & 0.01 \\
Peach & 3.12 & 0.01 \\
Pear & $0.12-0.41$ & $0.01-0.0686$ \\
Plum & $0.05-5.89$ & n.d.-0.06 \\
Sharon & 1.45 & n.d. \\
Water melon & 4.37 & n.q. \\
\hline \multirow{2}{*}{${ }^{1}$ recalculated, assuming moisture content of $86 \%$} & \\
${ }^{2}$ recalculated, assuming moisture content of $89 \%$. & \\
$\quad$ n.d. = non-detectable; n.q. = non-quantifiable. &
\end{tabular}

Source: Mazur and Adlercreutz, 1998; Milder et al., 2005; Kuhnle et al., 2007, 2009b; Smeds et al., 2012.

Kuhnle et al. (2009b) examined the variability in lignan content in fruit from different countries. Although large variations were observed, no clear effect of country of origin or botanical type typical for the region could be detected. It was suggested that the variation observed could be due to different plant varieties grown at different locations in different soils.

In addition to the data on fruit in Tables 24 and 25, dried raisins, apricots, dates, and prunes were analysed for lignan content (Horn-Ross et al., 2000; Milder et al., 2005; Thompson et al., 2006). On a fw basis, one kilogram of raisins contained 0.08 $0.115 \mathrm{mg}$ secoisolariciresinol, $0.092-1.53 \mathrm{mg}$ lariciresinol, $0.004-0.522 \mathrm{mg}$ matairesinol, and n.d.-0.008 mg pinoresinol. Dried apricots analysed for the same lignans contained 1.53-3.3, 0.62, n.d.-0.006, and $1.9 \mathrm{mg} / \mathrm{kg}$ fw. Dried dates contained $1.062 \mathrm{mg}$ secoisolariciresinol, $1.16 \mathrm{~g} \mathrm{mg}$ lariciresinol, $0.003 \mathrm{mg}$ matairesinol, and 1.002 $\mathrm{mg}$ pinoresinol per $\mathrm{kg} \mathrm{fw}$. Lastly, dried prunes contained $1.038 \mathrm{mg}$ secoisolariciresinol, $0.021 \mathrm{mg}$ lariciresinol, $0.002 \mathrm{mg}$ matairesinol, and $0.715 \mathrm{mg}$ pinoresinol per $\mathrm{kg}$ fw.

Berries contain higher levels of secoisolariciresinol than fruits, with the highest levels reported in blackberries. Cloudberries have the highest lariciresinol content $(50.08 \mathrm{mg} / \mathrm{kg} \mathrm{dw})$ of all berries, the levels being between 4 and 50 times higher than in other analysed berries (Table 26). Smeds et al. (2012) compared the lignan content of bilberry, blackberry, black currant, cloudberry, cranberry, raspberry, red gooseberry, and sea buckthorn with the content in the respective seeds. For all eleven analysed lignans, seeds usually contained higher amounts of lignans than the whole berries (for compounds, see Table 23 and Smeds et al. 2012). The magnitude of the differences was from a few per cent up to more than $1,300 \%$ and depended on type of berry and which lignan constituent was studied. The berries listed in Table 26 were also analysed for their 7-hydroxymatairesinol content. Only strawberry was found to contain 7hydroxymatairesinol (0.095 mg/kg dw) (Smeds et al., 2012). 
Table 26: Lignan content ( $\mathrm{mg} / \mathrm{kg}$ dry weight) in various berries

\begin{tabular}{|c|c|c|c|c|c|c|}
\hline Berry & $\begin{array}{l}\text { Secoisolarici- } \\
\text { resinol }\end{array}$ & $\begin{array}{l}\text { Larici- } \\
\text { resinol }\end{array}$ & Matairesinol & $\begin{array}{l}\text { Syringa- } \\
\text { resinol }\end{array}$ & Pinoresinol & $\begin{array}{l}\text { Medio- } \\
\text { resinol }\end{array}$ \\
\hline Bilberry & 4 & 2.89 & n.d. & 8.01 & 0.623 & 5.48 \\
\hline Blackberry & $29.02-37.18^{1}$ & 13.19 & $0.037-0.225$ & 16.18 & 11.99 & 4.76 \\
\hline Blackcurrant & $3.88-5.63$ & 1 & n.d. -0.1 & n.d. & 0.42 & 1.03 \\
\hline Blueberry & 8.35 & & n.d. & & & \\
\hline Cloudberry & $2.03-4.1$ & 50.08 & n.d. & 32.1 & 3.84 & 37.55 \\
\hline Cranberry & $10.54-13.23$ & 3.26 & n.d. & 25.78 & 2.16 & 12.53 \\
\hline Gooseberry & 7.2 & & n.q. & & & \\
\hline Lingonberry & $15.1-23.2$ & 2.44 & n.d. & 9.05 & 10.73 & 14.43 \\
\hline Raspberry & $1.39-1.82$ & 4.06 & n.d. -0.03 & 3.88 & 3.77 & n.d. \\
\hline Red gooseberry & 2.64 & 0.897 & 0.074 & 4.98 & 0.294 & 0.674 \\
\hline Redcurrant & 1.65 & & n.d. & & & \\
\hline Sea buckthorn & 0.258 & 1.8 & n.d. & 11.77 & n.d. & 2.16 \\
\hline Strawberry & $0.73^{-15} .05^{1}$ & 11.73 & n.d. -0.05 & 5.86 & 14.03 & 6.14 \\
\hline
\end{tabular}

Note: $\quad{ }^{1}$ secoisolariciresinol reported as sum of anhydrosecoisolariciresinol and secoisolariciresinol (Mazur et al., 2000).

n.d. = non-detectable; n.q. = non-quantifiable.

Source: Mazur \& Adlercreutz, 1998; Mazur et al., 2000; Kuhnle et al., 2007; Smeds et al., 2012.

Yang et al. (2006) reported higher levels of secoisolariciresinol and matairesinol in sea buckthorn harvested between late August and early October compared with berries harvested in late October and November the same year. The differences were thought to be due to differences in maturity, but owing to the low number of samples, the possibility that the observed differences may be caused by natural variation could not be ruled out. An alternative explanation might be that the plants have a lower requirement of constituents contributing to plant defence late in the season.

In addition to the data on berries presented on a dw basis in Table 26, blackcurrant, blueberry, cranberry, raspberry, and strawberry have had their lignan content presented on a fw basis (Milder et al., 2005; Schwartz and Sontag, 2006; Thompson et al., 2006; Penalvo et al., 2008; Clarke et al., 2013a). The levels of matairesinol were very low and similar in all berries (n.d.-0.007 mg/kg fw). Fairly low levels of secoisolariciresinol (n.d.$0.256 \mathrm{mg} / \mathrm{kg} \mathrm{fw}$ ), lariciresinol (0.009-0.082 mg/kg fw), and pinoresinol (0.001-0.177 $\mathrm{mg} / \mathrm{kg} \mathrm{fw}$ ) were reported for all berries except strawberries. Strawberries consistently contained higher levels of these lignans: secoisolariciresinol $0.05-1.33 \mathrm{mg} / \mathrm{kg} \mathrm{fw}$, lariciresinol $0.229-1.17 \mathrm{mg} / \mathrm{kg} \mathrm{fw}$, and pinoresinol $0.208-2.12 \mathrm{mg} / \mathrm{kg} \mathrm{fw}$.

\subsubsection{Lignans in legumes}

Lignans are also found in legumes. The levels of secoisolariciresinol, lariciresinol, matairesinol, and pinoresinol in these foodstuffs are presented in Table 27. There is a wide range in lignan content both between different legume species and between varieties of the same species. In addition to the lignans shown in Table 27, syringaresinol and medioresinol were quantified in peanut, soybean, mung bean sprout, kidney bean, and snap pea. The levels of syringaresinol in these legumes were $0.81 \mathrm{mg} / \mathrm{kg} \mathrm{dw}$ in peanut, $0.5-3.06 \mathrm{mg} / \mathrm{kg} \mathrm{fw}$ in soybean, not detectable in mung bean 
sprout, $0.08 \mathrm{mg} / \mathrm{kg} \mathrm{fw}$ in kidney bean, and $0.08 \mathrm{mg} / \mathrm{kg} \mathrm{fw}$ in snap pea (Smeds et al., 2007; Penalvo et al., 2008). Whereas no medioresinol was detected in peanut, mung bean, kidney bean, and soybean contained $0.13-0.23 \mathrm{mg} / \mathrm{kg} \mathrm{fw}$ and snap pea $0.05 \mathrm{mg} / \mathrm{kg}$ fw (Smeds et al., 2007; Penalvo et al., 2008). Additionally, peanuts contained $0.20 \mathrm{mg}$ 7-hydroxymatairesinol/kg dw (Smeds et al., 2007).

Table 27: Lignan content ( $\mathrm{mg} / \mathrm{kg}$ fresh weight) in legumes

\begin{tabular}{|c|c|c|c|c|}
\hline Legume & Secoisolariciresinol & Lariciresinol & Matairesinol & Pinoresinol \\
\hline Peanut (Arachis hypogaea) & $0.253-0.97$ & $0.009-0.41$ & $0.001-0.05$ & n.d.-o.0o8 \\
\hline Peanut (Arachis hypogaea) ${ }^{1}$ & $1.16-2.98$ & 0.98 & n.q. -0.19 & 0.32 \\
\hline Pigeon pea (Cajanus cajan) ${ }^{1}$ & $0.19-0.5$ & & n.d. & \\
\hline Chickpea (Cicer arietinum) & $0.006-0.3652$ & 0.018 & $0.001-0.0294$ & 0.05 \\
\hline Chickpea (Cicer arietinum) ${ }^{1}$ & $0.07-0.08$ & & n.d. & \\
\hline Soybean (Glycine max) & $0.52-0.8$ & $0.69-0.996$ & n.d. -0.018 & $0.33-0.89$ \\
\hline Soybean (Glycine max) ${ }^{1}$ & $0.13-2.73$ & & n.q. & \\
\hline Soy bean (Glycine max) seed & 1.404 & & n.d. & \\
\hline Soy bean (Glycine max) sprout & 0.009 & 0.007 & n.q. & 0.005 \\
\hline Lentil (Lens culinaris) & $0.012-0.1539$ & 0.163 & $0.002-0.0223$ & 0.09 \\
\hline Lentil (Lens culinaris) ${ }^{1}$ & $0-0.07$ & & n.q. & \\
\hline Lupin (Lupinus mutabilis) ${ }^{1}$ & $0.031^{2}$ & & n.d. & \\
\hline Alfalfa sprout (Medicago sativa) & n.d. -0.022 & 0,241 & n.d.- -0.001 & 0.184 \\
\hline Lima beans (Phaseoulus lunatus) & 0.101 & 0.039 & 0.001 & 0.005 \\
\hline 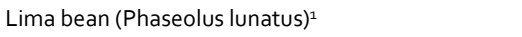 & $1.58-1.85^{2}$ & & n.q. & \\
\hline Black lentil (Phaseolus mungo) ${ }^{1}$ & $0.46-2.4$ & & $0.71-2.62$ & \\
\hline Mung bean (Phaseolus radiatus) & $0.299-3.18$ & $0.125^{-2.12}$ & 0.004 & 0.009 \\
\hline Mung bean (Phaseolus radiatus) ${ }^{1}$ & $1.72^{2}$ & & n.d. & \\
\hline Mung bean (Phaseolus radiatus) sprouts & n.d. -0.97 & $0.185-0.32$ & $0.001-0.01$ & $0.131-0.33$ \\
\hline Common bean (Phaseouls vulgaris), various types & $0.032-0.66$ & $0.018-1.28$ & n.d. -0.027 & $0.002-0.031$ \\
\hline Common bean (Phaseouls vulgaris), ${ }^{1}$ various types & $0.56-1.53$ & & n.d. -1.2 & \\
\hline Common bean (Phaseolus vulgaris), haricots verts & $0.287-0.663$ & 0.328 & $0.006-0.025$ & 0.026 \\
\hline Common bean (Phaseolus vulgaris), ${ }^{1}$ haricots verts & 10.09 & & n.q. & \\
\hline Pea (Pisum sativum) ${ }^{1}$ & $0.03-0.13$ & & $0-0.01$ & \\
\hline Snap pea (Pisum sativum var. macrocarpon) & n.d. & 0.62 & n.d. & 0.97 \\
\hline Broad bean (Vicia faba) & 0.012 & 0.062 & 0.001 & 0.042 \\
\hline Broad bean $\left(\right.$ Vicia faba) ${ }^{1}$ & $0.26-1.02$ & & n.q. -1.31 & \\
\hline Cowpea, Black-eyed (Vignia unguiculata) ${ }^{1}$ & $1.95-1.96$ & & n.d. & \\
\hline
\end{tabular}

Note: ${ }^{1} \mathrm{mg} / \mathrm{kg}$ dry weight

${ }^{2}$ secoisolariciresinol frequently reported as sum of anhydrosecoisolariciresinol and secoisolariciresinol (Mazur et al., 1998b).

n.d. = non-detectable; n.q. = non-quantifiable.

Source: Mazur \& Adlercreutz, 1998; Mazur et al., 1998b; Horn-Ross et al., 2000; Liggins et al., 2000c; Milder et al., 2005; Schwartz and Sontag, 2006; Thompson et al., 2006; Kuhnle et al., 2007, 2008b; Penalvo et al., 2008; Clarke et al., 2013a; Konar, 2013.

Flour made of lentil, soy and chickpea have been analysed for their lignan content. Green lentil flour and red lentil flour contained similar levels of secoisolariciresinol ( 1.74 and $0.82 \mathrm{mg} / \mathrm{kg} \mathrm{dw}$ ), lariciresinol ( 1.64 and $1.77 \mathrm{mg} / \mathrm{kg} \mathrm{dw}$ ), matairesinol ( 0.49 and $0.47 \mathrm{mg} / \mathrm{kg} \mathrm{dw}$ ), and pinoresinol (1.74 and $1.20 \mathrm{mg} / \mathrm{kg} \mathrm{dw}$ ) (Durazzo et al., 2013). Soy flour contained $1.30 \mathrm{mg}$ secoisolariciresinol $/ \mathrm{kg} \mathrm{dw}$ and trace levels of matairesinol (Liggins et al., 2000c; Mazur et al., 1996). Chickpea flour contained $0.222 \mathrm{mg}$ lariciresinol $/ \mathrm{kg} \mathrm{dw}$ and $0.085 \mathrm{mg}$ pinoresinol $/ \mathrm{kg} \mathrm{dw}$ (Durazzo et al., 2013). No secoisolariciresinol or matairesinol was detected. 


\subsubsection{Lignans in vegetables}

Investigators have reported their findings of lignan content in vegetables either on a dw or a fw basis, but because of lack of information, it was not possible to recalculate all of the data to $\mathrm{fw}$. The levels of lignans in vegetables are therefore presented in two tables; Table 28 (on a fw basis) and Table 29 (on a dw basis). Vegetables contain many lignans but generally in low amounts as evident from the data in the Tables. Some vegetables contain high amounts of individual lignans or a few lignans. The vegetable(s) reported to contain the highest amount of secoisolariciresinol was asparagus (0.68-7.43 mg/kg fw) and pumpkin (38.7 mg/kg dw), lariciresinol was highest in broccoli (0.82-9.72 mg/kg fw), matairesinol in Japanese horseradish $(0.71 \mathrm{mg} / \mathrm{kg} \mathrm{fw}$ ) and sweet potato $(4.63 \mathrm{mg} / \mathrm{kg} \mathrm{dw})$, syringaresinol in avocado ( $4.47 \mathrm{mg} / \mathrm{kg} \mathrm{fw})$, pinoresinol in curly kale (16.91 mg/kg fw), and medioresinol in avocado $(2.41 \mathrm{mg} / \mathrm{kg} \mathrm{fw})$.

\subsubsection{Lignans in nuts}

The lignan content has been determined in a double handful of nut-producing species (Table 30). In total, six lignans have been detected in consumed nuts: secoisolariciresinol, lariciresinol, isolariciresinol, matairesinol, syringaresinol, and pinoresinol. As indicated by the footnote in Table 30, some values are reported on a $\mathrm{dw}$ basis instead of a fw basis. Because the moisture content of harvested nuts typically is $<10 \%$ (but fluctuates significantly depending on the time of harvest and the climate conditions during growth and storage) (Alasalvar \& Shahidi, 2009), a rough conversion of the $d w$ data to quantity in fw could be obtained by dividing the $d w$ quantity with 1.1.

Levels of lignans differ between types of nut. The highest lignan levels have been reported in Brazil nuts and cashew nuts. Almonds, cashew nuts, and walnuts were also analysed for their 7 -hydroxymatairesinol content. It was found that cashew nuts contain $0.022 \mathrm{mg} / \mathrm{kg} \mathrm{dw}$ and walnuts $0.032 \mathrm{mg} / \mathrm{kg} \mathrm{dw}$, whereas no 7-hydroxymatairesinol was found in almonds (Smeds et al., 2007). In a flour made of sweet chestnut the levels of secoisolariciresinol, lariciresinol, pinoresinol, and isolariciresinol were 6.04, $0.899,0.724$ and $2.14 \mathrm{mg} / \mathrm{kg} \mathrm{dw}$, respectively (Durazzo et al., 2013). 
Table 28: Lignan content ( $\mathrm{mg} / \mathrm{kg}$ fresh weight) in vegetables

\begin{tabular}{|c|c|c|c|c|c|c|}
\hline Vegetable & $\begin{array}{l}\text { Secoisilarici- } \\
\text { resinol }\end{array}$ & Lariciresinol & Matairesinol & $\begin{array}{l}\text { Syringa- } \\
\text { resinol }\end{array}$ & Pinoresinol & $\begin{array}{l}\text { Medio- } \\
\text { resinol }\end{array}$ \\
\hline Asparagus & $0.684-7.43$ & $0.47-1.78$ & $0.02-0.14$ & 0.58 & $0.49-1.22$ & $0.03-0.05$ \\
\hline Avocado & 0.47 & 0.31 & 0.06 & 4.47 & 2.72 & 2.41 \\
\hline Broccoli & $0.058-0.44$ & $0.82-9.72$ & n.d. -0.001 & & $0.061-3.15$ & \\
\hline Brussel sprout & 0.34 & 4.93 & n.d. & & 2.2 & \\
\hline Cabbage & 0,026 & 0,323 & 0,001 & & 0,442 & \\
\hline Carrot & $0.041-1.77$ & $0.021-0.6$ & n.d. & & $0.001-0.19$ & \\
\hline Celeriac & $0.40-1,28$ & $0.25-0.77$ & & & & \\
\hline Cherry tomatoes & $0.16-0.17$ & $0.3^{8-0.43}$ & n.d. & $0.03-0.06$ & $0.11-0.19$ & 0.03 \\
\hline Chinese cabbage & 0.25 & 0.77 & n.d. & 0.04 & 0.17 & 0.02 \\
\hline Collards & 0.059 & 0.667 & 0.004 & & 0.248 & \\
\hline Cucumber & $0.02-0.41$ & $0.01-0.65$ & n.d. & n.d. & n.d. $-0,01$ & n.d. \\
\hline Curly kale & 0.19 & 5.99 & 0.12 & & 16.91 & \\
\hline Dried gourd & 1.08 & 0.78 & n.d. & n.d. & n.d. & n.d. \\
\hline Endive & 0.14 & 0.15 & n.d. & & 0.09 & \\
\hline Edible burdock (Arctium lappa) & 2.51 & 0.95 & 0.39 & 0.09 & 0.74 & 0.1 \\
\hline French bean & 0.29 & 2.2 & n.d. & & 0.24 & \\
\hline Garlic & $0.27-0.55$ & $0.54-2.86$ & n.d.-0.37 & & $0.45-4.8$ & n.d. \\
\hline Ginger & n.d. & n.d. & 0.16 & 0.05 & 0.15 & n.d. \\
\hline Lettuce & $0.049-0.08$ & $0.024-0.05$ & n.d.-0.01 & & $0.006-0.04$ & \\
\hline Okra & 2.21 & 0.1 & n.d. & 0,11 & 0.03 & \\
\hline Onion & $0.18-0.22$ & $0.003-0.19$ & n.d. $-0,09$ & & n.d. -0.008 & \\
\hline Parsley & 0.7 & & n.d. & & & \\
\hline Perilla & $0.64-1.38$ & $1.02-1.23$ & n.d. & $0.24-1.02$ & $1.18-1.74$ & $0.31-0.46$ \\
\hline Japanese pumpkin & 0.09 & 0.14 & n.d. & n.d. & 0.01 & n.d. \\
\hline Pumpkin & $0.009-0.2$ & $0.037-0.29$ & n.d. -0.002 & n.d. & n.d. -0.002 & n.d. \\
\hline Pumpkin seed & n.d. -5.1 & & n.d.-0.11 & & & \\
\hline Sauerkraut & 0.67 & 1.16 & n.d. & & 1.33 & \\
\hline Spinach & $0.013-0.08$ & $0.105^{-1.1}$ & n.d. -0.001 & n.d. & $0.019-0.31$ & n.d. \\
\hline Japanese mustard spinach & 1.78 & 1.89 & n.d. & 0.32 & 1.36 & 0.19 \\
\hline Sweet pepper & $0.05-0.09$ & $0.73-1.64$ & n.d. & n.d. -0.04 & $0.01-0.06$ & n.d.-0.01 \\
\hline Sweet potatoes & n.d. -0.65 & $0.14-0.79$ & $0.02-0.41$ & n.d. & $0.011-0.16$ & n.d. \\
\hline White potato & 0.004 & 0.006 & n.q. & & 0.002 & \\
\hline Turnip rape & 0.38 & 2.23 & n.d. & 0.18 & 2.02 & 0.29 \\
\hline Tomato & $0.01-0.03$ & $0.06-0.42$ & n.d. & $0.02-0.07$ & $0.05-0.14$ & $0.02-0.05$ \\
\hline Wasabi (Japanese horseradish) & 0.92 & 6.59 & 0.71 & 0.28 & 0.91 & 0.12 \\
\hline White cabbage & 0.08 & 2.12 & n.d. & & 5.68 & \\
\hline Winter squash & 0.134 & 0.911 & 0.001 & & 0.088 & \\
\hline Zucchini & $0.018-0.36$ & $0.027-0.64$ & n.d. -0.001 & & $0.005-0.37$ & \\
\hline
\end{tabular}

Note: $\quad$ n.d. = non-detectable; n.q. = non-quantifiable.

Source: Horn-Ross et al., 2000; Milder et al., 2005; Schwartz \& Sontag, 2006; Thompson et al., 2006; Kuhnle et al., 2008b; Penalvo et al., 2005, 2008; Clarke et al., 2013 a. 
Table 29: Lignan content ( $\mathrm{mg} / \mathrm{kg}$ dry weight) in vegetables

\begin{tabular}{|c|c|c|c|c|c|c|}
\hline Vegetable & $\begin{array}{l}\text { Secoisolarici- } \\
\text { resinol }\end{array}$ & $\begin{array}{l}\text { Larici- } \\
\text { resinol }\end{array}$ & $\begin{array}{l}\text { Matai- } \\
\text { resinol }\end{array}$ & $\begin{array}{l}\text { Syringa- } \\
\text { resinol }\end{array}$ & $\begin{array}{l}\text { Pino- } \\
\text { resinol }\end{array}$ & $\begin{array}{l}\text { Medio- } \\
\text { resinol }\end{array}$ \\
\hline Asparagus & & & $1-1.4$ & & & \\
\hline Aubergine $^{1,2}$ & $0.71-1.3$ & $6.7-9.7$ & n.d. & $0.29-3$ & $4-8.5$ & $0.57-2.5$ \\
\hline Beetroot & 1 & & n.q. & & & \\
\hline Broccoli & $4.14-5.99$ & & $0.02-0.23$ & & & \\
\hline Cabbage & 0,33 & & n.q. & & & \\
\hline Cabbage, Green & 0.98 & & 0.04 & & & \\
\hline Cabbage, Red 3 & $0.54-1.41$ & 19.8 & n.d.-0.04 & & 10 & \\
\hline Cabbage, Savoy & $1.90-3.86$ & & $0.01-0.0481$ & & & \\
\hline Carrot & $3.7-6.77$ & & n.q. -1.49 & & & \\
\hline Cauliflower 3,8 & $0.5^{-1.61}$ & $4.22-15.5$ & n.d. -0.03 & 2.9 & $7.25-9.44$ & 2.44 \\
\hline Celeriac & 1.74 & & 0.03 & & & \\
\hline Celery 4 & $1.1-1.2$ & 1.45 & n.d.-n.q. & 0.91 & 3.91 & 0.72 \\
\hline Cherry & 0.11 & & 0.03 & & & \\
\hline Chicory 5 & $3.35-3.4$ & 1.2 & n.d.-0.14 & & 5 & \\
\hline Garlic & 3.8 & & n.q. & & & \\
\hline Leek ${ }^{4}$ & $3.45-7.02$ & 3.36 & n.d. -0.03 & & 0.27 & \\
\hline Lettuce, Iceberg 5 & $0.763-1.8$ & 0.4 & n.d. -0.135 & & n.d. & \\
\hline Lettuce, Round & 1.51 & & 0.04 & & & \\
\hline Marrow & 1.99 & & n.q. & & & \\
\hline Onion & $0.83-2.8$ & & $0.02-0.08$ & & & \\
\hline Spring onion & 6.71 & & 0.06 & & & \\
\hline Parsnip & 1.81 & & 0.99 & & & \\
\hline Pea & $0.008-0.032$ & & $0.009-0.035$ & & & \\
\hline Potato & 0.1 & & 0.06 & & & \\
\hline Red potato & $0.036-0.085$ & & $0.009-0.514$ & & & \\
\hline Pumpkin & 38.7 & & 0.04 & & & \\
\hline Pumpkin seed & n.d. & 0.749 & n.d. & n.d. & 0.101 & 1.92 \\
\hline Radish $^{2,6}$ & n.d. -0.62 & $3 \cdot 5-4.8$ & n.d. -0.25 & $0.5-5.3$ & $0.5-7.2$ & $0.25-2.0$ \\
\hline Rhubarb & 0.01 & & 0.02 & & & \\
\hline Spinach & 0.5 & & 0.01 & & & \\
\hline Swede & $0.38-0.53$ & & n.q. -0.02 & & & \\
\hline Sweet pepper & $0.78-1.2$ & & n.d.-0.07 & & & \\
\hline Sweet potato & 5.17 & & 4.63 & & & \\
\hline Tomato & 0.61 & & 0.02 & & & \\
\hline Turnip5 & $1.31-1.8$ & 20.2 & n.d.-0.02 & 6.6 & 0.8 & 0.8 \\
\hline Watercress 7 & $6.25-7.5$ & 10.2 & n.d. -0.03 & 0.3 & 2.1 & 0.3 \\
\hline Witloof & 1.75 & & 0.15 & & & \\
\hline Zucchini & $5.53-8.17$ & & n.d.-n.q. & & & \\
\hline
\end{tabular}

Note: ${ }^{1}$ recalculated, assuming moisture content of $93 \%$

${ }^{2}$ recalculated, assuming moisture content of $94 \%$

3 recalculated, assuming moisture content of $91 \%$

4 recalculated, assuming moisture content of $89 \%$

5 recalculated, assuming moisture content of $95 \%$

${ }^{6}$ recalculated, assuming moisture content of $96 \%$

7 recalculated, assuming moisture content of $90 \%$

${ }^{8}$ recalculated, assuming moisture content of $92 \%$

n.d. $=$ non-detectable $;$ n.q. $=$ non-quantifiable

Source: Mazur \& Adlercreutz, 1998; Liggins et al., 2000c; Milder et al., 2005; Kuhnle et al., 2007, 2009b; Penalvo et al., 2005, 2008; Smeds et al., 2012. 
Table 30: Lignan content ( $\mathrm{mg} / \mathrm{kg}$ fresh weight if not indicated otherwise) in nuts

\begin{tabular}{|c|c|c|c|c|c|}
\hline Nut & $\begin{array}{l}\text { Secoisolarici- } \\
\text { resinol }\end{array}$ & Larici-resinol & Matai-resinol & $\begin{array}{l}\text { Syringa- } \\
\text { resinol }\end{array}$ & Pino-resinol \\
\hline Almonds & $0.70-0.84$ & 0.322 & n.d. -0.003 & & 0.09 \\
\hline Almonds ${ }^{1}$ & $1.07-1.59$ & 2.33 & n.q. -0.24 & 0.40 & 2.08 \\
\hline Brazil nuts & 7.7 & & 0.12 & & \\
\hline Cashew nuts & $0.375^{-1.66}$ & $0.605-4.96$ & $0.003 .-0.05$ & & n.d.-0.011 \\
\hline Cashew nuts ${ }^{1}$ & $2.57-3.16$ & 3.07 & $0.04-0.55$ & 0.26 & 0.19 \\
\hline Chestnuts (Castanea sativa) & 1.727 & 0.078 & 0.005 & & 0.056 \\
\hline Coconut & 0.3 & & 0.02 & & \\
\hline Hazelnut & $0.53-0.605$ & 0.143 & $0.04-0.012$ & & 0.011 \\
\hline Hazelnut $^{1}$ & 1.19 & & 0.04 & & \\
\hline Pecans & $0.13-0.148$ & 0.084 & $0.006-0.04$ & & 0.012 \\
\hline Pine nuts & 0.46 & & 0.25 & & \\
\hline Pistachios & 0.446 & 1.23 & 0.001 & & 0.312 \\
\hline Pistachios $^{1}$ & 0.96 & & n.d. & & \\
\hline Walnuts $^{1}$ & $0.99-1.63$ & 0.47 & $0.05-0.6$ & 0.24 & 0.34 \\
\hline Walnuts & $0.78-1.4$ & 0.072 & $0.002-0.05$ & & 0.002 \\
\hline
\end{tabular}

Note: $\quad{ }^{1} \mathrm{mg} / \mathrm{kg}$ dry weight.

n.d. = non-detectable; n.q. = non-quantifiable

Source: Mazur \& Adlercreutz, 1998; Milder et al., 2005; Schwartz and Sontag, 2006; Thompson et al., 2006; Smeds et al., 2007; Kuhnle et al., 2008b.

\subsubsection{Lignans in oil seeds}

Oil seeds may be very rich in lignans. Particularly high lignan levels have been found in sesame seeds (Table 31). The levels of pinoresinol is about $300-470 \mathrm{mg} / \mathrm{kg} \mathrm{dw}$ and the level of lariciresinol between 130 and $160 \mathrm{mg} / \mathrm{kg} \mathrm{dw}$. Intermediate levels of lignans were found in hemp and camelina seeds, with hemp seeds being particularly rich in meioresinol (40 mg/kg dw). In addition to the lignans listed in Table 31, some seeds were analysed for 7-hydroxymataresinol. Camelina seeds contained $0.38 \mathrm{mg} / \mathrm{kg} \mathrm{dw}$ and sesame seeds $72.09-174.7 \mathrm{mg} / \mathrm{kg} \mathrm{dw}$, whereas no 7-hydroxymataresinol was detected in hempseeds (Smeds et al., 2012).

\subsubsection{Lignans in cereals}

Many kinds of cereals have been analysed for their lignan content. The results from most of these studies are presented in Table 32. Flaxseed has the highest reported levels of secoisolariciresinol, lariciresinol, pinoresinol, and matairesinol, as well as one of the highest reported levels of secoisolariciresinol diglucoside $(14,100-30,000 \mathrm{mg} / \mathrm{kg}$ $\mathrm{dw}$ ) in any foodstuff. The levels of secoisolariciresinol diglucoside given for flaxseed is the sum of (+)- and (-)-secoisolariciresinol diglucoside. The content of secoisolariciresinol diglucoside was examined in a few other cereal products. In these studies, it was shown that flaxseed press cake contains $12,700-16,300 \mathrm{mg} / \mathrm{kg} \mathrm{dw}$, defatted flaxseed flour $11,700-24,100 \mathrm{mg} / \mathrm{kg} \mathrm{dw}$, whole seed flaxseed flour 7,300$13,300 \mathrm{mg} / \mathrm{kg} \mathrm{dw}$, and commercially available flaxseed hulls 16,400-33,900 mg/kg dw (Johnsson et al., 2000; Eliasson et al., 2003; Beejmohun et al., 2007; Hao \& Beta 2012). It is not surprising that the levels of secoisolariciresinol diglucoside are high in flaxseed 
hulls because lignans are predominantly present in the outer layers of grains and cereals (Dixon, 2004).

The highest levels of syringaresinol were reported in rye. In addition to the lignans reported in Table 32, isolariciresinol levels were determined in rye bran, wheat bran, soft wheat seeds, and soft wheat seed flour. The levels were non-detectable in soft wheat seeds and soft wheat seed flour, and were $2.95 \mathrm{mg} / \mathrm{kg} \mathrm{dw}$ in rye bran and 2.97 $\mathrm{mg} / \mathrm{kg} \mathrm{dw}$ in wheat bran.

Greater insight into how the level of lignans in flaxseed is affected by growing location, harvest year, seeding time, and variety was supplied in the study of Thompson et al. (1997). These investigators studied three flaxseed varieties (Linott, McGregor and NorLin) grown in four locations during five seasons. The seeds were sown in early May three seasons and in early May or June the last season. Lignan levels differed between varieties and within variety because of the environment (site of cultivation). The Linott variety was the only flaxseed for which the harvest year was significant. Sowing time did not influence the lignan content (Thompson et al., 1997).

Table 31: Lignan content ( $\mathrm{mg} / \mathrm{kg}$ dry weight) in oil seeds

\begin{tabular}{|c|c|c|c|c|c|c|}
\hline Seed & $\begin{array}{l}\text { Secoisolarici- } \\
\text { resinol }\end{array}$ & Larici-resinol & Matairesinol & $\begin{array}{l}\text { Syringa- } \\
\text { resinol }\end{array}$ & Pino-resinol & $\begin{array}{l}\text { Medio- } \\
\text { resinol }\end{array}$ \\
\hline Camelina seeds & 15.17 & 21.79 & 0.33 & 7.12 & 0.983 & 6.56 \\
\hline Hemp seeds & 0.755 & 0.574 & 0.047 & 0.27931 & 3.1 & 40.08 \\
\hline Poppy seed ${ }^{1}$ & n.d. -0.14 & 0.11 & n.d. -0.12 & & n.d. & \\
\hline Sesame seeds & $0.9-6.73$ & $130.60-159.44$ & $6.08-11.61$ & $2.05-5.56$ & $303.97-471.36$ & $41.53-94.76$ \\
\hline Sesame seed ${ }^{2}$ & n.d. -0.66 & $10.52-94.7$ & $1.23-4.81$ & & $68.15^{-293.31}$ & \\
\hline Sunflower seed ${ }^{2}$ & $0.262-1.278$ & $1.50-6.71$ & n.d.- -0.03 & & $0.339-1.67$ & \\
\hline Sunflower seed & 6.1 & & n.d. & & & \\
\hline
\end{tabular}

Note: ${ }^{1}$ recalculated, assuming moisture content of $8 \%$

${ }^{2} \mathrm{mg} / \mathrm{kg}$ fresh weight.

n.d. = non-detectable; n.q. = non-quantifiable.

Source: Mazur \& Adlercreutz, 1998; Horn-Ross et al., 2000; Milder et al., 2005; Schwartz and Sontag, 2006; Thompson et al., 2006; Smeds et al., 2007, 2012; Kuhnle et al., 2008b.

Smeds et al. (2009) investigated the lignan content in winter rye, spring wheat, and spring oat grown at different locations and during different seasons. They observed a large variation in lignan content. It was suggested that this variation could be due to the different years and locations where the cereals were cultivated. Another factor contributing to the variability could be the different genetic backgrounds of the various cereal cultivars analysed. For rye, it was found that the grain size was inversely associated with the lignan content, i.e. smaller grains contained higher levels of lignans.

\subsubsection{Lignans in other foodstuffs}

This category encompasses a few food items that are not easily placed in the other groups, but might be important for the exposure of some consumers to phytoestrogens via food and feed. The food items in Table 33 generally contain low 
levels of lignans, with black licorice, okra, and caraway seeds being a few exceptions in their content of secoisolariciresinol.

In addition to the lignans in Table 33, medioresinol was analysed in a few food items. Whereas medioresinol could not be detected in American "groundnut", okra, and Japanese yam, a level of $0.26 \mathrm{mg} / \mathrm{kg}$ fw was found in cocoa and $0.02 \mathrm{mg} / \mathrm{kg} \mathrm{fw}$ in East Indian lotus root.

Milder et al. (2005) compared the lignan content in cocoa with that in chocolate. Modest levels of two lignans (secoisolariciresinol and lariciresinol) were detected in cocoa. Analysis of the chocolate revealed similar levels of lariciresinol to that in cocoa (0.08 mg/kg fw) but no secoisolariciresinol was identified. Thompson et al. (2006) reported the prepared black licorice to contain comparative high amounts $(3.42 \mathrm{mg} / \mathrm{kg}$ $\mathrm{fw}$ ) of secoisolariresinol.

One food item not mentioned in Table 33 is muesli, which is a mix of various ingredients. The lignan content was investigated by Milder et al. (2005) and Kuhnle et al. (2009a). Not unexpectedly, the studies indicate that the levels vary greatly between brands depending on what ingredients have been used.

The ripe dried pod of the carob tree (St. John's bread) is ground to carob powder and used in, for example, health foods. Analysis of carob flour (Ceratonia siliqua) identified 2.72-2.95 mg secoisolariciresinol/kg dw, 2.54-2.77 mg lariciresinol $/ \mathrm{kg} \mathrm{dw}, 2.72-2.95 \mathrm{mg}$ pinoresinol $/ \mathrm{kg} \mathrm{dw}$, and $4.43-6.38 \mathrm{mg}$ isolariciresinol $/ \mathrm{kg} \mathrm{dw}$ (Durazzo et al., 2014). 
Table 32: Lignan content (mg/kg dry weight) in cereals, cereal flours, and cereal meals

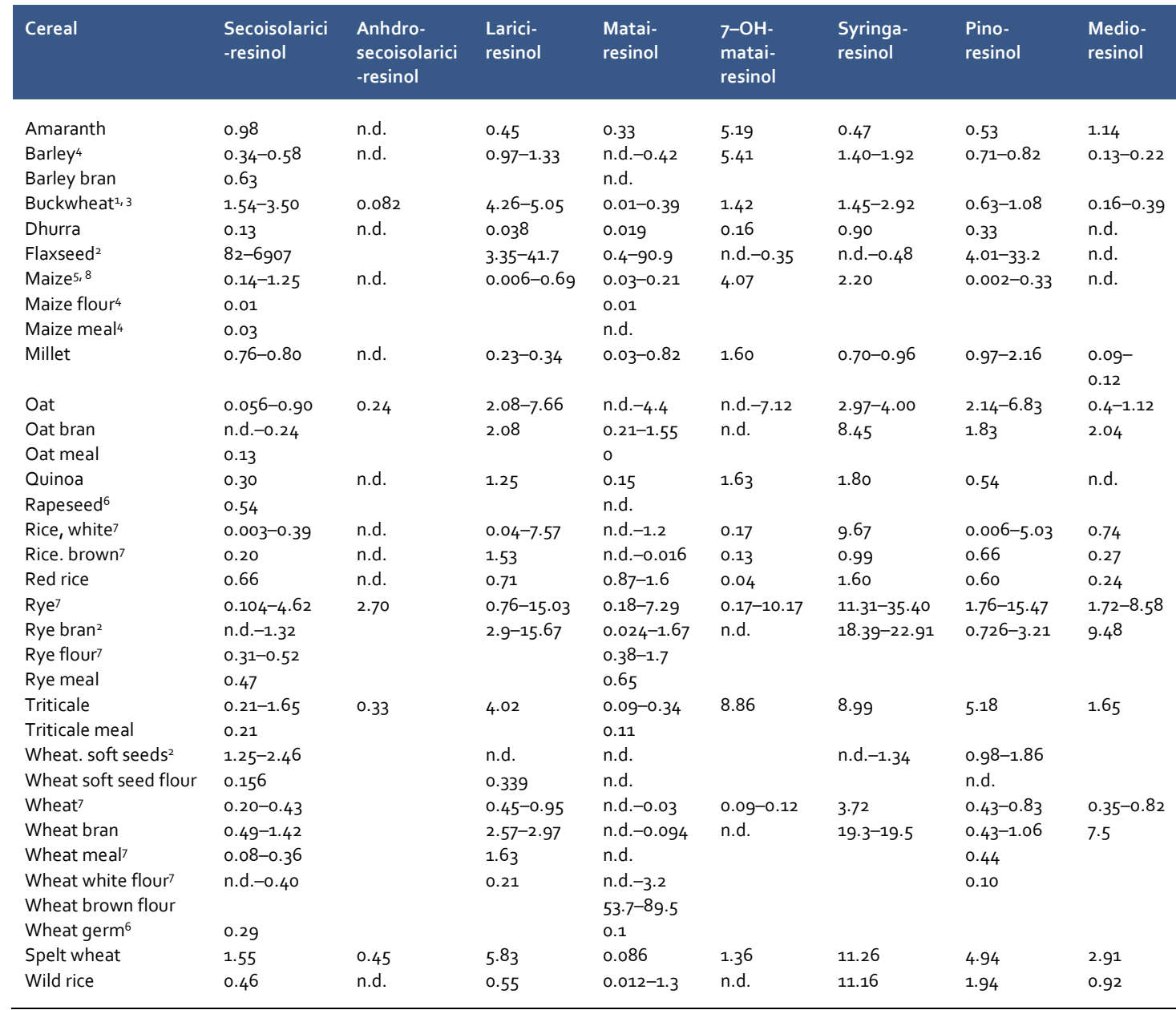

Note: ${ }^{1} \mathrm{mg} / \mathrm{kg}$ fresh weight

${ }^{2}$ secoisolariciresinol reported as sum of anhydrosecoisolariciresinol and secoisolariciresinol (Mazur et al., 1996;

Dinelli et al., 2007)

3 recalculated, assuming moisture content of $15 \%$

4 recalculated, assuming moisture content of $12 \%$

5 recalculated, assuming moisture content of $21 \%$

${ }^{6}$ recalculated, assuming moisture content of $9 \%$

7 recalculated, assuming moisture content of $14 \%$

8 recalculated, assuming moisture content of $76 \%$.

Source: Mazur et al., 1996; Nilsson et al., 1997; Mazur \& Adlercreutz, 1998; Setchell et al., 1999; Liggins et al., 2000c; Kraushofer \& Sontag, 2002; Eliasson et al., 2003; Begum et al., 2004; Milder et al., 2005; Penalvo et al., 2005; Schwartz and Sontag, 2006; Thompson et al., 2006; Dinelli et al., 2007; Smeds et al., 2007, 2009, 2012; Kuhnle et al., 2008b, 2009a; Durazzo et al., 2013. 
Table 33: Lignan content ( $\mathrm{mg} / \mathrm{kg}$ fresh weight) of some spices and less common food items or herbs on the Nordic market

\begin{tabular}{|c|c|c|c|c|c|}
\hline Product & $\begin{array}{l}\text { Secoisolarici- } \\
\text { resinol }\end{array}$ & $\begin{array}{l}\text { Larici- } \\
\text { resinol }\end{array}$ & $\begin{array}{l}\text { Matai- } \\
\text { resinol }\end{array}$ & $\begin{array}{l}\text { Pino- } \\
\text { resinol }\end{array}$ & $\begin{array}{l}\text { Syringa- } \\
\text { resinol }\end{array}$ \\
\hline Arrowhead (Sagittaria trifolia) & n.d. & 0.03 & n.d. & 0.04 & 0.2 \\
\hline Arrow root & 0.01 & & 0.01 & & \\
\hline Bamboo & 0.38 & n.d. & n.d. & n.d. & 0.03 \\
\hline Black licorice & n.d-3.415 & 0.396 & n.d. -0.013 & 0.327 & \\
\hline Caraway seed ${ }^{1}$ & 2.21 & & 0.06 & & \\
\hline Cocoa & 0.08 & 0.26 & & & n.d. \\
\hline East indian lotus root & 0.01 & 0.31 & n.d. & 0.27 & n.d. \\
\hline Fenugreek (Trigonella foenumgraecum) ${ }^{1}$ & 0.086 & & n.q. & & \\
\hline Kudzu root (Pueraria sp.) $)^{1}$ & 0.31 & & n.q. & & \\
\hline Japanese yam (Dioscorea opposita) & 0.13 & 0.1 & 0.01 & n.d. & n.d. \\
\hline Peanut (Apios americana) $)^{1}$ & $0.21-0.58$ & & $0.02-0.05$ & & \\
\hline Clover seed $^{1}$ & 0.13 & & 0.04 & & \\
\hline Clover sprouts & n.d. & & n.d. & & \\
\hline Red clover $^{1}$ & n.q. ${ }^{2}$ & & n.q. & & \\
\hline
\end{tabular}

Note: $\quad{ }^{1} \mathrm{mg} / \mathrm{kg}$ dry weight.

${ }^{2}$ secoisolariciresinol reported as sum of anhydrosecoisolariciresinol and secoisolariciresinol (Mazur et al., 1998b).

n.d. = non-detectable; n.q. = non-quantifiable.

Source: Mazur \& Adlercreutz, 1998; Horn-Ross et al., 2000; Milder et al., 2005; Schwartz \& Sontag, 2006; Thompson et al., 2006; Smeds et al., 2007, 2012; Kuhnle et al., 2008b.

\subsubsection{Lignans in dietary supplements}

Penalvo et al. (2004) studied the phytoestrogen content of dietary soy-based supplements. More specifically, the authors analysed seven isoflavone concentrates in the form of tablets or capsules from Finland, Israel, and the USA, and seven soy protein-based products in the same form from Finland, France, Netherlands, Norway, and the USA. Although most of these products intended to supply the consumer with soy isoflavones, it was noted that they also contained lignins but the levels varied considerably between preparations. All fourteen products contained secoisolariciresinol (0.06-74.2 mg/kg), isolariciresinol (0.22-68.8 mg/kg), and syringaresinol (0.19-78.3 mg/kg). Thirteen products contained lariciresinol (2.41-41.7 $\mathrm{mg} / \mathrm{kg}$ ), twelve products pinoresinol $(0.10-92.1 \mathrm{mg} / \mathrm{kg}$ ) and ten products matairesinol (0.01-0.46 mg/kg). Therefore, when performing an exposure assessment of phytoestrogens from isoflavone food supplements, the contribution from lignans needs to be considered.

\subsubsection{Influence of storage and processing on lignant content}

A few investigators have compared the lignan content of cooked and raw vegetables. Milder et al. (2005) reported an approximately 30\% loss in lignan content after boiling and frying of vegetables. Penalvo et al. (2008) also reported losses after boiling of 
asparagus, mung bean sprouts, and soybean sprouts. However, the levels of lignans were increased in soybean sprouts. Increases could be due to release from the food matrix or degradations from one lignan into another. Kuhnle and colleagues (2007) reported only small decreases of lignan levels in vegetables after cooking. When lignan levels were compared in boiled and raw, white and brown rice, the processing was shown to result in small decreases in the lignan content (Liggins et al., 2000c; Milder et al. 2005; Kuhnle et al. 2009a).

Some studies have compared the lignan content of raw and processed legumes. Cooking did not significantly alter the level of lignans (Kuhnle et al., 2007; Penalvo et al., 2008). Kuhnle and co-workers (2008b) analysed the lignan content in roasted peanuts and found that there were higher levels of matairesinol and secoisolariciresinol than in the unroasted peanuts. Kuhnle et al. (2008b) measured the levels of secoisolariciresinol and matairesinol in roasted and salted pistachios. The levels found were not substantially different from those found in unroasted pistachios. However, sun-drying harvested pistachio nuts for three days reduced the concentration of daidzein, genistein, and daidzin in the nuts by about $35-40 \%$, but not the concentration of genistein (Bolling et al., 2011). It also reduced the level of the stilbene trans-resveratrol (a reduction by more than $80 \%$ ).

Smeds et al. (2012) investigated what effect dehulling of hemp and sesame seeds would have on lignan content. The levels of the analysed lignans were much lower after dehulling. The lower levels are most likely due to lignans being present in the outer layers of grains and cereals (Dixon, 2004). Thus, depending on the phytoestrogen, processing of a plant to produce various products may influence the phytoestrogen content of the product.

Milder et al. (2005) compared the levels of secoisolariciresinol, lariciresinol, matairesinol, and pinoresinol in fresh and canned pineapple. The levels of secoisolariciresinol and pinoresinol were comparable, whereas levels of lariciresinol and matairesinol were lower in the canned product. This finding could be explained by the canning process. The little data available comparing the lignan levels in raw and cooked fruits and berries indicate very small differences and it appears that the constituents are not degraded by heat. This argument concurs to some extent with what Gerstenmeyer et al. (2013) showed in their study on thermal degradation of lignans. Heating dry foods, flax seeds, rye, and sesame seeds at $100^{\circ} \mathrm{C}$ did not degrade the tested lignans, but the lignans were found to degrade when the samples had high moisture content. Hyvärinen et al. (2006) performed an experiment to determine whether the baking process and subsequent storage (one week to two months) would affect the levels of secoisolariciresinol diglucoside in dough. It was found that secoisolariciresinol diglucoside could withstand high baking temperatures and did not degrade in storage.

Finnish investigators have performed additional studies on sourdough baking with rye (Liukkonen et al., 2003). In this cereal, lignans are mainly found in the bran layers of the grain and occur at only low levels in the flour endosperm. It was demonstrated that lignan levels remained constant both during rye germination and sourdough baking. 
The influence of thermal treatments simulating cooking processes on the content of bioactive compounds in virgin olive oil was studied by Brenes et al. (2002). Thermal oxidation of oils at $190^{\circ} \mathrm{C}$ for 25 hours still retained a high proportion of the lignans 1acetoxypinoresinol and pinoresinol, whereas other bioactives were more influenced. Lignans were most stable also after microwave heating for ten minutes, as well as after boiling an olive oil/water mixture for thirty minutes in a pressure cooker.

In conclusion, lignans are fairly stable during normal cooking and baking conditions.

\subsection{Stilbene levels in foods}

\subsubsection{Stilbene production in plants}

Stilbenes are a class of non-flavonoid 14-carbon phenolic 1,2-diarylethenes that can be found in some plants. The chemical structure of the most common stilbenes is illustrated in Figure 5. The compounds contain two phenolic rings with substituents the A ring (to the upper part in Fig. 5) usually contains two hydroxyl groups in the $m$ position, and the B ring (to the lower part in Fig. 5) may contains hydroxyl and methoxy groups in the $\mathrm{O}_{-}, \mathrm{m}-$, and $\mathrm{p}$-positions. Stilbenoids are considered phytoestrogens as they can interact with ERs, alter gene expression, and otherwise affect hormones. Of stilbenoids, the aglycone resveratrol is the most studied compound. However, it was not until 1997 that Gehm and associates demonstrated that resveratrol is an agonist for the oestrogen receptor, thereby confirming that it is a phytoestrogen.

Stilbenes are formed via the phenylpropanoid and acetate-malonate pathway. The key enzyme in the pathway is the stilbene synthase, which catalyses the formation of trans-resveratrol via condensation of 4-coumaroyl-CoA and malonyl$\mathrm{CoA}$. The central role of this enzyme was recognised when the grapevine genome was sequenced (Jaillon et al., 2007). The sequence showed that no less than 43 stilbene synthase genes were identified (20 of these were previously shown to be expressed). The stilbene synthase genes can be grouped according to their responsiveness toward external signals, including abiotic stresses or biotic signals (Jeandet et al., 2010). This organisation at the genome level is part of the background for stilbene synthesis being either constitutive or induced by biotic and abiotic elicitors.

In the plant cell, resveratrol may be methylated, oligomerised, and/or glycosylated. To enhance its stability within the cell, resveratrol is protected from oxidation and enzymatic degradation by being glycosylated (Chong et al., 2009; Krasnow and Murphy, 2004; Hall \& De Luca, 2007). An O-methyltransferase gene (romt) has been identified in grapevine, transforming resveratrol to its dimethylated derivative (Schmidlin et al., 2008) and peroxidases can oxidise resveratrol to yield the resveratrol dimer $\varepsilon$-viniferin (Ros-Barcelo et al., 2003). Although cis-isomers of stilbenes have been identified in grapevine, no stilbene isomerise has yet been detected in the plant. The site within the cell where stilbenes are synthesised and where they are stored is not very well 
understood but they seem to be linked to the lignified parts of cell walls. Under UV light, stilbenes fluoresce characteristically blue, a characteristic that is frequently used to detect them in plant tissue and chromatograms.

Stilbenes have been recognised for their antifungal properties since shortly before the Second World War (Erdtman, 1939). Langcake and co-workers noted that they not only occur constitutively in heartwood and similar plant tissues (roots, stems, canes, and grape seeds) but also in leaves following fungal infection, and implicated them as phytoalexins (Langcake \& Pryce, 1976, 1977a), i.e. they are induced upon the plant being exposed to stress. In healthy plant tissue, they frequently occur at low levels, if detectable at all. Upon exposure to biotic or abiotic stress, the production of phytoalexins in the plant is induced and the levels are increased for them to play their role in plant defence. The biotic stressors are generally plant dependent but could, for example, be moulds and other microfungi. Among abiotic stressors, mechanical injury, type B ultraviolet light (UV-B), and ozone can be mentioned.

The speed and intensity of phytoalexin production is very important for a successful defence against pathogens (Pool et al., 1981). Also important are the types of compounds produced. In the well-studied grapevine (Vitis vinifera), it has been demonstrated that pathogen susceptible cultivars are able to produce high amounts of resveratrol that are rapidly converted into the more stable but less toxic resveratrol glucosides, whereas resistant cultivars are able to synthesise high amounts of resveratrol that is rapidly dimerised into the more toxic viniferins (Pezet et al., 2004; Schnee et al., 2008). A good correlation between stilbene production and resistance of grapevine to pathogens has been established (Stein \& Hoos, 1984; Sbaghi et al., 1995).

Figure 5: Molecular structure of the most common stilbenes in food. (A) trans-resveratrol, (B) transpiceid, (C), cis-resveratrol, and (D) cis-piceid<smiles>Oc1ccc(/C=C/c2cc(O)cc(O)c2)cc1</smiles>

(C)<smiles>Oc1ccc(/C=C\c2cc(O)cc(O)c2)cc1</smiles><smiles>OCC12COC(O1)C(O)C(O)C(O)C(O)C2O</smiles><smiles>OCC1COC(Oc2cc(O)cc(/C=C\c3ccc(O)cc3)c2)C(O)C1O</smiles> 
Stress regulation of stilbene synthesis in grapevine has been documented by stilbene synthase mRNA accumulating in two waves after the plant is exposed to cell walls of plant pathogens (Liswidowati et al., 1991; Wiese et al., 1994; Zinser et al., 2000; Borie et al., 2004; Douillet-Breuil et al., 1999). It has been hypothesised that these are waves dependent on two groups of stilbene synthase genes, those expressed early having a high turnover of the mRNA and those expressed later having more stable mRNA (Wiese et al., 1994). Further insight into the mode of induction and regulating pathways of stilbene production is given by Jeandet et al. (2010).

Table 34 summarises the stilbenes identified in food plants and gives reference to the original publication where this occurrence was described.

\subsubsection{Chemical analytical methodology}

The chemical properties of resveratrol and other stilbenes are of course important for chemical analysis. The solubility of resveratrol is low in water but good in organic solvents such as ethanol, acetone, chloroform, benzene, and acetic acid (Hart, 1981). The solubility increases with ethanol concentration and temperature (Filip et al., 2003). At room temperature and an alcohol concentration of $10 \%$, it is approximately $40 \mathrm{mg} / \mathrm{L}$. Resveratrol solutions are isomerised by light. Only 5 hours of exposure to diffuse daylight are required to reach an equilibrium between trans-resveratrol and cis-resveratrol from pure solutions of trans-resveratrol or cis-resveratrol (KolouchováHanzlíková et al., 2004). If protected from light, pure resveratrol solutions can be stored for one week at $20^{\circ} \mathrm{C}, 4^{\circ} \mathrm{C}$, or $-20^{\circ} \mathrm{C}$ without significant deterioration (Callemien et al., 2005). At temperatures from $30^{\circ} \mathrm{C}$ and higher, the trans-form of resveratrol was preferred to the cis-form (Kolouchová-Hanzliková et al., 2004).

In the presence of light, however, trans-resveratrol was very quickly degraded at room temperature, especially in the presence of oxygen. Similarly, Jerkovic and Collin (2008) demonstrated that aqueous solutions of trans-piceid are stable for two months at 4 and $25^{\circ} \mathrm{C}$ if protected from white light and oxygen. Appropriately stored, $76 \%$ were recovered after four months and $31 \%$ after twelve months. Not protected by the storage conditions, the glucoside was found to be unstable. Cis-resveratrol could be formed both from trans-resveratrol and cis-piceid.

Over time, many analytical techniques have been tested to identify and quantify stilbenes in plant tissues. Most recent methods are based on liquid chromatography (LC) (e.g. Zhu et al., 2000), HPLC (e.g., Jeandet et al., 1997; Revilla and Ryan, 2000; Piňeiro et al., 2006), or gas chromatography (GC), frequently coupled to mass spectrometry (MS) (e.g., Flamini, 2003; Flamini and Della Vedova, 2004). However, as derivatisation is required before $\mathrm{GC}$ analysis of the stilbenes to enhance volatility, and this is time consuming and may result in some trans- to cis- isomerisation of resveratrol to occur, the HPLC methods are preferred. 
Table 34: Stilbenes identified in food plants

\begin{tabular}{|c|c|c|}
\hline Trivial name & Chemical name and synonyms & Comments \\
\hline Trans-resveratrol & 3,5,4'-trihydroxy-trans-stilbene & $\begin{array}{l}\text { First isolated from the roots of the } \\
\text { white hellebore (Veratrum } \\
\text { grandiflorum) by Takaoka in } 1940 .\end{array}$ \\
\hline Cis-resveratrol & 3,5,4'-trihydroxy-cis-stilbene & \\
\hline Trans-piceid & $\begin{array}{l}\text { 3,5,4'-trihydroxystilbene-3-O- } \beta \text {-D- } \\
\text { glucopyranoside }\end{array}$ & $\begin{array}{l}\text { First isolated independently from } \\
\text { Eucalyptus vandoo (Hathway and }\end{array}$ \\
\hline & $\begin{array}{l}\text { Resveratrol 3-beta-mono-D-glucoside } \\
\text { Polydatin }\end{array}$ & $\begin{array}{l}\text { Seakins, 1959) and Picea glehnii } \\
\text { (Kariyone et al., 1958, 1959), the latter } \\
\text { explaining its trivial name, piceid. }\end{array}$ \\
\hline Cis-piceid & $\begin{array}{l}\text { 3,5,4'-trihydroxystilbene-3-O- } \beta \text {-D- } \\
\text { glucopyranoside } \\
\text { Resveratrol 3-beta-mono-D-glucoside } \\
\text { polydatin }\end{array}$ & \\
\hline Trans-astringin & $\begin{array}{l}\text { A } 3-O-\beta-D \text {-gluco(pyrano)side of } 3^{\prime} \text {-hydroxy- } \\
\text { resveratrol }\end{array}$ & \\
\hline Cis-astringin & $\begin{array}{l}\text { A } 3-O-\beta-D \text {-gluco(pyrano)side of } 3^{\prime} \text {-hydroxy- } \\
\text { resveratrol }\end{array}$ & \\
\hline Trans-resveratrol-oside & A 4'-O- $\beta$-D-gluco(pyrano)side of resveratrol & \\
\hline Cis-resveratrol-oside & $\mathrm{A}_{4}{ }^{\prime}-\mathrm{O}-\beta-\mathrm{D}-\mathrm{gluco}$ (pyrano)side of resveratrol & \\
\hline$\varepsilon$-viniferin & $\begin{array}{l}\text { (-)-Trans-epsilon-viniferin } \\
\text { (-)-Cis-epsilon-viniferin }\end{array}$ & Langcake and Pryce, $1977 \mathrm{~b}$ \\
\hline$\delta$-viniferin & $\begin{array}{l}\text { trans- } \delta \text {-viniferin } \\
\text { trans- } \delta \text {-viniferin }\end{array}$ & \\
\hline \multicolumn{3}{|l|}{$\alpha$-viniferin } \\
\hline Pallidol & A new resveratrol dimer & Via the bridging carbons \\
\hline Trans-pterostilbene & $3^{\prime}, 5^{\prime}$-dimethoxy-resveratrol & \\
\hline Piceatannol & $\begin{array}{l}3^{\prime}, 4^{\prime}, 3,5-\text { Tetrahydroxy-trans-stilbene } \\
\text { Astringinin }\end{array}$ & $\begin{array}{l}\text { First described as a component of the } \\
\text { heartwood extractabels of Vouacapoua } \\
\text { maropetala (King et al., 1956). }\end{array}$ \\
\hline Isorhapontigenin & $3,4^{\prime}, 5$-trihydroxy-3'-methoxystilbene & \\
\hline r-2-viniferin & & In Vitis species (Korhammer et al., 1995) \\
\hline
\end{tabular}

Soleas et al. (1997) compared four methods to measure the concentration of transand cis-resveratrol in wines. Two methods used solid-phase extraction before directinjection GC-MS or derivatisation before GC-MS, whereas the other two methods used direct-injection HPLC with gradient elution and DAD. The investigators identified a good correlation between methods ( $r>0.900$ ). However, some systematic bias was observed. High values for trans-resveratrol with direct-injection GC-MS could be attributed, at least in part, to thermal breakdown of resveratrol glucosides to free isomers. The derivatisation GC-MS technique showed a tendency to overestimate cisresveratrol and underestimate the trans-isomer, possibly as a consequence of trans- 
to cis-isomerisation during the derivatisation step. Somewhat lower values for cisresveratrol with the normal phase HPLC procedure might be a consequence of monitoring at a single wavelength $(306 \mathrm{~nm})$, which is well above the absorption maximum of this isomer. The reverse-phase gradient HPLC with diode array detection has the advantage of allowing the simultaneous quantitation of many other polyphenols of biological interest. Consequently, it may be considered the most robust method for routine application.

In addition to the standard analytical methodologies mentioned above, several investigators have used capillary electrophoresis of stilbenes and their glycosylated forms (e.g., Arce et al., 1998; Nevado et al., 1999; Gu et al., 2000; Brandolini et al., 2002; Vaher \& Koel, 2003).

Some investigators have optimised the chemical analytical method to analyse a particular stilbene. For example, Carando et al. (1999) described a chemical analytical approach to quantify trans-astringin in wine.

The methodology used to extract the compounds from the plant tissue, and clean-up the extract, is much more diverging and includes the task not to chemically modify the extracted compounds. A challenge when analysing stilbenes in products such as fruit juices, beer, grapes, and wine is to find an efficient pre-chromatography clean-up that would reduce the presence of interferences and result in good recovery of compounds (Lamuela-Raventos \& Waterhouse, 1993; Jerkovic et al., 2008; Viňas et al., 2008). Linked to this is the importance of the solvent for good recovery from the material and extraction equipment (e.g., Jerkovic et al., 2008). Several investigators have commonly used solid phase extraction methods (e.g. Shao et al., 2003) and even supercritical carbon dioxide (Casas et al., 2010).

As mentioned above, trans-resveratrol in solution is stable for months when protected from light, except in high pH buffers. Cis-Resveratrol, on the other hand, was stable only at near neutral $\mathrm{pH}$ when completely protected from light (Trela \& Waterhouse, 1996). The studies confirmed the original observation of Goldberg et al. (1995b) that light converts trans-resveratrol to the cis-form. However, it was shown that the wavelength was important. At $366 \mathrm{~nm}$, more than $90 \%$ of the transresveratrol were converted to the cis-form within 100 minutes. At $254 \mathrm{~nm}$, less than $63 \%$ were cis-resveratrol, even after 600 minutes of irradiation. The degradation by light has been studied in vitro (Silva et al., 2013). It was noted that trans-resveratrol is more easily degraded when irradiated with the whole spectral range (UV-Visible) than when irradiated with UV or near-UV to visible range. The main intermediate of transresveratrol phototransformation was identified as its isomer cis-resveratrol. The phototransformation of trans-resveratrol resulted in six photoproducts, mainly resulting from an attack of a hydroxyl radical to the molecule.

The reduced stability of stilbenes when exposed to light and the sensitivity of the molecules to oxidation in normal air had been observed already with earlier analytical methods (Hart, 1981). This sensitivity is probably at the origin of their denaturation observed using rotary evaporation (Pezet et al., 1994). In this system, when the solvent is evaporated, compounds are uniformly distributed as a thin layer on the wall of the flask and particularly exposed to air oxidation when vacuum is suddenly broken. 
It was concluded that all solvent evaporation steps must be done under nitrogen and protected from light.

\subsubsection{Stilbenes in nuts and almonds}

Optimisation of the extraction procedure before separation and identification of stilbenes is a challenge in peanuts (Chukwumah et al., 2007a). For example, resveratrol could be identified in methanol extracts of peanut skins but not in ethanol extracts (Ballard et al., 2009). There are also interfering compounds in the extracts that need to be removed before quantification.

Peanuts. Peanuts were early identified as prone to produce phytoalexins. Vidhyasekaran et al. (1972) studied the diffusates from immature and mature peanut pods (Arachis hypogaea) inoculated with five test fungi. Many species of microfungi are known to live on peanut pods (Joffee and Shiray, 1966). Whereas only one of the tested species was found to be established on immature pods, all species could endure on mature pods. Isolating the diffusates from immature pods and testing the diffusate on five fungal species, identified spore germination only for one of the strains, the one that had been found on the peanut pods. Nearly $100 \%$ of the spores germinated in sterile water. It was proposed that the diffusate from the peanut contained phytoalexins. Subsequent studies showed that also the diffusates of mature peanut contain phytoalexins but not as much as diffusates from immature pods.

It seems plausible that the production of phytoalexins by peanuts may interfere with colonising microfungi (such as Asperillus flavus) and their capacity to produce mycotoxins. However, abiotic stresses (e.g., drought) may break down the natural resistance mechanism supported by the phytoalexins, as indicated by studies of Dorner et al. (1989).

However, abiotic stress may also elicit phytoalexin production. Mechanical injury, such as that caused by slicing peanut seeds, was long ago recognised to elicit the synthesis of phytoalexins (Keen, 1975). The early studies on the nature of these phytoalexins revealed trans- and cis-resveratrol (32-44 and 6-11 mg/kg/L, respectively), trans- and cis-4-isopentenylresveratrol (3,5,4'-trihydroxy-4-isoprentenylstilbene), 4-(3methyl-but-1-enyl)-3,5,3',4' -tetrahydroxystilbene, and 4-(3-methyl-but-enyl)-3,5,4'trihydroxystilbene (Keen and Ingham, 1976; Ingham, 1976; Aguamah et al., 1981). Subsequent studies by Cooksey et al. (1988) identified 3-isopentadienyl-4, $3^{\prime}, 5^{\prime}$ trihydroxystilbene as the major antifungal component elicited by slicing imbibed kernels of peanut. Twenty-four hours after slicing, the level was between 38.5 and $105.8 \mathrm{mg} / \mathrm{kg}$ kernel. After 48 hours, the yield rose to between 89.5 and $189.7 \mathrm{mg} / \mathrm{kg}$ dependent on genotype. Sobolov et al. (1995) later demonstrated that the phytoalexins produced as a response to fungal invasion may be found in all parts of the peanut plant (leaves, stem, pegs, roots, kernels). However, as demonstrated by Mohanty et al. (1991), the phytoalexin profile in sliced seeds differed widely among twenty varieties studies. Thus, some genotypes produced one major phytoalexin component, whereas other genotypes produced several major components. 
Rudolf and Resurreccion (2005) noted an eightfold increase in trans-resveratrol concentration over time after exposure of peanut kernels to abiotic stress (size reduction by grinding, chopping, or slicing and exposure to UV light or ultrasound). Ultrasound was also shown to increase phytoalexin levels: trans-resveratrol from $0.03 \mathrm{mg} / \mathrm{kg}$ (control material) to $2.10-4.73 \mathrm{mg} / \mathrm{kg}$ and trans-piceid from 0.07 to 0.23 $0.38 \mathrm{mg} / \mathrm{kg}$ (Potrebko and Resurreccion, 2009). Also, UV light was shown to stimulate phytoalexin formation.

The early identification of resveratrol in peanuts was done on materials infected with microfungi, and these early reports indicated that the phytoalexin was not found in healthy material. However, some of the early data could be interpreted as phytoalexins occurring even in non-infected peanuts but at very low levels. Experimental support for phytoalexins being present appeared when Sanders et al. (2000) reported on resveratrol content in fifteen peanut cultivars. The analytical methodology allowed trans-resveratrol to be identified in all products. The level ranged from $0.022 \mathrm{mg} / \mathrm{kg}$ to $1.79 \mathrm{mg} / \mathrm{kg}$, with most cultivars containing levels below $0.13 \mathrm{mg} / \mathrm{kg}$. These investigators also reported that seed coats contained much higher levels of trans-resveratrol than the seed but because the weight of the seed coat is low, it did not influence the total trans-resveratrol content very much. Tokuşoğlu et al. (2005) studied six peanut cultivars from the local Turkish market for trans- and cisresveratrol and found mainly the trans-resveratrol compound, the level being between 0.03 and $1.92 \mathrm{mg} / \mathrm{kg}$.

Wang et al. (2013b) investigated the mini-core collection of peanut material (102 accessions representing $1 \%$ of the germplasm collection) in the USA for the potential to improve seed quality through genetic resources by measuring the variability of seed chemical composition. One of the parameters investigated was resveratrol content. The average content of resveratrol in healthy seeds was $0.10 \pm 0.043 \mathrm{mg} / \mathrm{kg}$ (range 0.03 to $0.26 \mathrm{mg} / \mathrm{kg}$ ). Further statistical analysis demonstrated a significant year effect and a year $x$ genotype interaction. The mini-core collection included seeds from four botanical varieties: A. fatigiata var. fastigiata (ff), A. fastigiata var peruviana (fp), A. fatigiata var vulgaris ( $\mathrm{fv}$ ), and A. hypogaea var hypogaea (hh), but no correlation was noted between variety and resveratrol content. There was an uneven distribution of the FAD 2A mutation. This mutation is particularly common in hypogaea varieties (33.82\%). It was noted that accessions homozygous for this mutant allele also had a significantly higher amount of resveratrol $(0.12 \mathrm{mg} / \mathrm{kg})$ than both the wild type and the heterozygous peanut varieties (both $0.09 \mathrm{mg} / \mathrm{kg}$ ).

Chukwumah et al. (2007b) evaluated the effect of processing (boiling, oil roasting, and dry roasting) on the trans-resveratrol content of peanuts. Trans-resveratrol was not detected in raw peanuts (with or without skin) but occurred at low levels $(0.9 \mathrm{mg} / \mathrm{kg}$ ) in thermally processed (boiled) Virginia peanuts. Commercially boiled peanuts contained even higher levels of trans-resveratrol $(14.92 \mathrm{mg} / \mathrm{kg})$. Edible peanuts are divided into four quality classes by the food industry (jumbos, mediums, splits, and number 1s) while inedible peanuts are classified into six classes. Sobolev and Cole (1999) demonstrated that the average amount of resveratrol was correlated to the peanut quality. Lowest levels were found in the edible jumbo and medium classes (about $0.070-0.078 \mathrm{mg} / \mathrm{kg}$ ), 
whereas the inedible peanuts contained $1.281-7.092 \mathrm{mg} / \mathrm{kg}$. Regarding peanut products, the lowest levels were found in roasted peanuts $(0.018-0.080 \mathrm{mg} / \mathrm{kg}$ ), whereas considerably higher levels were found peanut butter $(0.148-0.504 \mathrm{mg} / \mathrm{kg}$ ) and boiled peanuts $(1.779-7.092 \mathrm{mg} / \mathrm{kg})$. One explanation for this trend and the high amounts reported in boiled peanuts could be that the latter products contain more immature peanuts and that these contain higher levels of resveratrol (Vidhyasekaran et al., 1972; Dorner et al., 1989). Boiled peanuts also used material from damaged pods and because resveratrol is a phytoalexin, damaged material contain higher amounts of phytoalexins.

Pistachio. Seeds of pistachio, Pistacia vera L., are used in the confectionery industry and are consumed raw, sun-dried, or roasted. Unripe seeds, fresh ripe seeds, and dried ripe seeds of the variety Bianca were collected in Sicily, Italy and analysed for transresveratrol by HPLC (Ballistreri et al., 2009). The level of trans-resveratrol was reduced during seed ripening and drying from $17 \mathrm{mg} / \mathrm{kg} \mathrm{dw}$ in the unripe seeds to $12 \mathrm{mg} / \mathrm{kg}$ in the ripe seeds and $2 \mathrm{mg} / \mathrm{kg}$ in the sun-dried seeds. Tokuşoğlu et al. (2005) studied five pistachio cultivars from local markets in Turkey and found trans-resveratrol levels ranging from 0.09 to $1.67 \mathrm{mg} / \mathrm{kg}$. Even larger differences in the trans-resveratrol content (17-fold) between varieties of pistachio nuts were reported by Bolling et al. (2011). When Grippi and co-workers (2008) analysed 12 pistachio samples from Sicily, they detected very low amounts of trans- resveratrol $(0.07-0.18 \mathrm{mg} / \mathrm{kg}$ ) but quite high amounts of trans-piceid (6.20-8.15 mg/kg). Ballisteri et al. (2009) did not analyse for piceid.

Almond. Xie and Bolling (2014) analysed nine stilbenes in three varieties of California almond (Prunus dulcis). Polydatin was identified in almond ethanol extracts at a level of $0.072-0.085 \mathrm{mg} / \mathrm{kg}$ (at least $95 \%$ of which came from the skin) and piceatannol + oxyresveratrol in the blanching water $(0.002-0.026 \mathrm{mg} / \mathrm{kg})$.

\subsubsection{Stilbenes in hops and beer}

Jerkovic et al. (2005) analysed nine varieties of hops (Humulus lupulus) and identified trans- and cis-piceid and trans-resveratrol in all varieties. None of the varieties contained cis-resveratrol. The levels of trans-resveratrol were in the range $0.22-1.00$ $\mathrm{mg} / \mathrm{kg}$, trans-piceid in the range $4.15-8.89 \mathrm{mg} / \mathrm{kg}$ och cis-piceid in the range $2.30-6.01$ $\mathrm{mg} / \mathrm{kg}$. The total stilbene content was between 7.14 and $14.79 \mathrm{mg} / \mathrm{kg}$. The same research team followed up their investigations by quantifying the trans-resveratrol and trans-piceid content in American and European hop cone samples from 2004, 2005, and 2006 harvests (Jerkovic \& Collin, 2007). The samples from Europe came from Germany and the Czech Republic. As already observed in the earlier study, cisresveratrol was absent from the hop samples. The total trans-stilbene content was between 0.52 and $3.49 \mathrm{mg} / \mathrm{kg}$ in the German samples, between 0.16 and $6.09 \mathrm{mg} / \mathrm{kg}$ in the Czech samples, and between 2.91 and $11.66 \mathrm{mg} / \mathrm{kg}$ in the samples from the USA. The harvest year strongly influenced the stilbene content in the hops.

Because hops are not used directly after harvest for beer production but are dried and milled before storage, Jerkovic and Collin (2008), in another study, assessed the impact of conditions and storage on hop stilbene concentrations. In this study, six American hop varieties harvested in 2004 were analysed through storage in three 
conditioned forms, all under protected atmosphere (vacuum at $4^{\circ} \mathrm{C}$ ): leaf hop, derived pellets, and carbon dioxide extracts. Already, after a total storage of four months at $4^{\circ} \mathrm{C}$, a considerable reduction (39-88\%) in stilbene content of leaf hop was observed. In some samples, trans-piceid seemed more affected than its aglycone, perhaps because trans-resveratrol is partially regenerated from the glycoside (KolouchovaHanzlikova et al., 2004). At this point in time, cis-resveratrol was identified in all samples as were cis-piceid. Expressed in trans-resveratrol equivalents, up to $1.2 \mathrm{mg} / \mathrm{kg}$ cis-resveratrol was formed in Willamette hop cones over eight months at low temperature. Over the same period, $6.5 \mathrm{mg} / \mathrm{kg}$ of trans-stilbenes disappeared, indicating that other degradation products are formed. Studies showed that these were not the dimerisation products $\varepsilon$-viniferin or $\delta$-viniferin. Pelletisation induced strong stilbene degradation in some of the studied cultivars; however, after eight months of storage, the overall stilbene content is in the same range whatever the conditioning of storage.

Using modern biotechnology, Schwekendiek et al. (2007) transformed the Tettnang variety of Hop, which contains no stilbenes, with the grapevine stilbene synthase gene supplying a constitutive promoter ( $35 \mathrm{~S}$ cauliflower mosaic virus). The authors noted substantial quantities of resveratrol and its glycosylated derivatives in the transgenic plant. Piceid, the predominant derivative, reached a concentration of up to $560 \mathrm{mg} / \mathrm{kg} \mathrm{fw}$ in hop cones. This genetically modified product has not yet reached the European market.

Considering the occurrence of $1-10 \mathrm{mg} / \mathrm{kg}$ stilbenoids in hop and the requirement of close to $200 \mathrm{~g} / \mathrm{hL}$ in wort, an amount of no more than $2-20 \mu \mathrm{g} / \mathrm{L}$ stilbenoids could be expected in beer. Moreover, considerable degradation of trans-resveratrol is known to occur in the boiling kettle (60\% degradation after seven minutes of boiling), according to Jerkovic et al. (2005). Trans-Piceid is much more stable during heat treatment but it can be converted to trans-resveratrol by yeast enzymes through wort fermentation (Jerkovic et al., 2008).

Because hops have been found to contain trans- and cis-piceid and because hop pellets and hop cones contain trans- and cis-resveratrol (Callemien et al., 2005; Jerkovic \& Collin, 2007, 2008; Jerkovic et al., 2005, 2008; Schwekendiek et al., 2007), Chiva-Blanch and associates (2011) undertook an extensive survey of these constituents in beers obtained from various parts of the world. Of the 110 analysed beers, 79\% contained free resveratrol (59 beers contained trans-resveratrol: 3.68$66.74 \mu \mathrm{g} / \mathrm{L}, 69$ beers contained cis-resveratrol: $1.34-22.65 \mu \mathrm{g} / \mathrm{L}$ ), whereas only $33 \%$ of the beers analysed contained piceid ( 6 beers contained trans-piceid: $1.8-9.31 \mu \mathrm{g} / \mathrm{L}, 38$ beers contained cis-piceid: $1.80-24.24 \mu \mathrm{g} / \mathrm{L})$ in quantifiable amounts. The Belgian product Abbey beer ( $7.5 \%$ alcohol) had the highest total stilbene content with 66.74 $\mu \mathrm{g} / \mathrm{L}$ trans-resveratrol, $10.31 \mu \mathrm{g} / \mathrm{L}$ cis-resveratrol, and $4.17 \mu \mathrm{g} / \mathrm{L}$ cis-piceid. Because Jerkovic and co-workers (2005) had identified that hops contain lower contents of resveratrols than piceids and because beers contain higher amounts of resveratrols than piceids, it was speculated that resveratrol can be partly generated from its glucoside, although piceid in beer remains more stable during the brewing process than resveratrol (Tedesco et al., 2005). Alternatively, glycosides may be hydrolysed by 
yeast or bacterial $\beta$-glucosidase activity through beer fermentation (Callemien et al., 2005; Jerkovic et al., 2005). A third possibility could be that isomerases in the yeast convert trans- into cis-forms (Jeandet et al., 1995). Abbey beers, ale, weissbier, and stout beers contained significantly higher amounts of total resveratrol than lager and alcohol-free beers (Chiva-Blanch et al., 2011).

\subsubsection{Stilbenes in fruits and berries}

During the past ten years, several berries and fruits have been analysed for resveratrol and sometimes for other stilbenes. Most of the data available are presented in Tables 35 and 36. Screening Vaccinium berries collected in North America for resveratrol, pterostilbene, and piceatannol, Rimando et al. (2004) reported resveratrol to occur in all studied species but at levels significantly lower than in grapes. Berries from Canada contained significantly higher levels of resveratrol than berries from the USA, but it could not be established if this difference is real or due to different extraction methods having been used. Lingonberries obtained from Canada were found to have the highest content of resveratrol $(5.88 \mathrm{mg} / \mathrm{kg} \mathrm{dw})$, almost the same as that found in grape berries $(6.5 \mathrm{mg} / \mathrm{kg} \mathrm{dw})$. Pterostilbene and piceatannol were each found in only two of the five studied species and only in a few of the samples analysed. The levels of these two compounds were very low. Thus, pterostilbene was found in 3 of 65 samples, namely in the $V$. ashei cultivars Tifblue and Climax, and in a $V$. stamineum selection $\mathrm{SHF}_{3} \mathrm{~A}-2-108$ (99-520 $\mathrm{ng} / \mathrm{g} \mathrm{dw}$ ). Piceatannol was found in deerberry, highbush blueberry, and blackberry. Borowska et al. (2009) studied five cranberry cultivars grown in Poland and compared these varieties with wild common cranberry (Vaccinium oxycoccus). Fruit of wild common cranberry contained higher amounts of resveratrol (712 $\mu \mathrm{g} / \mathrm{kg}$ of fresh mass) than the cultivated cultivars (533-598) $\mu \mathrm{g} / \mathrm{kg}$ fw.

Rimando and Barney (2005) analysed resveratrol and naturally occurring analogues (piceatannol, pterostilbene) in nine Vaccinium species growing in the USA. Because berries were lyophilised after collection, the investigators presented the analytical data as $\mathrm{mg} / \mathrm{kg}$ lyophilised berries (dw). Resveratrol was found in seven of the nine species and pterostilbene in all species but in quantities lower than resveratrol. Piceatannol was analysed only in five species and found in three of those. Enzymatic hydrolysis of the extracts using $\beta$-D-glucosidase increased resveratrol levels up to 63 -fold, suggesting and demonstrating for the first time that resveratrol occurs as a glycoside in Vaccinium berries.

In their screening of 24 fruits for their content of resveratrol and piceid (trans- and cisforms of both compounds), Huang and Mazza (2011) detected low levels of particularly trans- and cis-piceids in many fruits. A few years earlier, Wang and co-workers (2007) reported on the occurrence of resveratrol in strawberries (0.69-1.48 mg/kg dw), the amount being influenced both by genetic and cultivation/environmental factors. It was noted that high temperatures during growth resulted in higher levels of resveratrol than when temperatures were lower. When the resveratrol content in seeds and pulp of the mature strawberry was compared, higher levels were found in the seed. Inclusion of compost in the soil increased resveratrol content of the harvested strawberries, as did preharvest application of methyl jasmonate, a known elicitor of phytoalexins. 
Table 35: The level of stilbenes ( $\mathrm{mg} / \mathrm{kg}$ dry weight*) in berries purchased or collected in the USA, Canada, or Estonia

\begin{tabular}{|c|c|c|c|c|c|c|c|}
\hline Matrix & Resveratrol & $\begin{array}{l}\text { trans- } \\
\text { resveratrol }\end{array}$ & $\begin{array}{l}\text { cis- } \\
\text { resveratrol }\end{array}$ & $\begin{array}{l}\text { trans- } \\
\text { piceid }\end{array}$ & cis-piceid & $\begin{array}{l}\text { Picea- } \\
\text { tannol }\end{array}$ & $\begin{array}{l}\text { Ptero- } \\
\text { stilbene }\end{array}$ \\
\hline $\begin{array}{l}\text { Alpine blueberry } \\
\text { (Vaccinium uliginosum) }\end{array}$ & $0.512-0.789$ & & & & & & $0.129-0.139$ \\
\hline $\begin{array}{l}\text { Bilberry } \\
\text { (Vaccinium myrtillus) }\end{array}$ & 0.77 & & & & & & \\
\hline $\begin{array}{l}\text { Black huckleberry } \\
\text { (Vaccinium } \\
\text { membranaceum) }\end{array}$ & n.d. -1.267 & & & & & n.d. -0.608 & $0.127-0.753$ \\
\hline Blackberry & & n.d. & 0.121 & 0.201 & 0.101 & & \\
\hline $\begin{array}{l}\text { Cranberry (Vaccinium } \\
\text { macrocarpon) }\end{array}$ & 0.90 & n.q. & n.d. & n.q. -0.182 & $0.229-0.275$ & & \\
\hline $\begin{array}{l}\text { Deerberry (Vaccinium } \\
\text { stamineum) }\end{array}$ & $0.047-0.503$ & & & & & n.d. -0.52 & n.d.-0.195 \\
\hline $\begin{array}{l}\text { Elliot's blueberry } \\
\text { (Vaccinium elliotti) }\end{array}$ & $0.406-0.453$ & & & & & n.d. & n.d. \\
\hline $\begin{array}{l}\text { Evergreen huckleberry } \\
\text { (Vaccinium ovatum) }\end{array}$ & $3.359-4.668$ & & & & & n.d. & $1.226-2.741$ \\
\hline $\begin{array}{l}\text { Half-highbush blueberry } \\
\text { (V. corymbosum } \times V \text {. } \\
\text { angustifolium) }\end{array}$ & $0.672-1.000$ & & & & & & $0.127-0.353$ \\
\hline $\begin{array}{l}\text { Highbush blueberry } \\
\text { (Vaccinium corymbosum) }\end{array}$ & $0.26-1.07$ & n.d. -140.0 & n.d. & & & $\begin{array}{l}0.186- \\
0.422\end{array}$ & n.d. -0.475 \\
\hline $\begin{array}{l}\text { Lowbush blueberry } \\
\text { (Vaccinium angustifolum) }\end{array}$ & 0.86 & $41.6-56.2$ & n.d. & & & & \\
\hline $\begin{array}{l}\text { Oval-leaved blueberry } \\
\text { (Vaccinium ovalifolium) }\end{array}$ & $1.923-2.116$ & & & & & 0.331 & n.d. -0.214 \\
\hline $\begin{array}{l}\text { Rabbiteye blueberry } \\
\text { (Vaccinium ashei) }\end{array}$ & $0.007-1.69$ & n.d. & n.d. & & & n.d. & $0.099-1.51$ \\
\hline Raspberry & & $\begin{array}{l}0.038- \\
0.058\end{array}$ & $0.001-0.008$ & $\begin{array}{l}0.212- \\
0.234\end{array}$ & $0.038-0.094$ & & \\
\hline $\begin{array}{l}\text { Red huckleberry } \\
\text { (Vaccinium parvifolium) }\end{array}$ & $3.560-4.668$ & & & & & n.d. & 1.212 \\
\hline Saskatoon berry frozen & & 0.021 & n.d. & 0.345 & 0.105 & & \\
\hline $\begin{array}{l}\text { Sea buckthorn } \\
\text { (Hippophae rhamnoides) }\end{array}$ & & n.q. & & & & & \\
\hline $\begin{array}{l}\text { Sparkleberry } \\
\text { (Vaccinium arboretum) }\end{array}$ & $0.125-0.519$ & & & & & n.d. & n.d. \\
\hline $\begin{array}{l}\text { Strawberry } \\
\text { (Fragaria } \times \text { ananassa) }\end{array}$ & $0.68-1.48$ & n.d. -1.94 & n.q. -0.0025 & n.d. -0.160 & $0.324-0.333$ & & \\
\hline Sweetheart sweet cherry & & n.d. & n.d. & 0.510 & 0.086 & & \\
\hline
\end{tabular}

Note: * $\quad$ mg/kg lyophilised berries are assumed to be equivalent to $\mathrm{mg} / \mathrm{kg}$ dry weight.

Source: Lyons et al., 2003; Rimando et al., 2004; Rimando \& Barney, 2005*; Gorbatsova et al., 2007; Wang et al., 2007; Huang \& Mazza, 2011. 
Table 36: The level of stilbenes $(\mathrm{mg} / \mathrm{kg}$ ) in alcoholic extracts of fruits purchased from the Canadian market and analysed by HPLC-MS/MS

\begin{tabular}{lllll} 
Fruit & trans-resveratrol & cis-resveratrol & trans-piceid & cis-piceid \\
Lapins sweet cherry & n.d. & n.d. & 0.609 & 0.047 \\
Apple & n.d. & n.d. & $0.295-0.705$ & $0.100-0.251$ \\
Apricot Prunus armeniaca* & $0.10-10.9$ & $0.10-10.9$ & & \\
Banana & n.d. & n.d. & n.d. & n.d. \\
Pear & n.d. & n.d. & $0.246-0.454$ & $0.029-0.048$ \\
Plum & $0.013-0.020$ & n.d. & $0.117-0.341$ & $0.059-0.137$ \\
Peach & n.d. & n.d. & 0.447 & 0.164 \\
Nectarine & n.d. & n.d. & 0.668 & 0.094 \\
Plantain (banana) & n.d. & n.d. & n.d. & n.d. \\
\hline
\end{tabular}

Note: * Data on resveratrol in alcohol extracts of 21 plum pox virus resistant apricot genotypes analysed by HPLC-UV-VIS (Sochor et al., 2010; Zitka et al., 2011).

Source: Huang \& Mazza, 2011.

Sochor et al. (2010) documented the resveratrol content of 21 new apricot (Prunus armeniaca) genotypes resistant against Plum pox virus and cultivated in Western Slovakia. The levels varied between 0.3 and $10.9 \mathrm{mg} / \mathrm{kg} \mathrm{fw}$, the average being 4.5 $\mathrm{mg} / \mathrm{kg} \mathrm{fw}$. In a subsequent study on three cultivars, resveratrol levels were in the range $0.096-2.256 \mathrm{mg} / \mathrm{L}$ (Zitka et al., 2011).

Brazilian investigators analysed resveratrol in pulp and by-products of twelve tropical fruits (pineapple, acerola, monbin, cashew apple, guava, soursop, papaya, mango, passion fruit, Surinam cherry, sapodilla, and tamarind pulp) from Brazil and identified the compound in by-products of guava ( 257 or $25.7 \mathrm{mg} / \mathrm{kg} \mathrm{dw}$ ) and Surinam cherry (1.025 or $103 \mathrm{~g} / \mathrm{kg} \mathrm{dw}$ ) (Ribeiro da Silva et al., 2014).

\subsubsection{Stilbenes in grape}

The role of stilbenes in the phytoalexin response of grapevine has been studied by many investigators. Biotic stresses in focus have been the microfungi usually infecting grape (Vitis) plants, such as grey mould (Botrytis cinerea), downy mildew (Plasmopara viticola), and Aspergillus carbonarius, as well as other ochratoxin-producing fungi. The abiotic stresses that have been investigated are mainly UV light exposure, ozone exposure, and climatic conditions.

Biotic stresses and natural differences in stilbene production

Langcake and Pryce (1976) identified trans-resveratrol as the major component responsible for the blue fluorescence of grapevine leaf tissue following fungal infection or exposure to UV light. The biosynthesis of this compound appears to be a non-specific response of members of the Vitaceae to infection or injury. The compound was not found in healthy leaves but accumulated to between 50 and 400 $\mathrm{mg} / \mathrm{kg} \mathrm{fw}$ in infected or UV-irradiated leaves and was found to be a major constituent (700 $\mathrm{mg} / \mathrm{kg}$ ) of lignified stem tissue. Shortly thereafter, the same investigators reported on the occurrence of viniferins ( $\alpha-, \gamma$-, and $\varepsilon$ - forms) in the Vitaceae family 
(Langcake and Pryce, 1977b). Studies on the reaction of grapevine to various microfungi have shown that the fluorescing compounds may be a part of the phytoalexin response of the grapevine.

Langcake and co-workers continued by studying the distribution of resveratrol in spreading lesions caused by the grey mould, Botrytis cinerea, on detached grapevine leaves (Langcake and McCarthy, 1979). Both the highest absolute amounts and the highest concentrations of resveratrol were found in the apparently healthy zone of tissue up to $5 \mathrm{~mm}$ in advance of the visibly rotted area. When the relationship between the concentrations of resveratrol around $B$. cinerea lesions on grapevine leaves was investigated in relation to susceptibility of those leaves to infection, an inverse relationship was observed.

In the studies that followed, Langcake (1981) investigated the relationship between the production of resveratrol, $\varepsilon$-viniferin, $\alpha$-viniferin, and pterostilbene and either the resistance of $V$. vinifera to the grapevine pathogen Botrytis cinerea or the resistance of Vitis riparia to the pathogens B. cinerea (grey mould) and Plasmopara viticola (downy mildew). Studies on the timing, local sites, and concentrations at which these stress metabolites accumulate in relation to growth of the pathogens suggest that these stress metabolites are responsible for inhibiting growth of the pathogens in resistance interaction. In the resistance of $V$. riparia, $\varepsilon$-viniferin was generally the predominant component, although $\alpha$-viniferin was produced in appreciable quantities. In the resistance of $V$. vinifera to $B$. cinerea, $\alpha$-viniferin predominated. Despite the relatively high antifungal activity of pterostilbene, this compound appeared to play no role in the resistance of either species.

Some microorganisms may be insensitive to stilbenes and grow in their presence, without modifying them. However, most microfungi must inactivate the stilbene to grow. Studies on grey mould (Botrytis cinerea) metabolism of stilbenes have revealed a laccase-like oxidative dimerisation, involving the hydroxyphenyl group situated at the 4'-position of the stilbene skeleton. The enzyme produces a resveratrol dehydrodimer called $\delta$-viniferin, which is slightly different from $\varepsilon$-viniferin. In addition, other oxidised resveratrol dimers, such as restrytisols (A-C), lechinol $F$, and pallidol, may be formed (Jeandet et al., 2010).

If stilbenes bind to specific enzymes or proteins, a specific molecular shape may be responsible for the antifungal activity associated with the compounds. The transisomer is planar while the cis-isomer is aplanar, with the latter consistently more toxic than the former. The difference in toxicity between the two geometric isomers suggests that the toxicity depends on a receptor site in the cells of sensitive fungi into which only stilbene molecules of a certain conformation can fit.

Upon fungal infection of grapevine leaves, trans-resveratrol is synthesised and converted into more toxic derivatives. In the grape berries, stilbene synthesis is found in the skin (Creasy \& Coffee, 1988; Jeandet et al., 1991) and has been primarily ascribed to fungal infection and other biotic stresses, or mechanical injury or other abiotic stresses (Jeandet et al., 1995; Adrian et al., 2000a; Romero-Perez et al., 2001). Gatto et al. (2008) investigated during three vintages the role of the grapevine genotype in determining (i) the concentration of resveratrols in the ripe berry, (ii) the 
influence of the ripening stage on the concentration of resveratrols in healthy grapes, and (iii) the molecular regulation of resveratrol synthesis during berry development. The average level of resveratrols (sum of trans-resveratrol, cis-piceid, and trans-piceid) in grapes of 78 red and white/pink varieties during the three-year survey was between 21 and less than $1 \mathrm{mg} / \mathrm{kg}$. Cis-Resveratrol was detected in only one of twenty varieties and did not occur $(0.37 \mathrm{mg} / \mathrm{kg})$ until the grape was ripe. The red varieties had on average a higher content than the white/pink ones. The differences among the varieties were higher than one order of magnitude, reaching a factor 30 for red grapes and 26 for white/pink grapes. Grapes in the Pinot family all ranked at the top level of stilbenes. The investigation clearly showed that the grapevine genotype plays a crucial role in determining the concentration of resveratrols in ripe grape. When the stilbene content was related to the ripening stage of the grapes, it was noted that the level of trans-resveratrol, trans-piceid, and cis-piceid in the grapes was increased from veraison (onset of ripening) to the ripe stage and even post-ripened stage. Another observation was that the high producers of stilbenes accumulated resveratrol preferentially in the glucosylated forms, trans- and cis-piceid, while the low resveratrol producers contained mainly the sugar-free form of resveratrol.

The level of resistance of different grapevine cultivars to Plasmopara viticola, from susceptible to highly resistant, were studied on grapes in vineyards and confirmed by the symptoms developed after inoculations. On the abaxial surface of infected leaves, $P$. viticola developed abundant sporangiophores on susceptible cultivars (Chasselas, Gamay, Gamaret, and Pinot Noir), whereas on less susceptible cultivars (Seyval Blanc and Johanniter) the parasite produced few sporangiophores and some necrotic spots at the site of infection. On resistant cultivars (Bronner, Solaris, IRAC 2091), P. viticola induced a hypersensitive response in which only necrotic spots were visible and the disease ceased to develop. Stilbenes were analysed 4, 7, 24 and 48 hours postinfection on small leaf samples cut from the site of infection (Pezet et al., 2004). Large differences were observed between the cultivars at $24-$ and 48 -hour time points. Susceptible grapevines produced resveratrol and its glycoside, piceid. In contrast, resistant plants produced high concentrations of $\varepsilon$ - and $\delta$-viniferin. Resveratrol and piceid have little or no toxic activity against $P$. viticola, whereas $\delta$-viniferin is highly toxic and can be considered an important marker for resistance of grapevine to downy mildew. The data indicate an importance of oxidative dimerisation of resveratrol in grapevine defence against $P$. viticola.

In studies on the interaction between Botrytis cinerea and grapevine, Bavaresco et al. (1997) inoculated field-grown grapes of the varieties Castor (interspecific crossing) and Huxelrebe ( $V$. vinifera crossing) with two strains of $B$. cinerea to study the response of berries to fungal infection with respect to the time course of phytoalexin (trans-resveratrol, $\varepsilon$-viniferin, and pterostilbene) accumulation and visual disease symptoms. The Botrytis-resistant variety Castor synthesised more stilbenes than the susceptible variety Huxelrebe (double the amount). The concentrations of the three compounds decreased from the first to the third stage of berry growth, indicating very low levels at ripening. Also, uninoculated berries produced stilbenes, although to a much lower extent than the inoculated ones. The trans-resveratrol and $\varepsilon$-vineferin 
accumulation was enhanced up to the fourth day after inoculation $(7.31 \mathrm{mg} / \mathrm{kg} \mathrm{fw}$ for trans-resveratrol and $11.69 \mathrm{mg} / \mathrm{kg}$ fw $\varepsilon$-for viniferin) and then dropped until day 16 . After two days, $\varepsilon$-viniferin levels were higher than trans-resveratrol levels. In infected berries, the amounts of $\varepsilon$-viniferin dominated over pterostilbene. A maximum of 0.25 $\mathrm{mg} / \mathrm{kg} \mathrm{fw}$ of pterostilbene was reached one day after inoculation.

Berries of the Vitis vinifera cultivar Barbera (disease susceptible) and the interspecific variety Castor (disease resistant) were infected at veraison by the ochratoxin-A producing fungus Aspergillus carbonarius to study the stilbene-synthase gene expression (Vezzulli et al. 2007b). Trans-resveratrol and piceatannol synthesis was also investigated. Fungus infection induced stilbene synthase gene expression and stilbenes were produced in both studied grapevine cultivars. However, expression and production were significantly higher in the Castor cultivar. Castor displayed few disease symptoms, whereas Barbera became highly infected.

Sbaghi et al. (1995) measured resveratrol phytoalexin levels in grapevine in vitro cultures to screen for grey mould (Botrytis cinerea) resistance. The ability of in vitro cultures from 13 Vitis species to produce resveratrol was studied after induction by UV light. The amount of resveratrol identified depended on species and variety. There was a good correlation between the resveratrol production by grapevine in in vitro cultures and grey mould resistance, except for two Vitis species for which no correlation was observed, which suggests that resistance of grapevines may sometimes be associated with factors other than stilbene phytoalexins.

Jeandet et al. (1995) studied the distribution of the phytoalexin resveratrol in limited lesions caused by Botrytis cinerea on ripe grape clusters in the vineyard. After attack of the pathogen, resveratrol synthesis is particularly strong in grape skin and low in fruit flesh. It occurs at an intermediate to lower level in the seeds. Resveratrol was predominantly present in the non-infected fruits close to the necrotic area. It was hypothesised that this localised response can help to arrest the spread of established $B$. cinerea lesions if climatic conditions are unfavourable to the pathogen. When conditions become favourable, i.e. after a mild and humid period, rapidly spreading lesions are observed on fruits despite the continued increasing production of resveratrol in the grapes. At this stage, resveratrol can reach three- to fivefold the values found before rot intensification. Resveratrol can be found at significant levels in the grape prior to any detectable lesions in fruits collected from apparently healthy clusters, which would suggest that the phytoalexin response is initiated soon after the recognition of the pathogen by the plant.

In 1980, Blaich and Bachmann showed that stilbenes (trans-resveratrol, including mono- and disaccharides, some polysaccharides, and $\mathrm{Cu}$ ions) are excreted by wounded grapevine leaves and showing biological activity down to $0.001 \%$. The inducing effect on stilbene production seems to be alginate and mucic acid, which are known to be metabolites of Botrytis cinerea growing on the Vitis plants. Some cellular compounds inhibit excretion of resveratrol, which is then accumulating in the leaf tissue at some distance around the wound margin. Within the cell, the compound is deposited within small areas of the cytoplasm or in the periplasm, probably near plasmodesmata. From there, it is distributed evenly into the cell walls. Studies in 
inverted microscope have shown that $90 \mu \mathrm{g}$ resveratrol $/ \mathrm{ml}$ reduced germination of Botrytis cinerea conidia by approximately 50\% (Adrian et al., 1997). The compound also resulted in cytological changes in cells of the hyphal tip (resulting in curved germ tubes), and significantly reduced mycelial growth of $B$. cinerea at concentrations ranging from 60 to $140 \mu \mathrm{g} / \mathrm{ml}$. A third of all the Botrytis cinerea conidia exposed to semi-lethal concentrations of resveratrol became discoloured, possibly as a result of laccase-mediated oxidation of resveratrol (Adrian et al., 1998).

Although there were no visual signs of Botrytis cinerea infection, two of three grape varieties studied by Adrian et al. (2000a) still showed resveratrol levels expected in an infected grape. UV exposure to such grapes (see further down) always induced the production of resveratrol, which is in contrast to grapes seriously affected by the $B$. cinerea infection. In the closest neighbourhood of a $B$. cinerea infection in a grape cluster, many berries were necrosed and resveratrol levels comparatively low (Adrian et al., 2000a). In zones further away, the resveratrol concentrations were 2-30-fold higher in UV-induced berries as compared with the non-induced berries. The highest levels were recorded in healthy UV-irradiated berries. For example, in the Chardonnay grape (white), the resveratrol concentration reached $33 \mathrm{mg} / \mathrm{kg}$ fw at 24 hours after UV irradiation, whereas levels were as high as $123 \mathrm{mg} / \mathrm{kg} \mathrm{fw} 48$ hours after irradiation. Thus, it is possible that "over-induced" berries are less responsive to phytoalexin induction, and consequently, may be more susceptible to pathogen attacks. The level of cis-piceid was much lower than the level of the trans-form. Whereas trans- $\varepsilon-$ viniferin was detected in two of the three grape varieties, no cis- $\varepsilon$-viniferin could be detected. In addition, no pterostilbene could be detected.

Similar observations were made by Pezet et al. (1991). When grapes affected to various degrees by Botrytis cinerea were analysed for resveratrol content, the level of the stilbene was unexpectedly higher in grapes having a lower degree of $B$. cinerea infection. This result could be explained if resveratrol formed in the $B$. cinerea-infected grapes might have undergone degradation by exocellular enzymes of the fungus (Pezet et al., 1991), a hypothesis that agrees with the observation that Sauterne wines are low in resveratrol (Lamuela-Raventos and Waterhouse, 1993). The resveratrol content is higher in infected grape berries that not yet had developed symptoms of infection. The highest levels were found in grapes affected about $10 \%$.

To study the pathogenicity of various isolates of Botrytis cinerea to grapevine, the ability of the pathogen isolated to degrade the stilbene phytoalexins resveratrol and pterostilbene was investigated by Sbaghi et al. (1996). All strains that degraded resveratrol and pterostilbene were highly or moderately pathogenic to in vitro cultures of grapevines after inoculation with agar disks containing mycelium, whereas those that were unable to degrade phytoalexins were non-pathogenic. In all cases, the hydroxystilbene degrading activity was related to the presence of laccase activity in the culture filtrates, as shown by using syringaldazine as substrate.

In line with these observations, incubating growing Botrytis cinerea with resveratrol in vitro resulted in the production of three new restrytisols $A-C$ and three oxidised resveratrol dimers (resveratrol trans-dehydrodimer, leachinol $\mathrm{F}$, and pallidol) (Cichewicz et al., 2000). Similarly, Breuil et al. (1998) demonstrated that resveratrol 
can be metabolised by a laccase-like stilbene oxidase of the grey mould. One of the metabolites formed was a resveratrol dehydrodimer.

De Rossi et al. (2012) investigated the influence of the grape variety on the growth of Aspergillus carbonarius on grapes as well as the correlation between the amount of ochratoxin $A$ and the content of trans-resveratrol produced after fungal contamination. Variations in the amount of ocratoxin A produced by the fungus are observed depending on both grape variety and on the induction of trans-resveratrol determined during the infection. It appears that the promptness of trans-resveratrol production is important for a successful defence against ochratoxin A production and therefore partly relies on the grape cultivar. If an increase in trans-resveratrol production in grape berries occurs early after the fungal infection, the berry exploits this compound to control ochratoxin A synthesis. If the increase in trans-resveratrol concentration were delayed after fungal infection, a control of ochratoxin A accumulation cannot he achieved.

Bavaresco et al. (2003) studied the effects of five ochratoxin A producing strains of Aspergillus on stilbene phytoalexin synthesis in Vitis vinifera grapes. The microfungi had varying influences on stilbenes in intact and damaged (punctured) berries. At veraison, trans-reservatrol levels remained low in intact berries but there was a several-fold increase in punctured berries. All but one of the tested fungi triggered trans-resveratrol production. In the damaged berries, the level of trans-resveratrol decreased during ripening, whereas it increased in intact berries. The increase in trans-resveratrol content continued for 18 days, whereas trans-piceid levels increased during 15 days and then declined. Piceatannol levels were quantifiable only at one time point during the study and only in grapes inoculated with one of the Aspergillus strains.

Li et al. (2006) studied the extractable amounts of resveratrol in berry skin and seeds in 120 Vitis grape germplasm cultivars during two consecutive years to determine the distribution of resveratrol among the main grape genotypes. For both years, rootstock cultivars obtained from hybrids of $V$. monticule $\times V$. riparia contained much higher amounts of resveratrol in skin and seed than all other grape genotypes studied, again demonstrating the importance of the genetic constitution. Cultivars of the European grapevine $(V$. vinifera) and their hybrids with $V$. labrusca had relatively low content of resveratrol in berry skin and seed (with a few exceptions, less than $2 \mathrm{mg} / \mathrm{kg}$ of skin or seed fw). Significantly higher amounts of resveratrol were found (1) in berry skin of seeded cultivars than in seedless cultivars, (2) in both berry skin and seeds of winemaking grapes than in table grapes, and (3) in red grapes than in green grapes.

It is likely that spontaneous resistance of Vitis species or cultivars are linked to a natural genotype-dependent high production of phytoalexins, whereas sensitive species or cultivars have lower production of these protective substances. To test this hypothesis, Douillet-Breuil et al. (1999) undertook a study determining the phytoalexin production (resveratrol, piceid, pterostilbene, and $\varepsilon$-viniferin) potential in leaves of three $B$. cinerea-resistant Vitis species ( $V$. rupestris, $V$. cinerea, and $V$. labrusca) and three cultivars of $B$. cinerea-sensitive $V$. vinifera. The elicitation potential of phytoalexins was studied using UV light as elicitor. Four phytoalexins were formed (trans-piceid, trans-resveratrol, trans- $\varepsilon$-viniferin, and trans-pterostilbene) and the 
maximum total concentration was reached $30-45$ hours after elicitation. The studies confirmed the hypothesis, demonstrating trans-resveratrol to occur at the highest level and cis-resveratrol levels to be below the limit of quantification. Two peaks of resveratrol synthesis were observed. The first peak in the leaves occurred after approximately 20 hours following elicitation and a larger peak appeared approximately 40 hours after elicitation. The time course for trans $\varepsilon$-viniferin production mirrored that of resveratrol but occurred at lower levels. Trans-piceid appeared at 10 hours after UV elicitation and maintained the level reached until 72 hours after elicitation. Pterostilbene synthesis was variable between the various species and genotypes. Only one genotype produced pterostilbene at a constantly high level.

Dercks and Creasy (1989) studied 17 Vitis species for their resistance to downy mildew (Plasmopara viticola) and noted that apart from one group of Vitis species (which exhibited resistance despite an inability to synthesise high concentrations of stilbene phytoalexins) resistance was correlated with the capacity to efficiently synthesise resveratrol and $\varepsilon$-viniferin following elicitation treatment with UV irradiation. The necrotic reaction of plant tissue after inoculation was correlated with phytoalexin accumulation rather than with reproduction of Plasmopara viticola. The phytoalexin response of the host was dependent on inoculum density. In three selected Vitis species in which the resistance was correlated with phytoalexin production, increased inoculum densities resulted in characteristic stilbene synthesis for each cultivar. The results also suggested that resveratrol is more important for the regulation of the Plasmopara viticola-grapevine interaction than $\varepsilon$-viniferin.

Wang et al. (2013a) studied the influence of the genetic constitution on the resveratrol content of the leaves and skin of grape berries of 75 grape cultivars (34 table grape hybrids, 15 table grapes of Vitis vinifera, 15 wine grapes of $V$. vinifera, 11 juice grapes, 2 wine hybrid grapes, and 5 rootstock grapes) belonging to three species and seven interspecific hybrids. Trans-resveratrol and trans- and cis-piceid were detected in berry skins and leaves, but cis-resveratrol was not. In most cultivars, total resveratrol (including the three compounds mentioned above) was higher in berry skins than in leaves. The resveratrol content depended on the genetic constitution of the grapevine. In berry skins of most cultivars and in leaves of almost all cultivars, cispiceid was the most abundant resveratrol; trans-resveratrol and trans-piceid were minor components. Some specific cultivars were found with extremely high levels of trans-resveratrol, cis-piceid, trans-piceid, or total resveratrol in berry skins or leaves. Rootstock cultivars had a higher content of total resveratrol than the other germplasm types. The cultivated European cultivars and their hybrids with $V$. labrusca had relatively low total resveratrol. There were no significant correlations of the amounts of total resveratrol or any individual resveratrol in berry skins and leaves. These data confirm that the genetic background is important for the amounts and types of stilbene produced in grapes.

Gene expression of five genes of the phenylpropanoid pathway known to be involved in stilbene biosynthesis ( $\mathrm{PAL},{ }_{4} \mathrm{CL}, \mathrm{C}_{4} \mathrm{H}$, StSy, and $\mathrm{CHS}$ ) was studied on pools of berries from low and high producers of resveratrol. It was noted that $P A L,{ }_{4} C L$, and 
StSy were expressed in much higher levels in the high producers of resveratrol than they were in the low producers and gene expression was increased from veraison to ripening. For StSy, it was more than thirty times higher in the high producers at veraison and remains higher but at a lower ratio. For $\mathrm{C}_{4} \mathrm{H}$ and $\mathrm{CHS}$, no significant difference in gene expression was noted. Studies of cDNA libraries of grape genes identified a set of differentially expressed CDNA clones in the high resveratrol producers as compared with the low producers at each developmental stage. Setting a cut-off value of 1.5 -fold change, 285, 154, and 289 cDNA clones were selected as being differentially expressed between high and low resveratrol producers at the veraison, ripening, and post-ripening phase, respectively. In general, more genes were found to be down-regulated in the high resveratrol producers than in the low ones. When gene function was allocated to the various genes (could be estimated for about $75 \%$ ), approximately $22 \%$ fell into the category "response to stimuli" (e.g., biotic and abiotic stimuli), 9\% "cell wall organisation", 8\% "transport", 5\% "transcription", $5 \%$ "carbohydrate metabolism", 4\% "protein metabolism", 5\% "signal transduction", $4 \%$ "developmental process", and $4 \%$ "secondary metabolic process". In the category "response to stimulus", the genes up-regulated in the high resveratrol producers were mainly involved in plant defence against fungal pathogens and in the general response to biotic stress (Gatto et al., 2008).

Studies of the stilbene composition of grapevine flowers have revealed different profiles in different cultivars (Keller et al., 2000). The role of the stilbenes in flowers might be to reduce primary infection of the flower, inhibiting an infection that otherwise might remain latent inside the developing berry until veraison.

\section{Abiotic stress}

UV light. Since stilbenes were identified as phytoalexins in the middle of the $1970 \mathrm{~s}$ (Langcake \& Pryce, 1976), studies have investigated the influence of UV light exposure on the stilbene content of grapes. In follow-up studies, Langcake and Pryce (1977a) demonstrated that the biosynthesis of resveratrol and viniferins by grapevine in response to UV irradiation shows a pronounced maximum in the wavelength region 260-270 nm, indicating that DNA is the photoreceptor for the response. This observation highlights the likelihood of well-regulated genetic machinery behind the stilbene elicitation.

Jeandet et al. (1991) compared the influence of UV light exposure of a red grape variety susceptible to grey mould (Vitis vinifera Pinot noir) with the influence of a similar exposure of a red grape variety (Vitis labrusca) tolerant to that mould on the stilbene content of the grapevine berries and leaves. When grapes were protected from light, they contained no cis-isomers of stilbenes; when exposed to light, the cisisomers appeared. At equivalent developmental stage, resveratrol levels were higher in leaves and berries of $V$. labrusca compared with $V$. vinifera. The UV exposure induced a considerable amount of resveratrol in leaves: $100-150 \mathrm{mg} / \mathrm{kg} \mathrm{fw}$ in $V$. vinifera and $350-440 \mathrm{mg} / \mathrm{kg} \mathrm{fw}$ in $V$. labrusca. Lower amounts were found in the berries but also in this case the level was higher in $V$. labrusca than in $V$. vinifera. To investigate whether resveratrol could be synthesised in the berries, the investigators 
UV-irradiated grape berries at various stages of maturity. When immature berries were irradiated and analysed for stilbene content, resveratrol could be detected in the skin but not in the fruit flesh in both Vitis species. The closer to maturity the UV dose was delivered, the less pronounced was the increase in stilbene level in the grapes. Jeandet et al. (1991) concluded that a decrease in the active defence mechanism of berries seems to take place during ripening.

In a series of studies, Cantos and co-workers analysed the influence of postharvest UV irradiation on stilbene content of grapes with the aim to determine whether post-harvest treatment of grapes allows elicitation of resveratrol (Cantos et al., 2000, 2001, 2002, 2003a). From these studies, data on the grape skin and grape flesh show that stilbenes are available only in the skin. Mature grapes not exposed to any post-harvest treatment contained all the three stilbenes analysed for - trans- and cis-resveratrol, and trans-piceid - but cis-resveratrol was only present in trace amounts. The total number of stilbenes was $2.4 \mathrm{mg} / \mathrm{kg} \mathrm{fw}$ (Cantos et al., 2000). Storage of the grapes at $0^{\circ} \mathrm{C}$ for ten days doubled the stilbene content. Studies of the skin of untreated grapes revealed about $10 \mathrm{mg} / \mathrm{kg}$ each of trans-resveratrol and transpiceid. UV irradiation of the grapes before storage resulted in elicitation of stilbenes, which was in contrast with the observations of Roggero and Garcia-Parrilla (1995), but these two studies employed very different UV doses.

In a study that followed, Cantos et al. (2003b) reported on the stilbene induction capacity of seven red wine grape varieties exposed to post-harvest UV-C irradiation. All the grape varieties increased their resveratrol, piceatannol, and viniferin content after UV-C treatment, except the grape variety Monastrell in which only piceatannol was induced. Other stilbenes, such as astringin (piceatannol glucoside) or piceid (resveratrol glucoside), were induced to a lesser extent and only in some varieties. The stilbene induction capacity of grapes submitted to UV-C irradiation did not necessarily match the final corresponding maximum stilbene content reached in each variety. The total stilbene induction capacity of the grapes ranged from 2.4-fold (in Merlot) to 10.9-fold (in both Cabernet Sauvignon and Cariňena) as compared with the untreated grapes (control). The total stilbene content after UV-C irradiation ranged from $11.6 \mathrm{mg} / \mathrm{L}$ in Monastrell to $35 \mathrm{mg} / \mathrm{L}$ in Merlot. In general, the maximum induction of specific stilbenes in all the varieties followed the sequence: resveratrol>piceatannol $>\varepsilon_{1}$-viniferin $>$ piceid. This maximum induction as compared with the control was 22.7-fold for resveratrol in Cabernet Sauvignon, 6.4-fold for piceatannol in Cariňena, 3.8-fold for piceid in Tempranillo, and 8.4-fold for $\varepsilon$-viniferin in both Cabernet Sauvignon and Cariňena. The maximum concentration of the specific stilbenes reached after UV-C irradiation was 25 $\mathrm{mg} / \mathrm{kg}$ fresh grape weight for resveratrol in Merlot, $4.2 \mathrm{mg} / \mathrm{kg}$ for piceatannol in Merlot, $1.6 \mathrm{mg} / \mathrm{kg}$ for piceid in Monastrell, and $3.9 \mathrm{mg} / \mathrm{kg}$ for $\varepsilon$-viniferin in Cariňena. A genotypedependent enhancement of stilbene production by post-harvest UV-C treatment in grapes has also been demonstrated by Guerrero et al. (2010).

Selma et al. (2008) investigated the efficacy of UV-C treatment (resulting in increased concentrations of stilbenes) in inhibiting Aspergillus carbonarius and $A$. tubingensis infection and controlling ochratoxin A production in grapes. UV-C exposure elicited the biosynthesis of trans-resveratrol while microbial infection and 
tissue damage triggered the biosynthesis of trans-piceid. Trans-resveratrol was not synthesised as a consequence of ochratoxigenic Aspergillus contamination. However, when trans-resveratrol was synthesised because of UV-C treatment of undamaged grapes and subsequently stored at $22^{\circ} \mathrm{C}$ for five days, development of ochratoxin Aproducing aspergillus was inhibited, resulting in reduced production of ochratoxin $\mathrm{A}$. The authors concluded that UV-C treatment of grapes shows promising attributes for reducing the potential ochratoxigenic risk in grapes, which is of particular interest for the wine industry. Moreover, UV-C treatment would increase the trans-resveratrol content of grapes, which would provide added value to the wine (health benefits).

Bais et al. (2000) studied the molecular regulation of stilbene phytoalexin biosynthesis in developing Vitis vinifera grape berries using a UV induction system. The berries were collected at $1,5,10$, and 16 weeks post-flowering from the cultivars Shiraz, Semillon, Cabernet Sauvignon, and Chardonnay, and their skins analysed for resveratrol production following elicitation by UV-C light irradiation. In all cultivars, the rate and maximal level of resveratrol accumulation were markedly increased in berries sampled 1-5 weeks post-flowering and then dramatically declined in maturing berries sampled 10-16 weeks post-flowering. In berries sampled at 1 and 5 weeks post-flowering, maximal levels or resveratrol accumulation was recorded at incubation periods of 24 and 48 hours after UV light exposure. Maximal resveratrol levels were not recorded in week 16 berry skins until 72 hours after UV treatment. Gene expression analysis indicated that stilbene synthase mRNA accumulated within 4-8 hours after UV treatment in berries sampled at 1 and 5 weeks post-flowering, but did not increase in week 16 berries until 24-48 hours following UV irradiation. Furthermore, the overall level of stilbene synthase gene expression declined in berries sampled 10-16 weeks post-flowering. These observations indicate that the expression of stilbene synthase influences stilbene accumulation. The decline in inducible stilbene synthase gene expression may be a major factor contributing to the increased susceptibility of ripening grape berries to Botrytis cinerea infection.

Ozone. Two studies have investigated the potential for post-harvest treatment of grape berries with ozone to increase their content of stilbenes. González-Barrio et al. (2006) noted that the content of trans-resveratrol, piceatannol, and viniferins (resveratrol dehydrodimers and dehydrotrimers) in the grapes reached maximum induction two days after ozone treatment, which is similar to that induced by UV-C treatment. Also, Sarig et al. (1996) observed resveratrol and pterostilbene to be elicited by ozone treatment. Whereas González-Barrio et al. (2006) found that ozone is particularly efficient in inducing viniferins in grape berries, Sarig et al. (1996) observed that resveratrol accumulated in greater quantities than pterostilbene. In a follow-up study, Sarig et al. (1997) demonstrated that maximum resveratrol accumulation in Rhizopus stolonifer-stressed grape berries was 4.0-8.5 times higher than that of pterostilbene, depending on Vitis vinifera cultivar. Both stilbenes reached highest concentration 24 hours after inoculation with $R$. stolonifer and thereafter levels declined gradually. Following UV-C radiation, resveratrol reached its highest concentration 18 hours after exposure and then declined rapidly thereafter. Pterostilbene reached its peak after approximately 40 hours. This was a general 
picture for the various cultivars studied. The higher amount of phytoalexins produced, the less severe was the decay from $R$. stolonifer. Phytoalexin accumulation declined with advancing maturity of the berries, coinciding with their increased susceptibility.

Based on the study referred to above, González-Barrio et al. (2006) proposed a sequence for the biosynthesis of phytoalexin stilbenoids, starting with the resveratrol monomer, continuing with the resveratrol dihydrodimers $\varepsilon$-viniferin and $\delta$-viniferin, and ending with four resveratrol dehydrotrimers. These trimers were different from $\alpha$ viniferin, a trimer previously reported to be induced in grapes after biotic and abiotic stresses (Langcake and Pryce, 1997b). In addition, the $\alpha$-viniferin isomers were detected in the ozone-treated grapes, although at very low concentrations that prevented their quantification.

To obtain further information on the molecular basis for one of the stress-induced plant responses, Grimmig et al. (1997) examined the promoter of the grapevine resveratrol synthase (Vst1) gene. In transgenic tobacco with a chimeric gene construct containing the Vst1 promoter combined with a $\beta$-glucuronidase (GUS) reporter gene, the signal from the reporter gene was rapidly induced by the stressor ozone. The same construct was also strongly induced by ethylene. Promoter deletion analysis of the $5^{\prime}$ flanking sequence identified a positive regulatory element between approximately 430 base pairs and -280 base pairs. The study identified a region containing an ethylene-responsive enhancer element, as well as an elicitor-responsive sequence in inverse orientation.

Jasmoate. Jasmoate is a well-known elicitor of phytoalexin response in plants (Wasternack, 2007). Larronde et al. (2003) demonstrated that comparatively low levels of methyl jasmonate could increase the UV-induced synthesis and accumulation of trans-resveratrol in grapevine berries. However, unlike their response to UV light, the berries rapidly lose their capacity to respond to methyl jasmonate during ripening. In 2007, Vezzulli and co-workers studied the effect of spraying methyl jasmonate on clusters of $V$. vinifera grapes during various stages of grapevine cultivation under drought stress conditions and regular water supply (Vezzulli et al., 2007a). Water stress had no influence on stilbene synthesis, but cumulative methyl jasmonate treatments during ripening significantly increased berry resveratrol and $\varepsilon$-viniferin production. Pre-harvest treatment of Syrah grapes with methyl jasmonate significantly increased their content of both trans-resveratrol (from non-detectable to $0.18-0.30 \mathrm{mg} / \mathrm{kg} \mathrm{fw}$ ) and piceatannol (from $0.74-1.16$ to $1.40-2.47 \mathrm{mg} / \mathrm{kg} \mathrm{fw}$ ). The concentration of these compounds peaked shortly after treatment and progressively decreased during ripening (Fernández-Marin et al., 2014).

\section{Stilbene content in grapes}

As already mentioned, stilbene production is constitutive in some parts of the grapevine plant and inducible in others. Whereas a large number of studies have analysed stilbenes in grape berries and wines, and some in the leaves of the grapevine (Vitis vinifera), only a few investigators have looked at the woody stem of the plant. Püssa et al. (2006) analysed three frost-hardy grapevine varieties [Hasaine (Hasansky) sladki, Ziga, and Yubilei Novgoroda] and identified 22 stilbenoids in the stem, most of 
them related to resveratrol ( $\mathrm{O}$ - and $\mathrm{C}$-glucosides, dimers, trimmers and tetramers, $\varepsilon$ viniferin, piceatannol, and its glucoside). The dominating compound was transresveratrol. Lambert et al. (2013), in addition, identified vitisin B and for the first time hopeaphenol and miyabenol $C$ in canes of Vitis vinifera. Although the stilbene expression in the woody part of the grapevine is constitutive, there is an annual fluctuation in resveratrol content of rhizomes and roots of grape plants, as demonstrated in V. amurensis by Ji et al. (2014). In China, there is a gradual increase in resveratrol content of these tissues from January to September and then a decrease until January of the following year.

In the green part of the plant, including the grapes, the situation is entirely different as stilbene production here is inducible and stilbene constituents are a part of the plant defence. The levels of stilbenes measured in leaves and grapes are therefore dependent on the health status and environmental conditions where the grapevine is grown. Comparing the level of resveratrol in the woody part of grapevine with the levels in the grapes, Ji et al. (2014) noted that during grape ripening the level in the grape skin is about half the level in roots and $40 \%$ of the level in the rhizome. The data of Király-Véghely et al. (1998), on the other hand, indicate that this relationship could be variety dependent. As already indicated, environmental factors have also been claimed to be important for stilbene production, including biotic interactions, climatic conditions, and fertilisation given to the grapevine plant.

The influence of climate on the stilbene content of grape berries has given contradictory information. Whether this is due to different grapevine varieties having been studied or environmental conditions, including the presence of phytoalexin eliciting pathogens being different at the various field trial sites in the studies, is not clear.

The largest study correlating climate and temperature with the stilbene content and stilbene profile of grapes was performed by Fernández-Marin et al. (2013). These investigators studied the stilbene content in four red grapes (Syrah, Merlot, Cabernet Sauvignon, and Pinot noir), all grown at four sites in Andalusia, Spain. Four stilbenes were found in the grape samples: piceatannol was detected in all varieties at two of the sites, in three varieties at a third site, and in none of the varieties at the fourth site. The other stilbenes detected were trans-resveratrol, $\varepsilon$-viniferin, and $\delta$-viniferin. Viniferins were only detected in a few samples. Resveratrol content was high at the geographical site having the highest average temperature and largest temperature range, combined with the lowest relative humidity average during the harvest period. The lowest resveratrol concentrations were found at another geographical site, which had the lowest temperature difference range and low average relative humidity.

Another study looking at the influence of climatic conditions (and possibly different degrees of fungal infection) on stilbene content of grapes studied Palomino fino grapes during two seasons (Roldán et al., 2003). In the first vintage, the total resveratrol content in the berry was higher by almost a factor of 2 than in the second vintage. The distribution of the different compounds in the berry also showed significant differences between the vintages. In the first vintage, there was a higher content of glucosides in the skin than in the second vintage. In the second vintage, the 
free and glycosylated isomers were present in greater quantities in the juice, although the total content of resveratrol was lower. There was a clear difference in climatic conditions between vintages (rainfall, temperature, radiation), with a higher stress in the first vintage. This might have induced the higher total resveratrol content during that season.

Reporting data on the grape variety, Monastrell, Moreno-Labanda et al. (2004) noted that the high resveratrol glucoside concentration and low free isomer content are typical of grapes growing in warm conditions. In contrast, other investigators found a relationship between low stilbene (trans-piceid, cis-piceid, trans-resveratrol, and cis-resveratrol) levels and higher temperatures or dry climate (Andrés-de Prado et al., 2007). Li et al. (2006) pointed out that rainfall during fruit development resulted in higher extractable amounts of resveratrol in berry skin, whereas resveratrol synthesis and accumulation in grape seeds were not related to climate change (Li et al., 2006). It seems likely that if climate has any significant contribution on the stilbene content of grapevine, this abiotic stressor will act in concert with biotic stressors, the presence of which is partly dependent on the climate.

Regarding fertilisation, there is some evidence that the supply of fertilisers (such as nitrogen and potassium) affects resveratrol synthesis of elicited leaves negatively (Bavaresco and Eibach, 1987; Bavaresco, 1993). In a pot experiment with the grape Cabernet Sauvignon, Bavaresco et al. (2001) compared the influence of supplying various dosages of nitrogen ( $0,4,8$, or $16 \mathrm{~g}$ per pot as ammonium nitrate) on the the level of trans-resveratrol in grape must obtained after harvest. The nitrogen fertilisation improved vegetative parameters and arginine content. Increased $\mathrm{N}$ rates decreased some quality parameters of the grape must, such as total soluble solids and trans-resveratrol concentrations, confirming the negative effect of high nitrogen supply already stated for leaves. It is believed that at a low supply of nitrogen the balance between the primary and secondary metabolic pathways might be shifted to the shikimate pathway, providing a large pool of phenolics and alkaloids. This pathway is the basis for physical and chemical defence mechanisms of plants, including phytoalexins.

Bavaresco and Eibach (1987) cultivated various varieties of grapevine cuttings in the greenhouse in pots at four levels of nitrogen supply to test their resistance to powdery and downy mildew and monitor their stilbene synthesis (resveratrol and $\varepsilon$ viniferin). Increased rates of nitrogen supply decreased the degree of resistance against powdery and downy mildew and reduced phytoalexin synthesis. Tested hybrids showed a higher powdery and downy mildew resistance and a higher stilbene synthesis than Vitis vinifera varieties (Riesling and Kerner).

López-Alfaro et al. (2013) measured the trans-resveratrol, cis-resveratrol, transpiceid, and cis-piceid content of grapes of the Graciano, Tempranillo, and Grenache cultivars. The Graciano cultivar contained significantly higher levels of these constituents than grapes of the other two varieties. Cis-resveratrol was not detected in any of the cultivars and trans-resveratrol only in the Graciano cultivar. In all three cultivars, trans-piceid was the most abundant stilbene, followed by cis-piceid. This result is expected as the glucosides do not need the help of ethanol to be efficiently extracted. 
Numerous investigators have analysed the stilbene content in grapes and various types of grape product. The available information is summarised in Table 37. In this table, green grapes are referred to as white grapes. The data clearly show that the stilbenes are not homogenously distributed in the grape. These compounds are more common in the skin and in the seeds than in the flesh of the grape. Such a distribution will influence the content in products such as juice, must, pomace, and pulp. Table 37 also indicates that red grapes are richer in stilbenes than white grapes.

Ector et al. (1996) studied the occurrence of resveratrol in muscadine (Vitis rotundifolia) grapes, juice, pomace, purees, seeds, and wines. Both dark- and bronzeskinned puscadines were studied. Dark-skinned berries contained slightly higher levels of resveratrol than brown-skinned puscadines but the difference was statistically different only for pomace. Levels in whole berries were $8.61 \mathrm{mg} / \mathrm{kg}$ for the bronzeskinned and $14.81 \mathrm{mg} / \mathrm{kg}$ for the dark-skinned. Most resveratrol occurred in the seeds $(43.7-44.6 \mathrm{mg} / \mathrm{kg})$. Berries without seeds contained $6.39 \mathrm{mg} / \mathrm{kg}$ for bronze-skinned berries and $12.26 \mathrm{mg} / \mathrm{kg}$ for dark-skinned berries. The investigators also studied the resveratrol content of various muscadine products (results in dark-skinned is given; bronze-skinned is given within brackets). Berries without seeds contained $11.73 \mathrm{mg} / \mathrm{kg}$ (9.30), juice $7.12 \mathrm{mg} / \mathrm{L}$ (7.91), pomace $53.21 \mathrm{mg} / \mathrm{kg}$ (33.69), puree $35.25 \mathrm{mg} / \mathrm{kg}$ (29.56), and wine $1.14 \mathrm{mg} / \mathrm{L}(0.67)$.

Skin. Using an optimised extraction method, Romero-Pérez et al. (2001) studied seven grape varieties (a mix of red and white grapes) for stilbene content in the skin. On average, they contained 92.33 mg cis-piceid/kg dry skin, $42.19 \mathrm{mg} / \mathrm{kg}$ trans-piceid/kg dry skin and $24.06 \mathrm{mg}$ trans-resveratrol/kg dry skin. No cis-resveratrol was detected in the skin. These data agree fairly well with those reported in Table 37. In grape berries infected by powdery mildew (caused by the fungus Uncinula necator), the content of these compounds was considerably increased and the degree of infection was positively related to their stilbene content. Particularly high resveratrol and piceid levels were observed in Merlot (red grape) and Zarel-lo grapes (white grape). There was no major difference in trans-resveratrol levels in the skin of red and white grapes in this study.

In a study investigating the level of trans- and cis-resveratrol, trans-piceid, and astringin (trans-3,4,3',5'-tetrahydroxystilbene-3'-O- $\beta$-D-glucopyranoside) in grape skin extracts of Croatian grapes, Katalinic et al. (2010) noted that the average contents of resveratrol monomers in grape skin of red grape varieties were almost three times as high as in the skin of white grape varieties. There was no significant difference in the level of trans-piceid and astringin between these two types of grape. The level of trans-piceid was below the detection limit in two of twelve grape varieties and the astringin level below the detection limit in one of the varieties. The latter compound, previously found in grape leaves and stems, on average occurred at $0.53 \mathrm{mg} / \mathrm{kg} \mathrm{fw}$ in red grapes and $0.60 \mathrm{mg} / \mathrm{kg} \mathrm{fw}$ in white grapes. The studies indicate that the polyphenol spectrum in grapes, including that of stilbenes, is variety dependent. This was confirmed by Chafer et al. (2005), who compared the trans-resveratrol content in grape skin and noted that the level in the grape variety Tempranillo was considerably higher $(174.0 \mathrm{mg} / \mathrm{kg}$ ) than in the varieties Tinto mozueleo, Cabernet, Boval, and Merlot (21.5-80.3 mg/kg). 
Table 37: Stilbene content in grapes and in various types of grape product

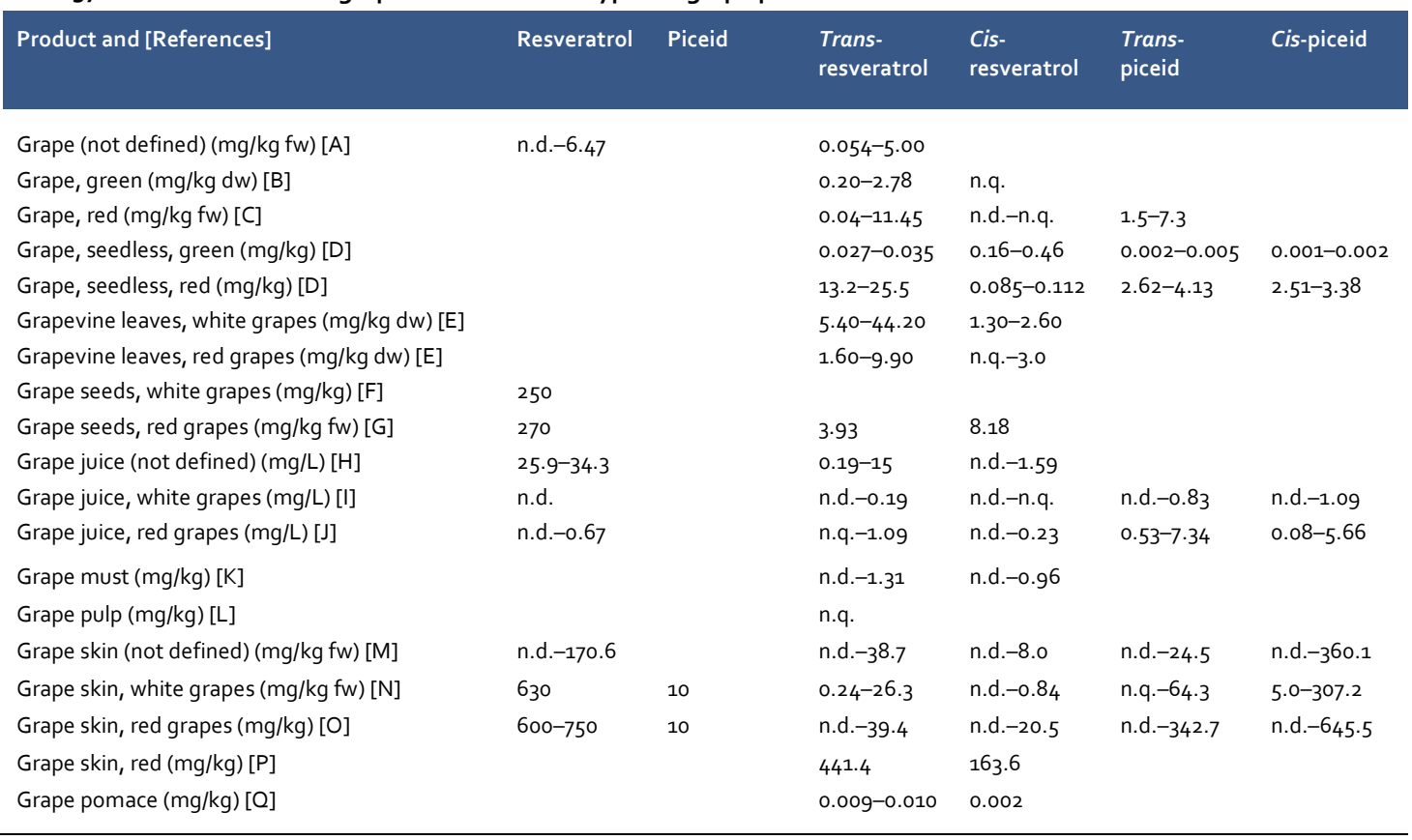

Note: $\quad$ n.d. = non-detectable; n.q. = non-quantifiable.

Source: [A]: Bavaresco et al., 1997; Palomino et al. 2000; Pastrana-Bonilla et al. 2003; Rimando et al., 2004; Pineiro et al., 2006; [B]: Melzoch et al., 2001; Pineiro et al., 2006; [C]: Burns et al., 2002: Cantos et al., 2003a; Pineiro et al., 2006; FernándezMarin et al. 2013; [D]: Huang \& Mazza, 2011; [E]: Melzoch et al., 2001; [F]: Melzoch et al., 2001; [G]: Pezet \& Cuenat, 1996; Counet et al., 2006; [H]: Soleas et al., 1995a; Sautter et al., 2005; de Freitas et al., 2009; Lima et al., 2014; [I]: Romero-Pérez et al., 1999; Natividade et al., 2013; [J]: Romero-Pérez et al., 1999; Viňas et al., 2008; Natividade et al., 2013; [K]: RomeroPérez et al., 1999; Viňas et al., 2008; Natividade et al., 2013: [L]: Palomino et al. 2000; [M]: Soleas et al., 1995a; Pezet \& Cuenat, 1996; Palomino et al. 2000; Pascual-Marti et al. 2001; Pastrana-Bonilla et al. 2003; Liu et al., 2013; Wang et al., 2013a; [N]: Adrian et al., 2000a; Romero-Pérez et al., 2001; Counet et al., 2006; Kataliníc et al., 2010; [O]: Adrian et al., 2000a; Cantos et al., 2000; Romero-Pérez et al., 2001; Artés-Hernández et al., 2003; Careri et al., 2004; Counet et al., 2006; Kataliníc et al., 2010; [P]: De Nisco et al., 2013; [Q]: Careri et al., 2004.

\subsubsection{Stilbenes in grape juice}

The data in Table 37 illustrate that juice produced from red grapes contains more stilbenes than juice produced from white grapes. Grape juices were investigated for their stilbene content by Pour Nikfardjam et al. (2000). In a commercial juice from white grapes made from a mix of berries, only low amounts of resveratrol-derivatives were found, $0.5 \mathrm{mg} / \mathrm{L}$ (n.d. $-2.2 \mathrm{mg} / \mathrm{L}$ ), whereas levels were higher in a juice from red grapes, $3.1 \mathrm{mg} / \mathrm{L}$ (n.d.-12.5 mg/L). Another juice made from a single white grape cultivar contained, on average, $0.2 \mathrm{mg}$ resveratrol/L (n.d. $-0.5 \mathrm{mg} / \mathrm{L}$ ), whereas juices produced from individual red grape varieties contained higher levels of resveratrol, $6.3 \mathrm{mg} / \mathrm{L}$ (n.d. $-15.3 \mathrm{mg} / \mathrm{L}$ ). Wang et al. (2002b) reported that a litre of grape juice contains $1.13 \mu \mathrm{mol} / \mathrm{kg}$ trans-resveratrol and $0.43 \mu \mathrm{mol} / \mathrm{kg}$ cis-resveratrol. Also, raw cranberry juice contained more of the trans-form than the cis-form (0.93 $\mu \mathrm{mol} / \mathrm{kg}$ trans-resveratrol and $0.14 \mu \mathrm{mol} / \mathrm{kg}$ cis-resveratrol). The resveratrol-content was found 
to depend on the grape variety, as reported for wine (Pour Nikfardjam et al., 2000). Romero-Pérez et al. (1999) noted that piceids are more common in grape juices than resveratrol and trans-isomers more common than cis-isomers.

One of the factors that might influence stilbene levels in grape juice is the method of producing the grape juice, both with respect to the method of agriculture and the method of juice production. Dani et al. (2007) produced grape juices from organically or conventionally grown grapes of Vitis labrusca and reported juices from organically grown grapes to contain higher resveratrol contents than juice from conventionally grown grapes (18-21 mg/L resveratrol as compared with about $8 \mathrm{mg} / \mathrm{L}$ resveratrol). The difference could possibly be explained by different loads of pathogen exposure in the two types of growing system.

Also, the method of producing juices from grapes might influence stilbene levels in the juice. Higher stilbene contents were found in hot pressed juices than in cold pressed juices (Leblanc et al., 2008). Other production factors having been recognised to influence levels in the final juice is whether grapes had been frozen and thawed before juice production and whether grapes were exposed to pectolytic enzymes before pressing.

\subsubsection{Stilbenes in wine}

A large number of studies have investigated whether stilbenes occur in grapevine berries and in wine and what their levels are, as well as the various factors that influence these levels. The stilbenes identified in wine include, additional to the wellstudied aglycones trans- and cis-resveratrol, their corresponding 3-O- $\beta$-Dglucosylated derivatives trans- and cis-piceid, a few resveratrol dimers ( $\varepsilon$-viniferin, $\delta$ viniferin) and trimers ( $\alpha$-viniferin), but also a few less frequently studied stilbene derivatives. The latter are mono-stilbene 2,4,6-trihydroxyphenanthrene-2-Oglucoside, two isomeric resveratrol-2-C-glucosides, four dimeric stilbenes glucosides (cis- and trans- $\varepsilon$-viniferin diglucoside, pallidol glucoside, and pallidol diglucoside), and trans-astringin (3,5, ', $4^{\prime}$-tetrahydroxystilbene-3-O- $\beta$-glucoside) (Goldberg et al., 1995b; Ribeiro de Lima et al., 1999; Baderschneider and Winterhalter, 2000). It is likely that some of these compounds occur only in grapes (and therefore in wine) that have been attacked by plant pathogens, resulting in a phytoalexin response in the grapevine. Another possibility is that some of the compounds are formed during the fermentation step of wine production, possibly being metabolites produced by microorganisms. The majority of available studies on stilbene derivatives in wine have focused on a single or a few of these constituents, usually the isomeric forms of resveratrol and piceid. This apparently limits the conclusions that can be drawn about total stilbenes in wine. 
Table 38 summarises the reported data on stilbene content in wine from various regions of the world. The Table gives the common range of trans-resveratrol, cisresveratrol, trans-piceid, and cis-piceid reported in wines, sometimes complemented with the highest deviating value given within brackets. It is evident from the table that red wine contains higher levels of these constituents than white wines. Regarding resveratrols, this has been documented by a large number of investigators (Mattivi, 1993a, 1993b; Fregoni et al., 1994; Goldberg et al., 1995c; Dourtoglou et al., 1999; McMurtrey et al., 1994; Ribeiro de Lima et al., 1999; Luan et al., 2000, Lee and Rennaker, 2007, Adrian et al., 2000; Vrhovsek et al., 1995). Rosé wines on average had slightly higher amounts of these compounds compared with white wines (RomeroPérez et al., 1996b), and lower than red wines (Mattivi, 1993b; Sun et al., 2003; Abril et al., 2005). These differences are largely explained by oenological practices during wine production, especially the skin contact time during maceration as previously indicated by Siemann and Creasy (1992). Presenting typical mean levels of these constituents is extremely difficult as levels are influenced by many factors, including grape variety used for the wine production, its history of exposure to grapevine pathogens and other stressors, climatic and other environmental conditions during growth, and the method of producing the wine. The influence of these and other factors on stilbene levels in wine has been demonstrated by others (Stervbo et al., 2007) and are discussed in more detail later in the chapter.

A common method to analyse these constituents in wines is to inject the wine directly into the HPLC after a simple clean-up step and then identify and quantify the constituents by various methods. This procedure, however, is not entirely straightforward. For example, Lamuela-Raventos et al. (1995) recognised that the analytical method is partly responsible for the differences in stilbene content noted in wine by various investigators. A challenge when analysing stilbenes in products such as grape juice and wine is to find an efficient pre-chromatography clean-up that reduces the presence of interference (Lamuela-Raventos \& Waterhouse, 1993). 
Table 38: The content $(\mathrm{mg} / \mathrm{L})$ of the most common stilbenes in various types of wine

$\begin{array}{lllllll}\text { Wine } & \text { Trans-resveratrol } & \text { Cis-resveratrol } & \text { Trans-piceid } & \text { Cis-piceid } & \text { References } \\ \text { Red wine } & \text { traces-11.9 (36.13) } & \text { traces-9.76 } & \text { n.d.-50.8 } & \text { n.d.-24.1 } & \text { A } \\ \text { Rosé wine } & 0.01-1.76 & \text { n.d.-1.56 } & \text { n.d.-0.91 } & \text { n.d.-2.25 } & \text { B } \\ \text { White wine } & \text { n.d.-0.66(7.95) } & \text { n.d.-1.7 } & \text { n.d.-16.10 } & \text { n.d.-3.34 } & \text { C } \\ \text { Wine } & \text { n.d.-14.32 } & \text { traces-2.31 } & 0.15-3.59 & 0.10-2.90 & \text { D } \\ \text { Wine, from botrytised grapes } & \text { traces-0.5 } & \text { traces-7.8 } & \text { traces-3.4 } & \text { traces-2.9 } & \text { E }\end{array}$

Note: n.d. = non-detectable levels; n.q. = non-quantifiable levels.

Source: A: Mattivi, 1993b; Pezet et al., 1994; Goldberg et al., 1995b, 1996a, b; Jeandet et al., 1995; LamuelaRaventos et al., 1995; Soleas et al., 1995a; Vrhovsek et al., 1995; Sato et al., 1997; Chu et al. 1998; Ribeiro de Lima et al., 1999; Dourtoglou et al., 1999; Gu et al., 1999; Adrian et al., 200ob; Burns et al., 2000; Viňas et al., 2000, 2008; Baptista et al., 2001; Kallithraka et al., 2001; López et al., 2001; Malovaná et al., 2001; Melzoch et al., 2001; Sakkiadi et al., 2001; Rodriguez-Delgado et al., 2001, 2002; Souto et al., 2001; Burns et al., 2002; Gao et al., 2002; Vitrac et al., 2002; Careri et al., 2003, 2004; Poussier et al., 2003; Shao et al., 2003; Sun et al., 2003; Gambelli \& Santaroni, 2004; Gobbi et al., 2004; Kolouchová-Hanzliková et al. 2004; Moreno-Labanda et al., 2004; Abril et al., 2005; Padilla et al., 2005; Gerogiannaki-Chrisopoulou et al., 2006 ; Pour Nikfardjam et al., 2006b; Andrésde Prado et al., 2007; Gambuti et al. 2007; Gürbüz et al., 2007; Lee \& Rennaker, 2007; Perrone et al., 2007; Dekic et al., 2008; Feijóo et al., 2008; Cai et al., 2009; Vlase et al., 2009; Cvejic et al., 2010; Lucena et al., 2010; Loizzo et al., 2011; Paulo et al. 2011; Pérez-Trujillo et al., 2011; Atanackovíc et al., 2012; Kostadinovic et al., 2012; Nour et al., 2012; De Nisco et al., 2013; Fernández-Marin et al., 2014; B: Mattivi, 1993b; Romero-Pérez et al., 1996b; Sato et al., 1997; Pozo-Bayón et al., 2003; Sun et al., 2003; Abril et al., 2005; Dekic et al., 2008; C: Pezet et al., 1994; Goldberg et al., 1995C; Sato et al., 1997; Pozo-Bayón et al., 2003; Sun et al., 2003; Viňas et al., 2008; Cvejic et al., 2010; D:

Lamuela-Raventos \& Waterhouse, 1993; McMurtrey et al., 1994; Roggero \& Garcia-Parrilla, 1995; Goldberg et al. 1996a; Lamikanra et al., 1996; Nevado et al., 1999; Cantos et al., 2003a; Careri et al., 2004; Flamini \& Dalla Vedova, 2004; Gambuti et al. 2004; Mark et al., 2005; Avar et al., 2007; Li et al., 2011; Figueiredo-González et al., 2014; E: Pour Nikfardjam et al. 2006a.

The focus in stilbene analysis in early studies was on resveratrol in that this compound could contribute to the French paradox, an observed inverse relationship between moderate wine consumption and risk of coronary heart disease (Siemann \& Creasy, 1992). Several studies made no distinction between trans- and cis-resveratrol. In these studies, the level of resveratrol was usually reported to be below $8 \mathrm{mg} / \mathrm{L}$ in red wine (Jeandet et al., 1993; Mattivi, 1993b; Roggero \& Archier, 1994; Celotti et al., 1996; Király-Véghely et al., 1998; Alonso et al., 2002; Woraratphoka et al., 2007; Yoo et al., 2011), with two deviating reports on higher levels. One report by Flamini and Della Vedova (2004) analysed three types of wine and reported total free resveratrol contents in wines between 11.8 and $17.2 \mathrm{mg} / \mathrm{L}$. In the other report, Alonso et al. (2002) reported that double-macerated red wines contained $18.56-18.92 \mathrm{mg}$ resveratrol/L as compared with non-detectable levels to $5.84 \mathrm{mg} / \mathrm{L}$ in single-macerated red wines. Among the 21 grape varieties from 18 regions studied by Stervbo et al. (2007), the lowest average level of trans-resveratrol was found in wines from the Zinfandel variety grown in the USA.

In subsequent studies, most researchers have analysed both trans- and cisisomers, not only of the aglycone resveratrol but also of its $3-O-\beta$-D-glucosylated form, better known as trans- and cis-piceid. Comparing the stilbene profile of wines 
with that of grape skin, from which the majority are derived, indicates that some aglycones are produced during wine production, either from the piceids or from dimeric or trimeric forms of resveratrol. When comparing wines made from the thinskinned grapes with wines made from the thick-skinned grapes, Stervbo et al. (2007) found no trend suggesting that the thickness of the grape skin correlates to the content of trans-resveratrol in the red wine.

Cis-resveratrol is not present in any significant amount in grape skin or grape juice but it appears to be formed by hydrolysis of piceid or resveratrol polymers (viniferins) or possibly by light-induced or enzymatic isomerisation of trans-resveratrol during skin fermentation. Thus, Roggero and Garcia-Parrilla (1995) found that cis- and transresveratrol contents increased and glycoside contents decreased during wine must (the freshly pressed grape juice containing skins, seeds, and stems) fermentation. Experimental studies have shown that it takes approximately 9 hours for wine stilbene O-glycosides to be hydrolysed after incubation with $\beta$-glucosidase at $50^{\circ} \mathrm{C}$ (La Torre et al., 2004). This type of enzyme is expressed by several of the microbes present during wine making. An additional possibility would be isomerisation due to light exposure of wine during the winemaking process, or possibly from light exposure of wine bottles during storage (Lamuela-Raventos et al., 1995). Jeandet et al. (1995a) studied the ratio of trans-resveratrol to cis-resveratrol in grapes and wine and noted only small amounts of the cis-isomer in the skin. In wine, both forms were found. The percentage being the cis-isomer in wine was $41 \%$ in wines vinted in the dark and $46 \%$ in wines not protected from light.

Lucena et al. (2008) noted that (red) wines from the Sāo Francisco Valley in Northeastern Brazil contain significantly higher concentrations of cis-resveratrol than trans-resveratrol. The investigators speculated that this could be due to the specific grape varieties in combination with the enological techniques (restricting water to the grapes after veraison) used in wine-making in the region. In most wines, transresveratrol levels are higher than cis-resveratrol levels, but these levels may be higher or lower than the level of trans- and cis-piceid. Goldberg et al. (1996b, 1996c) assayed more than 700 commercial red wines from most of the wine-producing areas of the world for the isomers of resveratrol and piceid. The highest levels of piceid were found in wines from France, Canada, Italy, Spain, Portugal, and South America. In the last three regions, the piceid concentrations exceeded those of resveratrol, suggesting that the high sugar content of warm climate grapes, or increased pathogen pressure under these conditions may favour the glycosylation of resveratrol. In nine regions, the concentration of cis-piceid exceeded that of trans-piceid, the reverse being the case in five, yet in all wines trans-resveratrol concentrations exceeded those of cisresveratrol. The latter observation seems to be the common case (Cai et al., 2009; Cvejic et al., 2010), with only a few exceptions.

The weak negative correlation between piceid and resveratrol concentrations in the total data of Goldberg et al. (1996c) may partly be explained. The low values for both piceid and resveratrol in Californian wines are consistent with the observation that, despite high sugar content, some grapes cultivated in this region may fail to express resveratrol synthetic enzymes because of genetic characteristics or to lack of 
environmental stress or response to such a stress, and consequently, little of the free resveratrol is available for glycosylation. Overall, the data argued for the piceid concentration being dependent on the grape cultivar used for the wine production. In addition, the high piceid and very low resveratrol concentrations of Vintage Ports highlight enological techniques (particularly duration of fermentation) as important modulating factors.

The study of Goldberg et al. (1996b) mentioned above measured cis- and transresveratrol content of 761 commercial red wines from 15 countries and regions. The highest concentrations of both isomers were present in wines produced in areas of harsher climate. Cis-resveratrol concentrations of wine seem to be more sensitive than those of trans-resveratrol to unfavourable climatic conditions, with fungal pressure as the most likely mechanism. Wines from Cabernet Sauvignon and Merlot demonstrated a wide range of concentrations (sixfold) that were highly region dependent, whereas those from Pinot Noir and Shiraz showed less variation between and among regions (3.5- for Pinot Noir and 2.5-fold for Shiraz). The investigators concluded that the relationship between the two resveratrol isomers in commercial wines is not a consequence of isomerisation alone and is unlikely to be due to common responses to UV irradiation and fungal attack. Rather, it is due to the extent of conversion of piceids present in the skins to free isomers during fermentation. Another observation made was that wines from the Cabernet Sauvignon grape are the most susceptible wines (of those studied) to regional variation in cis-resveratrol concentration, being high in those from Canada and the Médoc region of Bordeaux and, with one exception, low in wines from warmer climates. Because the ratio of cis:trans-resveratrol was unrelated to climate in this study, the observed differences in the parameter for wines from this cultivar between regions are best explained by oenological factors. It was shown that barrel ageing and the use of certain fining agents reduce trans-resveratrol concentrations more than it does the cis isomer. It was also shown that the formation of trans-resveratrol in the must during fermentation is more rapid than that of cis-resveratrol in several cultivars, emphasising the importance of length of maceration upon their relative concentrations, as described by Jeandet et al. (1995a) for Pinot Noir.

As mentioned earlier in the stilbene section, these compounds are phytoalexins induced by various agents. The presence of such agents in the vineyards as part of the environment is therefore likely to have a major influence on the amount of phytoalexins produced in the developing grapes. Other environmental factors that have a bearing on the stilbene content of wine include locality/soil/altitude where the grapes are grown (Siemann \& Creasy, 1992; Fregoni et al., 1994; Abril et al., 2005; Andrés-de Prado et al., 2007), year of cultivation/production (Pour Nikfardjam et al., 2006b; Eder et al., 2001; Avar et a., 2007), and climatic conditions (Goldberg et al., 1995a; Ribeiro de Lima et al., 1999). When Stervbo et al. (2007) compared the average trans-resveratrol content with the latitude of the producing region, it appeared that the further north on the northern hemisphere, the higher average level of transresveratrol. The trend for the southern hemisphere was that the closer to the equator, the higher average level of trans-resveratrol. Besides the environment, the genetic 
constitution of the grapevine (Vitis vinifera) from which the wine was produced (Siemann \& Creasy, 1992; Ribeiro de Lima et al., 1999; Threlfall et al., 1999; Pour Nikfardjam et al., 2006b; Romero-Perez et al., 1996a; Burns et al., 2001; Adrian et al., 2000a; Eder et al., 2001; Roggero \& Archier, 1994; Loizzo et al., 2011; Pérez-Trujillo et al., 2011; Atanackovic et al., 2012; Baptista et al., 2001) and the conditions during wine making are important. Pinot Noir has been reported to be particularly rich in the commonly analysed stilbenes (McMurtrey et al., 1994, Lamuela-Raventos et al., 1995, Burns et al., 2000, Sato et al., 1997, Eder et al., 2001; Goldberg et al., 1995c).

Wine is also produced from other Vitis species than $V$. vinifera. Lamikanra et al. (1996) reported that the concentrations of resveratrols in wines from Vitis rotundifolia (muscadine) grapes are higher than the resveratrol concentrations in wines from Vitis vinifera and Vitis labruscana grape varieties.

As always, when there is a lot of information available, investigators reach contradictory conclusions. Thus, some have claimed a minor role of winegrape genotype (Gambuti et al., 2004), environment (vintage: Pérez-Trujillo et al., 2011; climate: Eder et al., 2001), and winemaking technology (Atanackovic et al., 2012) on the stilbene content of ready to drink wine; however, these investigators are clearly in the minority.

The wine processing technique has been summarised (Roldán et al., 2003). In traditional winemaking, the process of maceration is initiated when the grape skin is broken and the entire material is exposed to elevated temperatures, encouraging extraction of phenolic compounds (anthocyanins, tannins, and flavours) from the skin (and possibly stems and seeds). During fermentation, the increased temperatures and increasing levels of alcohol encourage the process, with alcohol acting as a solvent, assisting breakdown of organic matter within the grape materials. Maceration continues during the fermentation and can last well past the point when the yeast has converted the sugars into alcohol. When the alcohol content reaches about $10 \%$, the process begins to slow down.

The initial grape juice relieved from the broken grapes is clear-greyish in colour. When white wines are produced, maceration is avoided, or allowed to a limited extent (very short skin contact with the juice before pressing). For rosé wines, red wine grapes are allowed some maceration between the skins and must, and for red wines this is extended, allowing more anthocyanins and resveratrols to be extracted.

As carbon dioxide $\left(\mathrm{CO}_{2}\right)$ is released during the conversion of sugars into alcohol, the gas goes to the top of the must mixture, pushing grape skins and other materials to the top of the maceration vessel. This material forms a cap on the top of the fermentation vessel and may limit the amount of must coming into contact with the skins and reduce extraction efficiency. This is counteracted by pushing down the cap (either with equipment or the traditional method of wine makers of treading with their feet) or by pumping wine out from under (and over) onto the cap.

A few of the studies that focused on elucidating the role of oenological practices on resveratrol content of wine are mentioned below. The processes of viticulture and vinification, which vary between countries, regions, and winemakers, have a very strong influence on the content and profile of stilbenes and other phenolic 
compounds in wine. Jeandet et al. (1995a) compared the effect of classical winemaking of white and red wine on the resveratrol content of the wines. The resveratrol content of white wines that had been macerated $(1.11 \mathrm{mg} / \mathrm{L})$ were tenfold higher than the content in wines just pressed lightly and spent no time on the grape skins ( $0.12 \mathrm{mg} / \mathrm{L})$. Similarly, red macerated wines contained much higher levels of resveratrols $(3.04 \mathrm{mg} / \mathrm{L}$ ) than non-macerated wines ( $0.23 \mathrm{mg} / \mathrm{L}$ ). Because resveratrol is mainly found in the skin and is absent or low in the fruit flesh (Creasy and Coffee, 1988; Jeandet et al., 1991, 1995C), the extraction of resveratrol will be better in wines spending longer time in fermentation in contact with grape skins than in wines not fermented on the skins before pressing. Maceration was shown to increase both transand cis-resveratrol levels in the wine.

Sun et al. (2003) compared the effects of the most important winemaking technologies [carbonic maceration (fermentation of grape juice while still in the undamaged grape), skin fermentation with stem, and skin fermentation without stem] on the resveratrol content in wines produced from the grapevine (Vitis vinifera) variety Castelac. The resveratrol concentrations in wines made by traditional winemaking technologies (fermentation with stems and fermentation without stems) were significantly higher than those in wines made by carbonic maceration. The latter observation has been confirmed by others (Vrhovsek et al., 1997; Clare et al., 2004). However, there was no significant difference in resveratrol concentrations between stem-contact wines and non-stem-contact wine, suggesting that the stems contribute non-important quantities of resveratrol to wines. In addition, prolonged maceration after alcoholic fermentation did not affect the resveratrol concentrations in wines, suggesting that the extraction of resveratrol was complete during or at the end of alcoholic fermentation.

Studying the production of Merlot wines, Poussier et al. (2003), on the other hand, observed differences in stilbene extraction profiles only when cold maceration was applied before alcoholic fermentation.

The time course in stilbene extraction may vary with grape variety and the vinification technology used, mainly because it will take different lengths of time to reach maximum alcoholic content when these factors are altered, something that is likely to contribute to the efficiency of stilbene extraction (Soleas et al., 1995b; Pezet \& Cuenat, 1996; Gambuti et al., 2004; Kostadinovic et al., 2012). After an integrated malolactic fermentation performed by lactic acid bacteria in red wine production (in parallel or following the traditional fermentation) in the study of Pezet and Cuenat (1996), the amount of resveratrol was about twice that measured at the end of alcoholic fermentation, indicating that a pool of resveratrol exists, probably in the form of glucosides (piceids) and/or oligomers (viniferins), from which free resveratrol could be liberated by enzymatic activity of malolactic bacteria. However, FernándezMarin et al. (2014) reported that malolactic fermentation had no effect on the level of trans-resveratrol content in red wines. Vrhovsek et al. (1997) studied the effects of various vinification techniques on the time course for extraction of trans- and cisresveratrol and trans- and cis-piceid into the wine. At the beginning of the fermentation, significant levels of piceides were present (more of cis-piceid than trans- 
piceid), whereas the amount of both aglyconic forms were very low (cis-resveratrol was absent). During the first three days of the fermentation, the concentration of all four forms of resveratrol increased. Subsequently, the glucosidic forms decreased and the aglycone forms increased. Similar data have been published by Mattivi et al. (1995), Clare et al. (2005), and Roggero (1996). Roggero (1996) pointed out that the time course of the stilbene diffusion is very dependent on grape variety. Two other possibilities to explain this trend could be the activity of yeast $\beta$-glucosidases and the increasing alcohol content.

Influence of yeast

Because yeasts have a central role in both the vinification process and the fermentation of grape must (with or without grape skin), it is plausible that yeast metabolism may influence the composition of wines more than it would alcohol content.

Vrhovsek et al. (1997) studied the influence of two yeast strains used in various vinification techniques on the concentration of trans- and cis-resveratrol and resveratrol glucoside isomers in wine. The yeast with higher $\beta$-glucosidase activity significantly decreased the concentration of trans-piceid and increased the concentration of trans- and cis-resveratrol. Other investigators have reported that some yeast strains are able to reduce the resveratrol content of wine (Vacca et al., 1997). Franco et al. (2002) investigated the influence of 14 Saccharomyces cerevisiae strains on the stilbene profile in fermented must of the Cannonau grape. Levels of both trans- and cis-piceid were reduced by all yeast strains, whereas it depended on the yeast strain whether only one or both trans- and cis-resveratrol were increased. It has also been reported that in most cases using French yeast instead of Macedonian yeast in wine production resulted in wines with higher trans-resveratrol and transpiceid levels (Kostadinovic et al., 2012).

Italian Aglianico wines produced with longer maceration times (18 days) contained lower amounts of trans-resveratrol than wines produced with shorter ( 8 days) maceration times. Many investigators have reported an initial positive correlation between time contact with skins and trans-resveratrol extraction (Jeandet et al., 1995; Pezet \& Cuenat, 1996) but a decrease of trans-resveratrol content in must after at least 8 days of maceration (Mattivi et al., 1995; Soleas et al., 1995b; Poussier et al., 2003; Gambuti et al., 2007). The cause for this decrease is not clear. Wines, such as port wine, in which fermentation is prematurely stopped by the addition of ethanol to maintain high sugar concentrations and whose content of resveratrol isomers are very low contain significant concentrations of the piceid-isomers (Baptista et al., 2001). This observation suggests that there is a reciprocal relationship between glycoside concentrations and the free isomers, if the later are at least in part the result of a hydrolysis of piceids and resveratrol oligomers during the fermentation process.

Mattivi and Nicolini (1993) reported that free run wines are richer in resveratrol than the press wines. Free run wines are produced from the juice of grapes spontaneously extracted when grape cell walls are broken down by the weight of the grape berries as they are loaded on top of each other in the press during maceration and fermentation. Four- to eight-day-long carbonic maceration phases, followed by 
vinification without skin contact, give lower resveratrol contents than traditional vinifications for red wines. White wines produced with hyperoxidated musts result in lower contents than normally produced white wines.

In an Australian study using the Cabernet Sauvignon grape, Clare et al. (2004) investigated the effect of various maceration methods on the concentration of transand cis-resveratrol and resveratrol glucoside isomers in grape must during alcoholic fermentation of the wine. Pre-fermentation cold-soaking significantly increased transand cis-resveratrol in free-run, press and mix wine without influencing trans-piceid concentrations and only slightly reduced the extraction of cis-piceid. Free resveratrol was absent in the must heated prior to fermentation, whereas the level of piceids was significantly increased relative to control wine.

Studies of wines from the Canary Islands demonstrate that the extent of piceid hydrolysis differed between wines, with Tantilla wines showing particularly high conversion to the non-glucosylated forms. It was concluded that as trans-resveratrol only accounts for $16-31 \%$ of total stilbenes, it is not enough to characterise a wine by only measuring the trans-resveratrol content. The level of trans-resveratrol and transpiceid on the single-cultivar wines varied between 4.7 and $6.5 \mathrm{mg} / \mathrm{L}$ and 1.6 and $7.3 \mathrm{mg} / \mathrm{L}$, respectively.

\section{Influence of enzymes}

Pectolytic enzymes are added during juice and wine processing to break down pectin in cell walls of grapes and increase juice yield, facilitate colour extraction and stability, and facilitate clarification. Commercial enzymes are typically crude fungal preparations containing impurities such as extraneous enzymes (e.g., $\beta$-glucosidases and esterases). Such impurities might influence certain parameters in wine, such as the ratio between glycosidic and aglyconic forms of stilbenes. Wightman et al. (1997) studied the influence of four preparations of processing enzymes on phenolics in wines produced from the Pinot noir and Cabernet Sauvignon cultivars. The content of trans-resveratrol increased in all enzyme-treated wines and those that had the greatest resveratrol content also contained the greatest quantity of quercetin aglycone.

Before a wine is bottled, it is clarified and stabilised. In these processes, insoluble matter suspended in the wine, such as dead yeast cells, pieces of grape skin, and chemical precipitates, are removed before bottling. Clarification and stabilisation may involve several steps that include fining, filtration, centrifugation, floating, refrigeration, pasteurisation and/or barrel maturation, and racking.

The influence of technological pre-fermentative and fining practices on the quantity of resveratrol in wine have been studied by several investigators. The transand cis-resveratrol contents in a red wine obtained using must hyperoxidation were reduced to about $50 \%$ of the levels in control wines (Castellari et al., 1998). There are contradictory reports on the influence of supplying $\mathrm{SO}_{2}$ to wine. According to Kostadinovic et al. (2012), supplying $\mathrm{SO}_{2}$ during maceration influences stilbene extraction into the red wine only if the $\mathrm{SO}_{2}$ is present at the end of the maceration. However, Castellari et al. (1998) observed that sparging red wines with $\mathrm{SO}_{2}$ and asborbic acid before crushing them resulted in elevated trans-resveratrol 
concentrations compared with control wines. Castellari et al. (1998) claimed that $\mathrm{SO}_{2}$ inactivates grape enzymes and thus protects the polyphenols from oxidation and precipitation during the fermentation process. However, they noted that $30 \mathrm{ppm} \mathrm{SO}_{2}$ was more efficient than $70 \mathrm{ppm}$.

Charcoal, polyvinylpolypyrrolidone (PVPP), egg white (albumin), silica, bentonite, casein, gelatine, and the inert materials cellulose, diatomaceous earth (kieselgur), and filter pad are used as fining agents at the end of wine production to remove haze and alter or remove odour, colour, or taste. They also improve the stability of the wine. Of these, PVPP, charcoal, and casein resulted in more efficient removal of trans- and cisresveratrol (Castellari et al., 1998; Threlfall et al., 1999), and a $0.75 \mu \mathrm{m}$ filter pad improved the removal of trans- but not cis-resveratrol in wines (Soleas et al., 1995b). However, addition of carbon was efficient only in some types of wine. Possibly this was concentration dependent, as Threlfall and co-workers (1999) have shown that low levels of PVPP did not cause significant loss of resveratrol. The other fining agents gave a limited decrease (2-5\%) of trans- and cis-resveratrol, if any (Soleas et al., 1995b; Castellari et al., 1998). Vrhovsek et al. (1997) noted that gelatine also had a marginal influence on piceid content. After charcoal and PVPP treatments, significant correlations were observed between the percentage of residual resveratrol and the total phenols in the wines (Castellari et al., 1998). According to Vrhovsek et al. (1997), the resveratrols showed significant binding to PVPP (up to 90\%), whereas their glycosides showed less binding (up to $70 \%$ ). Viňas et al. (2008) concluded that nonclarified wine contain higher levels of the resveratrol isomers than clarified wine, which contain higher levels than decanted wines.

Influence of microfungi attacks on the growing grapes

Knowing that stilbenes are phytoalexins and contribute to plant defence, the observation that various types of grapevine are more or less susceptible to pathogenic attack of microfungi imply the potential for a difference in stilbene profile and content (Jeandet et al., 1995C).

Perrone et al. (2007) analysed 112 samples of retail red wines from northern $(n=17)$, central $(n=46)$, and southern $(n=49)$ Italy for ochratoxin $A$ as well as for resveratrol-related stilbenes. Ochratoxin $A$ is a nephrotoxic, hepatotoxic, and carcinogenic compound produced by Aspergillus and Penicillium species under specific conditions. These microfungi can be found on grapes and are likely to be inducers of stilbene biosynthesis. Ochratoxin A was detected in $70 \%$ (mean level $0.12 \mu \mathrm{g} / \mathrm{L}$ ) of wines from northern Italy, 59\% (mean level $0.07 \mu \mathrm{g} / \mathrm{L}$ ) from central Italy, and 100\% $(1.36 \mu \mathrm{g} / \mathrm{L})$ from southern Italy. The highest values of ochratoxin A were recorded in Negroamaro- and Primitivo-based wine samples from southern Italy, showing also the highest content of stilbenes. In wines from southern Italy, a positive correlation was observed between the ochratoxin A level and total stilbenes $(r=0.74)$, total resveratrols $(r=0.50)$, or total piceids $(r=0.74)$.

Analysing wines produced from botrytised grapes (attacked by Botrytis cinerea), Pour Nakfardjam et al. (2006a) concluded that the small difference in stilbene content between Hungarian and German (higher in Hungarian) sweet wines is most likely due 
to these wines being produced with different techniques. When Hungarian wines are produced, a longer contact between the alcoholic base (puttony) with the Aszu berries leads to a better extraction of the stilbenes than during German winemaking, which mixes botrytised and healthy grapes from the beginning (except for production of "trockenbeerenauslese", which is only botrytised grapes). The level of stilbenes in botrytised German wines was lower than in German white standard quality wines (Pour Nikfardjam, 2002). This difference was thought to be due to the influence of Botrytis enzymes. Similarly, studying ninety-two samples of Hungarian wines, Avar et al. (2007) noted that the resveratrol content was strongly dependent on vintage, and that high levels of the compound were found in years with little to no fungus pressure in the vineyards. Roldán et al. (2003) showed that high Botrytis cinerea pressure leads to destruction of resveratrol through the stilbene oxidase of the fungus.

Ageing in oak barrels and treatment with charcoal caused major losses of both isomers, but treatment with a $0.75 \mu \mathrm{M}$ filter pad halved the trans-resveratrol concentrations without affecting that of cis-resveratrol. Bentonite, gelatine, silica, egg white, and diatomaceous earth had no effect on either isomer (Soleas et al., 1995b). In agreement with this observation, Gambuti et al. (2007) observed that wines aged six months in oak barrels instead of in stainless steel tanks before bottling contained lower amounts of trans-resveratrol (1.62 vs. $2.50 \mathrm{mg} / \mathrm{L}$ ).

Very few studies have compared the trans-resveratrol levels in red, rosé, and white wines with the levels in their corresponding dealcoholised wines. BelisarioSánchez et al. (2009) observed only small and irrelevant differences between the standard and the de-alcoholised wines studied by these investigators.

Influence of UV-exposure of grapes on the stilbene levels in wine

Because it is possible to induce the production of phytoalexins in grapes and therefore influence the contents in wine produced from these grapes, a number of investigators have studied the possibility to increase the resveratrol content of wines by exposing grapes to abiotic stress after harvest.

UV irradiation of grape clusters increased the trans-resveratrol level in Cynthiana and Noble wine produced in 1994, but did not yield a consistent result in experiments performed for the 1995 and 1996 wines (Threlfall et al., 1999). Cantos et al. (2003a, 2003b) followed the stilbene content of grapes to the content in red wine produced from a variety of wine grapes using a classical maceration procedure, comparing results with untreated grapes with those of UV-C-irradiated grapes. The main stilbenes found in the solid phase (skins) just after grape crushing were transresveratrol, trans-piceatannol, and viniferins (resveratrol polymers). Trace amounts of astringin (piceaannol glucoside) and piceid (resveratrol glucoside) were also detected, possibly partly due to non-optimal extraction procedure (ether). During fermentation, the extraction of stilbenes from the skin increased with increased alcohol content. Highest levels of stilbenes were found on day 4 and 5 after the start of fermentation. There was a substantial (up to tenfold) decrease in resveratrol content from maximum levels to the levels occurring in the final product, as previously demonstrated in normal wine production. It was concluded that the screening of stilbene induction 
capacity in red wine grapes treated with UV-C irradiation could be useful for selecting the most suitable variety to produce stilbene-enriched red wines.

Wine achieved by the combined treatment of the grapes with methyl jasmonate before harvest and UV light after harvest contained a significantly higher (2-5-fold) stilbene concentration (trans-resveratrol, piceatannol, isorhapontigenin, and $\varepsilon$-viniferin) than wine produced from untreated grapes (Fernández-Marin et al., 2014). Of stilbenes in the control wine $(1.25 \mathrm{mg} / \mathrm{L})$, trans-resveratrol made up $92 \%, \varepsilon$ viniferin $7.7 \%$, and the rest were traces of piceatannol. In the wine prepared from methyl jasmonate/UV-treated grapes total, stilbenes were increased ( $4.19 \mathrm{mg} / \mathrm{L}): 70 \%$ were trans-resveratrol, 26\% piceatannol, and $4 \% \varepsilon$-viniferin. Adrian et al. (2001) reported low levels of viniferins in red and rosé wines but no viniferins in white wines. Isorhapontigenin could not be detected in wine but was found at reasonable amounts in pomace (from 0.55 to $1.28 \mathrm{mg} / \mathrm{kg} \mathrm{fw}$ ) (Fernández-Marin et al., 2014).

\section{Influence of storage of wine}

Jeandet et al. (1995b) investigated the influence of ageing on the resveratrol content of wine by analysis of wines of different ages. Red Bourgognes of the variety Pinot noir from the years 1980 to 1992 was studied. Resveratrol was reported to be fairly stable in wine. When the level of resveratrol in the wine was correlated with the fungal disease pressure in the vineyard for a given vintage, it was indicated that conditions leading to infection by Botrytis cinerea enhance resveratrol production, but that extensive grey mould development may destroy the induced phytoalexin. Thus, years with the mould present but resulting in low levels of disease are associated with maximum wine concentration of resveratrol. The investigators interpreted the finding to mean that resveratrol formed in heavy Botrytis-infected grapes might have undergone degradation by exocellular enzymes of Botrytis (e.g., laccase-like stilbene oxidases). Accordingly, this enzyme, which is also active in the musts, could lower the resveratrol content of the wines obtained.

Monagas et al. (2005) followed the stilbene content in red Spanish wines produced from Vitis vinifera varieties Tempranillo, Graciano, and Cabernet Sauvignon during 26 months of ageing in the bottle. The evolution of cis- and trans-resveratrol and cis- and trans-piceid during ageing in the bottle did not follow specific trends, although the trans- form seemed to decrease during the first months of ageing. Levels differed between wines of various cultivars but were not related to storage time in this study. Other investigators have found indications of lower resveratrol contents in aged wines (Adrian et al., 200ob; Careri et al., 2003; Abril et al., 2005). For instance, Mattivi and Nicolini (1993) noted that moderately high temperatures during storage can cause a decrease of resveratrol in wines that are low in $\mathrm{SO}_{2}$ and have a high pH. In contrast to resveratrol, piceid was not reduced in aged wines (Adrian et al., 200ob).

Careri et al. (2004) studied the degradation of trans-resveratrol in one type of red wine during a one-month period after opening a bottle. The trans-resveratrol level decreased by $74 \%$ and cis-resveratrol by $28 \%$. The investigators concluded that these data probably indicate that the trans-isomer rapidly converts to the cis-isomer and subsequently both isomers polymerise to form resveratrol oligomers. Similar studies have been performed by 
Czech scientists (Kolouchová-Hanzliková et al., 2004). They analysed Czech wines for stilbenes directly after being opened, as well as 2 and 17 days later, the latter two time points after resealing the bottle and storing it at room temperature in diffused daylight. The ratio of trans-/cis-resveratrol was significantly reduced during the storage from an initial value of 1.0-4.75 depending on wine and vintage.

In conclusion, trans- and cis-resveratrol and trans- and cis-piceid levels in wine differ between products and are influenced by numerous factors. Wine produced from stressed grapes may contain increased amounts of these constituents as well as polymers of resveratrol (viniferins). Red wines usually contain higher amounts of stilbenes than rosé wines that contain higher amounts than white wines. Levels of these stilbenes are summarised in Table 38. There are only a few studies available that have studied other stilbenes.

The first reports that wine contains trans-astringin (the $3-O-\beta$-D-gluco(pyrano)side of $3^{\prime}$-hydroxy-resveratrol) appeared in 1999 (Carando et al., 1999; Ribeiro de Lima et al., 1999), but the two research teams reported quite different levels. Carando and coworkers analysed poorly defined wines from the South of France and detected levels from $0.09 \mathrm{mg} / \mathrm{L}$ to $0.29 \mathrm{mg} / \mathrm{L}$. De Lima et al. (1999) reported much higher concentrations and that no less than about $53 \%$ of the 46 studied Portuguese red wines contained the compound ( $9-35 \mathrm{mg} / \mathrm{L}$ ), whereas $26 \%$ of the 74 Portuguese white wines contained trans-astringin (9-15.6 mg/L). An even higher trans-astringin concentration $(80 \mathrm{mg} / \mathrm{L})$ was found in a young red press wine produced conventionally from grapes of the Syrah variety. A few years later, Vitrac et al. (2002) published data on 49 commercial wines from AOC Bergerac, France, supporting the higher values already reported. Some of the analysed wines contained more than $30 \mathrm{mg} / \mathrm{L}$ (Vitrac et al., 2002). However, there was a wide variation in content between the wine samples and the compound was not detected in all of them.

Vitrac et al. (2002) was also the first to report on the occurrence of pallidol (a resveratrol dimer) in red wines. No pallidol was found in white wines. The concentrations in red wines were between 0.5 and $4.8 \mathrm{mg} / \mathrm{L}$ (mean $2.5 \mathrm{mg} / \mathrm{L}$ ). It has been suggested that pallidol is a fungal metabolite produced by oxidation of resveratrol (Cichewicz et al., 2000).

\subsubsection{Stilbenes in legumes}

In addition to occurring in the already mentioned foods, resveratrol (and other stilbenes) has been identified in the legume mung bean Vigna radiata. However, it was not quantified (Kim et al., 2013.)

\subsubsection{Stilbenes in solanaceous food plants}

In parallel with reporting on the occurrence of resveratrol and piceid preferentially in the skin of grape berries, Ragab et al. (2006) reported resveratrol to occur in the skin of tomato (Lycopersicon esculentum) fruit but not in the flesh. However, levels were only about $1 \%$ of the resveratrol content of grape berries. Total resveratrol content 
accumulated slowly after the breaker stage (2.5 weeks, fruit becoming orange) and increased slightly after 3.5 weeks to a maximum at 4 weeks. At this stage of maturity, the fruit skin contained $18.4 \pm 1.6 \mathrm{mg} / \mathrm{kg} \mathrm{dw}$. Thereafter, resveratrol accumulation abruptly ceased at 4.5 weeks. Trans-Resveratrol was identified in all but one of the five tomato varieties studied but the level varied by several orders of magnitude between varieties (n.d. $-15.3 \mathrm{mg} / \mathrm{kg}$ ). Cis-resveratrol was only detected in two of the tomato varieties (n.d. $-2.71 \mathrm{mg} / \mathrm{kg}$ ) and trans- and cis-piceid in only one of the varieties (n.d.0.10 and n.d. $-0.26 \mathrm{mg} / \mathrm{kg}$, respectively). The tomato cultivar MicroTom contained a much higher stilbene content than the other tomato cultivars.

Canadian investigators analysed a tomato variety with a deviating form (grape tomato) for stilbenes and detected about $0.170 \mathrm{mg} / \mathrm{kg}$ trans-resveratrol but no cisresveratrol and trans- and cis-piceid (Huang and Mazza, 2011). The same investigators found no stilbenes in red and orange bell pepper. In green bell pepper, they observed non-quantifiable traces of cis-piceid.

\subsubsection{Stilbenes in mulberry}

It has been known since the middle of the 1970 s that heartwood of several Morus species, including the Mulberry (M. alba), contain several stilbenes (Deshpande et al., 1975). For example, M. alba was shown to contain resveratrol, oxyresveratrol, and dihydrooxyresveratrol, but not piceatannol, 3,4'-dihydroxydihydrostilbene and 3,2', $4^{\prime}$ trihydroxydihydrostilbene. Much later, Song et al. (2009) studied 38 cultivars of seven Morus species as well as one hybrid between two species in relation to the phytochemical profile of their fruits, fruit juices, and leaves. Whereas fruit juices contained neither resveratrol nor oxyresveratrol, these compounds could be identified in fruit solids remaining after pressing out the juice. Oxyresveratrol was identified in all 41 fruit samples analysed and resveratrol in 13 of the samples. The level of oxyresveratrol in the 12 samples of $M$. alba was between non-detectable levels and $36.3 \mathrm{mg} / \mathrm{kg}$ and the level of resveratrol between non-detectable levels and $4.8 \mathrm{mg} / \mathrm{kg}$. Varieties from the mulberry species Morus atropurpurea contained the highest levels of these stilbenes.

A few years later, Choi et al. (2013) reported three stilbene derivatives in mulberry. These are trans-resveratrol, oxyresveratrol $\left(2,4,3^{\prime}, 5^{\prime}\right.$-tetrahydroxystilbene), and oxyresveratrol $3^{\prime}-O-\beta-D-g l u c o s i d e$. By studying different mulberry cultivars and growing some at different localities, it became clear that both genoype and environment influenced stilbene levels. Thus, the oxyresveratrol 3'-O-beta-Dglucoside content varied between 237 and $1967 \mathrm{mg} / \mathrm{kg} \mathrm{dw}$, whereas oxyresveratrol and trans-resveratrol occurred at non-detectable or very low levels (trans-resveratrol below $11 \mathrm{mg} / \mathrm{kg} \mathrm{dw}$ ) (Choi et al., 2013). When mulberry produced from the site resulting in the highest total level of stilbenes was processed by roasting for five minutes, no marked effect on the level of the studied compounds was observed. Microwaving the mulberry berries for five minutes reduced the level of the various stilbenes by $10-30 \%$. Steaming berries for thirty minutes had a more pronounced effect, resulting in a reduction of oxyresveratrol 3 '-O- $\beta$-D-glucoside content to about 
$30 \%$ of the control level, oxyresveratrol to $90 \%$ of the control level, and transresveratrol not at all. It was hypothesised that oxyresveratrol 3'-O-beta-D-glucoside in the mulberry is partly converted into its corresponding aglycone oxyresveratrol by enzymatic conversion during the drying and ageing processes (Choi et al., 2013).

\subsubsection{Stilbenes in sugar cane}

Juice of cut sugar cane has been analysed for trans-resveratrol and piceatannol. Juice prepared directly after cutting the sugar cane contained undetectable levels of these stilbenes. The compounds were detectable in juice prepared three days later and reached optimal amounts after seven days when it contained $1.2 \mathrm{mg} / \mathrm{L}$ and $8.5 \mathrm{mg} / \mathrm{l}$ piceatannol (Boue et al., 2013). Sugar prepared from sugar cane has not been analysed for stilbenes.

\subsubsection{Stilbenes in rhubarb}

Already in 1980, Rupprich and co-workers noted that rhaponticin (3,5,3'-trihydroxy$4^{\prime}$-methoxystilbene $3-0$ - $\beta$-glucoside) is synthesised in the rhubarb rhizome. Further insights into this issue was given by Püssa et al. (2009) who investigated the roots and the edible leaf stalks (the petioles) of the garden rhubarb (Rheum rhaponticum L.) for the occurrence of polyphenolic compounds. This study aimed to identify compounds rather than quantify them. The roots of $R$. rhaponticum were very rich in various hydroxystilbenes and contained four main substance groups, being derivatives of trans-piceatannol, trans-resveratrol, trans-rhapontigenin, and transdeoxyrhapontigenin. Additionally, pterostilbene acetylglucosides were identified in the root samples. The stilbene profile of the petioles was similar to that of the roots but the content of individual substances was remarkably lower.

\subsubsection{Other products containing stilbenes}

Trans-resveratrol was first isolated from the roots of the Asian plant Veratrum grandiflorum (Takaoka, 1940) and has since then been found in the bark, leaves, and heartwood of several trees as well as in a number of plants, sometimes in combination with other stilbenes. It is usually not known if the analysed samples have been healthy or had been stressed.

Resveratrol has also been identified in many medicinal plants, such as Calligonum leucocladum (Okasaka et al., 2004), Cassia quinquangulata, C. garrettiana (Hata et al., 1979; Pezzuto, 2011), Erythropleum lasiantum (Orsini et al., 1997), Ficus barteri fruits (Ogungbamila et al., 1997), Reynoutria japonica (Kimura et al., 1995), Polygonum cuspidatum (Jayatilake et al., 1993; Vastano et al., 2000), and Smilax glabra (Chen et al., 1999). Paul et al. (1999) demonstrated that darakshasava, an ayurvedic medicine from India, which main ingredient is Vitis vinifera, contains resveratrol and pterostilbene. 
Bees collect a resinous substance called propolis for coating hive parts and the cell interiors of the honeycomb and also to seal cracks and crevices in the hive. Ghisalberti et al. (1978) demonstrated that pterostilbene is a major constituent of propolis.

Finally, there is a report that olives are void of stilbenes (Counet et al., 2006).

\subsection{Coumestan levels in food}

Coumestans, with coumestrol and 4'-O-methylcoumestrol as the most important individual compounds, exhibit a close structural similarity to isoflavones. The structural formula of coumestrol is shown in Figure $1 \mathrm{D}$. Coumestrol is derived from daidzein by a series of reactions that include hydroxylation/oxidation at the $\mathrm{C}_{2}$ position and ring closure with loss of water between the hydroxyl on $\mathrm{C}_{4}$ of the heterocycle (isoflavone numbering) and a hydroxyl on $\mathrm{C}^{\prime}$ ' of the B-ring (Dixon, 2004).

Coumestrol has been recognised as a phytoestrogen owing to its high affinity for ERs, its affinity being approximately 30-100 times stronger than that of the soybean isoflavonoids. It exhibits antagonist functions in humans by competing with endogenous oestrogens for ERs (Bacaloni et al., 2005).

In foodstuffs, coumestans are mainly found in legumes such as alfalfa, clover, and soybean sprouts (Cornwell et al., 2004). Beggs et al. (1985) studied the induction of coumestrol by UV radiation in leaves of the common bean (Phaseolus vulgaris $\mathrm{L}$. var Saza) using the compound as a marker for DNA damage. No coumestrol was induced unless the wavelength of the inducing light included wavelengths below $300 \mathrm{~nm}$. Its formation could be reversed by treatment with white, but not red light, after the UV irradiation. The formation of coumestrol by UV light was also inhibited by coirradiation with blue light at a high rate. The investigators suggested that coumestrol formation is mediated via UV-induced pyrimidine dimer formation in the plant DNA and that the blue light effect is brought about by induced dimer repair. Thus, the investigators believed the coumestrol level is increased upon plant stress.

In line with this hypothesis, other investigators demonstrated that soybeans infected with fungi increased their levels of coumestrol compared with non-infected soybeans (Bové et al., 2000; Lee et al., 2013). Thus, the coumestans show the typical behaviour of a phytoalexin. Coumestrol is synthesised by the plant when specific stress signals are received. The cotyledon of healthy soybean seeds usually contains negligible levels of coumestrol. In experimental systems, Boué et al. (2000) demonstrated that phytoalexin levels increased significantly the first few days after the cotyledons were exposed to various Aspergillus microfungi (A. sojae, A. oryzae, $A$. niger, and $A$. flavus), reaching a maximum level three days after the inoculation. Although the general trend was the same in different soybean varieties, there were small differences between varieties. Interestingly, several Aspergillus species are used to prepare fermented foods and beverages, such as koji, the starting material for production of soy sauce and miso (Luh, 1995; Liu, 1997).

Having a molecular structure that reminds us of isoflavones, it is not unexpected that the chemical analysis of coumestans uses nearly identical methods to those used 
when analysing total isoflavones. Knuckles et al. (1976) pointed out that coumestrol is more efficiently extracted at acidic conditions than it is at slightly alkaline conditions.

In the sections below, the data identified on the coumestrol content of various foodstuffs are summarised. It can be noted that for time constraints, the literature search for available data on coumestrol levels in food was not as systematic as the searches for the occurrence of isoflavones, lignans, and stilbenes in foods.

\subsubsection{Legumes}

Like isoflavones, coumestans are found mostly in legumes. But it is found in far from all legumes. Thus, no coumestrol, or very low levels ( $\leq 0.02 \mathrm{mg} / \mathrm{kg} \mathrm{fw}$ or $\leq 0.05 \mathrm{mg} / \mathrm{kg}$ $\mathrm{dw}$ ), have been reported for broad bean (Vicia faba), chickpeas (Cicer arietinum), cowpea (Vigna unguiculata), common bean (Phaseouls vulgaris), lentil (Lens culinaris), lupin (Lupinus mutabilis), mung bean (Vigna radiata), peanut (Arachis hypogaea), peas (Pisum sativum), pigeon pea (Cajanus cajan), and Tepary beans (Phaseolus acutifolius) (Franke et al., 1994; Kaufman et al., 1997; Mazur \& Adlercreutz, 1998; Mazur et al., 1998b; Horn-Ross et al., 2000; Liggins et al., 2000b; Thompson et al., 2006; Kuhnle et al., 2007, 2008b; Konar et al., 2012). Small amounts were found in licorice (0.038 $\mathrm{mg} / \mathrm{kg} \mathrm{fw}$ ) and lima beans (n.d.-0.1 mg/kg fw) (Franke et al., 1994; Mazur et al., 1998b; Liggins et al., 2000b; Thompson et al., 2006) and higher amounts in soybeans, but the levels varied dependent on the material under investigation.

Konar et al. (2012) compared the level of coumestrol in six legumes (chickpea, red kidney bean, haricot bean, yellow lentil, red lentil, and green lentil), and in agreement with the data given above, found comparatively low and equal levels. The lowest amount occurred in chickpeas $(0.0065 \mathrm{mg} / \mathrm{kg} \mathrm{fw})$; the other legumes studied contained between 0.0164 and $0.0185 \mathrm{mg}$ coumestrol $/ \mathrm{kg} \mathrm{fw}$.

De Lima and co-workers (2014) investigated the occurrence of coumestrol in 16 non-related genotypes of the common bean (Phaseolus vulgaris), representing seven types of seed coat characteristics and different responses to pathogens and pests. As expected for a phytoalexin, six of the genotypes contained non-detectable levels of coumestrol. The others contained levels from 0.75 to $17.67 \mathrm{mg} / \mathrm{kg} \mathrm{dw}$. Black, carioca, and bolinha beans contained coumestrol, whereas striped and white and red beans did not. It is not possible from these data to determine whether the variation in coumestrol level was due to genetic differences between the varieties or to the different environmental conditions where the materials had grown.

Diaz-Batalla et al. (2006) studied the influence of germination on the level of coumestrol in seeds of seven varieties of the common bean (Phaseolus vulgaris). A considerable difference was found in coumestrol content 72 hours after initiating the germination process (between 2.4 and $35.6 \mathrm{mg} / \mathrm{kg} \mathrm{dw}$ ) compared with the trace levels found in the raw and cooked bean seed flour.

Coumestrol concentrations reported in soybean seeds were between nondetectable levels and $2.25 \mathrm{mg}$ per $\mathrm{kg}$ fw (Zhou et al., 1998; Horn-Ross et al., 2000; Liggins et al., 2000b; Goda et al., 2002; Sakakibara et al., 2003; Primomo et al., 2005; Thompson et al., 2006; Lundry et al., 2008; Xu \& Chang, 2008b; Bermann et al., 2011; 
Lepping et al., 2013). Several soybean products have also been analysed for the same constituent. These studies revealed that soy milk contains up to $2.30 \mathrm{mg}$ coumestrol/kg fw (Wang et al., 1990; Horn-Ross et al., 2000; Thompson et al., 2006; Kuhnle et al., 2008a), soy nuts $0.37 \mathrm{mg} / \mathrm{kg}$ fw (Thompson et al., 2006), and soy protein powder, soy sauce, textured soy protein, and soya infant formula very low amounts ( $\leq 0.005 \mathrm{mg} / \mathrm{kg} \mathrm{fw}$ ) of coumestrol (Wang et al., 1990; Horn-Ross et al., 2000; Thompson et al., 2006; Kuhnle et al., 2008a; Song et al., 1998).

Romani et al. (2003) investigated different plant parts (roots, stems, leaves, cotyledons, pods, and seeds) of three soybean varieties sampled three times during the growing period for their coumestan contents. Although no coumestrol, coumestrol-7-O-glucoside, and coumestrol-malonylglucoside could be detected in the stem, leaves, and pods in any of the three varieties, roots of the only variety studied contained 876,704 and $2,497 \mathrm{mg} / \mathrm{kg} \mathrm{dw}$ of these constituents.

Alfalfa sprout is a fairly common part of the human diet, and alfalfa forage very common in livestock feed. Considerable amounts of coumestrol has been found not only in alfalfa sprouts $(0.025-1.05 \mathrm{mg} / \mathrm{kg} \mathrm{fw})$, but also in legume sprouts such as mungbean sprouts $(1.37-20.00 \mathrm{mg} / \mathrm{kg} \mathrm{fw})$, clover sprouts $(0.98-281 \mathrm{mg} / \mathrm{kg} \mathrm{fw})$, and soybean sprouts (n.d.-2.36 mg/kg fw) (Knuckles et al., 1976; Franke et al., 1994; Müllner and Sontag, 1999; Horn-Ross et al., 2000; Liggins et al., 2000b; Thompson et al., 2006; Lee et al., 2013). Coumestrol is the major phytoestrogen in alfalfa, but, as demonstrated by Hong et al. (2011), it is far from the only one.

Coumestrol has also been identified in other important feed components. Knuckles et al. (1976) reported that whole alfalfa contains from 11 to $118 \mathrm{mg}$ coumestrol $/ \mathrm{kg} \mathrm{fw}$. The considerable variation was attributed to the phytoalexin characteristics of the compound, where the levels were strongly influenced by abiotic stress (e.g., climatic conditions) and biotic stress induced by insect and disease pests. Fifteen other legume or cereal products used as animal feed or human food contained low amounts (<1 $\mathrm{mg} / \mathrm{kg} \mathrm{dw}$ ) of coumestrol.

Andersen et al. (2008) studied the influence of feeding Lucerne and grass/clover silage to dairy cows on the concentration of phytoestrogens in the milk produced. Milk and pooled feed samples were analysed for the concentration of isoflavones, coumestans, and lignans. The concentration of coumestrol (and secoisolariciresinol) increased with the proportion of the feed being Lucerne, whereas the concentration of isoflavones was similar across the diets. The concentration of coumestrol in the milk was significantly higher for cows fed the Lucerne diet than the grass/clover diet. Thus, milk from cows fed the pure Lucerne silage diet contained $3.2 \mu \mathrm{g} / \mathrm{L}$ coumestrol, whereas milk from cows fed the grass/clover silage diet contained $0.82 \mu \mathrm{g} / \mathrm{L}$ coumestrol. It should be noted that the concentration of phytoestrogens in bovine milk is low compared with food sources rich in those phytoestrogens. 


\subsubsection{Occurrence in other foods than legumes}

A good number of vegetables have been analysed for their content of coumestrol. In most cases, no coumestrol, or only traces or very low levels of the compound, have been found (Liggins et al., 2000b; Horn-Ross et al., 2000; Kuhnle et al., 2007, 2009b; Clarke et al., 2013a). The highest levels reported were in frozen Brussels sprout, but it was only $0.4 \mathrm{mg} / \mathrm{kg} \mathrm{dw}$ (Knuckles et al., 1976). The same conclusion can be drawn about the occurrence of coumestans in fruits, berries, and cereals. The highest amount detected in fruits (grapefruit) was $0.5 \mathrm{mg} / \mathrm{kg}$ fw (Horn-Ross et al., 2000), and the highest amount in cereals was found in black rice Mochi $(0.3 \mathrm{mg} / \mathrm{kg} \mathrm{fw})(C l a r k e ~ e t$ al., 2013a).

Several root vegetables have been analysed for coumestrol, but most of them (beetroot, parsnip, radish, swede) did not contain the compound (Mazur \& Adlercrutz 1998; Thompson et al., 2006; Liggins et al., 2000b; Kuhnle et al. 2007). Traces of the compound were observed in tapioca (up to $0.001 \mathrm{mg} / \mathrm{kg} \mathrm{fw}$; Liggins et al., 2002; Kuhnle et al. 2009a) and low levels in sweet potatoes ( $0.03 \mathrm{mg} / \mathrm{kg} \mathrm{fw}$; Kuhnle et al. 2007), turnips (0.02 mg/li Liggins et al., 2000b), and potatoes (0.0011-0.0058 mg/kg dw; Kuhnle et al., 200gb).

Four oilseed crops have been analysed for coumestrol. Whereas no coumestrol was found in seeds of oilseed rape (Kuhnle et al. 2008b), low levels occurred in sunflower seed ( $0.002 \mathrm{mg} / \mathrm{kg} \mathrm{fw}$ ) and sesame seed (0.004 $\mathrm{mg} / \mathrm{kg} \mathrm{fw})$, and slightly more (0.47 mg/kg fw) in flaxseed (Mazur \& Adlercrutz 1998; Liggins et al., 2000a; Thompson et al., 2006).

Also, nuts contain no or very low levels of coumestrol. The compound could not be identified or not quantified in coconuts, pine nuts, and walnuts (Mazur \& Adlercrutz 1998; Liggins et al., 2000a; Thompson et al., 2006; Kuhnle et al., 2008b; Clarke et al., 2013), and occurred at low levels in cashew nuts (not detectable $-0.004 \mathrm{mg} / \mathrm{kg} \mathrm{fw}$; Thompson et al. 2006; Kuhnle et al., 2008b), chestnuts (0.024 mg/kg fw; Liggins et al., 2000a; Thompson et al. 2006), hazelnuts (traces-0.003 mg/kg fw; Liggins et al., 2000a; Thompson et al. 2006; Kuhnle et al. 2008b), peanuts (0.001-0.02 mg/kg fw; Liggins et al., 2000a; Thompson et al. 2006; Kuhnle et al. 2008b), and pecan nuts (n.d.-0.003 $\mathrm{mg} / \mathrm{kg}$ fw; Thompson et al. 2006; Kuhnle et al. 2008b).

Only a few spices have been analysed for the occurrence of coumestans. Coumestrol was not found in ginger root and arrow root (Kuhnle et al. 2009a; Clarke et al., 2013), whereas $0.62 \mathrm{mg} / \mathrm{kg}$ fw was found in canned chilli (Horn-Ross et al. 2000).

Of eight seafoods analysed for the presence of coumestrol, the compound was detected at very low levels ( $0.001 \mathrm{mg} / \mathrm{kg} \mathrm{fw}$ ) in seaweed and canned tuna (Thompson et al. 2006; Kuhnle et al., 2008a). Fresh tuna contained higher, but not impressive levels ( $0.71 \mathrm{mg} / \mathrm{kg} \mathrm{fw}$ ) of the compound (Horn-Ross et al. 2000). No coumestrol, or only traces of the compound, was observed in cod, salmon, mussels, and prawns (Kuhnle et al. 2008a).

Products of animal origin only contain traces of coumestrol (Horn-Ross et al., 2000; Liggins et al., 2000b; Antignac et al., 2004; Thompson et al., 2006; Hoikkala et al., 2007; Kuhnle et al., 2007, 2008a). 
Clarke et al. (2013) analysed quorn, a protein produced by a microfungus, for coumestrol but was unable to identify it.

Beverages contain very low quantities of coumestans. The highest amounts of coumestrol have been found in fruit juices, with orange juice containing $0.53 \mathrm{mg} / \mathrm{kg} \mathrm{fw}$ and lemon juice $0.13 \mathrm{mg} / \mathrm{kg}$ dw (Horn-Ross et al., 2000; Kuhnle et al., 2007).

Of course, depending on the ingredients when manufacturing a food, some foodstuffs may contain more than trace levels of coumestrol. For example, Chinese black bean sauce has been reported to contain $3.05 \mathrm{mg} / \mathrm{kg} \mathrm{fw}$ and doughnuts 2.43 $\mathrm{mg} / \mathrm{kg} \mathrm{fw}$ (Horn-Ross et al., 2000).

Leuner and co-workers (2012) undertook a study to analyse coumestrol in fortyone neglected tropical and subtropical legumes used as human food or animal feed. The compound was identified in six of the forty-one species. Highest amounts were found in seeds of Melilotus officinalis, which contained $105 \mathrm{mg} / \mathrm{kg} \mathrm{dw}$. The other legumes shown to contain coumestrol were the green parts of Macroptilium atropurpureum (23.3 mg/kg dw), Calopogonium mucunoides (20.2 mg/kg dw), Macroptilium lathyroides $(8.0 \mathrm{mg} / \mathrm{kg} \mathrm{dw})$, and Aeschynomene americana $(4.0 \mathrm{mg} / \mathrm{kg}$ $\mathrm{dw})$, as well as leaves of Andira macrothyrsa ( $8.2 \mathrm{mg} / \mathrm{kg} \mathrm{dw}$ ). 


\section{References}

Abarzua, S., T. Serikawa, M. Szewcyk, D-U. Richter, B. Piechulla \& V. Briese (2012). Antiproliferative activity of lignans against the breast carcinoma cell lines MCF 7 and BT 20. Gynecologic Oncology 285(4):1145-1151. https://doi.org/10.1007/s00404-011-2120-6

Abril, M., A.I. Negueruela, C. Pérez, T. Juan \& G. Estopaňán (2005). Preliminary study of resveratrol content in Aragón red and rosé wines. Food Chemistry 92:729-736. https://doi.org/10.1016/j.foodchem.2004.08.034

Adrian, M., P. Jeandet, J. Veneau, L.A. Weston \& R. Bessis (1997). Biological activity of resveratrol, a stilbenic compound from grapevines, against Botrytis cinerea, the causal agent for gray mold. Journal of Chemical Ecology 23(7):1689-1702. https://doi.org/10.1023/B:JOEC.0000006444.79951.75

Adrian, M., H. Rajaei, P. Jeandet, J. Veneau \& R. Bessis (1998). Resveratrol oxidation in Botrytis cinerea conidia. Phytopathology 88:472-476. https://doi.org/10.1094/PHYTO.1998.88.5.472

Adrian, M., P. Jeandet, A.C. Douillet-Breuil, L. Tesson \& R. Bessis (2000a). Stilbene content of mature Vitis vinifera berries in response to UV-C elicitation. Journal of Agricultural and Food Chemistry 48(12):6103-6105. https://doi.org/10.1021/jfooog910, http://www.ncbi.nlm.nih.gov/pubmed/11312782

Adrian, M., P. Jeandet, A.C. Breuil, D. Levite, S. Debord \& R. Bessis (200ob). Assay of resveratrol and derivative stilbenes in wines by direct injection high performance liquid chromatography. American Journal of Enology and Viticulture 51(1):37-41.

Aehle, E., U. Müller, P.C. Eklund, S.M. Willför, W. Sippl \& B. Dräger (2011). Lignans as food constituents with estrogen and antiestrogen activity. Phytochemistry 72(18):2396-2405. https://doi.org/10.1016/j.phytochem.2011.08.013

Aguamah, G, E, P. Langcake, D.P. Leworthy, J.A. Page, R.J. Pryce \& R.N. Strange (1981). Two novel stilbene phytoalexins from Arachis hypogaea. Phytochemistry 20(6):1381-1383. https://doi.org/10.1016/0031-9422(81)80044-1

Akiyama, T., J. Ishida, S. Nakagawa, H. Ogawara, S.-I. Watanabe, N. Itoh, M. Shibuya \& Y. Fukami (1987). Genistein, a specific inhibitor of tyrosine-specific protein kinases. Journal of Biological Chemistry 262:5592-5595.

Alasalvar, C. \& F. Shahidi (2009) Tree nuts: Composition, phytochemicals and health effects: An overview. In: Tree nuts: Composition, phytochemi-cals and health effects, Cesarettin Alasalvar and Fereidoon Shahidi (Eds.), CRC Press, Boca Raton, pp. 1-10.

Algar, E., Ramos-Solano, B., Garcia-Villaraco, A. Saco Sierra, M.D., Gómez, M.S.M. \& E.J. Gutiérrez-Mañero (2013). Bacterial bioeffectors modify bioactive profile and increase isoflavone content in soybean sprouts (Glycine max var Osumi). Plant foods for human nutrition 68(3):299-305. https://doi.org/10.1007/s11130-013-0373-X

Alonso, A.M., C. Domínguez, D.A. Guillén \& C.G. Barroso (2002). Determination of antioxidant power of red and white wines by a new electrochemical method and its correlation with polyphenolic content. Journal of Agricultural and Food Chemistry 50(11):3112-3115. https://doi.org/10.1021/jfo116101, http://www.ncbi.nlm.nih.gov/pubmed/12009971

Alves, R.C., Almeida, I.M.C., Casal, S. \& M.B.P.P Oliveira (2010). Isoflavone in coffee: influence of species, roast degree, and brewing method. Journal of Agricultural and Food Chemistry 58(5):3002-3007. https://doi.org/10.1021/jf9039205

Andersen, C., Weisberg, M.R., Hansen-Møller, J. \& K. Serjsen (2008). Effect of forage on the content on phyto-oestrogens in bovine milk. Animal 3(4):617-622.

https://doi.org/10.1017/S1751731108003698 
Antignac, J.-P., Cariou, R., Bizec, B.L. \& F. André (2004). New data regarding phytoestrogens content in bovine milk. Food Chemistry $87(2): 275-281$.

https://doi.org/10.1016/j.foodchem.2003.12.013

AOAC (2005) Official Methods of Analysis of AOAC International (2005). AOAC Official Method 2001.10 Determination of isoflavones in soy and selected foods containing soy.

Arce, L., M.T. Tena, A. Rios \& M. Valcárcel (1998). Determination of trans-resveratrol and other polyphenols in wines by a continuous flow sample clean-up system followed by capillary electrophoresis separation. Analytica Chimica Acta 359:27-38. https://doi.org/10.1016/Sooo32670(97)00668-5

Artés-Hernández, F., F. Artés \& F.A. Tomás-Barberán (2003). Quality and enhancement of bioactive phenolics in cv. Napoleon table grapes exposed to different postharvest gaseous treatments. Journal of Agricultural and Food Chemistry 51(18).5290-5295.

https://doi.org/10.1021/jfo30037d, http://www.ncbi.nlm.nih.gov/pubmed/12926872

Atanackovíc, M., A. Petrovíc, S. Jovíc, L. Gojkovíc-Bukarica, M. Bursác \& J. Cvejíc (2012). Influence of winemaking techniques on the resveratrol content, total phenolic content and antioxidant potential of red wines. Food Chemistry 131:513-518.

https://doi.org/10.1016/j.foodchem.2011.09.015

Aussenac, T., Lacombe, S. \& J. Daydé (1998). Quantification of isoflavons by capillary zone electrophoresis in soybean seeds: effects of variety and environment. American Journal of Clinical Nutrition 68(Suppl):1480S-1485S.

Avar, P., M.S. Pour Nikfardjam, S. Kunsági-Máté, G. Montskó, Z. Szabó, K. Bödde, R. Ohmacht \& L. Márk (2007). Investigation of phenolic components of Hungarian wines. International Journal of Molecular Sciences 8:1028-1038. https://doi.org/10.3390/i8101028

Bacaloni, A., Cavaliere, C., Faberi, A., Foglia, P., Samperi, R. \& Laganà, A. (2005). Determination of isoflavones and coumestrol in river water and domestic wastewater savage treatment plants. Analytica Chimica Acta 531(2):229-237. https://doi.org/10.1016/j.aca.2004.10.037

Baderschneider B. \& P. Winterhalter (2000). Isolation and characterization of novel stilbene derivatives from Riesling wine. Journal of Agricultural and Food Chemistry 48:2681-2866. https://doi.org/10.1021/jf991348k

Bais, A.J., P.J. Murphy \& I.B. Dry (2000). The molecular regulation of stilbene phytoalexin biosyntheis in Vitis vinifera during grape berry development. Australian Journal of Plant Physiology 27:425-433. https://doi.org/10.1071/PPooo07_CO

Ballard, T.S., P. Mallikarjunan, K. Zhou \& S.F. O'Keefe (2009). Optimizing the extraction of phenolic antioxidants from peanut skins using response surface methodology. Journal of Agricultural and Food Chemistry 57:3064-3072. https://doi.org/10.1021/jf8030925

Ballistreri, G., E. Arena \& B. Fallico (2009). Influence of ripeness and drying process on the polyphenols and tocopherols of Pistacia vera L. Molecules 14(11):4358-4369.

http://www.ncbi.nlm.nih.gov/pubmed/19924070

Baptista, J.A.B., J.F.da F. Tavares \& R.C.B. Carvalho (2001). Comparison of polyphenols and aroma in red wines from Portuguese mainland versus Azores Islands. Food Research International 34:345-355. https://doi.org/10.1016/Sog63-9969(o0)00174-5

Barkhem, T., B. Carlsson, Y. Nilsson, E. Enmark, J. Gustafsson \& N. Nilsson (1998). Differential response of estrogen receptor alpha and estrogen receptor beta to partial estrogen agonists/antagonists. Molecular Pharmacology 54(1):105-112.

Barnes, S., Kirk, M. \& L. Coward (1994). Isoflavones and their conjugates in soy foods: Extraction conditions and analysis by HPLC-mass spectrometry. Journal of Agricultural and Food Chemistry 42:2466-2474. https://doi.org/10.1021/jfooo47a019

Barnes, S., B. Boersma, R. Patel, M. Kirk, V.M. Darley-Usmar, H. Kim \& J. Xu (2000). Isoflavonoids and chronic disease: mechanism of action. Biofactors 12(1-4):209-215. https://doi.org/10.1002/biof.5520120133

Bavaresco, L. (1993). Action du potassium sur la synthèse induite du stilbène chez différentes variètès de vigne. Le Bulletin de L'OIV No751-752:675-689. 
Bavaresco, L. \& R. Eibach (1987). Investigations on the influence of N fertilizer on resistance to powdery mildew (Oidium tuckeri), Downy mildew (Plasmopara viticola) and on phytoalexin synthesis in different grapevine varieties. Vitis 26:192-200.

Bavaresco, L., D. Petegolli, E. Cantu, M. Fregoni, G. Chiusa \& M. Trevisan (1997). Elicitation and accumulation of stilbene phytoalexins in grapevine berries infected by Botrytis cineare. Vitis 36(2):77-83.

Bavaresco, L., S. Pezzutto, A. Ragga, F. Ferrari \& M. Trevisan (2001). Effect of nitrogen supply on trans-resveratrol concentration in berries of Vitis vinifera L. Cv. Cabernet Sauvignon. Vitis 40(4):229-230.

Bavaresco, L., S. Vezzulli, P. Battilani, P. Giorni, A. Pietri \& T. Bertuzzi (2003). Effect of ochratoxin A-producing Aspergilli on stilbenic phytoalexin synthesis in grapes. Journal of Agricultural and Food Chemistry 51:6151-6157. https://doi.org/10.1021/jf0301908

Beejmohun V., O. Fliniaux, E. Grand, F. Labmlin, L. Bensaddek, P. Christen, J. Kovensky, M-A. Fliniaux \& F. Mesnard (2007). Microwave-assisted extraction of the main phenolic compounds in flaxseed. Phytochemical Analysis 18(4):275-282. https://doi.org/10.1002/pca.973

Beggs, C.J., Stolzer-Jehle, A. \& E. Wellmann (1985). Isoflavonoid formation as an indicator of UV stress in bean (Phaseolus vulgaris L.) leaves. Plant Physiology 79(3):630-634. https://doi.org/10.1104/pp.79.3.630

Begum A.N., C. Nicolle, I. Mila, C. Lapierre, K. Nagano, K. Fukushima, S-M. Heinonen, H. Adlercreutz, C. Rémésy \& A. Scalbert (2004). Dietary lignins are precursors of mammalian lignans in rats. The Journal of Nutrition 134(1):120-127.

Belisario-Sánchez, Y.Y., A. Taboada-Rodríguez, F. Marín-Iniesta \& A. López-Gómez (2009). Dealcoholized wines by spinning cone column distillation: phenolic compounds and antioxidant activity measured by the 1,1-diphenyl-2-picrylhydrazyl method. Journal of Agricultural and Food Chemistry 57(15):6770-6778. https://doi.org/10.1021/jf9003879, http://www.ncbi.nlm.nih.gov/pubmed/19588980

Bennett, J.O., O. YU, L.G. Heatherly, \& H.B. Krishnan (2004) Accumulation of genistein and daidzein, soybean isoflavones implicated in promoting human health, is significantly elevated by irrigation. Journal of Agricultural and Food Chemistry 52:7574-7579.

https://doi.org/10.1021/jfo49133k

Bennetts, H.W., E.J. Underwood \& F.L. Shier (1946). A specific breeding problem of sheep on subterranean clover pastures in Western Australia. The Australian Veterinary Journal 22(1):2-12. https://doi.org/10.1111/j.1751-0813.1946.tb15473.x

Berman, K.H., G.G. Harrigan, S.G. Riordan, M.A. Nemeth, C. Hanson, M. Smith, R. Sorbet, E. Zhu \& W.P. Ridley (2009). Compositions of seed, forage, and processed fractions from insectprotected soybean MON 87701 are equivalent to those of conventional soybean. Journal of Agricultural and Food Chemistry 57(23):11360-11369.

http://www.ncbi.nlm.nih.gov/pubmed/19891479

Berman, K.H., G.G. Harrigan, S.G. Riordan, M.A. Nemeth, C. Hanson, M. Smith, R. Sorbet, E. Zhu \& W.P. Ridley (2010). Compositions of forage and seed from second-generation glyphosate-tolerant soybean MON 89788 and insect-protected soybean MON 87701 from Brazil are equivalent to those of conventional soybean (Glycine max). Journal of Agricultural and Food Chemistry 58(10):6270-6276. https://doi.org/10.1021/jf902955r,

http://www.ncbi.nlm.nih.gov/pubmed/20420455

Berman, K.H., Harrigan, G.G., Nemeth, M.A., Oliveira, W.S, Berger, G.U. \& F.S. Tagliaferro (2011). Compositional equivalence of insect-protected glyphosate-tolerant soybean MON $87701 \times$ MON 89788 to conventional soybean extends across different world regions and multiple growing seasons. Journal of Agricultural and Food Chemistry 59(21):11643-11651. https://doi.org/10.1021/jf202782z, http://www.ncbi.nlm.nih.gov/pubmed/21985102

Bickoff E.M., A.L. Livingston, A.P. Hendrickson \& A.N. Booth (1962). Relative potencies of several estrogen-like compounds found in forages. Agricultural and Food Chemistry 10(5):410412. https://doi.org/10.1021/jf60123a018

Blaich, R. \& O. Bachmann (1980). Die Resveratrolsynthese bei Vitaceen. Induction and zytologische Beobachtungen Vitas 19:230-340. 
Bolger, R., T. Wiese, K. Ervin, S. Nestich \& W. Checovich (1998). Rapid screening of environmental chemicals for estrogen receptor binding capacity. Environmental Health Perspectives 106(9):551-557. https://doi.org/10.1289/ehp.98106551

Bolling, B.W., C.-Y. O. Chen, D.L. McKay \& J.B. Blumberg (2011). Tree nut phytochemicals: composition, antioxidant capacity, bioactivity, impact factors. A systematic review of almonds, Brazils, cashews, hazelnuts, macadamias, pecans, pine nuts, pistachios and walnuts. Nutrition Research Reviews 24:244-275. https://doi.org/10.1017/So95442241100014X

Booth, N.L., C.R. Overk, P. Yao, S. Totura, Y. Deng, A.S. Hedayat, J.L. Bolton, G.F. Pauli \& N.R. Farnsworth (2006). Seasonal variation of red clover (Trifolium pratense L., Fabaceae) isoflavones and estrogenic activity. Journal of Agricultural and Food Chemistry 54:1277-1282. https://doi.org/10.1021/jfo52927U

Borie, B., P. Jeandet, R. Bessis \& M. Adrian (2004). Comparison of resveratrol and stilbene synthase mRNA production from grapevine leaves treated with biotic and abiotic phytoalexin elicitors. Amerian Journal of Enology and Viticulture 55:60-64.

Borowska, E.J., B. Mazur, R.G. Kopciuch \& B. Buszewski (2009). Polyphenol, anthocyanin and resveratrol mass fractions and antioxidant properties of craneberry cultivars. Food Technology and Biotechnology 47(1):56-61.

Boué, S.M., Carter, C.H., Ehrlich, K.C. \& T.E. Cleveland (2000). Induction of the soybean phytoalexins coumestrol and glyceollin by aspergillus. Journal of Agricultural and Food Chemistry 48(6):2167-2172. https://doi.org/10.1021/jf9912809

Boue, S.M., B.Y. Shih, M.E. Burow, G. Eggleston, S. Lingle, Y.-B. Pan, K. Daigle \& D. Bhatnagar (2013). Postharvest accumulation of resveratrol and piceatannol in sugarcane with enhanced antioxidant activity. Journal of Agricultural and Food Chemistry 61:8412-8419. https://doi.org/10.1021/jfo256384

Brandolini, V., A. Maietti, P. Tedeschi, E. Durini, S. Vertuani \& S. Manfredini (2002). Capillary electrophoresis determination, synthesis, and stability of resveratrol and related $3-O-\beta-D-$ glucopyranoside. Journal of Agricultural and Food Chemistry 50:7407-7411. https://doi.org/10.1021/jfo256384

Brenes, M., A. Garcia, M.C. Dobarganes, J. Velasco \& C. Romero (2002). Influence of thermal treatments simulating cooking processes on the polyphenol content in virgin olive oil. Journal of Agricultural and Food Chemistry 50:5962-5967. https://doi.org/10.1021/jfo20506w

Breuil, A.-C., M. Adrian, N. Pirio, P. Meunier, R. Bessis \& P. Jeandet (1998). Metabolism of stilbene phytoalexins by Botrytis cinearea: 1 . Characterization of a resveratrol dehydrodimer. Tetrahedron Letters 39:537-540. https://doi.org/10.1016/S0040-4039(97)10622-0

Burns, J., P.T. Gardner, J. O’Neil, S. Crawford, I. Morecroft, D.B. McPhail, C. Lister, D. Matthews, M.R. MacLean, M.E.J. Lean, G.G. Duthie \& A. Crozier (2000). Relationship among antioxidant activity, vasodilation capacity, and phenolic content of red wines. Journal of Agricultural and Food Chemistry 48:220-230. https://doi.org/10.1021/jf9909757

Burns, J., P.T. Gardner, D. Matthews, M.R., G.G. Ditjoe, M.E.J. Lean \& A. Crozier (2001). Extraction of phenolics and changes in antioxidant activity of red wines during vinification. Journal of Agricultural and Food Chemistry 49:5797-5808. https://doi.org/10.1021/jfo10682p

Burns, J., T. Yokota, H. Ashihara, M.E.J. Lean \& A. Crozier (2002). Plant foods and herbal sources of resveratrol. Journal of Agricultural and Food Chemistry 50:3337-3340. https://doi.org/10.1021/jfo112973

Cai L., J.A. Koziel, M. Dharmadhikari \& J.H. van Leeuwen (2009). Rapid determination of transresveratrol in red wine by solid-phase microextraction with on-fiber derivatization and multidimensional gas chromatography-mass spectrometry. Journal of chromatography $A$ 1216:281-287. https://doi.org/10.1016/j.chroma.2008.11.050

Caldwell, C.R., Britz, S.J. \& R.M. Mirecki (2005). Effect of temperature, elevated carbon dioxide, and drought during seed development on the isoflavone content of dwarf soybean [Glycine max (L.) Merrill] grown in controlled environments. Journal of Agricultural and Food Chemistry 53:1125-1129. https://doi.org/10.1021/jfo355351 
Callemien, D., V. Jerkovic, R. Rozenberg \& S. Collin (2005). Hop as an interesting source of resveratrol for brewers: Optimization of the extraction and quantitative study by liquid chromatography/atmospheric pressure chemical ionization tandem mass spectrometry. Journal of Agricultural and Food Chemistry 53:424-429. https://doi.org/10.1021/jfo40179n

Cantos, E., C. García-Viguera, S. de Pascual-Teresa \& F.A. Tomás-Barberán (2000). Effect of postharvest ultraviolet irradiation on resveratrol and other phenolics of cv. Napoleon table grapes. Journal of Agricultural and Food Chemistry Journal of Agricultural and Food Chemistry 48(10):4606-4612. https://doi.org/10.1021/jfooo2948

Cantos, E., J.C. Espín \& F.A. Tomás-Barberán (2001). Postharvest induction modeling method using UV irradiation pulses for obtaining resveratrol-enriched table grapes: a new "functional" fruit? Journal of Agricultural and Food Chemistry 49(10):5052-5058.

https://doi.org/10.1021/jfo10366a, http://www.ncbi.nlm.nih.gov/pubmed/11600065

Cantos, E., J.C. Espín \& F.A. Tomás-Barberán (2002). Postharvest stilbene-enrichment of red and white table grape varieties using UV-C irradiation pulses. Journal of Agricultural and Food Chemistry 50(22):6322-6329. https://doi.org/10.1021/jfo20562x, http://www.ncbi.nlm.nih.gov/pubmed/12381111

Cantos, E., J.C. Espín, M.J. Fernández, J. Oliva \& F.A. Tomás-Barberán (2003a). Postharvest UVC-irradiated grapes as a potential source for producing stilbene-enriched red wines. Journal of Agricultural and Food Chemistry 51(5):1208-1214. https://doi.org/10.1021/jfo20939z, http://www.ncbi.nlm.nih.gov/pubmed/12590457

Cantos, E., F.A. Tomás-Barberàn, A. Martinez \& J.C. Espin (2003b). Differential stilbene induction susceptibility of seven red wine grape varieties upon post-harvest UV-C irradiation. European Food Research and Technology 217:253-258. https://doi.org/10.1007/s00217-0030736-x

Carando, S., P.L. Teissedre, P. Waffo-Téguo, J.C. Cabanis, G. Deffieux \& J.M. Mérillon (1999). High-performance liquid chromatrography coupled with fluorescence detection for the determination of trans-astringin in wine. Journal of Chromatography A 849:617-620. https://doi.org/10.1016/S0021-9673(99)00595-6

Careri, M., C. Corradini, L. Elviri, I. Nicoletti \& I. Zagnoni (2003). Direct HPLC analysis of quercetin and trans-resveratrol in red wine, grape, and winemaking byproducts. Journal of Agricultural and Food Chemistry 51(18):5226-5231. https://doi.org/10.1021/jfo34149g, http://www.ncbi.nlm.nih.gov/pubmed/12926863

Careri, M., C. Corradini, L. Elviri, I. Nicoletti \& I. Zagnoni (2004). Liquid chromatographyelectrospray tandem mass spectrometry of cis-resveratrol and trans-resveratrol: development, validation, and application of the method to red wine, grape, and winemaking byproducts. Journal of Agricultural and Food Chemistry 52(23):6868-6874.

https://doi.org/10.1021/jfo49219d, http://www.ncbi.nlm.nih.gov/pubmed/15537288

Carpenter, J.E. (2001). Case studies in benefit and risks of agricultural biotechnology: Roundup Ready soybeans and Bt field corn. National Center for Food and Agricultural Policy, Washington D.C., pp. 1-54.

Carrão-Panizzi, M.C. \& K. Kitamura (1995). Isoflaone content in Brazilian soybean cultivars. Breeding Science 45:295-300.

Carrão-Panizzi, M.C., A. Del Pino Beléia, K. Kitamura \& M.C. Neves Oliveira (1999). Effects of genetics and environment on isoflavone content of soybean from different regions of Brazil. Pesquisa Agropecuária Brasileira 34(10):1788-1795. https://doi.org/10.159o/So100204X1999001000004

Casas, L., C. Mantell, M. Rodríguez, E.J. Martinez de la Ossa, A. Roldán, I.D. Ory, I. Caro \& A. Blandino (2010). Extraction of resveratrol from the pomace of Palomino fino grapes by supercritical carbon dioxide. Journal of Food Engineering 96:304-308.

https://doi.org/10.1016/j.jfoodeng.2009.08.002

Cassidy, A., B. Hanley \& R.M. Lamuela-Raventos (2000). Isoflavones, lignans and stilbenes origins, metabolism and potential importance to human health. Journal of the Science of Food and Agriculture 80:1044-1062. https://doi.org/10.1002/(SICl)1097-

0010(20000515)80:7\%3C1044::AID-JSFA586\%3E3.0.CO;2-N 
Castellari, M., U. Spinabelli, C. Riponi \& A. Amati (1998). Influence of some technological practices on the quantity of resveratrol in wine. Zeitschrift für Lebensmittel-Untersuchung und Forschung A 206:151-155. https://doi.org/10.1007/s002170050232

Celotti, E., R. Ferrarini, R. Zironi \& L.S. Conte (1996). Resveratrol content of some wines obtained from dried Valpolicella grapes: Recioto and Amarone. Journal of Chromatography $A$ 730(1-2):47-52. https://doi.org/10.1016/0021-9673(95)oog62-0, http://www.ncbi.nlm.nih.gov/pubmed/8680595

Chafer, A., M.C. Pascual-Marti, A. Salvador \& A. Berna (2005). Supercritical fluid extraction and HPLC determination of relevant polyphenolic compounds in grape skin. Journal of Separation Science 28:2050-2056. https://doi.org/10.1002/jssc.200500128

Chen, T., J. Li, J. Cao, Q. Xu, K. Komatsu \& T. Namba (1999). A new flavanone isolatd from rhizoma smilais glabrae and the structural requirements of its derivatives from preventing immunological hepatocyte damage. Planta Medica 65:56-59. https://doi.org/10.1055/s-199913963

Chiari, L., Piovesan, N.D., Naoe, L.K., José, I.C., Soriano Viana, J.M., Moreira, M.A. \& E.G. de Barros (2004). Genetic parameters relating isoflavone and protein content in soybean seeds. Euphytica 138:55-60. https://doi.org/10.1023/B:EUPH.0000047060.03101.4a

Chiva-Blanch, G., M. Urpi-Sarda, M. Rotchés-Ribalta, R. Zamora-Ros, R. Llorach, R.M. LamuelaRaventós, R. Estruch \& C. Andrés-Lacueva (2011). Determination of resveratrol and piceid in beer matrices by solid-phase extraction and liquid chromatography-tandem mass spectrometry. Journal of Chromatography A 1218(5):698-705. https://doi.org/10.1016/j.chroma.2010.12.012, http://www.ncbi.nlm.nih.gov/pubmed/21196008

Cho, M.J. \& J.E. Harper (1991a). Effect of inoculation and nitrogen on isoflavonoid concentration in wild-type and nodulaltion-mutant soybean roots. Plant Physiology 95:435-442.

https://doi.org/10.1104/pp.95.2.435

Cho, M.J. \& J.E. Harper (1991b). Effect of localized nitrate application on isoflavonoid concentration and nudulation in split-root systems of wild-type and nudulation-mutant soybean plants. Plant Physiology 95:1106-1112. https://doi.org/10.1104/pp.95.4.1106

Cho, M.J. \& J.E. Harper (1991C). Root isoflavonoid response to grafting between wild-type and nodulation-mutant soybean plants. Plant Physiology 96:1277-1282.

https://doi.org/10.1104/pp.96.4.1277

Choi, S.W., Y.J. Jang, Y.J. Lee, H.H. Leem \& E.O. Kim (2013). Analysis of Functional Constituents in Mulberry (Morus alba L.) Twigs by Different Cultivars, Producing Areas, and Heat Processings. Preventive Nutrition and Food Science 18(4):256-262. https://doi.org/10.3746/pnf.2013.18.4.256, http://www.ncbi.nlm.nih.gov/pubmed/24551827

Chong J., A. Poutaraud \& P. Hugueney (2009). Metabolism and roles of stilbenes in plants. Plant Science 177(3):143-155. https://doi.org/10.1016/j.plantsci.2009.05.012

Chu, Q., M. O'Dwyer \& M.G. Zeece (1998). Direct analysis of resveratrol in wine by micellar electrokinetic capillary electrophoresis. Journal of Agricultural and Food Chemistry 46:509-513. https://doi.org/10.1021/jf970669y

Chukwumah, Y., L.T. Walker, M. Verghese, M. Bokanga, S. Ogutu \& K. Alphonse (2007a). Comparison of extraction methods for the quantification of selected phytochemicals in peanuts (Arachis hypogaea). Journal of Agricultural and Food Chemistry 55:285-290. https://doi.org/10.1021/jfo62148t

Chukwumah, Y., Walker, L., Vogler, B. \& M. Verghese (2007b). Changes in phytochemical composition and profile of raw, boiled, and roasted peanuts. Journal of Agricultural and Food Chemistry 55(22):9266-9273. https://doi.org/10.1021/jfo71877l

Cichewicz, R.H., S.A. Kouzi \& M.T. Hamann (2000). Dimerization of resveratrol by the grapevine pathogen Botrytis cinerea. Journal of Natural Products 63:29-33.

https://doi.org/10.1021/np990266n 
Clare, S.S., G. Skkurray \& R.A. Shalliker (2004). Effect of pomace-contacting method on the concentration of cis- and trans-resveratrol and resveratrol glucoside isomers in wine. American Journal of Enology and Viticulture 55(4):401-406.

Clare, S.S., G.R. Skurray \& R.A: Shalliker (2005). Effect of yeast strain selection on the concentration of cis- and trans-resveratrol and resveratrol glucoside isomers in wine. Australian Journal of Grape and Wine Research 11:9-14. https://doi.org/10.1111/j.17550238.2005.tboo274.x

Clarke, D.B., V. Bailey \& A.S. Lloyd (2008). Determination of phytoestrogens in dietary supplements by LC-MS/MS. Food Additives and Contaminants 25(5):534-547.

Clarke D.B., A.S. Lloyd, J.M. Lawrence, J.E. Brown, L. Storey, M. Raats, R.M. Rainsbury, D.J. Culliford, V.A. Bailey-Horne \& B.M. Parry (2013a). Development of a food compositional database for the estimation of dietary intake of phyto-oestrogens in a group of postmenopausal women previously treated for breast cancer and validation with urinary excretion. British Journal of Nutrition 109(12):2261-2268.

https://doi.org/10.1017/So007114512004394

Clarke, J.D., D.C. Alexander, D.P. Ward, J.A. Ryals, M.W. Mitchell, J.E. Wulff \& L. Guo (2013b) Assessment of genetically modified soybean in relation to natural variation in the soybean seed metabolome. Scientific Reports 3:3082. doi: 10.1038/srepo3082; and doi: 10.1038/srepo3871.

Cooksey, C.J., P.J. Garratt, S.E. Richards \& R.N. Strange (1988). A dienyl stilbene phytoalexin from Arachis hypogaea. Phytochemistry 27(4):1015-1016. https://doi.org/10.1016/00319422(88)80263-2

Cornwell, T., W. Cohick \& I. Raskin (2004). Dietary phytoestrogens and health. Phytochemistry 65:995-1016. https://doi.org/10.1016/j.phytochem.2004.03.005

Cos, P., T. De Bruyne, S. Apers, D. Vanden Berghe, L. Piters \& A.J. Vlietinck (2003). Phytoestrogens: rescent developments. Planta Medica 69(7):589-599.

https://doi.org/10.1055/s-2003-41122

Cosentino M., F. Marino, M. Ferrari, E. Rasini, R. Bombelli, A. Luini, M. Legnaro, M. Gioacchino Delle Canne, M. Luzzani, F. Crema, S. Paracchini \& S. Lecchini (2007). Estrogenic activity of 7hydroxymatairesinol potassium acetate (HMRlignan ${ }^{\mathrm{TM}}$ ) from Norway spruce (Picea abies) knots and of its active metabolite enterolactone in MCF-7 cells. Pharmacological Research 56(2):140-147. https://doi.org/10.1016/j.phrs.2007.05.001

Counet, C., D. Callemien \& S. Collin (2006). Chocolate and cocoa: New sources of transresveratrol and trans-piceid. Food Chemistry 98:649-657.

https://doi.org/10.1016/j.foodchem.2005.06.030

Coward, L., N.C. Barnes, K.D.R. Setchell \& S. Barnes (1993). Genistein, daidzein, and their $\beta$ glycoside conjugates: Antitumor isoflavones in soybean foods from American and Asian diets. Journal of Agricultural and Food Chemistry 41:1961-1967. https://doi.org/10.1021/jfooo35a027

Coward, L., M. Smith, M. Kirk \& S. Barnes (1998). Chemical modification of isoflavones in soyfoods during cooking and processing. American Journal of Clinical Nutrition 68(Suppl.):1486S-1491S.

Creasy, L.L. \& M. Coffee (1988). Phytoalexin production potential of grape berries. Journal of the American Society for Horticultural Science 113:230-234.

Cvejić, J., Malenčič, D., Tepavčević, V., Poša, M. \& J. Miladinović (2009). Determination of phytoestrogen composition in soybean cultivars in Serbia. Natural Product Communications 4(8):1069-1074.

Cvejic, J.M., S.V. Djekic, A.V. Petrovic, M.T. Atanackovic, S.M. Jovic, I.D. Brceski \& L.C. Gojkovic-Bukarica (2010). Determination of trans- and cis-resveratrol in Serbian commercial wines. Journal of Chromatografical Science 48(3):229-234.

https://doi.org/10.1093/chromsci/48.3.229, http://www.ncbi.nlm.nih.gov/pubmed/20223091 
Dani, C., L.S. Oliboni, R. Vanderlinde, D. Bonatto, M. Salvador \& J.A. Henriques (2007). Phenolic content and antioxidant activities of white and purple juices manufactured with organically- or conventionally-produced grapes. Food and Chemical Toxicology 45(12):2574-2580.

https://doi.org/10.1016/j.fct.2007.06.022, http://www.ncbi.nlm.nih.gov/pubmed/17683842

de Andrés-de Prado, R., M. Yuste-Rojas, X. Sort, C. Andrés-Lacueva, M. Torres \& R.M. LamuelaRaventós (2007). Effect of soil type on wines produced from Vitis vinifera L. Cv. Grenache in commercial vineyards. Journal of Agricultural and Food Chemistry 55:779-786.

https://doi.org/10.1021/jfo62446q

De Freitas, A.A., G. de Freitas Hirata, C.D. Tonhi, J.M. Correia da Costa \& E. Clemente (2009). Resveratrol contents found in grape juice extracted from Vitis sp. Varieties and produced through organic and conventional cultivation systems. Journal of Food Technology 7(3):98-101.

Dekić, S., S. Milosavljević, V. Vajs, S. Jović, A. Petrović, N. Nikiċević, V. Manojlovic, V. Nedovic் \& V. Teševic (2008). Trans- and is-resveratrol concentration in wines produced in Serbia. J. Serb. Chem Soc. 73(11):1027-1037. https://doi.org/10.2298/JSC0811027D

Delannay, X., T.T. Bauman, D.H. Beighley, M.J. Buettner, H.D. Cole, M.S. DeFelice, C.W. Dertomg, T.J. Diedrick, J.L. Griffin, E.S. Hagood, F.G. Hancock, S.E. Hart, B.J. LaVallee, M.M. Loux, W.E. Lueschen, K.W. Matson, C.K. Moots, E. Murdock, A.D. Nickell, M.D.K. Owen, E.H. Paschal II, L.M. Prochaska, P.J. Raymond, D.B. Reynolds, W.K. Rhodes, F.W. Roeth, P.L. Sprankle, L.J. Tarochione, C.N. Tinius, R.H. Walker, L.M. Wax, H.D. Weigelt \& S.R. Padgette (1995). Yield evaluation of a glyphosate-tolerant soybean line after treatment with glyphosate. Crop Science 35:1461-1467. https://doi.org/10.2135/cropsci1995.0011183X003500050033X

Della-Cioppa, G., S.C. Baver, B.K. Klein, D.M. Shah, R.T. Fraley \& G.M. Kishore (1986) Translocation of the precursor of 5-enolpyruvylshikimate-3-phosphate synthase into chloroplasts of higher plants in vitro. Proceedings of the National Academy of Science 83:68736877. https://doi.org/10.1073/pnas.83.18.6873

DellaGreca M., S. Zuppolini \& A. Zarrelli (2013). Isolation of lignans as seed germination and plant growth inhibitors from Mediterranean plants and chemical synthesis of some analogues. Phytochemistry Reviews 12(4):717-731. https://doi.org/10.1007/s11101-013-9311-7

de Lima, P.F., C.A. Colombo, A.F. Chiorato, L.F. Yamaguchi, M.J. Kato \& S.A.M. Carbonell (2014). Occurrence of isoflavonoids in Brazilian common bean germplasm (Phaseolus vulgaris L.). Journal of Agricultural and Food Chemistry 62(4):9699-9704. https://doi.org/10.1021/jf5033312

De Mejia, E.G., S.H. Guzmán-Maldonado, J.A. Acosta-Gallegos, R. Reynoso-Camacho, E. Ramirez-Rodriguez, J.L. Pons-Gernández, M.M. González-Chavira, J.Z. Castellanos \& J.D. Kelly (2003). Effect of cultivar and growing location on the trypsin inhibitors, tannins, and lections of Common beans (Phaseolus vulgaris L.) grown in the semiarid highlands of Mexico. Journal of Agricultural and Food Chemistry 51:5962-5966. https://doi.org/10.1021/jfo30046m

De Nisco, M., M. Manfra, A. Bolognese, A. Sofo, A. Scopa, G.C. Tenore, F. Pagano, C. Milite \& M.T. Russo (2013). Nutraceutical properties and polyphenolic profile of berry skin and wine of Vitis vinifera L. (cv. Aglianico). Food Chemistry 140(4):623-629.

https://doi.org/10.1016/j.foodchem.2012.10.123,

http://www.ncbi.nlm.nih.gov/pubmed/23692745

Dercks, W. \& L.L. Creasy (1989). The significance of stilbene phytoalexins in the Plasmopara viticola-grapevine interaction. Physiological and Molecular Plant Pahtology 34:189-202. https://doi.org/10.1016/0885-5765(89)90043-X

De Rossi, P., A. Ricelli, M. Reverberi, C. Bello, A.A. Fabbri, C. Fanelli, A. De Rossi, D. Corradini \& I. Nicoletti (2012). Grape variety related trans-resveratrol induction affects Aspergillus carbonarius growth and ochratoxin A biosynthesis. International Journal of Food Microbiology 156(2):127-132. https://doi.org/10.1016/j.ijfoodmicro.2012.03.013, http://www.ncbi.nlm.nih.gov/pubmed/22483545

Deshpande, V.H., R. Srinivasan \& A.V. Rama Rao (1978). Wood phenolics of Morus species: Part IV - Phenolics of the heartwood of five Morus species. Indian Journal of Chemistry 13:453-457. 
Devi, M.K.A., M. Gondi, G. Sakthivelu, P. Giridhar, T. Rajasekaran \& G.A. Ravishankar (2009). Functional attributes of soybean seeds and products, with reference to isoflavone content and antioxidant activity. Food Chemistry 114:771-776.

https://doi.org/10.1016/j.foodchem.2008.10.011

Dewick, P.M. (1988). Isoflavonoids. In: The Flavonoids. Advances in Research since 1980; J.B. Harborne (Ed.), Chapman and Hall, London/New York, pp. 125-209.

https://doi.org/10.1007/978-1-4899-2913-6_5

Diaz-Batalla, L., Widholm, J.M., Fahey, G.C. Jr., Castaño-Tostado, E. \& O. Paredes-López (2006). Chemical components with health implications in wild and cultivated Mexican common bean seeds (Phaseolus vulgaris L.). Journal of Agricultural and Food Chemistry 54(6):2045-2052. https://doi.org/10.1021/jf051706l

Dinelli G., I. Marotti, S. Bosi, S. Benedettelli, L. Ghiselli, S. Cortacero-ramírez, A. CarrascoPancorbo, A. Segura-Carretero \& A. Fernández-Gutiérrez (2007). Lignan profile in seeds of modern and old Italian soft wheat (Triticum aestivum L.) cultivars as revealed by CE-MS analyses. Electrophoresis 27(22):4212-4219. https://doi.org/10.1021/jfo51706l

Dixon, R.A. (2004). Phytoestrogens. Annual Review of Plant Biology 55(1):225-261. https://doi.org/10.1146/annurev.arplant.55.031903.141729

Dixon, R.A. \& N.L. Paiva (1995). Stress-induced phenylpropanoid metabolism. The Plant Cell 7:1085-1097. https://doi.org/10.1105/tpc.7.7.1085

Dolatabadian, A., Modarres Sanavy, S.A.M., Ghanati, F. \& P.M. Gresshoff (2013). Agrobacterium rhizogenes transformed soybean roots differ in their nodulation and nitrogen fixation response to genistein and salt stress. World Journal of Microbiology and Biotechnology 29:1327-1339. https://doi.org/10.1007/s11274-013-1296-2

Dorner, J.W., R.J. Cole, T.H. Sanders \& P.D: Blankenship (1989). Interrelationship of kernel water activity, soil temperature, maturity, and phytoalexin contamination of drought-stressed peanuts. Mycopathologia 105:117-128. https://doi.org/10.1007/BFo0444034

Douillet-Breuil, A.C., P. Jeandet, M. Adrian \& R. Bessis (1999). Changes in the phytoalexin content of various Vitis spp. in response to ultraviolet $C$ elicitation. Journal of Agricultural and Food Chemistry 47(10):4456-4461. https://doi.org/10.1021/jf9900478,

http://www.ncbi.nlm.nih.gov/pubmed/10552833

Dourtoglou, V.G., D.P. Makris, F. Bois-Dounas \& C. Zonas (1999). Trans-Resveratrol concentration in wines produced in Greece. Journal of Food Composition and Analysis 12:227233. https://doi.org/10.1006/jfca.1999.0821

Durazzo A., V. Turfani, E. Azzini, G. Maiani \& M. Carcea (2013). Phenols, lignans and antioxidant properties of legume and sweet chestnut flours. Food Chemistry 14O(4):666-671. https://doi.org/10.1016/j.foodchem.2012.09.062

Durazzo A., V. Turfani, V. Narducci, E. Azzini, G. Mainani \& M. Carcea (2014). Nutritional characterisation and bioactive components of commercial carob flours. Food Chemistry 153:109-113. https://doi.org/10.1016/j.foodchem.2013.12.045

Ector, B.J., J.B. Magee, C.P. Hegwood \& M.J. Coign (1996). resveratrol concentration in muscadine berries, juice, pomace, purees, seeds, and wine. American Journal of Enology and Viticulture 47(1):57-62.

Eder, R., S. Wendelin \& U. Vrhovsek (2001). Resveratrolgehalte von Trauben und Rotweinen in Abhängigkeit von Lesejahrgang und Lesetermin. Mitteilungen Klosterneuburg 51:64-78.

EFSA (2004). Opinion of the scientific panel on contaminants in the food chain on a request from the commission related to zearalenone as undesirable substance in animal feed. EFSA Journal 2004:89: $35 \mathrm{pp}$.

EFSA (2010). EFSA Panel on Genetically Modified Organisms. Scientific opinion on statistical considerations for the safety evaluation of GMOs. EFSA Journal 2010; 8(1):1250, 59 pp.

EFSA (2011). EFSA Panel on Genetically Modified Organisms. Guidance for risk assessment of food and feed from genetically modified plants. EFSA Journal 2011; 9(5):2150, 37 pp.

EFSA (Applications). http://www.efsa.europa.eu/en/applicationshelpdesk 
Eisen, B., Ungar, Y. \& E. Shimoni (2003). Stability of isoflavones in soy milk stored at elevated and ambient temperatures. Journal of Agricultural and Food Chemistry 51:2212-2215. https://doi.org/10.1021/jfo25783h

Eldridge, A.C. (1982). Determination of isoflavones in soybean flours, protein concentrates, and isolates. Journal of Agricultural and Food Chemistry 30, 353-355.

https://doi.org/10.1021/jfoo110a035

Eldridge, A.C. \& W.F. Kwolek (1983). Soybean isoflavones: Effect of environment and variety on composition. Journal of Agricultural and Food Chemistry 31:394-396.

https://doi.org/10.1021/jfoo116ao52

Eliasson C., A. Kamal-Eldin, R. Andersson \& P. Åman (2003). High-performance liquid chromatographic analysis of secoisolariciresinol diglucoside and hydroxycinnamic acid glucosides in flaxseed by alkaline extraction. Journal of Chromatography 1012(2):151-159. https://doi.org/10.1016/S0021-9673(03)01136-1

Erdtman, H. (1939) Die Phenolischen Inhaltsstoffe des Kieferkernholtzes, ihre Physiologische Bedeutung und hemmende Einwirkungauf die normale Augschlissbarkeit des Kiefernkernholzes nach dem Sulfitverfahren. Julius Liebigs Annalen der Chemie 539:116-127. https://doi.org/10.1002/jlac.19395390111

Farmakalidis E. \& P.A. Murphy (1984). Oestrogenic response of the CD-1 mouse to the soyabean isoflavones genistein, genistin and daidzin. Food and Chemical Toxicology 22(3):237-239. https://doi.org/10.1016/0278-6915(84)90134-0

Farmakalidis E., J.N. Hathcock \& P.A. Murphy (1985). Oestrogenic potency of genistin and daidzin in mice. Food and Chemical Toxicology 23(8):741-745. https://doi.org/10.1016/02786915(85)90268-6

Fast, B.J., Schafer, A.C., Johson, T.Y., Potts, B.L. \& R.A. Herman (2015). Insect-protected event DAS-81419-2 soybean (Glycine max (L.) grown in the United States and Brazil is compositionally equivalent to nontransgenic soybean. Journal of Agricultural and Food Chemistry 63:2063-2073. https://doi.org/10.1021/jf505015y

Fehily, A.M. (2003). Dietary importance. Encyclopedia of Food Sciences and Nutrition, p. 53925398. https://doi.org/10.1016/Bo-12-227055-X/01112-3

Feijóo, O., A. Moreno \& E. Falqué (2008). Content of trans- and cis-resveratrol in Galician white and red wines. Journal of Food Composition and Analysis 21:608-613.

https://doi.org/10.1016/j.jfca.2008.06.002

Fernández-Marín, M.I., R.F.Guerrero, M.C. García-Parrilla, B. Puertas, p. Ramírez \& E. CantosVillar (2013). Terroir and variety: Two key factors for obtaining stilbene-enriched grapes.

Journal of Food Composition and Analysis 31:191-198.

https://doi.org/10.1016/j.jfca.2013.05.009

Fernández-Marín, M.I., B. Puertas, R.F.Guerrero, M.C. García-Parrilla \& E. Cantos-Villar (2014). Preharvest methyl jasmonate and postharvest UVC treatments: increasing Stilbenes in wine. Journal of Food Science 79(3):C310-C317. https://doi.org/10.1111/1750-3841.12368, http://www.ncbi.nlm.nih.gov/pubmed/24498957

Figueiredo-González, M., J. Regueiro, B. Cancho-Grande \& J. Simal-Gándara (2014). Garnacha Tintorera-based sweet wines: detailed phenolic composition by HPLC/DAD-ESI/MS analysis. Food Chemistry 143:282-292. https://doi.org/10.1016/j.foodchem.2013.07.120, http://www.ncbi.nlm.nih.gov/pubmed/24054241

File, S.E., N. Jarrett, E. Fluck, R. Duffy, K. Casey \& H. Wiseman (2001). Eating soya improves human memory. Psychopharmacology 157(4):430-436. https://doi.org/10.1007/s002130100845

Filip, V., M. Plpclpvá, J. Šmidrkal, Z. Špičková, K. Melzoch \& Š. Schmidt (2003). Resveratrol and its antioxidant and antimicrobial effectiveness. Food Chemistry 83:585-593. https://doi.org/10.1016/S0308-8146(03)00157-2

Flamini, R. (2003). Mass spectrometry in grape and wine chemistry. Part 1: Polyphenols. Mass Spectrometry Reviews 22:218-250. https://doi.org/10.1002/rcm.1569 
Flamini, R. \& A. Dalla Vedova (2004). Fast determination of the total free resveratrol content in wine by direct-exposure-probe, positive-ion chemical ionization and collision-induceddissociation mass spectrometry. Rapid Communications in Mass Spectrometry 18(17):19251931. https://doi.org/10.1002/rcm.1569, http://www.ncbi.nlm.nih.gov/pubmed/15329858

Franco, M.A., G.C. Coloru, A. Del Caro, G. Emonti, G.A. Farris, G. Manca, T.G. Massa \& G. Pinna (2002). Variability of resveratrol $\left[3,5,4^{\prime}\right.$-trihydroxystilbene] content in relation to the fermentation processes by Saccharomyces cerevisiae strains. European Food Research and Technology 214:221-225. https://doi.org/10.1007/s00217-001-0436-3

Franke, A.A., Custer, H.-S., Cerna, C.M. \& K.K. Narala (1994). Quantitation of phytoestrogens in legumes by HPLC. Journal of Agricultural and Food Chemistry 42(9):1905-1913.

https://doi.org/10.1021/jfooo45a015

Franzmayr, B.K., Rasmussen, S., Fraser, K.M. \& P.E. Jameson (2012). Expression and functional characterisation of a white clover isoflavone synthase in tobacco. Annals of Botany 110:12911301. https://doi.org/10.1093/aob/mcs168

Fregoni, M., L. Bavaresco, D. Petegolli, M. Trevisan \& C. Ghebbioni (1994). Indagine sul contenuto di resveratrolo in aluni vini della Valle d'Aosta e dei colli piacentini. Vignivini $1994(\&): 33-36$.

Frémont, L. (2000). Biological effects of resveratrol. Life Sciences 66:663-673. https://doi.org/10.1016/So024-3205(99)00410-5

FSA (2003). Committee on toxicity of chemicals in food, consumer products and the environment, phytoestrogens and health. Food Standards Agency, UK.

Fukutake, M., M. Takahashi, K. Ishida, H. Kawamura, T. Sugimura \& K. Wakabayashi (1996). Quantification of genistein and genistin in soybeaans and soybean products. Food and Chemical Toxicology 34:457-461. https://doi.org/10.1016/0278-6915(96)87355-8

Gambelli, L. \& G.P. Santaroni (2004). Polyphenols content in some Italian red wines of different geographical origins. Journal of Food Composition and Analysis 17:613-618.

https://doi.org/10.1016/j.jfca.2003.09.010

Gambuti, A., D. Strollo, M. Ugliano, L. Lecce \& L. Moio (2004). trans-Resveratrol, quercetin, (+)catechin, and (-)-epicatechin content in south Italian monovarietal wines: relationship with maceration time and marc pressing during winemaking. Journal of Agricultural and Food Chemistry 52(18):5747-5751. https://doi.org/10.1021/jfo354895,

http://www.ncbi.nlm.nih.gov/pubmed/15373419

Gambuti, A., D. Strollo, A. Erbaggio, L. Lecce \& L. Moio (2007). Effect of winemaking practices on color indexes and selected bioactive phenolics of Aglianico wine. Journal of Food Science 72(9):S623-S628. https://doi.org/10.1111/j.1750-3841.2007.00536.x, http://www.ncbi.nlm.nih.gov/pubmed/18034746

Gao, L., Q. Chu \& J. Ye (2002). Determination of trans-resveratrol in wines, herbs and health food by capillary electrophoresis with electrochemical detection. Food Chemistry 78:255-26o. https://doi.org/10.1016/S0308-8146(02)00115-2

Gatto P., U. Vrhovsek, J. Muth, C. Segala, C. Ramualdi, P. Fontana, D. Pruefer, M. Stefanini, C. Moser, F. Mattivi \& R. Velasco (2008) Ripening and genotype control stilbene accumulation in healthy grapes. Journal of Agricultural and Food Chemistry 56:11773-11785.

https://doi.org/10.1021/jf8017707

Gehm, B.D., J.M. MccAndrews, P.-Y. Chen \& J.L. Jameson (1997) Resveratrol, a polyphenolic compound found in grapes and wine, is an agonist for the estrogen receptor. Proceedings of the National Academy of Sciences 94:14138-14143. https://doi.org/10.1073/pnas.94.25.14138

Genovese, M.I., N.M.A. Hassimotto \& F.M. Lajolo (2005). Isoflavone profile and antioxidant activity in Brazilian soybean varieties. Food Science and Technology International 11(3):205211. https://doi.org/10.1177/1082013205054499

Gerogiannaki-Christopoulou, M., P. Athanasopoulos, N. Kyriakidis, I.A. Gerogiannaki \& M. Spanos (2006). trans-Resveratrol in wines from the major Greek red and white grape varieties. Food Control 17:700-706. https://doi.org/10.1016/j.foodcont.2005.04.006 
Gerstenmeyer E., S.Reimer, E. Berghofer, H. Schwartz \& G. Sontag (2013). Effect of thermal heating on some lignans in flax seeds, sesame seeds and rye. Food Chemistry 138(2-3):18471855. https://doi.org/10.1016/j.foodchem.2012.11.117

Ghisalberti, E.L., P.R. Jefferies, R. Lanteri \& J. Matisons (1978). Constituents of propolis. Experientia 34:157-158. https://doi.org/10.1007/BFo1944648

Gobbi, L., I. Nicoletti, A.M. Tarola \& C. Corradini (2004). Evalustion of the level of cis- and transresveratrol and their glucosides in red wines produced in Lazio region (area). Journal of Commodity Science 43(IV):173-190.

Goda, Y., H. Akiyama, E. Suyama, S. Takahashi, J. Kinjo, T. Nohara \& M. Toyoda (2002). Comparison of soyasaponin and isoflavone contents between genetically modified (GM) and non-GM soybeans. Shokuhin Eiseigaku Zasshi 43(6):339-347.

https://doi.org/10.3358/shokueishi.43.339

Goldberg, D.M., A. Karumanchiri, E. Ng, J. Yan, E.P. Diamandis \& G.J. Soleas (1995a). Direct gas chromatographic-mass spectrometric method to assay cis-resveratrol in wines: Preliminary survey of its concentration in commercial wines. Journal of Agricultural and Food Chemistry 43:1245-1250. https://doi.org/10.1021/jfooo53ao23

Goldberg, D.M., E. Ng, A. Karumanchiri, J. Yan, E.P. Diamandis \& G.J. Soleas (1995b). Assay of resveratrol glucosides and isomers in wine by direct-injection high performance liquid chromatography. Journal of Chromatography A 708:89-98. https://doi.org/10.1016/00219673(95)00368-W

Goldberg, D.M., J. Yan, E. Ng, E.P. Diamandis, A. Karumanchiri, G. Soleas \& A.L. Waterhouse (1995c). A gobal survey of trans-resveratrol concentrations in commercial wines. American Journal of Enology and Viticulture 46(2):159-165.

Goldberg, D.M., E. Tsang, A. Karumanchiri, E.P. Diamandis, G.J. Soleas \& J. Yan (1996a). Method to assay the concentrations of phenolic constituents of biological interest in wines. Analytical Chemistry 68:1688-1694. https://doi.org/10.1021/ac951083i

Goldberg, D.M., E. Ng, A. Karumanchiri, E.P. Diamandis \& G.J. Soleas (1996b). Resveratrol glucosides are important components of commercial wines. American Journal of Enology and Viticulture 47(4):415-420.

Goldberg, D.M., E. Ng, J. Yan, A. Karumanchiri, G.J. Soleas \& E.P. Diamandis (1996c). Regional differences in resveratrol isomer concentrations of wines from various cultivars. Journal of Wine Research 7(1):13-24. https://doi.org/10.1080/09571269608718057

González-Barrio, R., D. Beltrán, E. Cantos, M.I. Gil, J.C. Espín \& F.A. Tomás-Barberán, (2006). Comparison of ozone and UV-C treatments on the postharvest stilbenoid monomer, dimer, and trimer induction in var. 'Superior' white table grapes. Journal of Agricultural and Food Chemistry 54:4222-4228. https://doi.org/10.1021/jfo6o16of

Gorbatsova, J., T. Lõugas, R. Vokk \& M. Kaljurand (2007). Comparison of the contents of various antioxidants of sea buckthorn berries using CE. Electrophoresis 28(22):4136-4142. https://doi.org/10.1002/elps.200700362, http://www.ncbi.nlm.nih.gov/pubmed/17955453

Graham, T.L., J.E. Kim \& M.Y. Graham (1990). Role of constitutive isoflavone conjugates in the accumulation of glyceollin in soybean infected with Phytophthora megasperma. Molecular Plant-Microbe Interactions 3:157-166. https://doi.org/10.1094/MPMI-3-157

Graham, T.L. \& M.Y. Graham (1996). Signaling in soybean phenylpropanoid response: dissection of primary, secondary, and conditioning effects of light, wounding, and elicitor treatments. Plant Physiology 110(4):1123-1133. https://doi.org/10.1104/pp.110.4.1123

Grimmig, B., R. Schubert, R. Fischer, R. Hain, P.H. Schreier, C. Betz; C. Langebartels, D. Ernst \& E'H. Sandermann Jr. (1997) Ozone- and ethylene-induced regulationof a grapevine resveratrol synthase promoter in transgenic tobacco. Acta Physiologiae Plantarum 19(4):467-474. https://doi.org/10.1007/s11738-997-0043-4

Grippi, F., L. Crosta, G. Aiello, M. Tolomeo, F. Oliveri, N. Gebbia \& A. Curione (2008). Determination of stilbenes in Sicilian pistachio by high-performance liquid chromatographic diode array (HPLC-DAD/FLD) and evaluation of eventually mycotoxin contamination. Food Chemistry 107:483-488. https://doi.org/10.1016/j.foodchem.2007.07.079 
Grün, I.U., Adhikari, K., Li, C., Li, Y., Lin, B., Zhang, J. \& L.N. Fernando (2001). Changes in the profile of genistein, daidzein, and their conjugates during thermal processing of tofu. Journal of Agricultural and Food Chemistry 48:2839-2843. https://doi.org/10.1021/jfo10028+

Gu, X., L. Creasy, A. Kester \& M. Zeece (1999). Capillary electrophoretic determination of resveratrol in wines. Journal of Agricultural and Food Chemistry 47:3223-3227.

https://doi.org/10.1021/jfg81211e

Gu, X., Q. Chu, M. O'Dwyer \& M. Zeece (2000). Analysis of resveratrol in wine by capillary electrophoresis. Journal of Chromatography A 881:471-481. https://doi.org/10.1016/Soo219673(00)00211-9

Guerrero, R.F., B. Puertas, M.O. Fernández, M. Palma \& E. Cantos-Villar (2010). Induction of stilbenes in grapes by UV-C: Comparison of different subspecies of Vitis. Innovative Food Science and Emerging Technologies 11:231-238. https://doi.org/10.1016/j.ifset.2009.10.005 Gutierrez-Gonzalez, J.J., Wu, X., Zhang, J., Lee, J.-D., Ellersieck, M., Shannon, J.G., Yu, O., Nguyen, H.T. \& D.A. Sleper (2009). Genetic control of soybean seed isoflavone content: importance of statistical model and epistasis in complex traits. Theoretical and Applied Genetics 119:1069-1083. https://doi.org/10.1007/s00122-009-1109-z

Gutierrez-Gonzalez, J.J., Guttikonda, S.K., Tran, L.-S.P., Aldrich, D.L., Wu, X., Zhong, R, Yu, O., Nguyen, H.T. \& D.A. Sleper (2010). Differential expression of isoflavone biosynthetic genes in soybean during water deficits. Plant Cell Physiology 51(6):936-948.

https://doi.org/10.1093/pcp/pcqo65

Gürbüz, O., D. Göçmen, F. Dağdelen, M. Gürsoy, S. Aydin, I. Şahin, L. Büyükuysal \& M. Usta (2007). Determination of flavan-3-ols and trans-resveratrol in grapes and wine using HPLC with fluorescence detection. Food Chemistry 100:518-525.

https://doi.org/10.1016/j.foodchem.2005.10.008

Hall, D. \& V. De Luca (2007). Mesocarp localization of a bi-functional resveratrol/hydroxycinnamic acid glucosyltransferase of Concord grape (Vitis labrusca). Plant Journal 49:579-591. https://doi.org/10.1111/j.1365-313X.2006.02987.X

Hao M. \& T. Beta (2012). Qualitative and quantitative analysis of the major phenolic compounds as antioxidants in barley and flaxseed hulls using HPLC/MS/MS. Journal of the Science of Food and Agriculture 92(10):2062-2068. https://doi.org/10.1002/jsfa.5582

Harrigan, G.G. \& J.M. Harrison (2012). Assessing compositional variability through graphical analysis and Bayesian statistical approaches: Case studies on transgenic crops. Biotechnology and Genetic Engineering Reviews 28:15-32. https://doi.org/10.5661/bger-28-15

Harrigan, G.G., W.P. Ridley, S.G. Riordan, M.A. Nemeth, R. Sorbet, W.A. Trujillo, M.L. Breeze \& R.W. Schneider (2007). Chemical composition of glyphosate-tolerant soybean 40-3-2 grown in Europe remains equivalent with that of conventional soybean (Glycine max L.). Journal of Agricultural and Food Chemistry 55(15):6160-6168. https://doi.org/10.1021/jfo704920

Harrigan, G.G., Lundry, D., Drury, S., Berman, K., Riordan, S.G., Nemeth, M.A., Glenn, K.C., Ridley, W.P. \& K.C. Glenn (2010a). Natural variation in crop composition and the impact of transgenesis. Nature Biotechnology 28(5): 402-404 and supplementary information.

Harrigan, G.G., Glenn, K.C. \& W.P. Ridley (2010b). Assessing the natural variability in crop composition. Regulatory Toxicology and Pharmacology 58:513-520.

https://doi.org/10.1016/j.yrtph.2010.08.023

Harrison, J.M., Breeze, M.L., Berman, K.H. \& G.G. Harrigan (2013). Bayesian statistical approaches to compositional analyses of transgenic crops. 2. Application and validation of informative prior distributions. Regulatory Toxicology and Pharmacology 65(2):251-258. https://doi.org/10.1016/j.yrtph.2012.12.002

Hart, J.H. (1981). Role of phytostilbenes in decay and disease resistance. Annual Review of Phytopathology 19:437-458. https://doi.org/10.1146/annurev.py.19.090181.002253

Hata, K., K. Baba \& M. Kozawa (1979). Chemical studies on the heartwood of Cassia garrettiana Craib. II. Nonanthraquinonic consituents. Chemical and Pharmacological Bulletin 27(4):984989. https://doi.org/10.1248/cpb.27.984 
Hathway, D.E. \& J.W.T. Seakins (1959). Hydroxystilbenes of Eucalyptus wandoo. Biochemical Journal 72:369-374. https://doi.org/10.1042/bj072036gb

Heinonen S., T. Nurmi, K. Liukkonen, K. Poutanen, K. Wähälä, T. Deyama, S. Nishibe \& H. Adlercreutz (2001). In vitro metabolism of plant lignans: new precursors of mammalian lignans enterolactone and enterodiol. Journal of Agricultural and Food Chemistry 49(7):3178-3186. https://doi.org/10.1021/jfo10038a

Herrmann, K.M. (1983). The common aromatic biosynthetic pathway. In: Amino Acids. Biosynthesis and Genetic Regulation, K.M. Hermann \& R.L. Somerville (Eds.), Addison-Wesley Publishing Company, Reading (MA), pp. 301-322.

Herman, R.A., Phillips, A.M., Lepping, M.D. \& J. Sabbatini (2011). Compositional safety of DAS68416-4 (AAD-12) herbicide-tolerant soybean. Journal of Nutrition \& Food Science 1:103. https://doi.org/10.4172/2155-9600.1000103

Ho, H.M., Chen, R.Y., Leung, L.K., Chan, F.L., Huang, Y. \& Z.-Y. Chen (2002). Difference in flavonoid and isoflavone profile between soybean and soy leaf. Biomedicine \& Pharmacotherapy 56:289-295. https://doi.org/10.1016/S0753-3322(02)00191-9

Hoeck, J.A., W.R. Fehr, P.A. Murphy \& G.A. Welke (2000). Influence of genotype and environment on isoflavone contents of soybean. Crop Science 4O(1):48-51.

https://doi.org/10.2135/cropsci2000.40148x

Hoikkala, A., E. Mustonen, I. Saastamoinen, T. Jokela, J. Taponen, H. Saloniemi \& K. Wähälä (2007). High levels of equol in organic skimmed Finnish cow milk. Molecular Nutrition and Food Research 51:782-786. https://doi.org/10.1002/mnfr.200600222

Holt, S. (1997). Soya: The health food of the next millennium. Korean Soybean Dig 14:77-90. Homsey, C. (1999). Functional Foods and Phytochemicals. Food Product Design Magazine.

Hong, Y.-H., Wang, S.-C., Hsu, C., Lin, B.-F., Kuo, Y.-H. \& C.-J. Huang (2011). Phytoestrogenic compounds in alfalfa sprout (Medicago sativa) beyond coumestrol. Journal of Agricultural and Food Chemistry 59:131-137. https://doi.org/10.1021/jf102997p

Horn-Ross, P.L., S. Barnes, M. Lee, L. Coward, J.E. Mandel, J. Koo, E.M. John \& M. Smith (2000). Assessing phytoestrogen exposure in epidemiologic studies: Development of a database (United States). Cancer Causes and Control 11:289-298. https://doi.org/10.1023/A:1008995606699

Huang, R.-Y. \& C-C. Chou (2008). Heating affects the content and distribution profile of isoflavones in steamed black soybeans and black soybean koji. Journal of Agricultural and Food Chemistry 56(18):8484-8489. https://doi.org/10.1021/jf801488e

Huang, X. \& G. Mazza (2011). Simultaneous analysis of serotonin, melatonin, piceid and resveratrol in fruits using liquid chromatography tandem mass spectrometry. Journal of Chromatography A 1218(25):3890-3899. https://doi.org/10.1016/j.chroma.2011.04.049, http://www.ncbi.nlm.nih.gov/pubmed/21570690

Huang, A., Hsieh, O.A.L. \& S.S. Chang (1981). Characterization of the nonvolatile minor constituents responsible for the objectionable taste of defatted soybean flour. Journal of Food Science 47:19-23. https://doi.org/10.1111/j.1365-2621.1982.tb11017.x

Huang X., Cai, W. \& B. Xu (2014). Kinetic changes of nutrients and antioxidant capacities of germinated soybean (Glycine max L.) and mung bean (Vigna radiate L.) with germination time. Food Chemistry 143:268-276. https://doi.org/10.1016/j.foodchem.2013.07.080

Hubert, J., M. Berger \& J. Dayne (2005). Use of a simplified HPLC-UV analysis for soyasaponin B determination: Study of saponin and isoflavone variability in soybean cultivars and soy-based health food products. Journal of Agricultural and Food Chemistry 53:3923-3930.

https://doi.org/10.1021/jfo47828f

Hyvärinen H.K., J-M. Pihlava, J.A. Hiidenhovi, V. Hietaniemi, H.J.T. Korhonen \& E-L. Ryhänen (2006). Effect of processing and storage on the stability of flaxseed lignan added to bakery products. Journal of Agricultural Food and Chemistry 54(1):48-53.

https://doi.org/10.1021/jf0507590

Ingham, J.I. (1976). 3,5,4'-Trihydroxystilbene as a phytoalexin from groundnuts (Arachis hypogaea). Phytochemistry 15:1791-1793. https://doi.org/10.1016/So031-9422(00)97494-6 
Jaillon, O., J.M. Aury, B. Noel, A. Policriti, C. Clepet, A. Casagrande, N. Choisne, S. Aubourg, N. Vitulo, C. Jubin, A. Vezzi, F. Legeai, P. Hugueney, C. Dasilva, D. Horner, E. Mica, D. Jublot, J. Poulain, C. Bruyère, A. Billault, B. Segurens, M. Gouyvenoux, E. Ugarte, F. Cattonaro, V. Anthouard, V. Vico, C. Del Fabbro, M. Alaux, G. Di Gaspero, V. Dumas, N. Felice, S. Paillard, I. Juman, M. Moroldo, S. Scalabrin, A. Canaguier, I. Le Clainche, G. Malacrida, E. Durand, G. Pesole, V. Laucou, P. Chatelet, D. Merdinoglu, M. Delledonne, M. Pezzotti, A. Lecharny, C. Scarpelli, F. Artiguenave, M.E. Pè, G. Valle, M. Morgante, M. Caboche, A.F. Adam-Blondon, J. Weissenbach, F. Quétier, P. Wincker, \& French-Italian Public Consortium for Grapevine Genome Characterization (2007). The grapevine genome sequence suggests ancestral hexaploidization in major angiosperm phyla. Nature 449:463-467. https://doi.org/10.1038/natureo6148

Jayatilake, G.S., H. Jayasuriya, E.-S. Lee, N.M. Koonchanok, R.L. Geahlen, C.L. Ashendel, J.L. McLaughlin \& C.-J. Chang (1993). Kinase inhibitors from Polygonum cuspidatum. Journal of Natural Products 56(10):1805-1810. https://doi.org/10.1021/np50100a021

Jeandet, P., R. Bessis \& B. Gautheron (1991). The production of resveratrol $\left(3,5,4^{\prime}\right.$ trihydroxystilbene) by grape berries in different developmental stages. American Journal of Enology and Viticulture 42(1):41-46.

Jeandet, P., R. Bessis, B.F. Maume \& M. Sbaghi (1993). Analysis of resveratrol in Burgundy wines. Journal of Wine Research 4(2):79-85. https://doi.org/10.1080/09571269308717954

Jeandet, P., R. Bessis, B.F. Maume, P. Meunier, D. Peyron \& P. Trollat (1995). Effect of enological practices on the resveratrol isomer content of wine. Journal of Agricultural and Food Chemistry 43:316-319. https://doi.org/10.1021/jfooo50a010

Jeandet, P., R. Bessis, M. Sbaghi, P. Meunier \& P. Trollat (1995b). Resveratrol content of wines of different ages: Relationship with fungal disease pressure in the vineyard. American Journal of Enology and Viticulture 46(1):1-4.

Jeandet, P. R. Bessis, M. Sbaghi \& P. Meunier (1995C). Production of the phytoalexin resveratrol by grapes as a response to Botrytis attack under natural conditions. Journal of Phytopathology 143:135-139. https://doi.org/10.1111/j.1439-0434.1995.tboo246.x

Jeandet, P., A.C. Breuil, M. Adrian, L.A: Weston, S. Debord, P. Meunier, G. Maume \& R. Bessis (1997). HPLC analysis of grapevine phytoalexins coupling photodiode array detection and fluorometry. Analytical Chemistry 69:5172-5177. https://doi.org/10.1021/ac970582b

Jeandet, P., B. Delaunois, A. Conreux, D. Donnez, V. Nuzzo, S. Cordelier, C. Clément \& E. Courot (2010). Biosynthesis, metabolism, molecular engineering, and biological functions of stilbene phytoalexins in plants. BioFactors 36(5):331-341. https://doi.org/10.1002/biof.108

Jefferson W.N. \& R.R. Newbold (2000). Potential endocrine-modulating effects of various phytoestrogens in the diet. Nutrition 16(7):658-662. https://doi.org/10.1016/So8999007(00)00306-3

Jeon, H.Y., Seo, D.B., Shin, H.-J. \& S.-J. Lee (2012). Effect of Aspergillus oryzae-challenged germination on soybean isoflavone content and antioxidant activity. Journal of Agricultural and Food Chemistry 60:2807-2814. https://doi.org/10.1021/jf204708n

Jerkovic, V. \& S. Collin (2007). Occurrence of resveratrol and piceid in American and European hop cones. Journal of Agricultural and Food Chemistry 55(21):8754-8758.

https://doi.org/10.1021/jfo71792k, http://www.ncbi.nlm.nih.gov/pubmed/17883256

Jerkovic, V. \& S. Collin (2008). Fate of resveratrol and piceid through different hop processings and storage times. Journal of Agricultural and Food Chemistry 56(2):584-590. https://doi.org/10.1021/jfo72465f, http://www.ncbi.nlm.nih.gov/pubmed/18163557

Jerkovic, V., D. Callemien \& S. Collin (2005). Determination of stilbenes in hop pellets from different cultivars. Journal of Agricultural and Food Chemistry 53(10):4202-4206. https://doi.org/10.1021/jfo50222m, http://www.ncbi.nlm.nih.gov/pubmed/15884861

Jerkovic, V., F. Nguyen, A. Timmermans \& S. Collin (2008). Comparison of procedures for resveratrol analysis in beer: Assessment of stilbenoids stability through wort fermentation and beer aging. Journal of the Institute for Brewing 114(2):143-149. https://doi.org/10.1002/j.20500416.2008.tboo319.x 
Ji, M., Li, Q., Ji, H. \& H. Lou (2014). Investigation of the distribution and season regularity of resveratrol in Vitis amurensis via HPLC-DAD-MS/MS. Food Chemistry 142:61-65.

https://doi.org/10.1016/j.foodchem.2013.06.131,

http://www.ncbi.nlm.nih.gov/pubmed/24001812

Joffee, A.Z. \& B. Shiray (1966). Soil and kernel mycoflora of groundnut fields in Israel. Mycologia 58:629-640. https://doi.org/10.2307/3757043

Johnsson P., A. Kamal-Eldin, L.N. Lundgren \& P. Åman (2000). HPLC method for analysis of secoisolariciresinol diglucoside in flaxseeds. Journal of Agricultural and Food Chemistry 48(11):5216-5219. https://doi.org/10.1021/jfooo5871

Jung, W., Yu, O., Lau, S.-M.C., O'Keefe, D.P., Odell, J., Fader, G. \& B. McGonigle (2000) Identification and expression of isoflavone synthase, the key enzyme for biosynthesis of isoflavones in legumes. Nature Biotechnology 18:208-212. https://doi.org/10.1038/72671

Kallela, K., K. Heinonen \& H. Saloniemi (1984). Plant oestrogens; the cause of decreased fertility in cows. Nordisk veterinärmedicin 36(3-4):124-129.

Kallithraka, S., I. Arvanitoyannis, A. El-Zajouli \& P. Kefalas (2001). The application of an improved method for trans-resveratrol to determine the origin of Greek red wines. Food Chemistry 75:355-363. https://doi.org/10.1016/S0308-8146(01)00213-8

Kanno, S., S. Hirano \& F. Kayama (2004). Effects of the phytoestrogen coumestrol on RANKligand-induced differantiation of osteoclasts. Toxicology 203:211-220. https://doi.org/10.1016/j.tox.2004.06.015

Kanno, J., L. Onyon, S. Peddada, J. Ashby, E. Jacob \& W. Owens (2003a). The OECD program to validate the rat uterotrophic bioassay. Phase 2: coded single-dose studies. Environmental Health Perspectives 11(12):1550-1558. https://doi.org/10.1289/ehp.5870

Kanno, J., L. Onyon, S. Peddada, J. Ashby, E. Jacob \& W. Owens (2003b). The OECD program to validate the rat uterotrophic bioassay. Phase 2: dose-response studies. Environmental Health Perspectives 11(12):1530-1549. https://doi.org/10.1289/ehp.5780

Kao, T.H.\& B.H. Chen (2002). An improved method for determination of isoflavones in soybean powder by liquid chromatography. Chromatographia 56:423-430. https://doi.org/10.1007/BFo2492004

Kape, R., M. Parniske \& D. Werner (1991). Chemotaxis and nod gene activity of Bradyrhizobium japonicum in response to hydroxycinnamic acids and isoflavonoids. Appliend and Environmental Microbiology 57(1):316-319.

Kariyone, T., M. Takahashi, T. Ito \& K. Masutani (1958). Chemical constituents of the plants of coniferae and allied orders. XXX. Studies on the component of the leaves of Picea glehnii Masters. (3) On the occurrence of picein and resveratrol. Yakugaku Zasshi 79:394-395. https://doi.org/10.1248/yakushi1947-79.3_394

Kariyone, T., M. Takahashi, T. Ito \& K. Masutani (1959). Chemical constituents of the plants of coniferae and allied orders. XXXII. Studies on the existence of resveratrol and 3,4dihydroxyacetophenone in the leaves of the plants of Picea. Yakugaku Zasshi 80:394-395. https://doi.org/10.1248/yakushi1947.79.3_394

Kataliníc, V., S.S. Možina, D. Skroza, I. Generalíc, H. Abramovíc, M. Miloš, I. Ljubenkov, S. Piskernik, I. Pezo, P. Terpinc \& M. Boban (2010). Polyphenolic profile, antioxidant properties and antimicrobial activity of grape skin extracts of 14 Vitis vinifera varieties grown in Dalmatia (Croatia). Food Chemistry 119:715-723. https://doi.org/10.1016/j.foodchem.2009.07.019

Katao, Y., H. Sawai, K. Inami, N. Domae \& N. Matsumoto (2011). Direct effects of estrogen on differentiation and apoptosis of osteclasts. Orthodontic Waves 70(1): 8-14. https://doi.org/10.1016/j.odw.2010.07.002

Kaufman, P.B., Duke, J.A., Brielmann, H., Boik, J. \& J.E. Hoyt (1997). A comparative survey of leguminous plants as sources of the isoflavones, genistein and daidzein: implications for human nutrition and health. The Journal of Alternative and Complementary Medicine 3(1):7-12. https://doi.org/10.1089/acm.1997.3.7 
Keen, N.T. (1975). The isolation of phytoalexins from germinating seeds of Cicer arietinum, Vigna sinensis, Arachis hypogaea, and other plants. Phytopathological Notes 65:91-92. https://doi.org/10.1094/Phyto-65-91

Keen, N.T. \& Ingham, J.L. (1976). New stilbene phytoalexins from American cultivars of Arachis hypogaea. Phytochemistry 15:1794-1795. https://doi.org/10.1016/So031-9422(00)97495-8

Keller, M., C.C. Steel \& G.L, Creasy (2000). Stilbene accumulation in grapevine tissues: Developmental an environmental effects. Acta Horticulturae 514:275-286. https://doi.org/10.1766o/ActaHortic.2000.514.33

Kim, J.-S., Hwang, Y.-H. \& H.-S. Lee (2003). Analysis of isoflavones for 66 varieties of sprout beans and bean sprouts. Korean Journal of Food Science and Technology 35(4):568-575.

Kim, J.-S., Kim, J.-G. \& W.-J. Kim (2004). Changes in isoflavone and oligosaccharides of soybeans during germination. Korean Journal of Food Science and Technology 36(2):294-298.

Kim, E.H., Kim, S.H., Chung, J.I., Chi, H.Y., Kim, J.A. \& I.M. Chung (2006). Analysis of phenolic compounds and isoflavones in soybean seeds (Glycine max (L.) Merill) and sprouts grown under different conditions. Eur Food Res Technol 222:201-208. https://doi.org/10.1007/s00217005-0153-4

Kim, E.-H., Ro, H.-M., Kim, S.-L., Kim, H.-S. \& I-M. Chung (2012). Analysis of isoflavone, phenolic, soyasapogenol, and tocopherol compounds in soybean [Glycine max (L.) Merrill] germplasms of different seed weights and origins. Journal of Agricultural and Food Chemistry 6o(23):6045-6055. https://doi.org/10.1021/jf300463f

Kim, J.K., E.H. Kim, O.K. Lee, S.Y. Park, B. Lee, S.H. Kim, I. Park \& I.M. Chung (2013). Variation and correlation analysis of phenolic compounds in mungbean (Vigna radiata $\mathrm{L}$.) varieties. Food Chemistry 141(3):2988-2997. https://doi.org/10.1016/j.foodchem.2013.05.060, http://www.ncbi.nlm.nih.gov/pubmed/23871050

Kimura, Y., H. Okuda \& M. Kubo (1995). Effects of stilbenes isolated from medicinal plants on arachidonate metabolism and degranulation in human polymorphonuclear leukocytes. Journal of Ethnopharmacology 45:131-139. https://doi.org/10.1016/0378-8741(94)01206-F

King A. \& G. Young (1999). Characteristics and occurrence of phenolic phytochemicals. Journal of the American Dietetic Association 99(2):213-218. https://doi.org/10.1016/Sooo28223(99)00051-6

King, F.E:, T,J, King, D.H. Godson \& L.C. Manning (1956). Chemistry of extractives from hardwood. XXVII. The occurrence of 3,3, 4, $5^{\prime}$-tetrahydroxy and 3,3',4,5,5'pentahydroxystilbene in Vouacapoua species. Journal of the Chemical Society, p. 4477-4480. https://doi.org/10.1039/JR9560004477

Király-Véghely, Z., E. Tyihák, L. Albert, Z.I. Németh \& G. Kátay (1998). Identification and measurement of resveratrol and formaldehyde in parts of white and blue grape berries. Acta Biologia Hungarica 49(2-4):281-289. http://www.ncbi.nlm.nih.gov/pubmed/10526971

Kitta, K. (2013). Availability and utility of crop composition data. Journal of Agricultural and Food Chemistry 61(35):8304-11. https://doi.org/10.1021/jf400777V

Klump, S.P., Allred, M.C., MacDonald, J.L. \& J.M. Ballam (2001). Determination of isoflavones in soy and selected foods containing soy by extraction, saponification, and liquid chromatography: collaborative study. Food Composition and Additives 84(6):1865-1883.

Knuckles, B.E., deFremery, D. \& G.O. Kohler (1976). Coumestrol content of fractions obtained during wet processing of alfalfa. Journal of Agricultural and Food Chemistry 24(6):1177-1180. https://doi.org/10.1021/jf60208a034

Kolouchová-Hanzlíková, I., K. Melzoch, V. Filip \& J. Smidrkal (2004). Rapid method for resveratrol determination by HPLC with electrochemical and UV detections in wines. Food Chemistry 87:151-158. https://doi.org/10.1016/j.foodchem.2004.01.028

Konar, N. (2013). Non-isoflavone phytoestrogenic compound contents of various legumes. European Food Research and Technology 236(3):523-530.

https://doi.org/10.1016/j.jfca.2011.11.004 
Konar, N., E.S. Poyrazoglu, K. Demir \& N. Artik (2012). Determination of conjugated and free isoflavones in some legumes by LC-MS/MS. Journal of Food Composition and Analysis 25(2):173-178. https://doi.org/10.1016/j.jfca.2011.11.004

Korhammer, S., F. Reniero \& F. Mattivi (1995). An oligostilbene from Vitis roots. Phytochemistry 38(6):1501-1504. https://doi.org/10.1016/0031-9422(94)00811-7

Kosslak, R.M., Bookland, R., Barkei, J., Paaren, H.E. \& E.R. Appelbaum (1987). Induction of Bradyrhizobium japonicum common nod genes by isoflavones isolated from Glycine max. Proceedings of the National Academy of Sciences USA 84:7428-7432. https://doi.org/10.1073/pnas.84.21.7428

Kostadinović, S., A. Wilkens, M. Stefova, V. Ivanova, B. Vojnoski, H. Mirhosseini \& P. Winterhalter (2012). Stilbene levels and antioxidant activity of Vranec and Merlot wines from Macedonia: effect of variety and enological practices.

http://www.ncbi.nlm.nih.gov/pubmed/22980903

Krasnow, M.N. \& T.M. Murphy (2004). Polyphenol glucosylating activity in cell suspensions of grape (Vitis vinifera). Journal of Agricultural and Food Chemistry 52:3467-3472. https://doi.org/10.1021/jfo35234r

Kraushofer T. \& G. Sontag (2002). Determination of matairesinol in flax seed by HPLC with coulometric electrode array detection. Journal of Chromatography B 777(1-2):61-66. https://doi.org/10.1016/S1570-0232(01)00577-3

Kudou, S., Fleury, Y., Welti, D., Magnolato, D., Uchida, T., Kitamura, K. \& K. Okubo, (1991). Malonyl isoflavone glycosides in soybean seeds (Glycine max Merrill). Agricultural and Biological Chemistry 55(9):2227-2233. https://doi.org/10.1271/bbb1961.55.2227

Kuhnle, G.C., C. Dell'Aquila, Y-L. Low, M. Kussmaul \& S.A. Bingham (2007). Extraction and quantification of phytoestrogens in foods using automated solid-phase extraction and LC/MS/MS. Analytical chemistry 79(23):9234-9239. https://doi.org/10.1021/ac701732r

Kuhnle, G., C. Dell'Aquila, S.M. Aspinall, S.A. Runswick, A. Mulligan \& S.A. Bingham (2008a). Phytoestrogen content of foods of animal origin: dairy products, eggs, meat, fish, and seafood. Journal of Agricultural and Food Chemistry 56(21):10099-10104. https://doi.org/10.1021/jf801344X

Kuhnle, G., C. Dell'Aquila, S.M. Aspinall, S.A. Runswick, A. Mulligan \& S.A. Bingham (2008b). Phytoestrogen content of beverages, nuts, seeds, and oils. Journal of Agricultural and Food Chemistry 56(16):7311-7315. https://doi.org/10.1021/jf8015349

Kuhnle, G., C. Dell'Aquila, S.M. Aspinall \& S.A. Runswick (2009a). Phytoestrogen content of cereals and cereal-based foods consumed in the UK. Nutrition and Cancer 61(3):302-309. https://doi.org/10.1080/01635580802567141

Kuhnle, G., C. Dell'Aquila, S.A. Runswick \& S.A. Bingham (200gb). Variability of phytoestrogen content in foods from different sources. Food Chemistry 113(4):1184-1187. https://doi.org/10.1016/j.foodchem.2008.08.004

Kuiper, G.C., B. Carlsson, K. Grandien, E. Enmark, J. Häggblad, S. Nilsson \& J-Å. Gustafsson (1997). Comparison of the ligand binding specificity and transcript tissue distribution of estrogen receptors $\alpha$ and $\beta$. Endocrinology 138(3):863-870. https://doi.org/10.1210/endo.138.3.4979

Kuiper, G., J. Lemmen, B. Carlsson, C. Corton, S. Safe, P. van der Saag, B. van der Burgh \& J-Å. Gustafsson (1998). Interaction of estrogenic chemicals and phytoestrogens with estrogen receptor $\beta$. Endocrinology 139(10):4252-4263. https://doi.org/10.1210/endo.139.10.6216

Kumar, V., A. Rani, A.K. Dixit, D. Pratap \& D. Bhatnagar (2010) A comparative assessment of total phenolic content, ferric reducing-anti-oxidative power, free radical scafenging activity, vitamin $\mathrm{C}$ and isoflavones content in soybean with varying seed coat colour. Food Research International 43:323-328. https://doi.org/10.1016/j.foodres.2009.10.019

Lambert, C., T. Richard, E. Renouf, J. Bisson, P. Waffo-Téguo, L. Bordenave, N. Ollat, J.M. Mérillon \& S. Cluzet (2013). Comparative analyses of stilbenoids in canes of major Vitis vinifera L. cultivars. Journal of Agricultural and Food Chemistry 61(47):11392-11399.

https://doi.org/10.1021/jf403716y, http://www.ncbi.nlm.nih.gov/pubmed/24171397 
Lamikanra, O., C.C. Grimm, J.B. Rodin \& I.D. Inyang (1996). Hydroxylated stilbenes in selected American wines. Journal of Agricultural and Food Chemistry 44:1111-1115.

https://doi.org/10.1021/jf950274j

Lamuela-Raventós, R.M. \& A.L. Waterhouse (1993). Occurrence of resveratrol in selected California wines by a new HPLC method. Journal of Agricultural and Food Chemistry 41(4):521523. https://doi.org/10.1021/jfooo28aoo1

Lamuela-Raventós, R.M., A.I. Romero-Pérez, A.L. Waterhouse \& M.C. de la Torre-Boronat (1995). Direct HPLC analysis of cis- and trans-resveratrol and piceid isomers in Spanish red Vitis vinifera wines. Journal of Agricultural and Food Chemistry 43:281-283. https://doi.org/10.1021/jfooo50a003

Langcake, P. (1981) Disease resistance of Vitis spp. And the production of the stress metabolites resveratrol, $\varepsilon$-viniferin, $\alpha$-viniferin and pterostilbene. Physiological Plant Pathology 18:213-226. https://doi.org/10.1016/S0048-4059(81)80043-4

Langcake, P. \& W.V. McCarthy (1979). The relationship of resveratrol production to infection of grapevine leaves by Botrytis cinerea. Vitis 18:244-253.

Langcake, P. \& R.J. Pryce (1976). The production of resveratrol by Vitis vinifera and other members of the Vitaceae as a response to infection or injury. Physiological Plant Pathology 9:77-86. https://doi.org/10.1016/0048-4059(76)90077-1

Langcake, P. \& R.J. Pryce (1977a). The production of resveratrol and the viniferins by grapevines in response to ultraviolet irradiation. Phytochemistry 16:1193-1196.

https://doi.org/10.1016/S0031-9422(00)94358-9

Langcake, P. \& R.J. Pryce (1977b). A new class of phytoalexins from grapevines. Experientia 33:151-152. https://doi.org/10.1007/BF02124034

Larronde, F., J.P. Gaudillère, S. Krisa, A. Decendit, G. Deffieux \& J.M. Mèrillon (2003). Airborne methyl jasmonate induces stilbene accumulation in leaves and berries of grapevine plants. American Journal of Enology and Viticulture 54(1):63-66.

La Torre, G.L., G. Laganà, E. Bellocco, F. Vilasi, F. Salvo \& G. Dugo (2004). Improvement on enzymatic hydrolysis of resveratrol glucosides in wine. Food Chemistry 85:259-266. https://doi.org/10.1016/j.foodchem.2003.06.019

Leblanc, M.R., C.E. Johnson \& P.W. Wilson (2008). Influence of pressing method on juice stilbene content in muscadine and bunch grapes. Journal of Food Science 73(4): $\mathrm{H}_{5} 8-62$. https://doi.org/10.1111/j.1750-3841.2008.00733.x

Lee, J. \& C. Rennaker (2007) Antioxidant capacity and stilbene contents of wines produced in the Snake River Valley of Idaho. Food Chemistry 105:195-203.

https://doi.org/10.1016/j.foodchem.2007.03.069

Lee, H.P., L. Gourley, S.W: Duffy, J. Esteve, J. Lee \& N.E. Day (1991). Dietary effects on breastcancer risk in Singapore. Lancet 337:1197-1200. https://doi.org/10.1016/0140-6736(91)92867-2

Lee, M.-h., Park, Y.-H., Oh, H.-s. \& T.-S. Kwak (2002). Isoflavone content in soybean and its processed products. Korean Journal of Food Science and Technology 34(3):568-575.

Lee, S.J., Yan, W., Ahn, J.K. \& I.M. Chung (2003a). Effects of year, site, genotype and their interactions on various soybean isoflavones. Fieald Crops Research 81(2):181-192. https://doi.org/10.1016/S0378-4290(02)00220-4

Lee, S.J., Ahn, J.K., Kim, S.H., Kim, J.T., Han, S.J., Jung, M.Y. \& I.M. Chung (2003b). Variation in isoflavone of soybean cultivars with location and storage duration. Journal of Agricultural and Food Chemistry 51:3382-3389. https://doi.org/10.1021/jfo261405

Lee, J. M., Renita, R.J., Fioritto, S.K. St. Martin, S.J. Schwartz \& Y. Vodovotz (2004). Isoflavone characterization and antioxidant activity of Ohio soybeans. Journal of Agricultural and Food Chemistry 52:2647-2651. https://doi.org/10.1021/jfo35426m

Lee, H.-y., Kim, J.-s., Kim, Y.-S. \& W.-J. Kim (2005). Isoflavone and quality improvement of soymilk by using germinated soybean. Korean Journal of Food Science and Technology $37(3): 443-448$. 
Lee, S.-J., Ahn, J-K., Khanh, T-D., Chun, S-C., Kim, S-L., Ro, H-M., Song, H-K. \& I-M. Chung (2007). Comparison of isoflavone concentrations in soybean (Glycine max (L.) Merrill) sprouts grown under two different light conditions. Journal of Agricultural and Food Chemistry 55(23):9415-9421. https://doi.org/10.1021/jfo71861v

Lee, J.H., Jeong, S.W., Cho, Y.A., Park, S., Kim, Y.-H., Bae, D.W., Chung, J.I., Kwak, Y.-S., Jeong, M.-J., Park, S.-C., Shim, J.-H., Jin, J.S. \& S.C. Shin (2013). Determination of the variations in levels of phenolic compounds in soybean (Glycine max Merr.) sprouts infected by anthracnose (Colletrotrichum gloeosporioides). Journal of the Science of Food and Agriculture 93:3081-3086. https://doi.org/10.1002/jsfa.6142

Leopold, S., M. Erwin, J. Oh \& B. Browning (1976). Phytoestrogens: adverse effects on reproduction in California quail. Science 191(4222):98-100.

https://doi.org/10.1126/science.1246602

Lepping, M.D., R.A. Herman \& B.L. Potts (2013). Compositional equivalence of DAS-444Ø6-6 (AAD-12 + 2mEPSPS + PAT) herbicide-tolerant soybean and nontransgenic soybean. Journal of Agricultural and Food Chemistry 61(46):11180-1119o. https://doi.org/10.1021/jf403775d

Leuner, O., J. Havlik, J. Hummelova, E. Prokudina, P. Novy \& L. Kokoska (2013). Distribution of isoflavones and coumestrol in neglected tropical and subtropical legumes. Journal of the Science of Food and Agriculture 93:575-579. https://doi.org/10.1002/jsfa.5835

Li, X., B. Wu, L. Wang \& S. Li (2006). Extractable amounts of trans-resveratrol in seed and berry skin in Vitis evaluated at the germplasm level. Journal of Agricultural and Food Chemistry 54:8804-8811. https://doi.org/10.1021/jfo61722y

Li, Z., Q. Pan, Z. Jin, L. Mu \& C. Duan (2011). Comparison of phenolic compounds in Vitis vinifera cv. Cabernet Sauvignon wines from vie wine-growing regions in China. Food Chemistry 125:7783. https://doi.org/10.1016/j.foodchem.2010.08.039

Liggins, J., Bluck, L.J.C., Runswick, S., Atkinson, C., Coward, W.A. \& S.A. Bingham (2000a). Daidzein and genistein content of fruits and nuts. The Journal of Nutritional Biochemistry 11(6)326-331. https://doi.org/10.1016/So955-2863(00)00085-1

Liggins, J., Bluck, L.J.C., Runswick, S., Atkinson, C., Coward, W.A. \& S.A. Bingham (200ob). Daidzein and genistein contents of vegetables. British Journal of Nutrition 84(5):717-725.

Liggins J., R. Grimwood \& S.A. Bingham (2000c). Extraction and quantification of lignan phytoestrogens in food and human samples. Analytical Biochemistry 287(1):102-109. https://doi.org/10.1006/abio.2000.4811

Liggins, J., Mulligan, A., Runswick, S. \& S.A. Bingham (2002). Daidzein and genistein content of cereals. European Journal of Clinical Nutrition 56(10):961-966.

https://doi.org/10.1038/sj.ejcn.1601419

Lima M dos, S., S. Silani Ide, I.M. Toaldo, L.C. Corrêa, A.C. Biasoto, G.E. Pereira, M.T. Bordignon-Luiz \& J.L. Ninow (2014). Phenolic compounds, organic acids and antioxidant activity of grape juices produced from new Brazilian varieties planted in the Northeast Region of Brazil. Food Chemistry 161:94-103. https://doi.org/10.1016/j.foodchem.2014.03.109, http://www.ncbi.nlm.nih.gov/pubmed/24837926

Lin, F. \& M.M. Giusti (2005). Effects of solvent polarity and acidity on the extraction efficiency of isoflavones from soybeans (Glycine max). Journal of Agricultural and Food Chemistry 53(10):3795-3800. https://doi.org/10.1021/jf05003or

Lin, H., J. Rao, J. Shi, C. Hu, F. Cg, Z.A. Wilson, D. Zhang \& S. Quan (2014). A seed metabolomic study reveals significant metabolite variations and correlations among different soybean cultivars. Journal of Integrative Plant Biology 56(9):826-836. https://doi.org/10.1111/jipb.12228

Lindner, H.R. (1976). Occurrence of anabolic in plants and their importance. Environmental Quality and Safety Supplement 5:151-158.

Liswidowati, F., F. Melchior, F. Hohmann, B. Schwer \& H. Kindl (1991). Induction of stilbene synthase by Botrytis cinerea in cultured grapevine cells. Planta 183:307-314. https://doi.org/10.1007/BFo0197803

Liu, K. (1997). Fermented oriental soyfoods. In Soybeans: Chemistry, Technology, and Utilization, Chapman and Hall: New York, pp. 218-296. https://doi.org/10.1007/978-1-4615-1763-4_5 
Liu, K.S. (2004). Soybeans as a powerhouse of nutrients and phyhtochemicals. Editor. American Oil Chemists' Society, Champaign, Illinoi.

Liv, R., Hu, Y., Li, J. \& Z. Lin (2007). Production of soybean isoflavone genistein in non-legume plants via genetically modified secondary metabolism pathway. Metabolic Engineering 9:1-7. https://doi.org/10.1016/j.ymben.2006.08.003

Liu, C., L. Wang, J. Wang, B. Wu, W. Liu, P. Fan, Z. Liang \& S. Li (2013). Resveratrols in Vitis berry skins and leaves: their extraction and analysis by HPLC. Food Chemistry 136(2):643-649. https://doi.org/10.1016/j.foodchem.2012.08.017, http://www.ncbi.nlm.nih.gov/pubmed/23122109

Liukkonen, K.-H., K. Katina, A. Wilhelmsson, O. Myllymäki, A.-M. Lampi, S. Kariluoto, V. Piironen, S.-M. Heinonen, T. Nurmi, H. Adlercreutz, A. Peltoketo, J.-M. Pihlava, V. Hietaniemi $\&$ K. Poutanen (2003). Process-induced changes on bioactive compounds in whole grain rye. Proceedings of the Nutrition Society 62:117-122. https://doi.org/10.1079/PNS2002218

Loizzo, M.R., S. Nigro, D. De Luca \& F. Menichini (2011). Detection of ochratoxin A and cis- and trans-resveratrol in red wines and their musts from Calabria (Italy). Food Additives and Contaminants Part A Chemistry, Analysis, Control, Exposure and Risk Assessment 28(11):15611568. https://doi.org/10.1080/19440049.2011.590454, http://www.ncbi.nlm.nih.gov/pubmed/21732871

López, M., F. Martinez, C. Del Valle, C. Orte \& M. Miró (2001). Analysis of phenolic constituents of biological interest in red wines by high-performance liquid chromatography. Journal of Chromatography A 922:359-363. https://doi.org/10.1016/So021-9673(01)00913-X

López-Alfaro, I., L. González-Arenzana, N. López, P. Santamaría, R. López \& T. Garde-Cerdán (2013). Pulsed electric field treatment enhanced stilbene content in Graciano, Tempranillo and Grenache grape varieties. Food Chemistry 141(4):3759-3765.

https://doi.org/10.1016/j.foodchem.2013.06.082,

http://www.ncbi.nlm.nih.gov/pubmed/23993546

Lozovaya, V.V., A.V. Lygin, A.V. Ulanov, R.L. Nelson, J. Daydé \& J.M. Widholm (2005). Effect of temperature and soil moisture status during seed development on soybean seed isoflavone concentration and composition. Crop Science 45:1934-1940.

https://doi.org/10.2135/cropsci2004.0567

Luan, T., G. Li \& Z. Zhang (2000). Gas-phase postderivatization following solid-phase microextraction for rapid determination of trans-resveratrol in wine by gas chromatographymass spectrometry. Analytica Chimica Acta 424:19-25. https://doi.org/10.1016/So0032670(00)01103-X

Lucena, A.P.S., R.J.B. Nascimento, J.A.C. Maciel, J.X. Tavares, J.M. Barbosa-Filho \& E.J. Oliveira (2010). Antioxidant activity and phenolics content of selected Brazilian wines. Journal of Food Composition and Analysis 23:30-36. https://doi.org/10.1016/j.jfca.2009.08.004

Luh, B.S. (1995). Industrial production of soy sauce. Journal of Industrial Microbiology 14:467471. https://doi.org/10.1007/BF01573959

Lundry, D.R., W.P. Ridley, J.J. Meyer, S.G. Riordan, M.A. Nemeth, W.A. Trujillo, M.L. Breeze \& R. Sorbet (2008). Composition of grain, forage, and processed fractions from secondgeneration glyphosate-tolerant soybean, MON 89788, is equivalent to that of conventional soybean (Glycine max L.). Journal of Agricultural and Food Chemistry 56(12):4611-22. https://doi.org/10.1021/jfo73087h

Lyons, M.M., C. Yu, R.B. Toma, S.Y. Cho, W. Reiboldt, J. Lee \& R.B. van Breemen (2003). Resveratrol in raw and baked blueberries and bilberries. Journal of Agricultural and Food Chemistry 51(20):5867-5870. https://doi.org/10.1021/jfo3415of, http://www.ncbi.nlm.nih.gov/pubmed/13129286

Malenčić, D., Cvejić, J. \& J. Miladinović (2012). Polyphenol content and antioxidant properties of coored soybean seeds from central Europe. Journal of Medicinal Food 15(1):89-95.

https://doi.org/10.1089/jmf.2010.0329 
Malovaná, S., F.J. Garcia Montelongo, J.P. Pérez \& M.A. Rodriguez-Delgado (2001) Optimisation of sample preparation for the determination of trans-resveratrol and other polyphenolic compounds in wines by high performance liquid chromatography. Analytica Chimica Acta 428:245-253. https://doi.org/10.1016/So003-2670(00)01231-9

Manach, C.A., Scalbert, A., Morand, C., Remesy, C. \& L. Jimenez (2004). Polyphenols: Food sources and bioavailability. American Journal of Clinical Nutrition 79:727-747.

Mark, L., M.S. Pour Nikfardjam, P. Avar \& R. Ohmacht (2005). A validated HPLC method for the quantitative analysis of trans-resveratrol and trans-piceid in Hungarian wines. Journal of Chromatographic Science 43:445-449. https://doi.org/10.1093/chromsci/43.9.445

Mathias, K., Ismail, B., Corvalan, C.M. \& K.D. Hayes (2006). Heat and pH effects on the conjugated forms of genistin and daidzin isoflavones. Journal of Agricultural and Food Chemistry 54:7495-7502. https://doi.org/10.1021/jfo61322a

Matsuara, M., A. Obata \& D. Fukushima (1989). Objectionable flavour of soy milk developed during the soaking of soybeans and its control. Journal of Food Science 54(3):602-605. https://doi.org/10.1111/j.1365-2621.1989.tbo4662.x

Mattivi, F. (1993a). Solid phase extraction of trans-resveratrol from wines for HPLC analysis. Zeitschrift für Lebensmittel-Untersuchung und Forschung 196:522-525. https://doi.org/10.1007/BF01201331

Mattivi, F. (1993b). Il contenuto di resveratrolo nei vini rossi e rosati trentini del commercio. Rivista di Viticoltura e di Enol.ogia 46(1):37-45.

Mattivi, F. \& G. Nicolini (1993). Influenza della tecnica di vinificazione sul ontenuto di resveratrolo dei vini. L'Enotecnico Luglio/Agosto 1993:81-88.

Mattivi, F., F. Reniero \& S. Korhammer (1995). Isolation, characterization, and evolution in red wine vinification of resveratrol monomers. Journal of Agricultural and Food Chemistry 43:18201823. https://doi.org/10.1021/jfooo55a013

Mazur, W. (1998) Phytoestrogen content in foods. In: Baillièr's Clinical Endocrinology and Metabolism, Volume 12(4):729-742. https://doi.org/10.1016/So950-351X(98)80013-X

Mazur, W. \& H. Adlercreutz (1998). Natural and anthropogenic environmental oestrogens: the scientific basis for risk assessment. Naturally occurring oestrogens in food. Pure \% ' \& Applied Chemistry 70(9):1759-1776. https://doi.org/10.1351/pac199870091759

Mazur W., T. Fotsis, K. Wähälä, S. Ojala, A. Salakka \& H. Adlercreutz (1996). Isotope dilution gas chromatographic-mass spectrometric method for the determination of isoflavonoids, coumestrol, and lignans in food samples. Analytical Biochemistry 233(2):169-180. https://doi.org/10.1006/abio.1996.0025

Mazur W.M., K. Wähälä, S. Rasku, A. Salakka, T. Hase \& H. Adlercreutz (1998a). Lignans and isoflavonoid concentrations in tea and coffee. British Journal of Nutrition 79(1):37-45. https://doi.org/10.1079/BJN19980007

Mazur W.M., J.A. Duke, K. Wähäälä, S. Rasku \& H. Adlercreutz (1998b). Isoflavonoids and lignans in legumes: nutritional and health aspects in humans. Nutritional Biochemistry 9(4):193-200. https://doi.org/10.1016/So955-2863(97)00184-8

McCann, M.C., K. Liu, W.A. Trujillo \& R.C. Dobert (2005). Glyphosate-tolerant soybeans remain compositionally equivalent to conventional soybeans (Glycine max L.) during three years of field testing. Journal of Agricultural and Food Chemistry 53(13):5331-5335. https://doi.org/10.1021/jf0504317

McMurtrey, K.D., J. Minn, K. Pobanz \& T.P. Schultz (1994). Analysis of wines for resveratrol using direct injection high-pressure liquid chromatography with electrochemical detection. Journal of Agricultural and Food Chemistry 42(10):2077-2080. https://doi.org/10.1021/jfooo46aoo1

Mebrahtu T., A. Mohamed, C.Y. Wang \& T. Andersen (2004). Analysis of isoflavone contents in vegetable soybeans. Plant Foods for Human Nutrition 59(2):55-61.

https://doi.org/10.1007/s11130-004-0023-4 
Meksem, K., Njiti, V.N., Banz, W.J., Iqbal, M.J., Kassem, M.M., Hyten, D.L., Yuang, J., Winters, T.A. \& D.A. Lightfoot (2001). Genomic regions that underlie soybean seed isoflavone content. Journal of Biomedicine and Biotechnology 1(1):38-44. https://doi.org/10.1155/S1110724301000110

Melzoch, K., I. Hanzlikova, V. Filip, D. Buckiova \& J. Smidrkal (2001). Resveratrol in parts of vine and wine originating from Bohemian and Moravian vineyard regions. Agriculturae Conspectus Scientificus 66:53-57.

Metzger, D., M. Berry, S. Ali \& P. Chamber (1995). Effect of antagonists on DNA binding properties of the human estrogen receptor in vitro and in vivo. Molecular Endocrinology 9:579591.

Miksicek, R.J. (1995). Estrogenic flavonoids: structural requirements for biological activity. Proceedings of the Society of Experimental Biology and Medicine 208:44-50. https://doi.org/10.3181/00379727-208-43830

Milder, I.E.J., I.C.W. Arts, B. van de Putte, D.P. Venema \& P.C.H. Hollman (2005). Lignan contents of Dutch plant foods: a database including lariciresinol, pinoresinol, secoisolariciresinol and matairesinol. British Journal of Nutrition 93(3):393-402. https://doi.org/10.1079/BJN20051371

Mo, H., S. Kariluoto, V. Piironen, Y. Zhu, M.G. Sanders, J.-P. Vincken, J. Wolkers-Rooijaackers \& M.J.R. Nout (2013). Effect of soybean processing on content and bioaccessibility of folate vitamin B12 and isoflavones in tofy and tempe. Food Chemistry 141:2418-2425. https://doi.org/10.1016/j.foodchem.2013.05.017

Mohanty, B., S.M. Basha, D.W. Gorbet, R.J. Cole \& J.W. Dorner (1991). Variation in phytoalexin production by peanut seed from several genotypes. Peanut Science 18:19-22.

https://doi.org/10.3146/ioog5-3679-18-1-7

Monagas, M., B. Bartolomé \& C. Fómez-Cordovés (2005). Evolution of polyphenols in red wines from Vitis vinifera L. during aging in the bottle. II. Non-anthocyanin phenolic compounds. European Journal of Food Research and Technology 220:331-340. https://doi.org/10.1007/s00217-004-1109-9

Moreno-Labanda, J.F., R. Mallavia, L. Pérez-Fons, V. Lizama, D. Saura \& V. Micol (2004) Determination of piceid and resveratrol in Spanish wines deriving from Monastrell (Vitis vinifera L.) grape variety. Journal of Agricultural and Food Chemistry 52(17):5396-5403. https://doi.org/10.1021/jfo49521m, http://www.ncbi.nlm.nih.gov/pubmed/15315376

Morito, K., T. Hirose, J. Kinjo, T. Hirikawa, M. Okawa, T. Nohara, S. Ogawa, S. Inove, M. Muramatsu \& Y. Masamune (2001). Interaction of phytoestrogens with estrogen receptor $\alpha$ and $\beta$. Biological \& Pharmaceutical Bulletin 24(4):351-356. https://doi.org/10.1248/bpb.24.351

Morris, P.F., Savard, M.E. \& E.W.B. Ward (1991). Identification and accumulation of isoflavonoids and isoflavone glucosides in soybean leaves and hypocotyls in resistance responses to Phytophthora megasperma f.sp. glycinea. Physiological and Molecular Plant Pathology 39:229-244. https://doi.org/10.1016/0885-5765(91)90006-4

Morrison, M.J., E.R. Cober, M.F. Saleem, N.B. McLaughlin, J.Frégeau-Reid, B.L. Ma, W. Yan \& L. Woodrow (2008). Changes in isoflavone concentration with 58 years of genetic improvement of short-season soybean cultivars in Canada. Crop Science 48:2201-2208. https://doi.org/10.1016/0885-5765(91)90006-4

Müllner, C. \& G. Sontag (1999). Determination of some phytoestrogens in soybeans and their processed products with HPLC and coulometric electrode array detection. Fresenius' Journal of Analytical Chemistry 364(3):261-265. https://doi.org/10.1007/s002160051334

Mueller, S., S. Simon, K. Chae, M. Metzler \& K. Korach (2004). Phytoestrogens and their human metabolites show distinct agonistic and antagonistic properties on estrogen receptor $\alpha(E R \alpha)$ and ER $\beta$ in human cells. Toxicological Sciences 80:14-25. https://doi.org/10.1093/toxsci/kfh147

Murkies, A.L., G. Wilcox \& S.R. Davis (1998). Phytoestrogens. Journal of Clinical Endocrinology and Metabolism 83:297-303. https://doi.org/10.1210/jc.83.2.297 
Natividade, M.M.P., L.C. Corrēa, S.V.C. de Souza, G.E. Pereira \& L.C. de O. Lima (2013). Simultaneous analysis of 25 phenolic compounds in grape juice for HPLC: Method validation and characterization of Sāo Francisco Valley samples. Microchemical Journal 110:665-674. https://doi.org/10.1016/j.microc.2013.08.010

Nurmi, T., S. Heinonen, W. Mazur, T. Deyama, S. Nishibe \& H. Adlercreutz (2003). Lignans in selected wines. Food Chemistry 83:303-309. https://doi.org/10.1016/S0308-8146(03)00222-X

Nevado, J.J.B., A.M.C. Salcedo \& G.C. Peňalvo (1999). Simultaneous determination of cis- and trans-resveratrol in wines by capillary zone electrophoresis. Analyst 124:61-66.

https://doi.org/10.1039/a807226d

Nikov, G., N. Hopkins, S. Boue \& W. Alworth (2000). Interactions of dietary estrogens with human estrogen receptors and the effect on estrogen receptor-estrogen response element complex formation. Environmental Health Perspectives 108(9):867-872.

https://doi.org/10.1289/ehp.00108867

Nilsson M., P. Åman, H. Härkönen, G. Hallmans, K.E.B. Knudsen, W. Mazur \& H. Adlercreutz (1997). Nutrient and lignan content, dough properties and baking performance of rye samples used in Scandinavia. Acta Agriculture Scandinavica, Section B, Soil and Plant Science 47(1):2634. https://doi.org/10.108o/ogo64719709362435

Nishikawa, J., K. Saito, J. Goto, F. Dakeyama, M. Matsuo \& T. Nishihara (1999). New screening methods for chemicals with hormonal activities using interaction of nuclear hprmone receptor with coactivator. Toxicology and Applied Pharmacology 154(1):76-83. https://doi.org/10.1006/taap.1998.8557

Nour, V., I. Trandafir \& C. Muntean (2012). Ultraviolet irradiation of trans-resveratrol and HPLC determination of trans-resveratrol and cis-resveratrol in Romanian red wines. Journal of Chromatographical Science 50(10):920-927. https://doi.org/10.1093/chromsci/bmso91, http://www.ncbi.nlm.nih.gov/pubmed/22689901

OECD (1983). One-generation reproduction toxicity study. OECD guideline for the testing of chemicals 415 .

OECD (2001). Two-generation reproduction toxicity study. OECD guideline for the testing of chemicals 416.

OECD (2007). Uterotrophic bioassay in rodents: a short-term screening test for oestrogenic properties. OECD guideline for the testing of chemicals 440.

OECD (2012a). Performance-based test guideline for stably transfected transactivation in vitro assays to detect estrogen receptor agonists. OECD guideline for the testing of chemicals 455 .

OECD (2012b). Revised Concensus Document on compositional considerations for new varieties of soybean [Glycine max (L.) Merr]. Key food and feed nutrients, anti-nutrients, toxicants and allergens. Series on the Safety of Novel Foods and Feeds, No 25, 48 pp.

Ogungbamila, F.O., G.O. Onawunmi, J.C. Ibewuike \& K.A. Funmilayo (1997). Antibacterial constituents of Ficus barteri fruits. International Journal of Pharmacognosy 35(3):185-189. https://doi.org/10.1076/phbi.35-3.185.13291

Okasaka, M., Y. Takaishi, K. Kogure, K. Fukuzawa, H. Shibata, T. Higuti, G. Honda, M. Ito, O.K. Kodzhimatov \& O. Shurmetov (2004). New stilbene derivatives from Calligonum leucocladum. Journal of Natural Products 67:1044-1046. https://doi.org/10.1021/np0304823

Okubo, K., lijima, M., Kobayashi, Y., Yoshikoshi, M., Uchida, T. \& S. Kudou (1992). Components responsible for the undesirable teste of soybean seeds. Bioscience, Biotechnology and Biochemistry 56(1):99-103. https://doi.org/10.1271/bbb.56.99

Oomah, B.D. (2002). Phytoestrogens. In: W.J. Hurst (Ed.). Methods of Analysis for Functional Foods and Nutraceuticals, CRC Press, pp.1-65. https://doi.org/10.1201/9781420014679.ch1

Orsini, F., F. Pelizzoni, L. Verotta \& T. Aburjai (1997). Isolation, synthesis, and antiplatelet aggregation activity of resveratrol $3-\mathrm{O}-\beta$-D-glucopyranoside and related compounds. Journal of Natural Products 60:1082-1087. https://doi.org/10.1021/np97006gt 
Padgette, S.R., K.H. Kolacz, X. Delannay, D.B. Re, B.J. LaVallee, C.N. Tinius, W.K. Rhodes, Y.I. Otero, G.F. Barry, D.A. Eichholtz, V.M. Peschke, D.L. Nida, N.B. Taylor \& G.M. Kishore (1995). Development, identification, and characterization of a glyphosate-tolerant soybean line. Crop Science 35:1451-1461. https://doi.org/10.2135/cropsci1995.0011183X003500050032X

Padgette, S.R., N.B. Taylor, D.L. Nida, M.R. Bailey, J. MacDonald, L.R. Holden \& R. L. Fuchs (1996). The composition of glyphosate-tolerant soybean seeds is equivalent to that of conventional soybeans. Journal of Nutrition 126(3):702-716.

Padilla, E., E. Ruiz, S. Redondo, A. Gordillo-Moscoso, K. Slowing \& T. Tejerina (2005). Relationship between vasodilation capacity and phenolic content of Spanish wines. European Journal of Pharmacology 517(1-2):84-91. https://doi.org/10.1016/j.ejphar.2005.04.044, http://www.ncbi.nlm.nih.gov/pubmed/15967426

Palomino, O., M.P. Gómez-Serranillos, K. Slowing, E. Carretero \& A. Villar (2000). Study of polyphenols in grape berries by reversed-phase high-performance liquid chromatography. Journal of Chromatography A 870(1-2):449-451. https://doi.org/10.1016/Soo21-9673(99)01225X, http://www.ncbi.nlm.nih.gov/pubmed/10722101

Pascual-Martí, M.C., A. Salvador, A. Chafer \& A. Berna (2001). Supercritical fluid extraction of resveratrol from grape skin of Vitis vinifera and determination by HPLC. Talanta 54(4):735740. https://doi.org/10.1016/S0039-9140(01)00319-8, http://www.ncbi.nlm.nih.gov/pubmed/18968296

Pastrana-Bonilla, E., C.C. Akoh, S. Sellappan \& G. Krewer (2003). Phenolic content and antioxidant capacity of muscadine grapes. Journal of Agricultural and Food Chemistry 51(18):5497-5503. https://doi.org/10.1016/S0039-9140(01)00319-8, http://www.ncbi.nlm.nih.gov/pubmed/12926904

Paucar-Menacho, L.M., M.A. Berhow, J.M.G. Mandarino, Y.K. Chang \& E.G. de Mejia (2010). Effect of time and temperature on bioactive compounds in germinated Brazilian soybean cultivar BRS 258. Food Research International 43:1856-1865.

https://doi.org/10.1016/j.foodres.2009.09.016

Paul, B., I. Masih, J. Deopujari \& C. Charpentier (1999). Occurrence of resveratrol and pterostilbene in age-old darakchasava, an eyurvedic medicine from India. Journal of Ethnopharmacology 68:71-76. https://doi.org/10.1016/S0378-8741(99)00044-6

Paulo, L., F. Domingues, J.A. Queiroz \& E. Gallardo (2011). Development and validation of an analytical method for the determination of trans- and cis-resveratrol in wine: analysis of its contents in 186 Portuguese red wines. Journal of Agricultural and Food Chemistry 59(6):21572168. https://doi.org/10.1021/jf105004y, http://www.ncbi.nlm.nih.gov/pubmed/21361293

Peňalvo J.L., S.M. Heinonen, T. Nurmi, T. Deyama, S. Nishibe \& H. Adlercreutz (2004). Plant lignans in soy-based health supplements. Journal of Agricultural and Food Chemistry 52(13):4133-4138. https://doi.org/10.1021/jfo497509

Peñalvo J.L., K.M. Haajanen, N. Botting \& H. Adlercreutz (2005). Quantification of lignans in food using isotope dilution gas chromatography/mass spectrometry. Journal of Agricultural and Food Chemistry 53(24):9342-9347. https://doi.org/10.1021/jfo51488w

Peñalvo J.L., H. Adlercreutz, M. Uehara, A. Ristimaki \& S. Watanabe (2008). Lignan content of selected foods from Japan. Journal of Agricultural and Food Chemistry 56(2):401-409.

https://doi.org/10.1021/jfo72695U

Pérez-Trujillo, J.P., Z. Hernández, F.J. López-Bellido \& I. Hermosín-Gutiérrez (2011). Characteristic phenolic composition of single-cultivar red wines of the Canary Islands (Spain). Journal of Agricultural and Food Chemistry 59(11):6150-6164. https://doi.org/10.1021/jf200881s, http://www.ncbi.nlm.nih.gov/pubmed/21548631

Perrone, G., I. Nicoletti, M. Pascale, A.D. Rossi, A.D. Girolamo \& A. Visconti (2007). Positive correlation between high levels of ochratoxin $A$ and resveratrol-related compounds in red wines. Journal of Agricultural and Food Chemistry 55(16):6807-6812.

https://doi.org/10.1021/jfo70993l, http://www.ncbi.nlm.nih.gov/pubmed/17625875

Peterson J., J. Dwyer, H. Adlercreutz, A. Scalbert, P. Jacques \& M.L. McCullough (2010). Dietary lignans: physiology and potential for cardiovascular disease risk reduction. Nutrition Reviews 68(10):571-603. https://doi.org/10.1111/j.1753-4887.2010.00319.x 
Pezet, R. \& P. Cuenat (1996). Resveratrol in wine: Extraction from skin during fermentation and post-fermentation standing of must from gamay grapes. American Journal of Enology and Viticulture 47(3):287-290.

Pezet, R., V. Pont \& K. Van-Hoan (1991). Evidence for oxidative detoxication of pterostilbene and resveratrol by a laccase-like stilbene oxidase produced by Botrytis cinerea Pers. Physiological and Molecular Plant Pathology 39:441-450. https://doi.org/10.1016/08855765(91)90010-F

Pezet, R., V. Pont \& P. Cuenat (1994). Method to determine resveratrol and pterostilbene in grape berries and wines using high-performance liquid chromatography and highly sensitive fluorimetric detection. Journal of Chromatography A 663:191-197.

https://doi.org/10.1016/0021-9673(94)85245-6

Pezet, R., K. Gindro, O. Viret \& J.-L. Spring (2004). Glycosylation and oxidative dimerization of resveratrol are respectively associated to sensitivity and resistance of grapevine cultivars to downy mildew. Physiological and Molecular Plant Pathology 65:297-303.

https://doi.org/10.1016/j.pmpp.2005.03.002

Pezzuto, J.M. (2011). The phenomenon of resveratrol: redefining the virtues of promiscuity. Annals of the New York Academy of Sciences 1215:123-130. https://doi.org/10.1111/j.1749-

6632.2010.05849.x

Phommalth, S., Jeong, Y.-S., Kim, Y-H., Dhakal, K.H \& Y-H. Hwang (2008). Effects of light treatment on isoflavone content of germinated soybean seeds. Journal of Agricultural and Food Chemistry 56(21):10123-10128. https://doi.org/10.1021/jf802118g

Pianjing, P., A. Thiantanawat, N. Rangkadilok, P. Watcharasit, C. Mahidol \& J. Satayavivad (2011). Estrogenic activities of sesame lignans and their metabolites on human breast cancer cells. Journal of Agricultural Food Chemistry 59(1):212-221. https://doi.org/10.1021/jf102006w

Piňeiro, Z., M. Palma \& C.G. Barroso (2006). Determination of trans-resveratrol in grapes by pressurised liquid extraction and fast high-performance liquid chromatography. Journal of Chromatography A 1110:61-65. https://doi.org/10.1016/j.chroma.2006.01.067

Pool, R.M., L.L. Creasy \& A.S. Frackelton (1981). Resveratrol and the viniferins, their application to screening for disease resistance in grape breeding programs. Vitis 20:136-145.

Potrebko, I. \& A.V.A. Resurreccion (2009). Effect of ultraviolet doses in combined ultravioletultrasound treatments on trans-resveratrol and trans-piceid contents in sliced peanut kernels. Journal of Agricultural and Food Chemistry 57:7750-7756. https://doi.org/10.1021/jf900667d

Potter, S.M., J.A. Baum, H.Y. Teng, R.J. Stillman, N.F. Shay \& J.W. Erdman (1998). Soy protein and isoflavones: their effects on blood lipids and bone density in postmenopausal women. American Journal of Clinical Nutrition 68:1375S-1379S.

Pour Nikfardjam, M.S. (2002). In: Polyphenole in Weissweinen und Traubensäften und ihre Veränderung im Verlauf der Herstellung. Germany: Tectum Verlag Marburg.

Pour Nikfardjam, M., K. Schmitt, E.H. Rühl, C.-D. Patz \& H. Dietrich (2000). Undersuchung rebsortenreiner Traubensäfte auf den Gehalt an Resveratrol-Derivaten. Deutshe LebensmittelRundschau 96:319-324.

Pour Nikfardjam, M.S., G. László \& H. Dietrich (2006a). Resveratrol-derivatives and antioxidative capacity in wines made from botrytized grapes. Food Chemistry 96:74-79. https://doi.org/10.1016/j.foodchem.2005.01.058

Pour Nikfardjam, M.S., L. Márk, P. Avar, M. Figler \& R. Ohmacht (2006b) Polyphenols, anthocyanins, and trans-resveratrol in red wines from the Hungarian Villány region. Food Chemistry 98:453-462. https://doi.org/10.1016/j.foodchem.2005.06.014

Poussier, M., M. Guilloux-Benatier, M. Torres, E. Heras \& M. Adrian (2003). Influence of different maceration techniques and microbial enzymatic activities on wine stilbene content. American Journal of Enology and Viticulture 54(4):261-266.

Pozo-Bayón, M.A., M.T. Hernández, P.J. Martin-Álvarez \& M.C. Polo (2003). Study of low molecular weight phenolic compounds during the aging of sparkling wines manufactured with red and white grape varieties. Journal of Agricultural and Food Chemistry 51:2089-2095. https://doi.org/10.1021/jfo21017z 
Primomo, V.S., V. Poysa, G.R. Ablett, C.-J. Jackson \& I. Rajcan (2005). Agronomic performance of recombinant inbred line populations segregating for isoflavone content in soyben seeds. Crop Science 45:2203-2211. https://doi.org/10.2135/cropsci2004.0610

Püssa, T., J. Floren, P. Kuldkepp \& A. Raal (2006). Survey of grapevine Vitis vinifera stem polyphenols by liquid chromatography - diode array detection-tandem mass spectrometry. Journal of Agricultural and Food Chemistry 54:7488-7494. https://doi.org/10.1021/jfo61155e

Püssa, T., P. Raudsepp, K. Kuzina \& A. Raal (2009). Polyphenolic composition of roots and petioles of Rheum rhaponticum L. Phytochemical Analysis 20(2):98-103.

https://doi.org/10.1002/pca.1102, http://www.ncbi.nlm.nih.gov/pubmed/18979462

Quinhone Júnior, A. \& E.I. Ida (2015). Profile of the contents of different forms of soybean isoflavones and the effect of germination time on these compounds and the physical parameters in soybean sprouts. Food Chemistry 166:173-178.

https://doi.org/10.1016/j.foodchem.2014.06.012

Ragab, A.S., J. van Fleet, B. Jankoski, J.-H- Park \& S.C. Bobzin (2006) Detection and quantitation of resveratrol in tomato fruit (Lycopersicon esculentum Mill.). Journal of Agricultural and Food Chemistry 54:7175-7179. https://doi.org/10.1021/jfo6og633

Reinli, K. \& G. Block (1996). Phytoestrogen content of foods - A compendium of literature values. Nutrition and Cancer 26:123-148. https://doi.org/10.1080/01635589609514470

Revilla, E. \& J.-M.Ryan (2000). Analysis of several phenolic compounds with potential antioxidant properties in grape extracts and wines by high-performance liquid chromatography-photodiode array detection without sample preparation. Journal of Chromatography A 881:461-469. https://doi.org/10.1016/S0021-9673(00)00269-7

Ribeiro, M.L.L., J.M.G. Mandarino, M.C. Carrăo-Panizzi, M.C.N. de Oliveira, C.B.H. Campo, A.L. Nepomuceno \& E.I. Ida (2007). Isoflavone content and $\beta$-glucosidase activity in soybean cultivars of different maturity groups. Journal of Food Composition and Analysis 20:19-24. https://doi.org/10.1016/j.jfca.2006.07.004

Ribeiro de Lima, M.T., P. Waffo-Téguo, P.L. Teissedre, A. Pujolas, J. Vercauteren, J.C. Cabanis \& J.M. Mérillon (1999). Determination of stilbenes (trans-astringin, cis- and trans-piceid, and cisand trans-resveratrol) in Portuguese wines. Journal of Agricultural and Food Chemistry 47:2666-2670. https://doi.org/10.1021/jf9900884

Ribeiro da Silva, R.L.M., E.A. Teixeira de Figueiredo, N.M. Silva Ricardo, I.G. Pinto Vieira, R. Wilane de Figueiredo, I.M. Brasil \& C.L. Gomes (2014). Quantification of bioactive compounds in pulps and by-products of tropical fruits from Brazil. Food Chemistry 143:398404. https://doi.org/10.1016/j.foodchem.2013.08.001, http://www.ncbi.nlm.nih.gov/pubmed/24054258

Riedl K.M., J.H. Lee, M. Renita, S.K. St Martin, S.J. Schwartz \& Y. Vodovotz (2007). Isoflavone profiles, phenol content, and antioxidant activity of soybean seeds as influenced by cultivar and growing location in Ohio. Journal of the Science of Food and Agriculture 87(7):1197-1206. https://doi.org/10.1002/jsfa. 2795

Rimando, A.M. \& D.L. Barney (2005). Resveratrol and naturally occurring analogues in Vaccinium species. Proceedings of the WOCMAP III, Vol. 6 (Traditional Medicine \& Neutraceuticals), U.R. Palaniswamy, L.E. Craker and Z.E. Gardner, Acta Horticulturae (International Society for Horticultural Science, Belgium) 680:137-143.

https://doi.org/10.1766o/actahortic.2005.680.20

Rimando, A.M., W. Kalt, J.B. Magee, J. Dewey \& J.R. Ballington (2004). Resveratrol, pterostilbene, and piceatannol in vaccinium berries. Journal of Agricultural and Food Chemistry 52(15):4713-4719. https://doi.org/10.1021/jfo40095e,

http://www.ncbi.nlm.nih.gov/pubmed/15264904

Rodríguez-Delgado, S. Malovaná, M.A., J.P. Pérez, T. Borges \& F.J. Garcia Montelongo (2001). Separation of phenolic compounds by high-performance liquid chromatography with absorbance and fluorimetric detection. Journal of Chromatography A 912:249-257. https://doi.org/10.1016/S0021-9673(01)00598-2 
Rodríguez-Delgado, M.A., G. Gonzalez, J.P. Pérez-Truhillo \& F.J. Garcia-Montelongo (2002). Trans-resveratrol in wines from the Canary Islands (Spain): Analysis by high performance liquid chromatography. Food Chemistry 76:371-375. https://doi.org/10.1016/So308-

8146(01)00258-8

Roggero, J.-P. (1996). Évolution des teneurs en resvératrol et en picéide dans des vins en cours de fermentation ou de vieillisement. Comparaison des cépages genache et mourvèdre.

Sciences des Aliments 16:631-642.

Roggero, J.-P. \& P. Archier (1994). Sosage du resvératrol et de l'un de ses glycosides dans les vins. Sciences des Aliments 14:99-107.

Roggero, J.-P. \& C. Garcia-Parrilla (1995). effects of ultravioltet irradiation on resveratrol and changes in resveratrol an various of its derivatives in th skins of ripening grapes. Sciences des Aliments 15:411-422.

Roldán, A., V. Palacios, I. Caro \& L. Pérez (2003). Resveratrol content of Palomino fino grapes: influence of vintage and fungal infection. Journal of Agricultural and Food Chemistry 51(5):1464-1468. https://doi.org/10.1021/jfo20774U, http://www.ncbi.nlm.nih.gov/pubmed/12590499

Romani, A., P. Vignolini, C. Galardi, C. Arnoldi, C. Vazzana \& D. Heimler (2003). Polyphenolic content in dofferent plant parts of soy cultivars grown under natural conditions. Journal of Agricultural and Food Chemistry 51:5301-5306. https://doi.org/10.1021/jfo212136

Romero-Pérez, A.I., R.M. Lamuela-Raventós, S. Buxaderas \& M.C. de La Torre-Boronat (1996a) Resveratrol and piceid as varietal markers of white wines. Journal of Agricultural and Food Chemistry 44(8):1975-1978. https://doi.org/10.1021/jf960211g

Romero-Pérez, A.I., R.M. Lamuela-Raventós, A. Waterhouse \& M.C. de La Torre-Boronat (1996b) Levels of cis- and trans-resveratrol and their glucosides in white and rose Vitis vinifera wines from Spain. Journal of Agricultural and Food Chemistry 44:2124-2128.

Romero-Pérez, A.I., M. Ibern-Gómez, R.M. Lamuela-Raventós \& M.C. de La Torre-Boronat (1999) Piceid, the major resveratrol derivative in grape juices. Journal of Agricultural and Food Chemistry 47(4):1533-1536. https://doi.org/10.1021/jf9810249,

http://www.ncbi.nlm.nih.gov/pubmed/10564012

Romero-Pérez, A.I., R.M. Lamuela-Raventós, C. Andrés-Lacueva C \& M.C. de La Torre-Boronat (2001). Method for the quantitative extraction of resveratrol and piceid isomers in grape berry skins. Effect of powdery mildew on the stilbene content. Journal of Agricultural and Food Chemistry 49(1):210-215. https://doi.org/10.1021/jfooo7450, http://www.ncbi.nlm.nih.gov/pubmed/11170579

Ros-Barcelo, A., F. Pomar, M. Lopez-Serrano \& M.A. Pedreno (2003). Peroxidase_a multifunctional enzyme in grapevines. Functional Plant Biology 30:577-591. https://doi.org/10.1071/FP02096

Routledge, E., R. White, M. Parker \& J. Sumpter (2000). Differential effects of xenoestrogens on coactivator recruitment by estrogen receptor (ER) $\alpha$ and ER $\beta$. The Journal of Biological Chemistry 275(46):35986-35993. https://doi.org/10.1074/jbc.Moo6777200

Rowland I.R., H. Wiseman, T.A.B. Sanders, H. Adlercreutz \& E.A. Bowey (2000). Interindividual variation in metabolism of soy isoflavones and lignans: influence of habitual diet on equol production by the gut microflora. Nutrition and Cancer 36(1):27-32.

https://doi.org/10.1207/S15327914NC3601_5

Rudolf, J.R. \& A.V.A. Resurreccion (2005). Elicitation of resveratrol in peanut kernels by application of abiotic stresses. Journal of Agricultural and Food Chemistry 53:10186-10192. https://doi.org/10.1021/jfo506737

Rupprich, N., H. Hildebrand \& H. Kindl (1980) Substrate specificity in vivo and in vitro in the formation of stilbenes. Biosynthesis of rhaponticin. Archives of Biochemistry and Biophysics 200:72-78. https://doi.org/10.1016/0003-9861(80)90332-X 
Saarinen, N., C. Bingham, S. Lorenzetti, A. Mortensen, S. Mäkelä, P. Penttinen, I. Sørensen, L. Valsta, F. Virgili, G. Vollmer, A. Wärri \& O. Zierau (2006). Tools to evaluate estrogenic potency of dietary phytoestrogens: a consensus paper from the EU thematic network "phytohealth". Genes \& Nutrition 1(3-4):143-158. https://doi.org/10.1007/BFo2829964

Sakkiadi, A.-V., M.N. Stavrakakis \& S.A. Haroutounian (2001). Direct HPLC assay of five biologically interesting phenolic antioxidants in varietal Greek red wines. LebensmittelWissenschaft und Technologie 34:410-413. https://doi.org/10.1006/fstl.2001.0792

Sakakibara, H., Y. Honda, S. Nakagawa, H. Ashida \& K. Kanazawa (2003). Simultaneous determination of all polyphenols in vegetables, fruits, and teas. Journal of Agricultural and Food Chemistry51:571-581. https://doi.org/10.1021/jfo20926I

Sakthivelu, G., M.K. Akitha Devi, P. Giridhar, T. Rajasekaran, G.A. Ravishankar, M.T. Nikolova, G.B. Angelov, R.M. Todorova \& G.P. Kosturkova (2008). Isoflavone composition, phenol content, and antioxidant activity of soybean seeds from India and Bulgaria. Journal of Agricultural and Food Chemistry 56:2090-2095. https://doi.org/10.1021/jfo72939a

Saloniemi, H., K. Wähälä, P. Nykänen-Kurki, K. Kallela \& I. Saastamoinen (1995). Phytoestrogen content and estrogenic effect of legume fodder. Proceedings of the Society of Experimental Biology and Medicine 208(1):13-17. https://doi.org/10.3181/00379727-208-43825

Sanderman, H. \& W. Wellerman (1988). Risikobewertung kunstlicher herbizid-resistenz. In: Biologische Sicherheit, Bundesministerium für Forschung und Technologie, Bonn, Germany, pp. 285-292.

Sanders, T.H., R.W. McMichael Jr \& K.W. Hendrix (2000). Occurrence of resveratrol in edible peanuts. Journal of Agricultural and Food Chemistry 48:1243-1246.

https://doi.org/10.1021/jf990737b

Santell, R.C., Y.C. Chang, M.C. Nair \& W.G. Helferich (1977). Dietary genistein exerts estrogenic effects upon the uterus, mammary gland and the hypothalamic/pituitary axis in rats. Journal of Nutrition 127(2):263-269.

Sarig, P., T. Zahavi, Y. Zutkhi, S. Yannai, N. Lisker \& R. Ben-Arie (1996). Ozone for control of post-harvest decay of table grapes caused by Rhizopus stolonifer. Physiological and Molecular Pathology 48:403-415. https://doi.org/10.1006/pmpp.1996.0032

Sarig, P., Y. Zutkhi, A. Monjauze, N. Lisker \& R. Ben-Arie (1997). Phytoalexin elicitation in grape berries and their susceptibility to Rhizopus stolonifer. Physiological and Molecular Plant Pathology 50:337-347. https://doi.org/10.1006/pmpp.1997.0089

Sato, M., Y. Suzuki, T. Okuda \& K. Yokotsuka (1997). Contents of resveratrol, piceid, and their isomers in commercially available wines made from grapes cultivated in Japan. Bioscience, Biotechnology Biochemistry 61(11):1800-1805. https://doi.org/10.1271/bbb.61.1800, http://www.ncbi.nlm.nih.gov/pubmed/9404057

Sautter, C.K., S. Denardin, A.O: Alves, C.A. Mallmann, N.G. Penna \& L.H. Hecktheuer (2005). Determinaçaão de resveratrol em sucos de uva no Brasil. Food Science and Technology (Campinas) 25(3):437-442. https://doi.org/10.1590/S0101-20612005000300008

Sbaghi, M., P. Jeandet, B. Faivre, R. Bessis \& J.C. Fourniooux (1995). Development of methods using phytoalexin (resveratrol) assessment as a selection criterion to screen grapevine in vitro cultures for resistance to grey mould (Botrytis cinerea). Euphytica 86:41-47.

https://doi.org/10.1007/BFoo035937

Sbaghi, M., P. Jeandet, R. Bessis \& P. Leroux (1996). Degradation of stilbene-type phytoalexins in relation to the pathogenicity of Botrytis cinerea to grapevine. Plant Pathology 45:139-144. https://doi.org/10.1046/j.1365-3059.1996.do1-101.x

Schmidlin, L., A. Poutaraud, P. Claudel, P. Mestre, E. Prado, M. Santos-Rosa, S. WiedemannMerdinogly, F. Karst, D. Merdinoglu \& P. Hugueney (2008). A stress-inducible resveratrol $O$ methyltransferase involved in the biosynthesis of pterostilbene in grapevine. Molecular Plant Microbe Interactions 19:429-440. https://doi.org/10.1104/pp.108.126003

Schnee, S., O. Viret \& K. Gindro (2008). Role of stilbenes in the resistance of grapevinte to powdery mildew. Physiological and Molecular Plant Pathology 72:128-133.

https://doi.org/10.1016/j.pmpp.2008.07.002 
Schwartz H. \& G. Sontag (2006). Determination of secoisolariciresinol, lariciresinol and isolariciresinol in plant foods by high performance liquid chromatography coupled with coulometric electrode array detection. Journal of Chromatography 838(2):78-85. https://doi.org/10.1016/j.jchromb.2006.03.058

Schwartz, H., G. Sontag \& J. Plumb (2009). Inventory of phytoestrogen databases. Food Chemistry 113(3):736-747. https://doi.org/10.1016/j.foodchem.2008.09.051

Schwekendiek, A., O. Spring, A. Heyerick, B. Pickel, N.T. Pitsch, F. Peschke, D. de Keukeleire \& G. Weber (2007) Constitutive expression of a grapevine stilbene synthase gene in transgenic hop (Humulus lupulus L.) yield resveratrol and its derivatives in substantial quantities. Journal of Agricultural and Food Chemistry 55:7002-7009. https://doi.org/10.1021/jfo70509e

Scott, W.O. \& S.R. Aldridge (1983) Modern Soybean Production. S\&A Publications, Champaign, Illinois.

Seguin, P. W. Zheng, D.L. Smith \& W. Deng (2004). Isoflavone content of soybean cultivars grown in eastern Canada. Journal of the Science of Food and Agriculture 84:1327-1332. https://doi.org/10.1002/jsfa.1825

Selma, M.V., P.M. Freitas, L. Almela, R. González-Barrio, J.C. Espín, TSuslow, F. TomásBarberán \& M.I. Gil (2008). Ultraviolet-C and induced stilbenes control ochratoxigenic Aspergillus in grapes. Journal of Agricultural and Food Chemistry 56(21):9990-9996. https://doi.org/10.1021/jf8018062, http://www.ncbi.nlm.nih.gov/pubmed/18841974

Sergiel, I., P. Pohl \& M. Biesaga (2014). Characterisation of honey according to their content of pehnolic compounds using high performance liquid chromatography/tandem mass spectrometry. Food Chemistry 145:404-408. https://doi.org/10.1016/j.foodchem.2013.08.068

Setchell, K.D.R., S.P. Borriello, P. Humle, D.N. Kirk \& M. Axelson (1984). Nonsteroidal estrogens of dietary origin: possible roles in hormone-dependent disease. American Journal of Clinical Nutrition 40(3):569-578.

Setchell, K.D.R. \& S. J. Cole (2003). Variations in isoflavone levels in soy foods and soy protein isolates and issues related to isoflavone databases and food labelling. Journal of Agricultural and Food Chemistry 51:4146-4155. https://doi.org/10.1021/jfo26199b

Setchell K.D.R., C. Childress, L. Zimmer-Nechemias \& J. Cai (1999). Method for measurement of dietary secoisolariciresinol using HPLC with multichannel electrochemical detection. Journal of Medicinal Food 2(3-4):193-198. https://doi.org/10.1089/jmf.1999.2.193

Setchell K.D.R., N.M. Brown, L. Zimmer-Nechemias, W.T. Brashear, B.E. Wolfe, A.S. Kirschner $\&$ J.E. Heubi (2002). Evidence for lack of absorption of soy isoflavone glycosides in humans, supporting the crucial role of intestinal metabolism for bioavailability. The American Journal of Clinical Nutrition 76(2):447-453.

Shao, Y., P. Marriott \& H. Hügel (2003). Solid-phase microextraction - on-fibre derivatization with comprehensive two dimensional gas chromatography analysis of trans-resveratrol in wine. Chromatographia 57(Suppl):S349-S353. https://doi.org/10.1007/BFo2492127

Shih, C.-H., Chen, Y., Wang, M., Chu, I.K. \& C. Lo (2008). Accumulation of isoflavone genistin in transgenic tomato plants overexpressing a soybean isoflavone synthase gene. Journal of Agricultural and Food Chemistry 56:5655-5661. https://doi.org/10.1021/jf800423U

Siemann, E.H. \& L.L. Creasy (1992). Concentration of the phytoalexin resveratrol in wine. American Journal of Enology and Viticulture 43:49-52.

Silva, C.G., J. Monteiro, R.R. Marques, A.M. Silva, C. Martínez, M. Canle \& J.L. Faria (2013). Photochemical and photocatalytic degradation of trans-resveratrol. Photochemical \& Photobiological Sciences 12(4):638-644. https://doi.org/10.1039/C2PP25239B, http://www.ncbi.nlm.nih.gov/pubmed/23064356

Simonne, A.H., Smith, M., Weaver, D.B., Vail, T., Barnes, S. \& Wei, C.I. (2000). Retention and changes of soy isoflavones and carotenoids in immature soybean seeds (edamame) during processing. Journal of Agricultural and Food Chemistry 48:6061-6069.

https://doi.org/10.1021/jfooo247f 
Simons, R., J.-P. Vinchen, N. Roidos, T.F.H. Bovee, M. de lersel, M.A. Verbruggen \& H. Gruppen (2011). Increasing soy isoflavonoid content and diversity by simultaneous malting and challenging by a fungus to modulate estrogenicity. Journal of Agricultural and Food Chemistry 59:6748-6758. https://doi.org/10.1021/jf2010707

Slavin J.L., S.C. Karr, A.M. Hutchins \& J.W. Lampe (1998). Influence of soybean processing, habitual diet, and soy dose on urinary isoflavonoid excretion. The American Journal of Clinical Nutrition 68(6):1492S-1495S.

Smeds, A.I., P.C. Eklund, R.E. Sjöholm, S.M. Willför, S. Nishibe, T. Deyama \& B.R. Holmbom (2007). Quantification of broad spectrum of lignans in cereals, oilseeds, and nuts. Journal of Agricultural and Food Chemistry 55(4):1337-1346. https://doi.org/10.1021/jfo629134

Smeds A.I., L. Jauhiainen, E. Tiumola \& P. Peltonen-Sainio (2009). Characterization of variation in the lignan content and composition of winter rye, spring wheat, and spring oat. Journal of Agricultural and Food Chemistry 57(13):5837-5842. https://doi.org/10.1021/jf9004274

Smeds A.I. P.C. Eklund \& S.M. Willför (2012). Content, composition, and stereochemical characterisation of lignans in berries and seeds. Food Chemistry 134(4):1991-1998. https://doi.org/10.1016/j.foodchem.2012.03.133

Snyder, H.E. \& L.A. Wilson (2003). Processing for the food industry. Encyclopedia of Food Science and Nutrition, p. 5383-5389. https://doi.org/10.1016/Bo-12-227055-X/01110-X

Sobolev, .V.S \& R.J. Cole (1999). trans-resveratrol content in commercial peanuts and peanut products. Journal of Agricultural and Food Chemistry 47(4):1435-1439.

https://doi.org/10.1021/jf9809885, http://www.ncbi.nlm.nih.gov/pubmed/10563995

Sobolev, V.S., R.J. Cole \& J.W. Dorner (1995). Isolation, purification, and liquid chromatographic determination of stilbene phytoalexins in peanuts. Journal of AOAC International 78(5):11771182.

Sochor, J., O. Zitka, H. Skutkova, D. Pavlik, P. Babula, B. Krska, A. Horna, V. Adam, I. Provaznik $\&$ R. Kizek (2010). Content of phenolic compounds and antioxidant capacity in fruits of apricot genotypes. Molecules 15(9):6285-6305. https://doi.org/10.3390/molecules15096285, http://www.ncbi.nlm.nih.gov/pubmed/20877223

Soleas, G.J., D.M. Goldberg, E.P. Diamandis, A. Karumanchiri, J. Yan \& E. Ng (1995a). A derivatized gas chromatographic-mass spectrometric method for the analysis of both isomers of resveratrol in juice and wine. American Journal of Enology and Vitic 46(3):346-352.

Soleas, G.J., D.M. Goldberg, A. Karumanchiri, E.P. Diamandis \& E. Ng (1995b). Influences of viticultural and oenological factors on changes in cis- and trans-resveratrol in commercial wines. Journal of Wine Research 6:107-121. https://doi.org/10.1080/09571269508718028

Soleas, G.J., D.M. Goldberg, E. Ng, A. Karumanchiri, E. Tsang \& E.P. Diamandis (1997). Comparative evaluation of four methods for assay of cis- and trans-resveratrol. American Journal of Enology and Vitic 48(2):169-176.

Song, T., Barua, K., Buseman, G. \& P.A. Murphy (1998). Soy isoflavone analysis: quality control and a new internal standard. American Journal of Clinical Nutrition 68 (Suppl):1474S-1479S.

Song, T.T., Hendrich, S. \& P.A. Murphy (1999). Estrogenic activity of glycitein, a soy isoflavone. Journal of Agricultural and Food Chemistry 47:1607-1610. https://doi.org/10.1021/jf981054j

Song, W., H.J. Wang, P. Bucheli, P.F. Zhang, D.Z. Wei Y.H. Lu (2009). Phytochemical profiles of different mulberry (Morus sp.) species from China. Journal of Agricultural and Food Chemistry 57(19):9133-9140. https://doi.org/10.1021/jf981054j, http://www.ncbi.nlm.nih.gov/pubmed/19761189

Soto, A., C. Sonnenschein, K. Chung, M. Fernandez, N. Olea \& F. Olea Serrano (1995). The Escreen assay as a tool to identify estrogens: an update on estrogenic environmental pollutants. Environmental Health Perspectives 103:113-122.

https://doi.org/10.1289/ehp.9510357113

Souto, A.A., M.C. Carneiro, M. Seferin, M.J.H. Senna, A. Conz \& K. Gobbi (2001). Determination of trans-resveratrol concentrations in Brazilian red wines by HPLC. Journal of Food Consumption and Analysis 14:441-445. https://doi.org/10.1006/jfca. 2000.0970 
Stein, U. \& G. Hoos (1984). Induktions und Nachweismethoden für Stilbene bei Vitaceen. Vitis 23:179-194.

Steinshamn, H., S. Purup, E. Thuen \& J. Hansen.Møller (2008). Effects of clover-grass silages and concentrate supplementation on the content of phytoestrogens in dairy cow milk. Journal of Dairy Science 91(7):2715-2725. https://doi.org/10.3168/jds.2007-0857

Stervbo, U., O. Vang \& C. Bonnesen (2007). A review of the content of the putative chemopreventive phytoalexin resveratrol in red wine. Food Chemistry 101:449-457. https://doi.org/10.3168/jds.2007-0857

Stintzing, F.C., Hoffmann, M. \& R. Carle (2006). Thermal degradation kinetis of isoflavone aglycones from soy and red clover. Molecular Nutrition \& Food Research 50:373-377. https://doi.org/10.1002/mnfr.200500187

Subramanian, S., Hu, X., Lu, G., Odell, J.T. \& O. Yu (2004). The promoters of two isoflavone synthase genes respond differentially to nodulation and defense signals in transgenic soybean roots. Plant Molecular Biology 54:623-639.

https://doi.org/10.1023/B:PLAN.0000040814.28507.35

Sun, B., C. Ferrao \& M.I. Spranger (2003). effect of wine style and winemaking technology on resveratrol levels in wines. Ciencia e Técnica Vitivinicola 18(2):77-91.

Takaoka, M. (1940). The phenolic substances of white hellebore (Veratrum Grandiflorum Loes. Fill). V. Nippon Kagaku Kaishi 61:1067-1069. https://doi.org/10.1246/nikkashi1921.61.1067

Taylor, N.B., Fuchs, R.L., MacDonald, J., Shariff, A.R. \& S.R. Padgette (1999). Compositional analysis of glyphosate-tolerant soybeans treated with glyphosate. Journal of Agricultural and Food Chemistry 47:4469-4473. https://doi.org/10.1021/jf990056g

Tedesco, I., A. Nappo, F. Petitto, G. lacomino, F. Nazzaro, R. Palumbo \& G.L., Russo (2005). Antioxidant and cytotoxic properties of lyophilized beer extracts on HL-6o cell line. Nutrition and Cancer 52(1):74-83. https://doi.org/10.1207/s15327914nc5201_10

Tepavcevic, V., Atanackovic, M., Miladinovic, J., Malencic, D., Popovic, J. \& J. Cvejic (2010). isoflavone composition, total polyphenolic content, and antioxidant activity in soybeans of different origin. Journal of Medicinal Food 13(3):657-664. https://doi.org/10.1089/jmf.2009.0050

Tetens I., A. Turrini, H. Tapanainen, T. Christensen, J.W. Lampe, S. Fagt, N. Håkansson, A. Lundquist, J. Hallund, L.M. Valsta \& The Phytohealth WPI working group (2013). Dietary intake and main sources of plant lignans in five European countries. Food and Nutrition Research 57:19805 - DOI: 10.3402/fnr.v57io.19805. https://doi.org/10.3402/fnr.v57io.19805

Thompson, L.U., P. Robb, M. Serraino \& F. Cheung (1991). Mammalian lignan production from various foods. Nutrition and Cancer 16(1):43-52. https://doi.org/10.1080/01635589109514139

Thompson L.U., S.E. Rickard, F. Cheung, E.O. Kenaschuk \& W.R. Obermeyer (1997). Variability in anticancer lignan levels in flaxseed. Nutrition and Cancer 27(1):26-30. https://doi.org/10.1080/01635589709514497

Thompson, L.U., B.A. Boucher, Z. Liu, M. Cotterchio \& N. Kreiger (2006). Phytoestrogen content of foods consumed in Canada, including isoflavones, lignans and coumestan. Nutrition and Cancer 54(2):184-201. https://doi.org/10.1207/s15327914nc5402_5

Threlfall, R.T., J.R. Morris \& A. Mauromoustakos (1999). Effect of variety, ultraviolet light exposure, and enological methods on the trans-resveratrol level of wine. American Journal of Enology and Viticulture 50(1):57-64.

Tinwell H., A.R. Soames, J.R. Foster \& J. Ashby (2000). Estradiol-type activity of coumestrol in mature and immature ovariectomized rat uterotrophic assays. Environmental Health Perspectives 108(7):631-634. https://doi.org/10.1289/ehp.00108631

Tokuşoğlu, Ö., M.K. Ünal \& F. Yemiş (2005) Determination of the phytoalexin resveratrol $\left(3,5,4^{\prime}-\right.$ trihydroxystilbene) in peanuts and pistachios by high-performance liquid chromatographic diode array (HPLC-DAD) and gas chromatography-mass spectrometry (GC-MS). Journal of Agricultural and Food Chemistry 53:5003-5009. https://doi.org/10.1021/jf050496 
Tominaga, S., T. Sugahara, S. Nishimoto, M. Yamawaki, Y. Nakashima, T. Kishida, K. Akiyama, M. Maruyama \& S. Yamauchi (2009). The effect of secoisolariciresinol on 3T3-L1 adipocytes and the relationship between molecular structure and activity. Bioscience, Biotechnology, and Biochemistry 73(1):35-39. https://doi.org/10.1271/bbb.80393

Trela, B.C. \& A.L. Waterhouse (1996). Resveratrol: Isomeric molar absorptivities and stability. Journal of Agricultural and Food Chemistry 44:1253-1257. https://doi.org/10.1021/jf9504576

Tsukamoto, C., Shimada, S., Igita, K., Kudou, S., Kokubun, M., Okubo, K. \& K. Kitamura (1995). Factors affecting isoflavone content in soybean seeds: changes in isoflavones, saponins, and composition of fatty acids ar different temperatures during seed development. Journal of Agricultural and Food Chemistry 43(5):1184-1192. https://doi.org/10.1021/jfoo053a012

Ungar, Y., Osundahunsi, O.F: \& E. Shimoni (2003). Thermal stability of genistein and daidzein and its effect on their antioxidant activity. Journal of Agricultural and Food Chemistry 51:43944399. https://doi.org/10.1021/jf034021Z

Vacca, V., L. Leccis, P. Fenu, L. Pretti \& G.A. Farris (1997) Wine yeast and resveratrol content. Biotechnology Letters 19(6):497-498. https://doi.org/10.1023/A:1018316815670

Vaher, M. \& M. Koel (2003). Separation of polyphenolic compounds extracted from plant matrices using capillary electrophoresis. Journal of Chromatography A 990(1-2):225-230. https://doi.org/10.1016/S0021-9673(02)02013-7, http://www.ncbi.nlm.nih.gov/pubmed/12685601

Vastano, B.C., Y. Chen, N. Zhu, C.-T. Ho, Z. Zhou \& R.T. Rosen (2000). Isolation and identification of stilbenes in two varieties of Polygonum cuspidatum. Journal of Agricultural and Food Chemistry 48:253-256. https://doi.org/10.1021/jf9909196

Vezzulli, S., S. Civardi, F. Ferrari \& L. Bavaresco (2007a). Methyl jasmonate treatment as a trigger of resveratrol synthesis in cultivated grapevine. American Journal of Enology and Viticulture 58(4):530-533.

Vezzulli, S., P. Battilani \& L. Bavaresco (2007b). Stilbene-synthase gene expression after Aspergillus carbonarius infection in grapes. American Journal of Enology and Viticulture 58(1):132-134.

Vidhyasekaran, P., D. Lalithakumari \& C.V. Govindaswamy (1972). Production of a phytoalexin in groundnut due to storage fungi. Indian Phytopathology 25:240-245.

Viñas, P., C. López-Erroz, J.J. Marin-Hernández \& M. Hernández-Córdoba (2000). Determination of phenols in wines by liquid chromatography with photodiode array and fluorescence detection. Journal of Chromatography A 871:85-93.

https://doi.org/10.1016/So021-9673(99)01087-0

Viñas, P., N. Campillo, M. Hernández-Pérez \& M. Hernández-Córdoba (2008). A comparison of solid-phase microextraction and stir bar sorptive extraction coupled to liquid chromatography for the rapid analysis of resveratrol isomers in wines, musts and fruit juices. Analytica Chimica Acta 611(1):119-125. https://doi.org/10.1016/j.aca.2008.01.072, http://www.ncbi.nlm.nih.gov/pubmed/18298976

Vitrac, X., J.P. Monti, J. Vercauteren, G. Deffieux \& J.-M. Mérillon (2002). Direct liquid chromatographic analysis of resveratrol derivatives and flavanonols in wines with absorbance and fluorescence detection. Analytica Chimica Acta 458:103-110.

https://doi.org/10.1016/S0003-2670(01)01498-2

Vlase, L., B. Kiss, S.E. Leucuta \& S. Gocan (2009). A rapid method for determination of resveratrol in wines by HPLC-MS. Journal of Liquid Chromatography \& Related Technologies. 32:2105-2121. https://doi.org/10.1080/10826070903126989

Vrhovsek, U., R. Eder \& S. Wendelin (1995). The occurrence of trans-resveratrol in Slovenian red and white wines. Acta Alimentaria 24(2):203-212.

Vrhovsek, U., S. Wendelin \& R. Eder (1997). Effects of various vinification techniques on the concentration of cis- and trans-resveratrol and resveratrol glucoside isomers in wine. American Journal of Enology and Viticulture 48(2):214-219. 
Vyn, T.j., Yin, X., Bruulsema, T.W., Jackson, C.-J., Rajcan, I. \& S.M. Brouder (2002). Potassium fertilization effects on isoflavone concentrations in soybean [Glycine max (L.) Merr.]. Journal of Agricultural and Food Chemistry 50:3501-3506. https://doi.org/10.1021/jfo200671

Wang, H.-J. \& P.A. Murphy (1994a). Isoflavone composition of American and Japanese soybeans in lowa: effects of variety, crop year, and location. Journal of Agricultural and Food Chemistry 42(8):1674-1677. https://doi.org/10.1021/jfooo44a017

Wang, H.-J. \& P.A. Murphy (1994b). Isoflavone content in commercial soybean food. Journal of Agricultural and Food Chemistry 42:1666-1673. https://doi.org/10.1021/jfooo44a016

Wang, J. \& P. Sporns (2000). MALDI-TOF MS analysis of isoflavones in soy products. Journal of Agricultural and Food Chemistry 48:5887-5992. https://doi.org/10.1021/jfooo8947

Wang, G., Kuan, S.S., Francis, O.J., Ware, G.M. \& A.S. Carman (1990). A simplified HPLC method for the determination of phytoestrogens in soybean and its processed products. Journal of Agricultural and Food Chemistry 38(1):185-190. https://doi.org/10.1021/jfooog1a041

Wang, C., M. Sherrard, S. Pagadala, R. Wixon \& R.A. Scott (2000). Isoflavone content among maturity group o to II soybeans. Journal of American Oil Chemist's Society 77(5):483-487. https://doi.org/10.1007/s11746-000-0077-6

Wang, C-C., J.K. Prasain \& S. Barnes (2002a). Review of the methods used in the determination of phytoestrogens. Journal of Cromatography 777(1-2):3-28. https://doi.org/10.1016/s15700232(02)00341-0

Wang, Y., F. Carana, Y. Yang, R. Roderick, R.B. van Breemen (2002b). An LC-MS method for analysing total resveratrol in grape juice, cranberry juice, and wine. Journal of Agricultural and Food Chemistry 50:431-435. https://doi.org/10.1021/jfo10812U

Wang, S.Y., C.T. Chen, C.Y. Wang \& P. Chen (2007). Resveratrol content in strawberry fruit is affected by preharvest conditions. Journal of Agricultural and Food Chemistry 55(20):82698274. https://doi.org/10.1021/jfo71749x, http://www.ncbi.nlm.nih.gov/pubmed/17803264

Wang, L., M. Xu, C. Liu, J. Wang, H. Xi, B. Wu, W. Loescher, W. Duan, P. Fan \& S. Li (2013a). Resveratrols in grape berry skins and leaves in vitis germplasm. PLoS One 8(4):e61642. https://doi.org/10.1371/journal.pone.0061642, http://www.ncbi.nlm.nih.gov/pubmed/23637874

Wang, M.L., C.Y. Chen, B. Tonnis, N.A. Barkley, D.L. Pinnow, R.N. Pittman, J. Davis, C.C. Holbrook, H.T. Stalker \& G.A. Pederson (2013b). Oil, fatty acid, flavonoid, and resveratrol content variability and FAD2A functional SNP genotypes in the U.S. peanut mini-core collection. Journal of Agricultural and Food Chemistry 61(11):2875-2882. https://doi.org/10.1021/jf305208e, http://www.ncbi.nlm.nih.gov/pubmed/23379758

Wasternack, C. (2007). Jasmonates: An updatae on biosynthesis, signal transduction and action in plant stress response, growth and development. Annals of Botany 100:681-697. https://doi.org/10.1093/aob/mcmo79

Westphal, E. (1974). Pulses in Ethiopia, their taxonomy and agricultural significance. Agri. Res. Rep. (Wageningen, The Netherlands) 815:159-176.

Whent M., J. Lv, D.L. Luthria, W. Kenworthy \& L. Yu (2011). Isoflavone composition and antioxidant capacity of modified-lipoxygenase soybeans grown in Maryland. Journal of Agricultural and Food Chemistry 59(24):12902-12909. https://doi.org/10.1021/jf2038235

Whitten, P.L. \& F. Naftolin (1998). Reproductive actions of phytoestrogens. Baillieres Clinical Endocrinology and Metabolism 12(4):667-69o. https://doi.org/10.1016/So950-351X(98)80010-4

Wightman, J.D., S.F. Price, B.T. Watson \& R.E. Wrolstad (1997). Some effects of processing enzymes on anthocyanins and phenolics in Pinot noir and Cabernet Sauvignon Wines. American Journal of Enology and Viticulture 48(1):39-48.

Wiese, W., B. Vornam, E. Krause \& H. Kindl (1994). Structural organization and differential expression of three stilbene synthase genes located on a $13 \mathrm{~kb}$ grapevine DNA fragment. Plant Molecular Biology 26:667-677. https://doi.org/10.1007/BFoo013752

Woraratphoka, J., K.-O. Intarapichet \& K. Indrapichate (2007). Phenolic compounds and antioxidative properties of selected wines from the northeast of Thailand. Food Chemistry 104:1485-1490. https://doi.org/10.1016/j.foodchem.2007.02.020 
Wu, Z., L. Song, S. Feng, Y. Liu, G. He, Y. Yioe, S.Q. Liu \& D. Huang (2012). Germination dramatically increases isoflavonoid content and diversity in chickpea (Cicer arietinum L.) seeds. Journal of Agricultural and Food Chemistry 60:8606-8615.

https://doi.org/10.1021/jf3021514

Xie, L. \& B.W. Bolling (2014). Characterisation of stilbenes in California almonds (Prunus dulcis) by UHPLC-MS. Food Chemistry 148:300-306. https://doi.org/10.1016/j.foodchem.2013.10.057, http://www.ncbi.nlm.nih.gov/pubmed/24262561

Xu, Z., Wu, Q. \& J.S. Godber (2002). Stabilities of daidzin, genistin, and generation of derivatives during heating. Journal of Agricultural and Food Chemistry 50:7402-7406. https://doi.org/10.1021/jfo25626i

Xu, B. \& S.K. Chang (2008a). Total phenolics, phenolic acids, isoflavones, and anthocyanins and antioxidant properties of yellow and black soybeans as affected by thermal processing. Journal of Agricultural and Food Chemistry 56:7165-7175. https://doi.org/10.1021/jf8012234

Xu, B. \& S.K. Chang (2008b). Antioxidant capacity of seed coat, dehulled bean, and whole black soybeans in relation to their distributions of total phenolics, phenolic acids, anthocyanins and isoflavones. Journal of Agricultural and Food Chemistry 56:8365-8373. https://doi.org/10.1021/jf801196d

Xu, B. \& S.K. Chang (2008c). Characterization of phenolic substances and antioxidant properties of food soybeans grown in the North Dakota - Minnesota region. Journal of Agricultural and Food Chemistry 56:9102-9113. https://doi.org/10.1021/jf801451k

Yang, B., A-M. Linko, H. Adlercreutz \& H. Kallio (2006). Secoisolariciresinol and matairesinol of sea buckthorn (Hippophaë rhamnoides L.) berries of different subspecies and harvesting times. Journal of Agricultural and Food Chemistry 54(21):8065-8070. https://doi.org/10.1021/jfo61904s

Yoo, Y.J., P.D. Prenzler, A.J. Saliba \& D. Ryan (2011). Assessment of some Australian red wines for price, phenolic content, antioxidant activity, and vintage in relation to functional food prospects. Journal Food Science 76(9):C1355-C1364. https://doi.org/10.1111/j.17503841.2011.02429.x, http://www.ncbi.nlm.nih.gov/pubmed/22416699

Yu, O., Jung, W., Shi, J., Croes, R.A., Fader, G.M., McGonigle, B. \& J.T. Odell (2000). Production of the isoflavones genistein and daidzein in non-legume dicot and monocot tissues. Plant Physiology124:781-793. https://doi.org/10.1104/pp.124.2.781

Zhang, X., Gao, B., Shi, H., Slavin, M., Huang, H., Whent, M., Sheng, Y. \& L. Yu (2012). Chemical composition of 13 commercial soybean samples and their antioxidant and anti-inflammatory properties. Journal of Agricultural and Food Chemistry 6o(40):10027-10034.

https://doi.org/10.1021/jf303039a

Zhou, G., R. Cummings, Y. Li, S. Mitra, H. Wilkinson, A. Elbrecht, J. Hermes, J. Schaeffer, R. Smith \& D. Moller (1998). Nuclear receptors have distinct affinities for coactivators: characterisation by fluorescence resonance energy transfer. Molecular Endocrinology 12(10):1594-1604. https://doi.org/10.1210/mend.12.10.0176

Zhou, J., G.G. Harrigan, K.H. Berman, E.G. Webb, T.H. Klusmeyer, \& M.A. Nemeth (2011a). Stability in the composition equivalence of grain from insect-protected maize and seed from glyphosate-tolerant soybean to conventional counterparts over multiple seasons, locations, and breeding germplasms. Journal of Agricultural and Food Chemistry 59(16):8822-8828. https://doi.org/10.1021/jf2019038

Zhou, J., K.H. Berman, M.L. Breeze, M.A. Nemeth, W.S. Oliveira, D.P.V. Braga, G.U. Berger \& G.G. Harrigan (2011b). Compositional variability in conventional and glyphosate-tolerant soybean (Glycine max L.) varieties grown in different regions in Brazil. Journal of Agricultural and Food Chemistry 59:11652-11656. https://doi.org/10.1021/jf202781V

Zhu, Y., L.A. Coury, H. Long, C.T. Duda, C.B. Kissinger \& P.T. Kissinger (2000). Liquid chromatography with multichannel electrochemical detection for the determination of resveratrol in wine, grape juice, and grape seed capsules with automated solid phase extraction. Journal of Liquid Chromatography \& Related Technologies 23(10):1555-1564. https://doi.org/10.1081/JLC-100100434 
Zhu, D., N.S. Hettiarachchy, R. Horax \& P. Chen (2005). Isoflavone contents in germinated soybean seeds. Plant Foods for Human Nutrition 60:147-151. https://doi.org/10.1007/s11130005-6931-0

Zinser, C., T. Jungblut, W. Heller, H.K. Seidlitz, J.P. Schnitzler, D. Ernst \& H. Sandermann (2000). The effect of ozone in Scots pine (Pinus sylvestris L.): gene expression, biochemical changes and interactions with UV-B radiation. Plant, Cell \& Environment 23:975-982. https://doi.org/10.1046/j.1365-3040.2000.00613.x

Zitka, O., J. Sochor, O. Rop, S. Skalickova, P. Sobrova, J. Zehnalek, M. Beklova, B. Krska, V. Adam \& R. Kizek (2011). Comparison of various easy-to-use procedures for extraction of phenols from apricot fruits. Molecules 16(4):2914-36.

https://doi.org/10.3390/molecules16042914, http://www.ncbi.nlm.nih.gov/pubmed/21464799 


\section{Sammanfattning}

Under lång tid har studier av näringsmässiga och toxikologiska aspekter av vår mat fokuserat speciellt på dess innehåll av primära metaboliter. De tre senaste årtiondena har betydelsen av de sekundära metaboliterna i maten diskuterats mer och mer. En grupp av ämnen som kommit i rampljuset i de diskussionerna är fytoöstrogenerna. Detta är naturliga ämnen från växtriket som beter sig på ett sätt som liknar östrogenernas. Man har på olika sätt, på djur eller i andra testsystem, visat att de har hormonell aktivitet, antingen i form av den oförändrade molekylen eller som en metabolit bildad i kroppen. Det aktiva ämnet binder till östrogenreceptorn men med en affinitet till receptorn som är många gånger svagare än den naturliga liganden $17 \beta$ estradiol. Livsmedelskontaminanter som bildats av mikrosvampar och har östrogen aktivitet benämns inte fytoöstrogener utan mykoöstrogener. Dessa har inte beaktats $\mathrm{i}$ denna rapport.

En orsak till att fytoöstrogenerna hamnat i strålkastarljuset är behovet att sätta människors och djurs exponering för miljökontaminanter med östrogen aktivitet (till exempel metaboliter av bekämpningsmedel och bisfenol A) i ett realistiskt perspektiv. Har de ämnen som introducerats av människan östrogena egenskaper som kvalitativt eller kvantitativt skiljer sig från de som förmedlas av naturliga östrogena ämnen i vår mat?

I utvärderingen av om fytoöstrogena komponenter i vår mat kan resultera i negativa effekter eller möjligen reducera frekvensen av skadliga effekter, är det speciellt viktigt att ha en god kunskap om halten av de olika fytoöstrogenerna i vanliga livsmedel. Den här rapporten har haft i avsikt att sammanställa vår nuvarande kunskap om nivån av fytoöstrogener i färska obearbetade livsmedel. Faktorer som påverkar nivån av fytoöstrogener i färska livsmedel har granskats kritiskt och även matberedningens inflytande på halten i den mat vi konsumerar har utvärderats.

Det tycks troligt att de växter vi domesticerat och använder som livsmedel, likt andra växter, som ett instrument i sitt eget försvar mot fodersökande djur och angripande organismer har utvecklat sätt att avskräcka dem. Ett sådant sätt skulle kunna vara att påverka angriparnas signalsystem, till exempel det hormonella. Önskar man utreda denna möjlighet, krävs naturligtvis studier av interaktionen mellan det kemiska ämnet med försvarsegenskaper och de organismer som konsumerar växten, men även en kartläggning av var det kemiska ämnet förekommer och vilka halterna är. Den här rapporten avser att bidra till den senare aspekten och fokuserar på förekomsten av fytoöstrogener i mat.

Informationen om livsmedels innehåll av fytoöstrogener insamlades från vetenskaplig litteratur som identifierades vid sökningar i databasen SciFinder och i vissa fall i PubMed till och med juni 2014. Söksträngen som användes vid sökningarna innefattade termerna fytoöstrogener, de kemiska gruppnamnen (isoflavoner, lignaner, stilbener och coumestaner), namnen på de enskilda ämnena, och söktermer 
för olika livsmedel. Ytterligare referenser återfanns i referenslistorna till den litteratur som identifierades, med undantag för coumestanerna för vilka tiden var för knapp och genomföra denna extra sökning.

Identifierad litteratur granskades på data om livsmedlens innehåll av fytoöstrogener och dessa data kvalitetsgranskades. Data från dåliga studier användes inte. Då vissa publikationer presenterade data på färskviktsbasis och andra på torrviktsbasis och de olika forskargrupperna använt olika kemisk analysmetodik för att isolera och kvantifiera de olika ämnena, var det nödvändigt vid sammanställningen av data att ibland redovisa data både på färskviktsbas och också torrviktsbas, samt att separera data som erhållits med metoder som endast kan eller avsåg att analysera totalmängden av en fytoöstrogen från data erhållna med metoder som kan analysera för enskilda intakta ämnen.

Rapporten sammanfattar vår nuvarande kunskap om halten av följande fytoöstrogener i det färska livsmedlet: isoflavoner, lignaner, stilbener och coumestaner.

Isoflavoner och coumestaner analyserades med likartad metodik, men den har förändrats över tiden. De tidiga studierna fokuserade på att bestämma totalmängden av isoflavoner och coumestaner. I dessa användes extraktions- och uppreningsmetoder som hydrolyserar konjugat och kvantifierar de genererade isoflavonidaglykonerna daidzein, genistein och glycitein, samt coumestanen coumestrol. Senare studier av isoflavonerna har ofta använt metoder som avsett att analysera varje enskilt ämne som förekommer i växten/livsmedlet och dessa har analyserat för tolv olika isoflavoner (och coumestrol); de tre aglyconerna nämnda ovan, tre glukosider, tre malonylglukosider och tre acetylglukosider. Vi vet nu att det är malonylglukosiderna och glukosiderna som kraftigt överväger i växten. Baljväxter är speciellt rika på isoflavoner, men mängden varierar stort från art till art. Stora mängder har påvisats i sojaböna, klöver (både vit- och rödklöver) och alfalfa. Andra livsmedel som innehåller påtaglig mängd av isoflavoner och coumestaner är produkter med sojaböningredienser, vissa typer av bröd, energistänger och vissa bär.

Lignaner förekommer i växtvävnader som blad, stam, blommor och fröskal. De förekommer både som glykosider och aglyconer, men de har kvantifierats i aglycon form. Livsmedel rika på lignaner är cerealer och frön, vissa baljväxter, grönsaker och frukter. Haltdata finns på sex lignaner: secoisolariciresinol, lariciresinol, matairesinol, syringaresinol, pinoresinol och medioresinol. När lignanerna konsumeras metaboliseras de flesta av tarmens bakterier till däggdjurslignanerna enterodiol och enterolakton som har östrogen aktivitet.

Stilbener är bäst kända för deras förekomst i druvor och druvprodukter, men de finns även i livsmedel som öl (från humlen) och mullbär. Den kemiska analysen av processade produkter som till exempel vin har visat att de förekommer i såväl aglycon form (resveratrol), glukosid form (piceid) som dimer form (t.ex. vinefriner). Produkterna innehåller vanligen både trans- och cis-former av resveratrol och piceid. Studiet av friska druvor har visat att i dessa förekommer huvudsakligen transformerna av resveratrol och piceid, vilket antyder att cis-formerna bildas genom isomerisering vid tillverkningen/tillagningen eller när växten utsätts för stress. Den 
speciellt toxiska dimeren vinefrin produceras sannolikt i det orkestrerade försvar som startar när växten stressas.

Faktorer som påverkar halten av fytoöstrogener i den färska växten har granskats kritiskt och diskuteras i rapporten. Det framgår att flertalet av de behandlade fytoöstrogenerna är fytoalexiner. De produceras således i växten när denna stressas av abiotiska (klimatfaktorer, UV-ljus och ozon) eller biologiska (t.ex. attack av insekter eller mikrosvamp) inducerare. Mot den bakgrunden är det förståeligt att många undersökningar funnit låga halter av fytoöstrogener, sannolikt i friska växter, medan vissa andra funnit högre halter av fytoöstrogener, möjligen i stressade växter. Det är därför osannolikt att endast ett fåtal livsmedelsprov på ett adekvat sätt kan beskriva halten av fytoöstrogener $\mathrm{i}$ livsmedel. Beredningen och tillagningen av livsmedel avlägsnar eller bryter ned en viss fraktion av många fytoöstrogener, men en viss andel av termotoleranta fytoöstrogener förväntas bli kvar i livsmedlet.

Det fanns inte tillräckligt med data för att jämföra nivåerna av fytoöstrogener $i$ livsmedel producerade i Norden med nivåerna i livsmedel som importerats från andra länder till Norden. 
Nordic Council of Ministers

Nordens Hus

Ved Stranden 18

DK-1061 Copenhagen K

www.norden.org

\section{Phytoestrogens in foods on the Nordic market}

Phytoestrogens are plant-derived compounds that may bind to estrogen receptors, but with less affinity than the natural ligand estradiol. They may be biologically active as such or after metabolization in our body. To investigate the occurrence and level of phytoestrogens, scientific literature was screened for data on isoflavones, lignans, stilbenes and coumestans in raw and processed foods of plant origin. The review presents data based both on analytical methods hydrolysing glucosides and non-destructive methods.

Many phytoestrogens are phytoalexins. Their production is induced when plants are exposed to abiotic and/or biotic stress. This could explain the rather different levels reported in plants by various investigators, and indicates that many samples are required to describe the levels generally occurring in foodstuffs. The influence of food processing was also considered. 